\title{
Current status and challenges of fabricating thin film composite forward osmosis membrane: A comprehensive roadmap
}

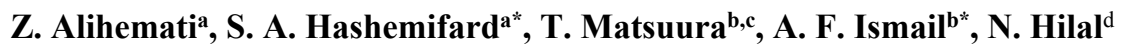 \\ ${ }^{a}$ Sustainable Membrane Technology Research Group (SMTRG), Faculty of Petroleum, Gas and Petrochemical \\ Engineering(FPGPE), Persian Gulf University (PGU), P.O. Box 75169-13798, Bushehr, Iran \\ ${ }^{b}$ Advanced Membrane Technology Research Centre (AMTEC), Universiti Teknologi Malaysia, 81310 UTM Skudai, \\ Johor Darul Ta'zim, Malaysia \\ ${ }^{c}$ Department of Chemical and Biological Engineering, University of Ottawa, 161 Louis Pasteur St., Ottawa, ON, \\ CanadaK1N 6 N5 \\ ${ }^{d}$ Centre for Water Advanced Technologies and Environmental Research (CWATER), Swansea University College of \\ Engineering, Swansea, United Kingdom
}

\begin{abstract}
Recently, forward osmosis (FO) has attracted a great deal of attention in desalination and wastewater treatment. Nevertheless, there are several critical challenges such as the need for new advances in designing membranes that must be met to enhance the water flux in FO processes, control the reverse salt flux, concentration polarization and fouling. Therefore, designing a suitable membrane with a high-water flux, low reverse salt flux, low fouling, and controlled concentration polarization seems to be essential. Thin film composite (TFC) membranes are the most widely used membranes in the FO field. Extensive research has been performed to fabricate and design high performance TFC membranes which can be exclusively used in FO processes. This paper aims to review three types of TFC membranes i.e. TFC's with polyamide active layer (TFC-A), thin film nanocomposites (TFC-N) and double-skinned TFC membranes (TFC-D) in flat sheet and hollow fiber configuration. Finally, an attempt is made to generate a general performance curve based on the water flux and reverse salt flux of these three TFC FO types and the future direction of the R and D on the FO membrane are discussed.
\end{abstract}

Key words: Forward osmosis; Water treatment; Desalination; Thin film composite membrane; Thin film nanocomposite membrane 


\title{
2 Table of Contents
}

\author{
3 1. Introduction \\ 4 2. Thin film composite membrane (TFC) \\ $5 \quad 2.1$. Synthesizing porous substrates \\ 6 2.2. Synthesizing active layers \\ 7 2.3. Performance of TFC-A flat sheet membranes \\ 8 2.3.1. Effect of MPD and TMC concentration on active layer \\ 9 2.3.2. PSF as substrate modified with polydopamine \\ 2.3.3. Polyacrylonitrile (PAN) as substrate \\ 2.3.4. Nylon 6.6 as substrate \\ 2.3.5. Polyethylene as a substrate \\ 2.4. Performance of TFC-A hollow fiber membranes \\ 2.4.1. PES as substrate \\ 2.4.2. Polyphenylene sulfone as substrate \\ 2.4.3. Matrimid as substrate \\ 2.4.4. PAN as substrate \\ 2.4.5. Polyketone as substrate \\ 2.5. Issues and Implications of TFC-A membranes \\ 2.6. Performance of TFC-N membranes \\ 2.6.1 TFC-N with modified substrate \\ 2.6.2. TFC-N with modified active layer \\ 2.6.3.TFC-N with modified both substrate and active layer concurrently \\ 2.8. Performance of TFC-D membranes \\ 2.8.1. Inner active layer RO polyamide and outer skin layer NF polyethyleneimine \\ 2.8.2 Both skin layers from polyamide \\ 3. Commercial FO membranes \\ 3.1. HTI membrane \\ 3.2. Aquaporin $\mathrm{A} / \mathrm{S}$ membrane \\ 33 \\ 4. Proposing roadmap, concluding remarks and future directions \\ 5.Refrences
}

34 


\section{Introduction}

Today, supplying adequate freshwater is crucial for human life and survival of industries. Considering the progressive growth of population and the limitation of the world freshwater reserves, rapid and economical desalination of saline/brackish water and treatment of wastewater have become increasingly important to provide clean water for different purposes [1-4]. Currently, reverse osmosis (RO) has a wide range of applications in water treatment processes due to its superiority over the other conventional methods [5-8]. Nevertheless, the energy consumption of RO is still high despite the remarkable progresses and many efforts made during the past several decades to reduce it, due primarily to the intrinsic thermodynamic constraints of the membrane desalination process [6]. Hence, the minimum amount of energy required for complete separation is at least equal to or greater than the free enthalpy of mixing [9]. Furthermore, extensive fouling and high retentate concentration are considered as the serious challenges of RO plants [10]. Recently, a novel membrane process known as forward osmosis (FO) has been proposed with the aim of saline water desalination as well as wastewater treatment [11-17]. FO applies osmotic pressure differences as a driving force to induce a net flow of water across the membrane from a feed solution (low osmotic pressure) to a draw solution (high osmotic pressure)[18-21]. Although FO process has several advantages over the conventional methods, this method also suffers from various challenges and disadvantages at present. The advantages of FO include its low energy consumption to transfer water through a semi-permeable membrane and relatively low fouling tendency [13, 22-25]. However, an additional process is required to prepare the final product, i.e. pure water, since the product cannot be consumed directly as fresh water [26-29]. The absence of properly designed FO membrane and inexpensive draw solution are considered as the other FO challenges [26, 29-36]. There are two types of membranes for FO: a) membranes originally made for RO and nanofiltration (NF); b) membranes specifically fabricated for FO [37]. An ideal FO membrane should consist of an active layer of high water permeability and low reverse solute diffusion [38] (representing the amount of salt that migrates from the draw solution towards the feed [10]) and a high flux substrate. Also, the membrane is expected to be chemically and mechanically stable showing less tendency to fouling and concentration polarization [39, 40]. Among the polymer materials that are used for synthesizing FO membranes, recent studies have focused on materials such as cellulose derivatives, polyamide (PA), polyelectrolyte, and polybenzimidazole (PBI). Cellulose derivatives such as cellulose triacetate (CTA) membranes are fabricated as flat sheet and hollow fibers via the phase inversion process followed by heat treatment [39]. Basically, they are integrally skinned asymmetric membranes. In particular, CTA membranes have been already commercialized by HTI Co. [41]. The thickness of these CTA membranes is less than the standard RO membranes [42]. The embedded polyester mesh is applied as a mechanical support for the CTA HTI membranes. Although, they exhibit higher water flux and salt rejection compared to the commercial RO membranes in FO process, the water fluxes are still far lower than the desirable values [43]. Moreover, the membranes made of cellulose derivatives suffer from the limited resistance to high $\mathrm{pH}$, high temperature[44], compaction and biofouling [43]. In addition, Aquaporin-Incorporated Biomimetic Membranes are the high-performance FO 
membranes, which are made by incorporating Aquaporins into biomimetic membranes [45]. The poor mechanical stability and the complex fabrication process of this type of membranes are its main drawback [46]. In this respect, thin film composite (TFC) membranes seem to be more practical and superior to the old-style integrally skinned asymmetric membranes. They can function under a wider $\mathrm{pH}$ variation, resist high temperature media and are stable to high pressure and biodegradation problems [47]. A TFC membrane consists of at least two distinct layers, a top active layer and a porous substrate, each layer plays its special role [48]. In contrast to the integrally skinned asymmetric membranes, in which both active layer and the substrate layer simultaneously are made from the same material, in TFC membranes the active layer and the substrate layer can be optimized individually by using different materials and methods [49]. Thus, TFC membranes usually exhibit higher water flux and salt rejection relative to integrally skinned membranes in RO. Similarly, TFC membranes may exhibit better performances in FO than that of integrally skinned asymmetric membranes [50]. Despite these advantages, TFC membranes still suffer from the internal concentration polarization (ICP) in FO which severely diminishes the flux [39, 51-53].

Review articles on FO process have been published on topics such as basic principle of FO, challenges and application $[2,43,46,48,54]$; hybrid FO process $[21,37,55-57]$; draw solutes $[6$, 58, 59]; membrane fouling [60-63] and membrane material and fabrication [39, 64-68]. However, a review presenting detailed information on the fabrication and development of TFC FO membranes as the most widely used membrane in this area is currently lacking. This paper presents an overview on the recent advances in TFC FO membrane and the important factors such as the method and material of synthesizing active layer and substrate, which affect the TFC performance. The methods that are used for improving water flux $\left(\mathrm{J}_{\mathrm{v}}\right)$ and reducing the ratio of reverse salt flux $\left(\mathrm{J}_{\mathrm{s}}\right)$ (i.e. reverse solute diffusion from draw solution to feed solution) to water flux (specific reverse salt flux $\left.\left(\mathrm{J}_{\mathrm{S}} / \mathrm{J}_{\mathrm{v}}\right)\right)$ in TFC FO membrane are described. Also, in the conclusion section, a comprehensive roadmap based on the reviewed papers is proposed and discussions are made on the future direction for the FO membrane research and development. In the authors' view, this review can offer a useful guideline for the fabrication of more ideal TFC FO membranes and the promotion of FO process.

The classification of TFC FO membranes in this review is shown in Fig. 1. Three types of TFC membranes are presented: a) TFC with a polyamide active layer (TFC-A), b) Thin film nanocomposite (TFC-N) c) Double-skinned TFC (TFC-D). "TFC-A" includes TFC FO membranes, either modified or unmodified, with a polyamide active layer without nanomaterials, "TFC-N" includes all TFC FO membranes that contain nanomaterials and "TFC-D" includes TFC FO membranes with two active layers. 


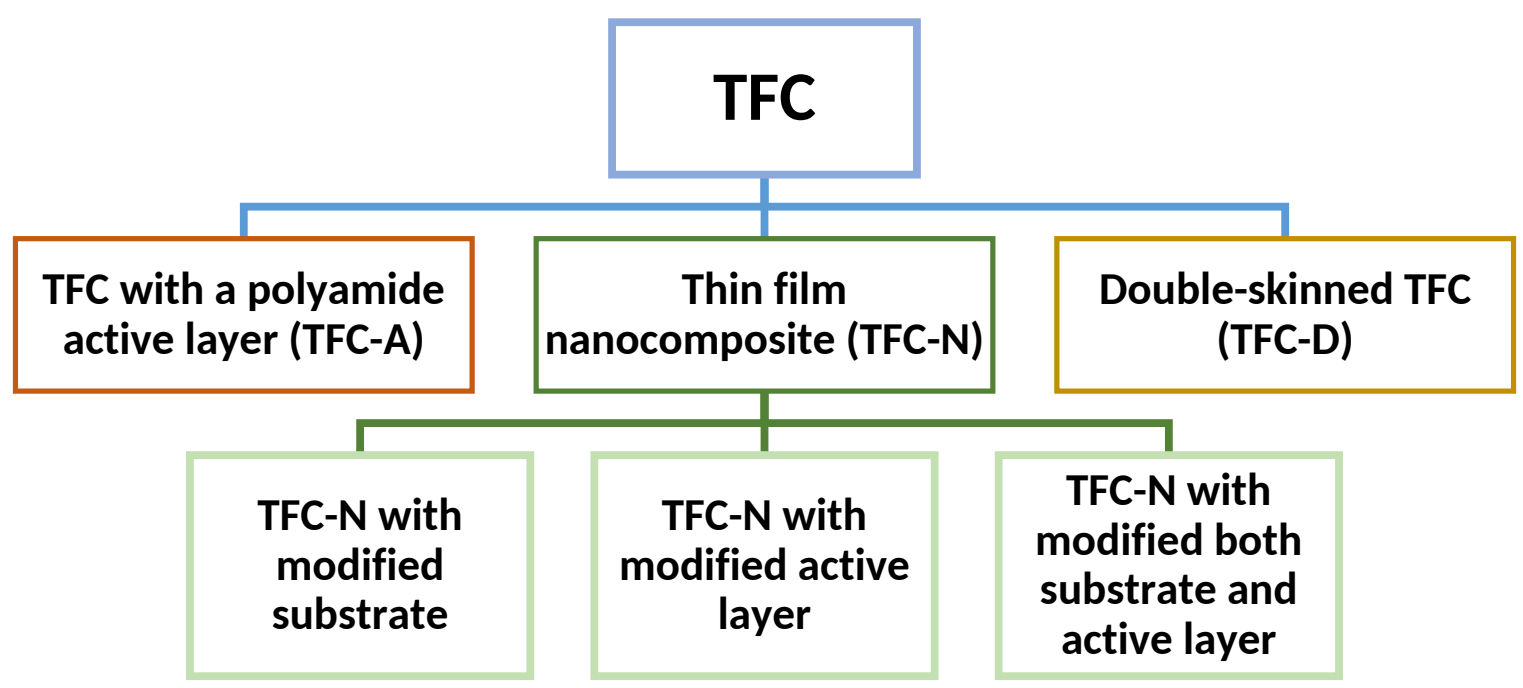

1

Fig 1: The classification of TFC membranes used in FO process

The TFC-A type is further divided into two groups according to their configuration: a) flat sheet and b) hollow fiber. To the best of the authors' knowledge, TFC-N membranes for FO application are mostly fabricated in the flat sheet configuration. Although the TFC-D type has been fabricated in the two different configurations, the number of TFC-D membranes are small. Hence, TFC-N and TFC-D membranes are discussed without splitting into two different configurations. It should be noted that many effects of membrane preparation on membrane performance are common for both flat sheet and hollow fiber configuration.

\section{Thin film composite membrane (TFC)}

Since 1980, TFC membranes have been utilized for water desalination by RO. In spite of the advances and improvements of membranes over the past years, the principles of their design have not been changed fundamentally. Polyamide TFC membranes are currently the most popular membranes for desalination in the commercial market [49].

\subsection{Synthesizing porous substrate}

As mentioned earlier, the TFC membrane consists of two distinct layers, the thin selective layer and the porous substrate. The latter layer is produced typically from polysulfone (PSF) and polyethersulfone (PES) by phase inversion method [39]. PSF and PES are the preferred material thanks to their good chemical and suitable mechanical resistance [66]. Occasionally, in the stage of preparing the membrane substrate, the polymer solution is cast on a polyester backing material [69]. Both woven and nonwoven polyester can be used [70]. The backing material does not pose 
any resistance against the flow of water and is only used to enhance the mechanical strength of the membrane [69]. A three-layer membrane is schematically shown in Fig. 2.

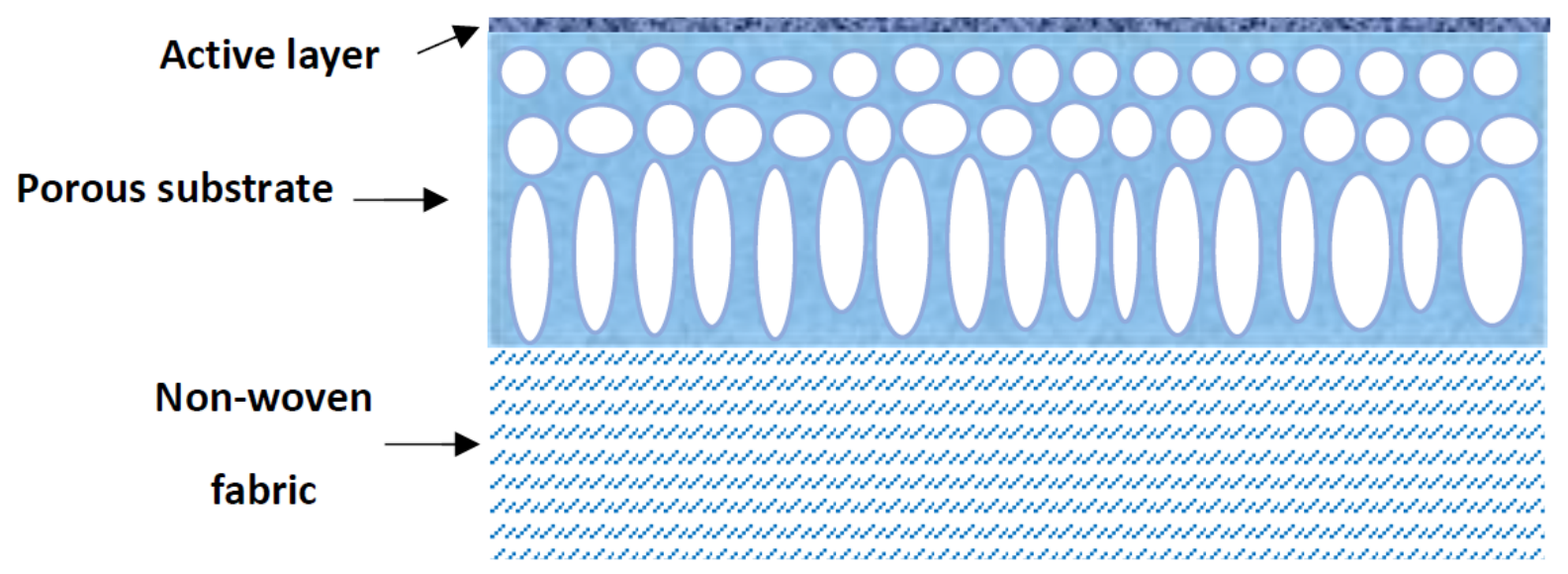

Fig 2: Diagram of a three-layer TFC membrane

Although the phase inversion method is one of the most prevalent methods for synthesizing the substrate of FO membranes, it has been encouraged recently to use the electro-spinning method to produce nanofiber polymeric substrate instead of the phase inversion method because it yields more satisfactory results in terms of the FO Flux [39, 71]. Electro-spinning applies a high electric field to make nanofibers from a dope solution [72]. In this process, both the curvature and tortuosity of the substrate are diminished while the porosity is enhanced $[39,71,73]$. Accordingly, the structural parameter $(S)$ of the produced membrane, defined as (thickness $\times$ tortuosity/porosity) [43] diminishes compared to the conventional membranes. This parameter is defined to evaluate the degree of internal concentration polarization. Nevertheless, the mechanical strength of the nanofiber layer is lower than that of the conventional substrates [39, 71]. Fig. 3 demonstrates the schematics of fabricating flat sheet substrate for FO process applying phase inversion and electro17 spinning methods. 


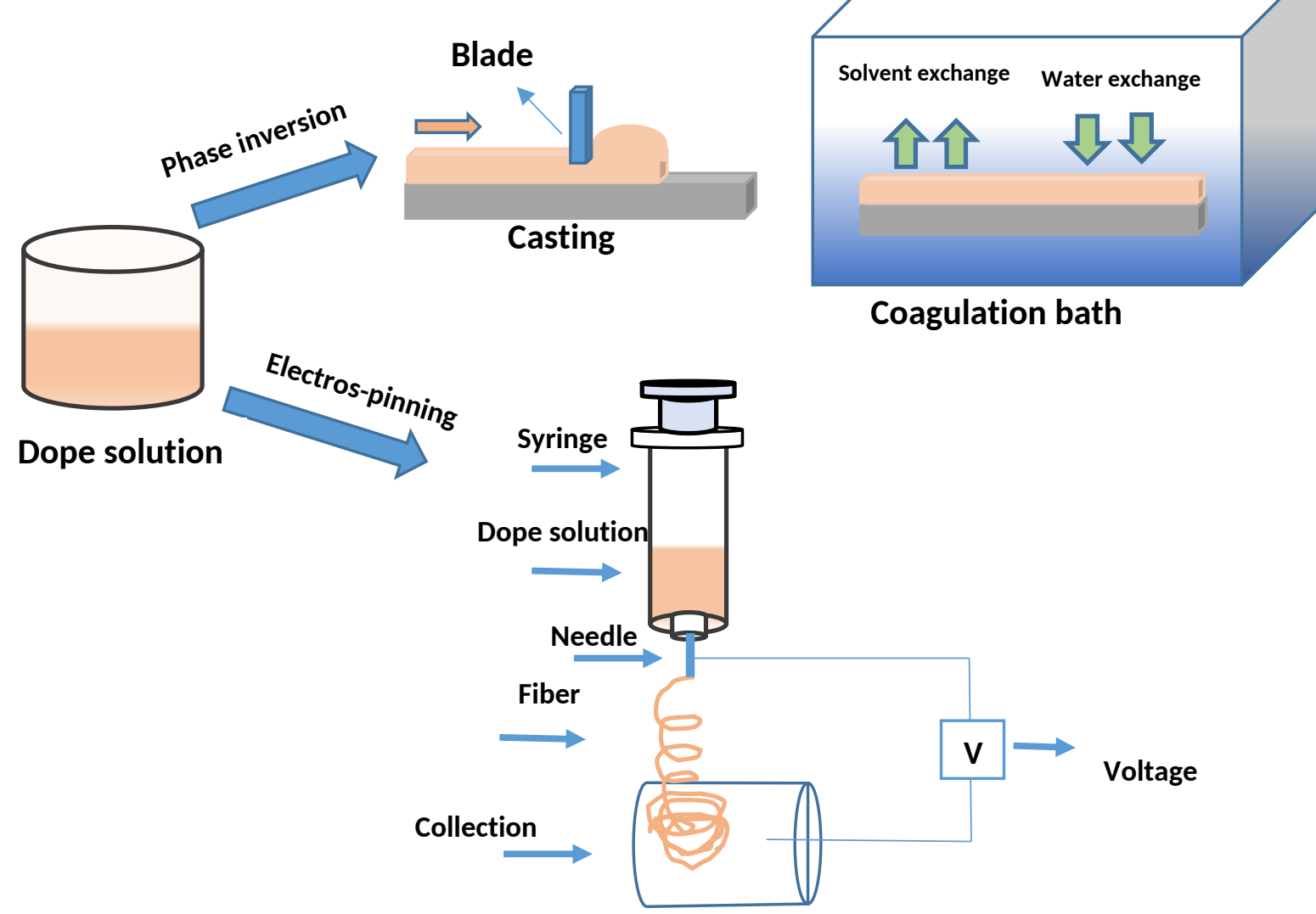

2 Fig 3: Flat sheet substrate fabricating techniques: (a) Phase inversion process (b) Electro-spinning process

\subsection{Synthesizing active layers}

5 Polyamide (PA) active layer is synthesized by the interfacial polymerization method using two 6 monomers, namely m-Phenylenediamine (MPD) in aqueous phase and Trimesoyl Chloride (TMC) 7 in organic phase [74, 75]. Fig. 4 demonstrates polyamide formation by the condensation reaction 8 of the two monomers, which takes place at the interface between the aqueous and organic phase.

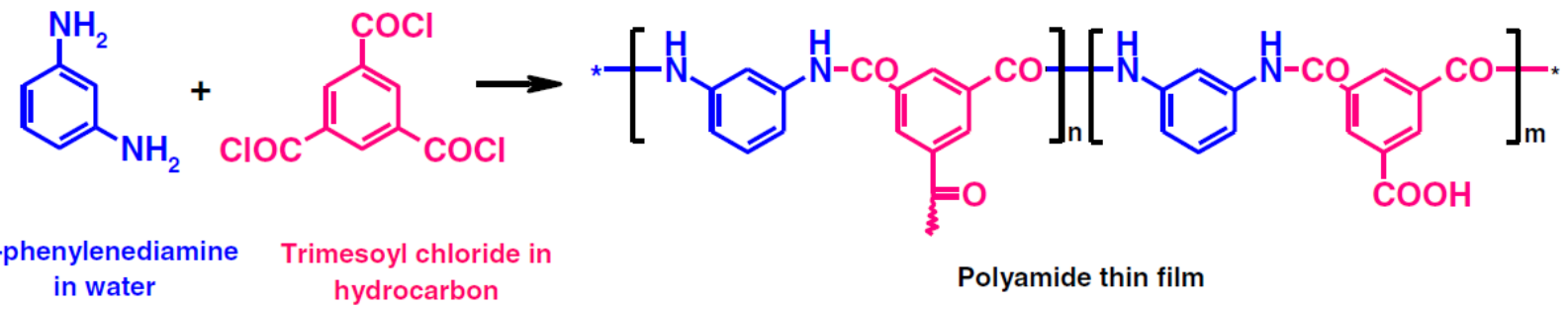

Fig 4: Interfacial polymerization reaction between MPD in water and TMC in n-hexane [74]

11 Other monomers can also be used to synthesize polyamide. Lau et al. [74] reviewed the common 12 monomers used in the synthesis of TFC membranes. They include amine monomer, such as 
Piperazine (PIP) and P-phenylene diamine (PPD) and Triethanolamine (TEOA) as well as Acyl chloride monomer, such as Isophthaloyl chloride(IPC), 5-isocyanato-isophthaloyl chloride (ICIC) and 5-chloroformyloxy-isophthaloylchloride (CFIC). Similarly, Li et al. [39] reviewed the monomers used in the synthesis of an active layer of TFC membranes and compared the properties of the synthesized active layer. In a wide range of monomers used in IP reaction to prepare the PA layer, MPD and TMC are the two most popular monomers [44]. Both MPD and TMC contain a benzene ring. Therefore, membranes fabricated by applying this chemistry are identified as fully aromatic PA membranes [76]. Indeed, aromatic cross-linked PA layer with high water flux and [77] high $\mathrm{NaCl}$ rejection[76] has mostly been provided by MPD and TMC monomers.

\subsection{Performance of TFC-A Flat sheet membranes}

To synthesize a high-performance TFC-A membrane, the properties of the substrate and the active layer of the membrane should be optimized [39]. The type of the polymer and its concentration, selecting a suitable solvent for preparing the casting solution, precipitation medium, and the use of additives are among the most effective factors in preparing the substrate [10,78-81] by phase inversion method. Various factors are involved in the final structure and performance of TFC membranes. The key parameters for the formation of the active layer by interfacial in-situ polymerization include the concentration of monomers, ratio of the monomers, type of solvent, time period of the interfacial polymerization reaction, and additives in the aqueous or organic solution [82-84].

\subsubsection{Effect of MPD and TMC concentrations on active layer}

Wei et al. [84] investigated the effect of the parameters affecting the active layer performance of a polyamide TFC-A membrane with PSF substrate. In particular, they examined the effect of MPD and TMC concentrations on the separation properties of the fabricated membranes. They found out that an increase in MPD concentration at a constant TMC concentration decreases the permeability and increases the salt rejection. It was most likely, due to the formation of denser active layer via higher degree of cross-linking. On the other hand, when TMC concentration is increased at a constant MPD concentration, the water permeability increases while salt rejection decreases, due to the increase in acyl chloride content and decrease in the extent of the crosslinking. They also stated that membranes with less salt rejection have a greater reverse solute diffusion and thus higher ICP. The performance of FO membrane is controlled by both permeability and salt rejection. When the main limiting factor of water flux is the friction loss, and also when the concentration of the draw solution is low, permeability has a greater effect than the salt rejection on water flux. Conversely, when the ICP is severe, salt rejection plays a more important role to govern the water flux. In the latter case, it is recommended to make the membrane active layer denser. 
Fig. 5 indicates the effect of MPD concentration on the water flux and specific reverse salt flux in two different orientations, FO (AL-FS) or PRO (AL-DS) orientation, and using two different draw solution concentrations. Specific reverse salt flux represents the selectivity which is unfavorable in different aspects: a) increase in the reverse solute diffusion causes aggravation of membrane fouling and increases accumulation of solutes in the feed solution side; $b$ ) it also increases the cost of replacing the draw solution. Water flux dependence on TMC concentration in a FO process is as complex as the effect of MPD concentration on the water flux. Furthermore, the optimization in FO is contingent upon the details of the applied conditions including the draw solution concentration and the membrane orientation. An optimal membrane in a particular condition does not necessarily yield the same results under new conditions. In the next sections the effect of the factors such as method and material of synthesizing substrates, that affect the TFC-A performance are discussed for polymers that are often used for substrate preparation.
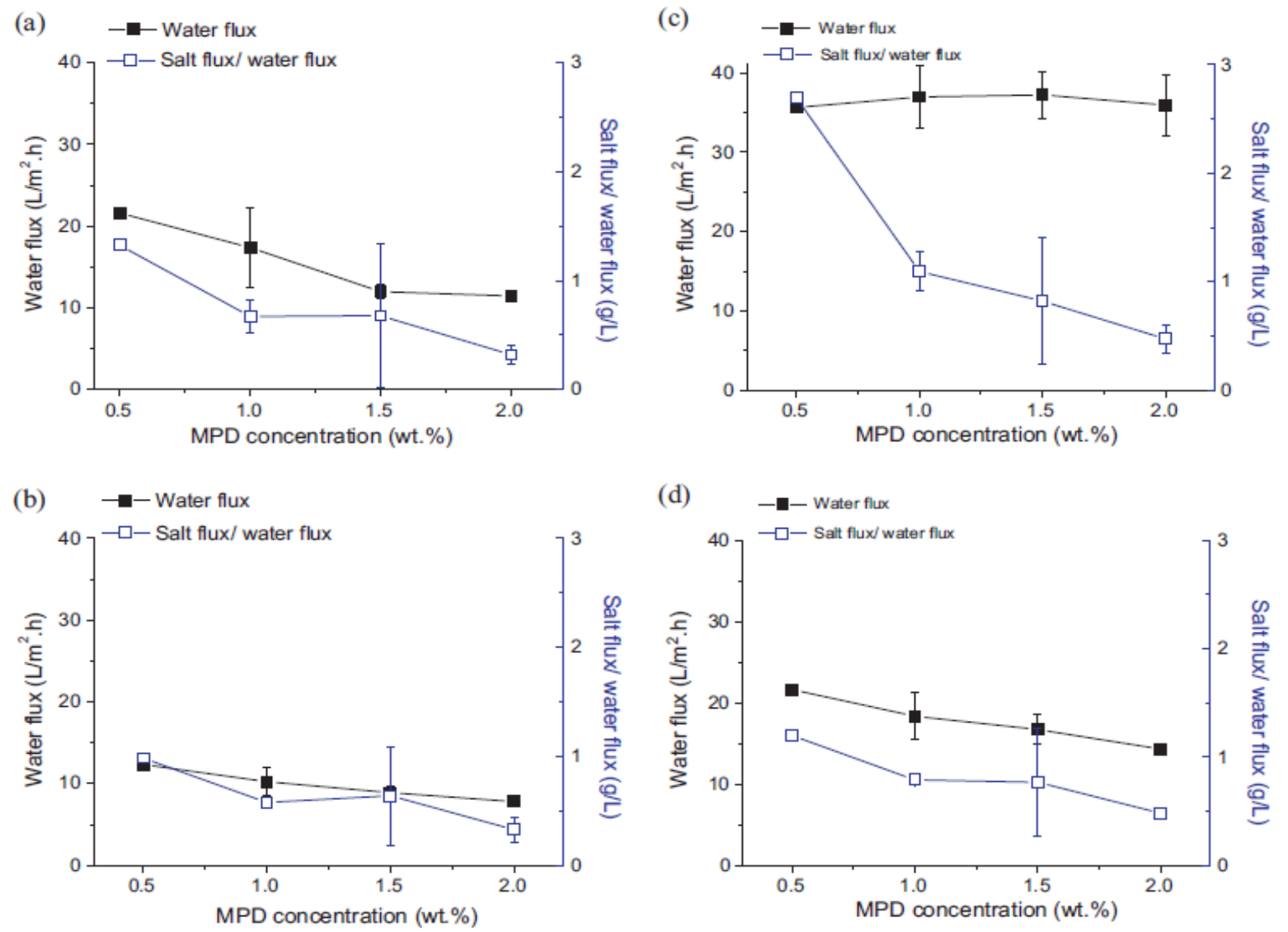

Fig 5: Performance of FO of TFC-A membranes fabricated with various MPD concentrations at fixed TMC concentration of $0.5 \mathrm{wt} . / \mathrm{v} \%$. (a) AL-DS and (b) AL-FS orientations for a $0.5 \mathrm{M} \mathrm{NaCl}$ draw solution; (c) AL-DS and (d) AL-FS orientations for a $2.0 \mathrm{M} \mathrm{NaCl}$ draw solution. The feed solutions contained $10 \mathrm{mM} \mathrm{NaCl}$ [84]. 


\subsubsection{PSF as substrate modified with polydopamine}

2 Han et al. [85] used a natural polymer called polydopamine (PDA) to improve the separation 3 performance of FO composite membranes. Polydopamine, as a hydrophilic polymer with adhesive

4 properties, has been used to enhance antifouling property in UF, NF, and RO by enhancing 5 hydrophilicity of the active layer and substrate of the membrane. The presence of a hydrophilic 6 substrate is also essential in FO membrane to mitigate the effect of ICP and to enhance the water 7 flux. In their work, PDA was coated on the top surface of a PSF substrate via self-polymerization 8 before the active layer was synthesized by in-situ polymerization. Experimental results have shown

9 the increase in water flux and salt rejection as well as the reduction of ICP. In addition to enhancing 10 the hydrophilicity of the internal walls of the substrate, PDA plays another positive role in 11 developing a polyamide active layer by creating a smooth hydrophilic surface with small pores. 12 Moreover, PDA interacts with TMC monomers and makes the active layer defect-free. It also 13 results in enhanced stability between the active layer and substrate.

\subsubsection{Polyacrylonitrile (PAN) as substrate}

Klaysom et al. [83] synthesized a TFC membrane with polyacrylonitrile (PAN) substrate. PAN is more hydrophilic than PSF and PES. In addition, PAN has high thermal and chemical resistance as well as resistance against the normal solvents but the nitrile group is sensitive to alkaline environment [66]. The main aim of Klaysom et al. work was to determine the essential parameters 20 that affect the fabrication of TFC-A membranes especially in the interfacial polymerization stage. 21 The parameters considered were the type of the additives and the solvent evaporation period. As 22 shown in Fig. 6, the substrate consists of three parts, namely the dense skin layer, the upper part 23 of sublayer with smaller macrovoids and the lower part of the substrate with larger macrovoids. 24 They have reported that the structural parameter of the PAN substrate was lower than that of the 25 commercial HTI membranes [83]. 
a

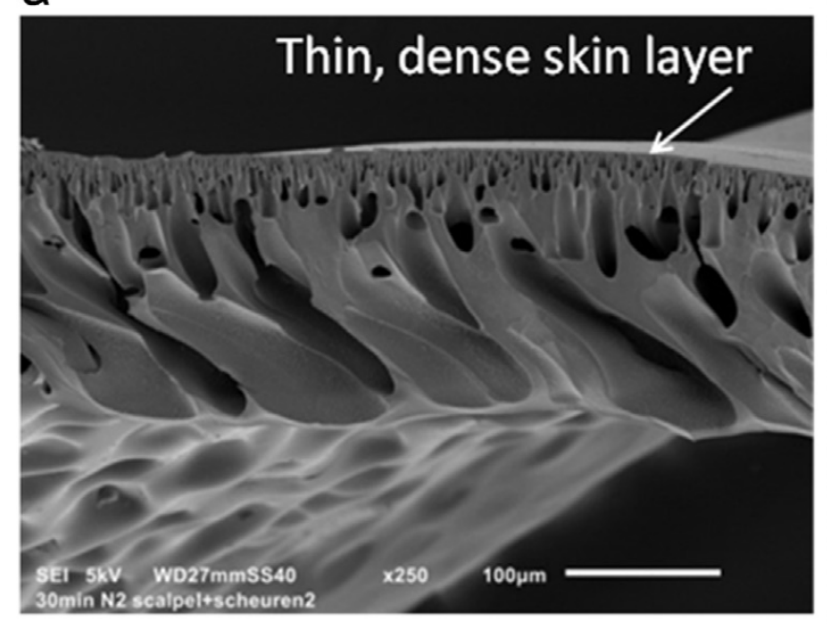

\section{C}

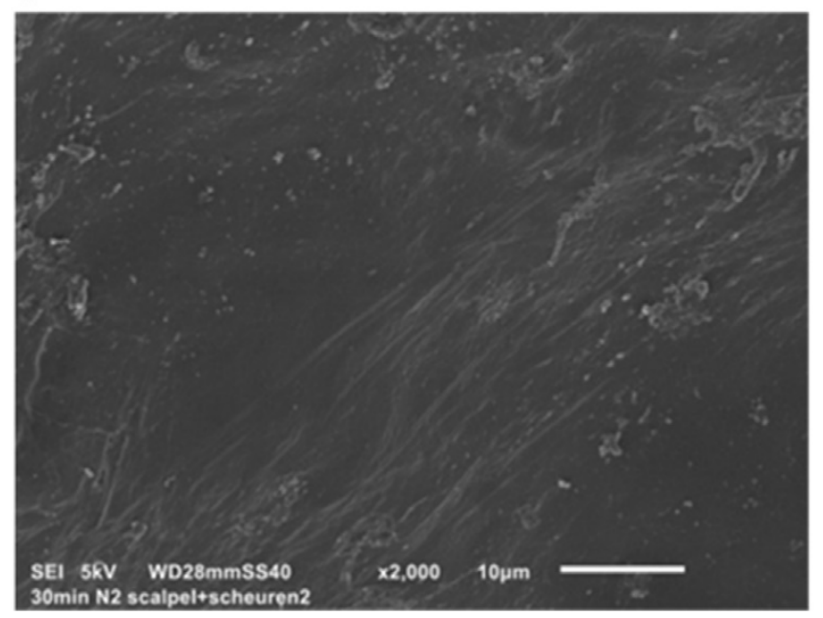

b

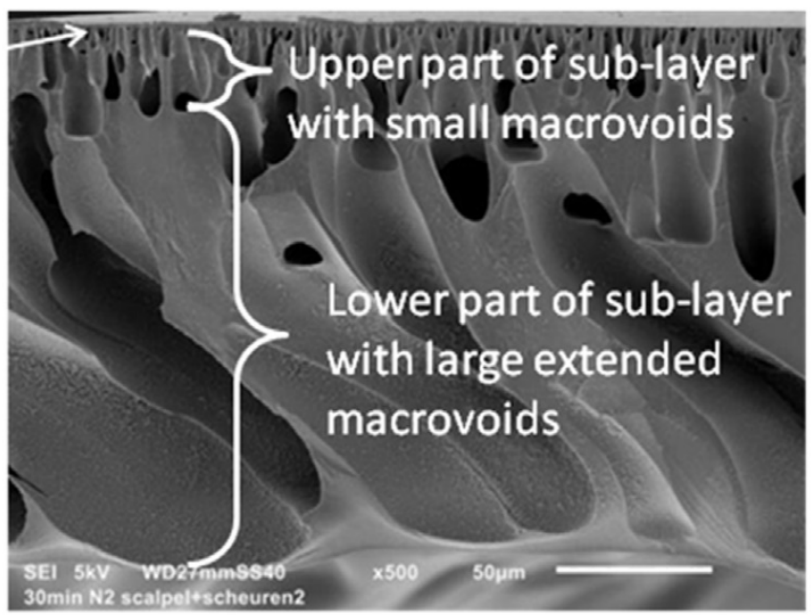

d

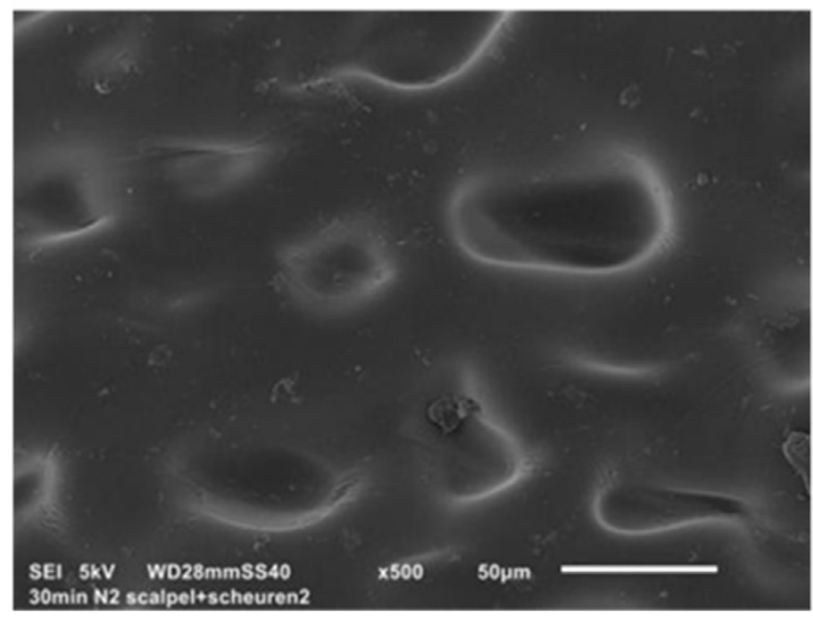

Fig 6: SEM images of (a and b) cross-section, (c) top surface, and (d) bottom surface of the PAN support layer [83]

After fabrication of the substrate, the active layer was synthesized via interfacial polymerization technique utilizing MPD and TMC as monomers. First, the substrate was immersed in an MPD aqueous solution for $30 \mathrm{~min}$. After clearing the extra amine solution from the substrate surface by a clean cloth, the substrate surface was dried for 1-3 min in a fume hood at ambient temperature. Then, the substrate surface was contacted with the TMC organic solution for 20-80 s, followed by washing with n-hexane to remove the unreacted solution. The membranes were further dried at the ambient condition for $1 \mathrm{~min}$ before being stored in deionized water at room temperature prior to use. By increasing the drying time after soaking in the amine solution, the salt rejection increased while the permeability gradually diminished. In addition, the surface roughness of the membrane displayed a decreasing trend [83]. It is well known that the polycondensation reaction takes place in the organic phase. Therefore, the stability of the polyamide layer depends on the closeness of the two phases interface to the surface of the substrate. If the substrate surface is cleared and cleaned well, MPD solution lies inside the substrate and exactly in the vicinity of the surface. This 
in turn causes the formation of an absolutely stable and strong active layer. On the other hand, if the substrate surface is not sufficiently cleaned and dried to make it free from the amine solution, the contact area of the two phases might be detached from the top substrate surface causing the formation of a flimsy and brittle polyamide layer at a distance off the substrate surface [86]. The reaction time also affects the surface morphology and permeability. By lengthening the reaction time, due to the increase in the thickness of the formed active layer, the permeability decreases. The effect of reaction time on permeability is more pronounced between 20 and $40 \mathrm{~s}$ [83], since as the time lengthens, the active layer formation rate decreases while the active layer thickness remains constant [87]. Therefore, the effect of reaction time, especially after $40 \mathrm{~s}$ on the surface morphology, is higher than its effect on the permeability [83]. Khorshidi et al. [88] also studied the effect of reaction time in a range of 15 to $60 \mathrm{~s}$, and observed that the increase in reaction time slightly decreased the permeability. Fig. 7 shows the formation of the active layer on the PAN substrate as a function of drying and reaction time. As for the effect of the substrate pore size, when the pore is small, MPD transfer occurs by diffusion and simple convection whereby a crosslinked thin film with a nodular structure is developed. When the pore size is large, MPD transfers to the organic phase much faster due to the Marangoni effect caused by the surface tension gradient between the two phases. This results in the rapid migration of MPD monomers. This rapid migration tends to push the initially formed nascent outward and twist the formed film, thus ridge and valley structure is produced [83].
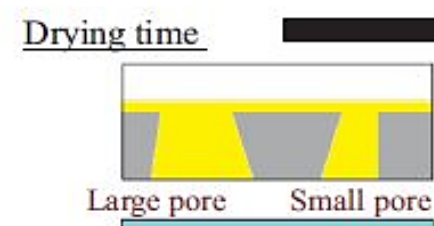

Small pore
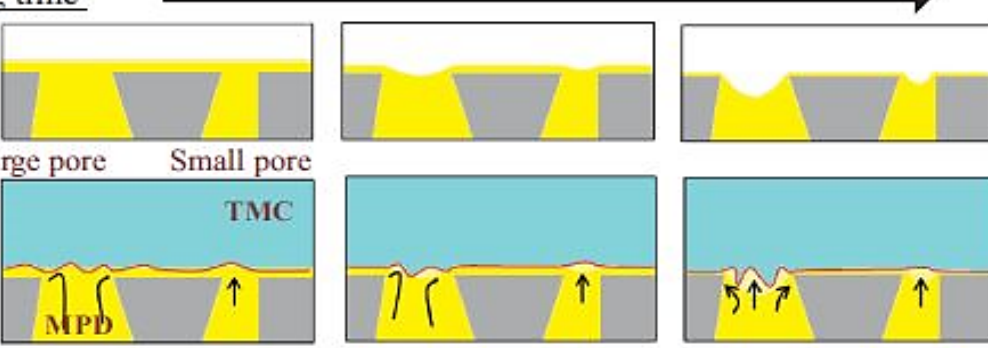

Smoother surface Reaction time
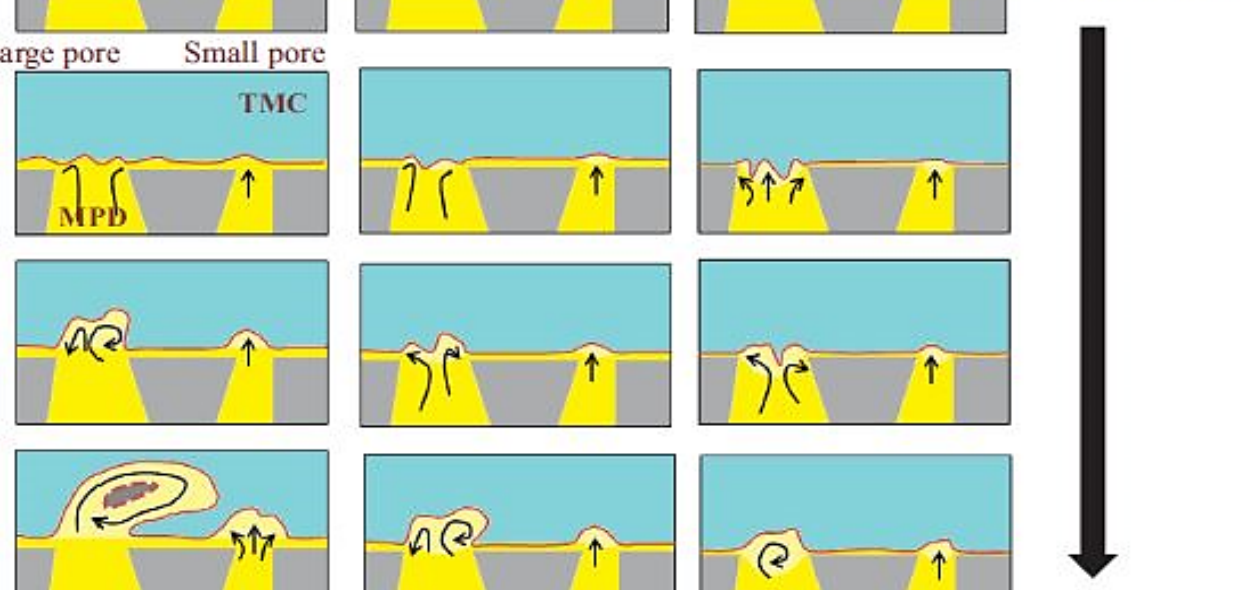

Rougher surface
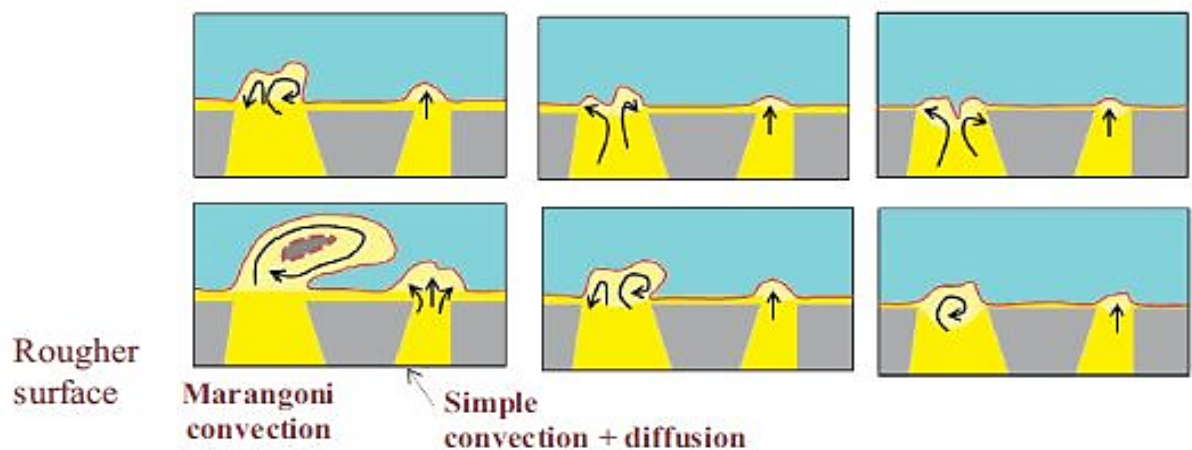

convection + diffusion

Fig 7: Scheme showing polyamide thin film formation mechanism [83] 


\subsubsection{Nylon 6.6 as substrate}

Huang et al. [89] also investigated the effect of the substrate pore size on the properties of the active layer including morphology, extent of crosslinking, mechanical integrity, selectivity, and permeability. In their research, four types of microfiltration substrates made of Nylon 6.6 with different pore sizes were provided by $3 \mathrm{M}$ Purification Inc. Nylon 6,6 is a conventional polymer for plastic and textile industry. It has a semi-crystalline structure, i.e. posing good thermal, mechanical and chemical properties. Additionally, it is more hydrophilic compared to the common polymers such as PSF. However it has less swelling propensity than other conventional hydrophilic polymers, such as cellulose acetate (CA) [90]. The general structures, as well as physiochemical properties of the substrates, were identical. In a range of pore sizes 0.025 to $0.45 \mu \mathrm{m}$, they concluded that the substrate pore size had no effect on the thickness of the active layer. However, with an increase in the pore size, the degree of crosslinking diminished, leading to the decrease in salt rejection. Additionally, they found out that the membrane mechanical strength was higher for small pores. Considering all these factors, they concluded that the substrate pore size of $0.2 \mu \mathrm{m}$ resulted in the optimum TFC membrane. Although membranes with fewer pores have a greater extent of crosslinking and higher selectivity, the balance between selectivity and permeability is important to achieve better performance. Membranes with smaller pores show lower permeabilities but higher structural parameters. Further, membranes with the pore size of $0.45 \mu \mathrm{m}$ had a lower water flux compared to the other membranes, which was due to an increase in reverse salt flux, hence increase in ICP, and reduction of driving force. An important conclusion that can be drawn, is that to synthesize high-performance membranes, the thickness, tortuosity and porosity should be controlled to minimize the structural parameter. Also, the pore size of the substrate and its effect on the structural parameter along with the performance of the membrane active layer should be taken into account [89]. Contrary to Huang et al. [89], Singh et al. [91] reported that the pore size of the PSF substrate has an effect on the active layer thickness. It is probably due to the higher hydrophobicity of PSF than Nylon 6,6, which caused the higher resistance for MPD diffusion in the smaller pores.

\subsubsection{Polyethylene as substrate modified with polydopamine}

Kown et al. [92] fabricated a high performance and outstanding durable membrane with polydopamine-modified polyethylene (DPE) substrate having an active layer formed by a unconventional interfacial polymerization method. They used an aromatic solvent-based i.e. toluene instead of an aliphatic organic solvent i.e. n-hexane, with the same monomer (TMC) in their method. The polydopamine coating uniformly hydrophilized the hydrophobic polyethylene (PE) surface, yielding a long-term stable operation. The highly porous and thin substrate structure was protected after modification of the substrate with PDA. In addition, the use of the toluenebased IP process allowed for the formation of a highly selective polyamide active layer on the hydrophilic DPE substrate, which was superior to the conventional IP method. Indeed, toluene as an aromatic hydrocarbon solvent has lower interfacial tension with water and greater MPD 
1 solubility than $\mathrm{n}$-hexane as an aliphatic hydrocarbon, which can accelerate MPD diffusion and

2 promote the IP reaction. Thus, the prepared TFC membrane with this method presented much 3 higher FO performance. Table 1 summarizes the latest studies published in the literature. The table 4 includes water flux and specific reverse salt flux of flat sheet TFC membranes with a polyamide 5 active layer. Experimental results in the two different modes (AL-FS(FO)) and AL-DS(PRO) are 6 separately reported. 
Table 1: Summary of the studies on flat sheet TFC-A FO membranes

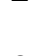

3

4

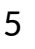

6

PES

sulphonated copolymer polyethersulfone and polyphenylsulfone support Performance

\begin{tabular}{lll}
\hline support & Performance & Experimental condition
\end{tabular}

(PES-co-sPPSU 11)

\begin{tabular}{cccc}
\multicolumn{2}{c}{ AL-FS(FO) } & \multicolumn{2}{c}{ AL-DS(PRO) } \\
\hline $\mathrm{J}_{\mathrm{v}}$ & $\mathrm{J}_{\mathrm{s}} / \mathrm{J}_{\mathrm{v}}$ & $\mathrm{J}_{\mathrm{v}}$ & $\mathrm{J}_{\mathrm{s}} / \mathrm{J}_{\mathrm{v}}$ \\
$(\mathrm{LMH})$ & $(\mathrm{g} / \mathrm{L})$ & $(\mathrm{LMH})$ & $(\mathrm{g} / \mathrm{L})$
\end{tabular}

$\begin{array}{cr}\text { Feed } & \begin{array}{r}\text { Draw } \\ \text { solution }\end{array} \\ \text { solution }\end{array}$

cross-flow velocity

Intrinsic

properties

ref

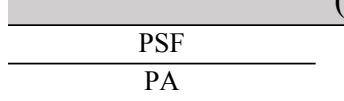

12

0.408

$20.5 \quad 0.287$

$0.5 \quad \mathrm{~m}$

$500 \mathrm{ml} / \mathrm{min}$

$\mathrm{A}=1.78 \mathrm{LMH} / \mathrm{bar}$

$\mathrm{B}=9.4 \times 10^{-8} \mathrm{~m} / \mathrm{s}$

$\mathrm{S}=0.67 \mathrm{~mm}$

$\mathrm{R}=93.4$

$=0.73 \mathrm{LMH} / \mathrm{bar}$

$\mathrm{B}=0.25 \mathrm{LMH}$

$\mathrm{S}=3.24 \times 10^{-4} \mathrm{~m}$

9

$$
\mathrm{R}=91
$$

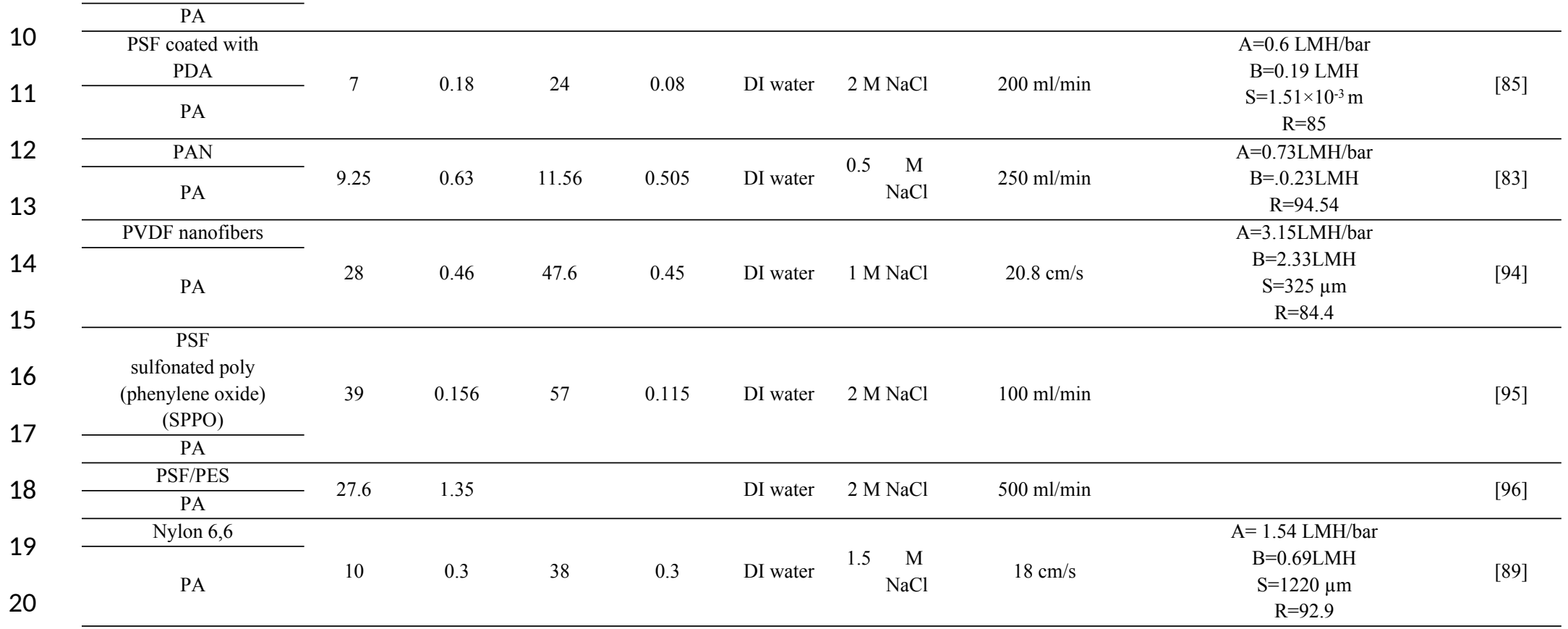

21 
Table1:(continued)

\begin{tabular}{|c|c|c|c|c|c|c|c|c|c|}
\hline \multirow{3}{*}{$\begin{array}{c}\text { support } \\
\text { Active layer }\end{array}$} & \multicolumn{4}{|c|}{ Performance } & \multicolumn{3}{|c|}{ Experimental condition } & \multirow[b]{3}{*}{$\begin{array}{c}\text { Intrinsic } \\
\text { properties }\end{array}$} & \multirow[b]{3}{*}{ ref } \\
\hline & \multicolumn{2}{|c|}{ AL-FS(FO) } & \multicolumn{2}{|c|}{ AL-DS(PRO) } & \multirow[b]{2}{*}{$\begin{array}{c}\text { Feed } \\
\text { solution }\end{array}$} & \multirow[b]{2}{*}{$\begin{array}{r}\text { Draw } \\
\text { solution }\end{array}$} & \multirow[b]{2}{*}{ cross-flow velocity } & & \\
\hline & $\begin{array}{r}\mathrm{J}_{\mathrm{v}} \\
(\mathrm{LMH}) \\
\end{array}$ & $\begin{array}{l}\mathrm{J}_{\mathrm{s}} / \mathrm{J}_{\mathrm{V}} \\
(\mathrm{g} / \mathrm{L})\end{array}$ & $\begin{array}{r}\mathrm{J}_{\mathrm{V}} \\
(\mathrm{LMH})\end{array}$ & $\begin{array}{l}\mathrm{J}_{\mathrm{s}} / \mathrm{J}_{\mathrm{v}} \\
(\mathrm{g} / \mathrm{L})\end{array}$ & & & & & \\
\hline PAN & & & & & & & & $\mathrm{A}=1.13 \mathrm{LMH} / \mathrm{bar}$ & \multirow[b]{2}{*}{ [97] } \\
\hline $\mathrm{PA}$ & 31.3 & 0.163 & & & DI water & $1 \mathrm{M} \mathrm{NaCl}$ & $750 \mathrm{ml} / \mathrm{min}$ & $\begin{array}{c}\mathrm{B}=0.335 \mathrm{LMH} \\
\mathrm{S}=112 \mu \mathrm{m} \\
\mathrm{R}=91.4\end{array}$ & \\
\hline PVDF nanofibers/ nylon 6,6 & \multirow[b]{2}{*}{30} & \multirow[b]{2}{*}{0.3} & \multirow[b]{2}{*}{41} & \multirow[b]{2}{*}{0.47} & \multirow[b]{2}{*}{ DI water } & \multirow{2}{*}{$\begin{array}{rr}1.5 & \mathrm{M} \\
\mathrm{NaCl}\end{array}$} & \multirow[b]{2}{*}{$18 \mathrm{~cm} / \mathrm{s}$} & $\mathrm{A}=1.28 \mathrm{LMH} / \mathrm{bar}$ & \multirow[b]{2}{*}{ [98] } \\
\hline (a) & & & & & & & & $\begin{array}{c}\mathrm{B}=0.25 \mathrm{LMH} \\
\mathrm{S}=193 \mu \mathrm{m}\end{array}$ & \\
\hline $\begin{array}{c}\begin{array}{c}\text { PSF/sulfonated } \\
\text { polysulfone (sPSF) }\end{array} \\
\text { PA }\end{array}$ & 29.02 & 0.18 & 49.92 & 0.18 & DI water & $1 \mathrm{M} \mathrm{NaCl}$ & $6.4 \mathrm{~cm} / \mathrm{s}$ & $\begin{array}{c}\mathrm{A}=1.93 \mathrm{LMH} / \mathrm{bar} \\
\mathrm{B}=0.31 \mathrm{LMH} \\
\mathrm{S}=220 \mu \mathrm{m}\end{array}$ & [99] \\
\hline $\begin{array}{c}\text { PES } \\
\text { SPES } \\
\text { PA }\end{array}$ & 35.1 & 0.28 & 42.1 & 0.26 & DI water & $2 \mathrm{M} \mathrm{NaCl}$ & & $\begin{array}{c}\mathrm{A}=2.9 \mathrm{LMH} / \mathrm{bar} \\
\mathrm{B}=5.1 \times 10^{-8} \mathrm{~m} / \mathrm{s} \\
\mathrm{S}=245 \mu \mathrm{m} \\
\mathrm{R}=91.1\end{array}$ & {$[100]$} \\
\hline $\begin{array}{l}\begin{array}{l}\text { PVDF nanofibers coated with } \\
\text { PVA (Polyvinyl alcohol) }\end{array} \\
\text { PA }\end{array}$ & 24.8 & 0.13 & 32.5 & 0.13 & DI water & $\begin{array}{r}0.5 \mathrm{M} \\
\mathrm{NaCl}\end{array}$ & $\begin{array}{l}13.88 \mathrm{~cm} / \mathrm{s} \\
500 \mathrm{ml} / \mathrm{min}\end{array}$ & $\begin{array}{c}\mathrm{A}=0.62 \mathrm{LMH} / \mathrm{bar} \\
\mathrm{B}=0.31 \mathrm{LMH} \\
\mathrm{S}=154 \mu \mathrm{m}\end{array}$ & {$[101]$} \\
\hline $\begin{array}{c}\text { PAN/PSF nanofibers } \\
\text { PA }\end{array}$ & & & 38.3 & 0.27 & DI water & $1 \mathrm{M} \mathrm{NaCl}$ & & $\begin{array}{c}\mathrm{A}=3.68 \mathrm{LMH} / \mathrm{bar} \\
\mathrm{B}=0.32 . \mathrm{LMH} \\
\mathrm{S}=34 \mu \mathrm{m}\end{array}$ & {$[102]$} \\
\hline CTA & & & & & & & & $\mathrm{A}=1.52 \mathrm{LMH} / \mathrm{bar}$ & \\
\hline PA & 11.79 & 0.58 & 14.58 & 0.69 & DI water & $\begin{array}{r}0.5 \mathrm{M} \\
\mathrm{NaCl}\end{array}$ & $333.3 \mathrm{ml} / \mathrm{min}$ & $\begin{array}{c}\mathrm{B}=0.27 \mathrm{LMH} \\
\mathrm{S}=516 \mu \mathrm{m} \\
\mathrm{R}=96\end{array}$ & {$[103$} \\
\hline $\begin{array}{c}\text { PES } \\
\text { PA } \\
\text { 2,5-disulfoaniline disodium } \\
\text { salt (DSA-2Na) }\end{array}$ & 12.6 & 0.126 & 16 & 0.11 & DI water & $\begin{array}{rr}0.5 & \mathrm{M} \\
\mathrm{NaCl}\end{array}$ & $1.3 \mathrm{~cm} / \mathrm{s}$ & $\begin{array}{c}\mathrm{A}=0.71 \mathrm{LMH} / \mathrm{bar} \\
\mathrm{B}=0.094 \mathrm{LMH} \\
\mathrm{R}=93.79\end{array}$ & {$[104]$} \\
\hline
\end{tabular}


Table1:(continued)

\begin{tabular}{|c|c|c|c|c|c|c|c|c|c|}
\hline support & \multicolumn{4}{|c|}{ Performance } & \multicolumn{3}{|c|}{ Experimental condition } & \multirow[b]{3}{*}{$\begin{array}{c}\text { Intrinsic } \\
\text { properties }\end{array}$} & \multirow{3}{*}{ ref } \\
\hline & \multicolumn{2}{|c|}{ AL-FS(FO) } & \multicolumn{2}{|c|}{ AL-DS(PRO) } & \multirow[b]{2}{*}{$\begin{array}{c}\text { Feed } \\
\text { solution }\end{array}$} & \multirow[b]{2}{*}{$\begin{array}{r}\text { Draw } \\
\text { solution }\end{array}$} & \multirow{2}{*}{ cross-flow velocity } & & \\
\hline Active layer & $\begin{array}{r}\mathrm{J}_{\mathrm{v}} \\
(\mathrm{LMH})\end{array}$ & $\begin{array}{l}\mathrm{J}_{\mathrm{s}} / \mathrm{J}_{\mathrm{v}} \\
(\mathrm{g} / \mathrm{L})\end{array}$ & $\begin{array}{r}\mathrm{J}_{\mathrm{v}} \\
(\mathrm{LMH})\end{array}$ & $\begin{array}{l}\mathrm{J}_{\mathrm{S}} / \mathrm{J}_{\mathrm{v}} \\
(\mathrm{g} / \mathrm{L})\end{array}$ & & & & & \\
\hline CA/PVDF nanofibers & \multirow[b]{2}{*}{31.3} & \multirow[b]{2}{*}{0.03} & & & \multirow[b]{2}{*}{ DI water } & \multirow{2}{*}{$\begin{array}{cc}0.5 & \mathrm{M} \\
& \mathrm{NaCl}\end{array}$} & \multirow{2}{*}{$\begin{array}{l}400 \mathrm{ml} / \mathrm{min} \\
13.88 \mathrm{~cm} / \mathrm{s}\end{array}$} & $\mathrm{A}=2.79 \mathrm{LMH} / \mathrm{bar}$ & \multirow[b]{2}{*}[105]{} \\
\hline PA & & & & & & & & $\begin{array}{c}\mathrm{B}=0.07 \mathrm{LMH} \\
\mathrm{S}=190 \mu \mathrm{m}\end{array}$ & \\
\hline $\begin{array}{l}\text { Poly (vinyl butyral) } \\
\text { (PVB) / poly (vinyl } \\
\text { chloride) (PVC) } \\
\text { PA }\end{array}$ & 29.37 & 0.11 & 49.85 & 0.1 & DI water & $1 \mathrm{M} \mathrm{NaCl}$ & $13.33 \mathrm{~cm} / \mathrm{s}$ & $\begin{array}{c}\mathrm{A}=1.75 \mathrm{LMH} / \mathrm{bar} \\
\mathrm{B}=8.99 \times 10^{-8} \mathrm{~m} / \mathrm{s} \\
\mathrm{S}=186 \mu \mathrm{m} \\
\mathrm{R}=94.98\end{array}$ & {$[106]$} \\
\hline PSF & \multirow[b]{2}{*}{25} & \multirow[b]{2}{*}{0.18} & \multirow[b]{2}{*}{54.5} & \multirow[b]{2}{*}{0.22} & \multirow[b]{2}{*}{ DI water } & \multirow[b]{2}{*}{$2 \mathrm{M} \mathrm{NaCl}$} & \multirow[b]{2}{*}{$1000 \mathrm{ml} / \mathrm{min}$} & $\mathrm{A}=1.51 \mathrm{LMH} / \mathrm{bar}$ & \multirow[b]{2}{*}{ [107] } \\
\hline $\begin{array}{c}\text { PA } \\
\text { polyethylenimine } \\
(\text { PEI })\end{array}$ & & & & & & & & $\begin{array}{c}\mathrm{B}=0.31 \mathrm{LMH} \\
\mathrm{S}=334.1 \mu \mathrm{m} \\
\mathrm{R}=85.1\end{array}$ & \\
\hline PAN & \multirow[b]{2}{*}{34.2} & \multirow[b]{2}{*}{0.17} & \multirow[b]{2}{*}{44.5} & \multirow[b]{2}{*}{0.19} & \multirow[b]{2}{*}{ DI water } & \multirow[b]{2}{*}{$1 \mathrm{M} \mathrm{NaCl}$} & \multirow[b]{2}{*}{$600 \mathrm{ml} / \mathrm{min}$} & $\mathrm{A}=3.26 \mathrm{LMH} / \mathrm{bar}$ & \multirow[b]{2}{*}[108]{} \\
\hline $\begin{array}{c}\text { PA } \\
\mathrm{PA}^{*} \text { (toluene as an } \\
\text { organic solvent) } \\
\end{array}$ & & & & & & & & $\begin{array}{c}\mathrm{B}=0.25 \mathrm{LMH} \\
\mathrm{S}=378 \mu \mathrm{m} \\
\mathrm{R}=98.1\end{array}$ & \\
\hline $\begin{array}{c}- \\
\text { PA }\end{array}$ & 80.54 & 0.44 & & & DI water & $1 \mathrm{M} \mathrm{NaCl}$ & & & [109] \\
\hline $\begin{array}{c}\text { Cyanoethyl Cellulose } \\
\text { (CEC) }\end{array}$ & 9.10 & 0.15 & 20.67 & 0.11 & DI water & $1 \mathrm{M} \mathrm{NaCl}$ & $480 \mathrm{~mL} / \mathrm{min}$ & $\begin{array}{c}\mathrm{A}=1.17 \mathrm{LMH} / \mathrm{bar} \\
\mathrm{B}=0.2 \mathrm{LMH} \\
\mathrm{S}=922 \mu \mathrm{m} \\
\end{array}$ & [110] \\
\hline $\begin{array}{c}\text { PES/ } \\
\text { sulfonated } \\
\text { polyetheretherketone } \\
\text { (sPEEK) }\end{array}$ & 11 & 1.06 & 23 & 1.5 & DI water & $\begin{array}{r}0.5 \mathrm{M} \\
\mathrm{NaCl}\end{array}$ & $250 \mathrm{ml} / \mathrm{min}$ & $\begin{array}{c}\mathrm{A}=1.9 \mathrm{LMH} / \mathrm{bar} \\
\mathrm{S}=383 \mu \mathrm{m} \\
\mathrm{R}=75\end{array}$ & [111] \\
\hline PES & & & & & & & & $\mathrm{A}=2.64 \mathrm{LMH} / \mathrm{bar}$ & \\
\hline PA & 21.6 & 0.77 & 29.3 & 0.66 & DI water & $1 \mathrm{M} \mathrm{NaCl}$ & $8 \mathrm{~cm} / \mathrm{s}$ & $\begin{array}{c}\mathrm{B}=2.35 \mathrm{LMH} \\
\mathrm{R}=82.53\end{array}$ & [112] \\
\hline DPE & & & & & & & & $\mathrm{A}=6.7 \mathrm{LMH} / \mathrm{bar}$ & \\
\hline $\begin{array}{l}\mathrm{PA}^{*} \text { (toluene as an } \\
\text { organic solvent) }\end{array}$ & 53 & 0.28 & 64.8 & 0.28 & DI water & $1 \mathrm{M} \mathrm{NaCl}$ & $600 \mathrm{ml} / \mathrm{min}$ & $\begin{array}{c}\mathrm{B}=0.68 \mathrm{LMH} \\
\mathrm{S}=168 \mu \mathrm{m} \\
\mathrm{R}=98.1\end{array}$ & [92] \\
\hline
\end{tabular}


Table1:(continued)

\begin{tabular}{|c|c|c|c|c|c|c|c|c|c|}
\hline \multirow{3}{*}{$\begin{array}{c}\text { support } \\
\text { Active layer }\end{array}$} & \multicolumn{4}{|c|}{ Performance } & \multicolumn{3}{|c|}{ Experimental condition } & \multirow[b]{3}{*}{$\begin{array}{c}\text { Intrinsic } \\
\text { properties }\end{array}$} & \multirow[b]{3}{*}{ ref } \\
\hline & \multicolumn{2}{|c|}{ AL-FS(FO) } & \multicolumn{2}{|c|}{ AL-DS(PRO) } & \multirow[b]{2}{*}{$\begin{array}{r}\text { Feed } \\
\text { solution }\end{array}$} & \multirow[b]{2}{*}{$\begin{array}{r}\text { Draw } \\
\text { solution }\end{array}$} & \multirow[b]{2}{*}{ cross-flow velocity } & & \\
\hline & $\begin{array}{r}\mathrm{J}_{\mathrm{v}} \\
(\mathrm{LMH})\end{array}$ & $\begin{array}{l}\mathrm{J}_{\mathrm{s}} / \mathrm{J}_{\mathrm{v}} \\
(\mathrm{g} / \mathrm{L})\end{array}$ & $\begin{array}{r}\mathrm{J}_{\mathrm{v}} \\
(\mathrm{LMH})\end{array}$ & $\begin{array}{l}\mathrm{J}_{\mathrm{s}} / \mathrm{J}_{\mathrm{v}} \\
(\mathrm{g} / \mathrm{L})\end{array}$ & & & & & \\
\hline PES & & & \multirow{3}{*}{15.79} & \multirow{3}{*}{0.26} & \multirow{3}{*}{$\begin{array}{c}1,000 \\
\text { ppm of } \\
\text { oil } \\
\text { emulsion }\end{array}$} & \multirow{3}{*}{$2 \mathrm{M} \mathrm{NaCl}$} & \multirow{3}{*}{$32.72 \mathrm{~cm} / \mathrm{s}$} & & \multirow{3}{*}[113]{} \\
\hline zwitterion & & & & & & & & $\begin{array}{c}\mathrm{A}=0.5 / \mathrm{LMH} / \mathrm{bar} \\
\mathrm{B}=0.45 \mathrm{LMH}\end{array}$ & \\
\hline PA & & & & & & & & $\mathrm{R}=95.8$ & \\
\hline PSF & \multirow{3}{*}{25.5} & \multirow{3}{*}{0.19} & & & \multirow{3}{*}{ DI water } & \multirow{3}{*}{$2 \mathrm{M} \mathrm{NaCl}$} & \multirow{3}{*}{$8.5 \mathrm{~cm} / \mathrm{s}$} & $\mathrm{A}=2.44 \mathrm{LMH} / \mathrm{bar}$ & \multirow{3}{*}{114} \\
\hline PA & & & & & & & & $\mathrm{B}=0.42 \mathrm{LMH}$ & \\
\hline $\mathrm{CaCl}_{2}$ & & & & & & & & $\mathrm{R}=97.9$ & \\
\hline cellulose acetate & \multirow{4}{*}{16.8} & \multirow{4}{*}{0.35} & \multirow{4}{*}{27.5} & \multirow{4}{*}{0.38} & \multirow{4}{*}{ DI water } & \multirow{4}{*}{$1 \mathrm{M} \mathrm{NaCl}$} & \multirow{4}{*}{$1666 \mathrm{ml} / \mathrm{min}$} & $\mathrm{A}=1.08 \mathrm{LMH} / \mathrm{bar}$ & \multirow{4}{*}{ [115] } \\
\hline butyrate $(\mathrm{CAB})$ and & & & & & & & & $\mathrm{B}=0.529 \mathrm{LMH}$ & \\
\hline PVB & & & & & & & & $\mathrm{S}=363.5 \mu \mathrm{m}$ & \\
\hline PA & & & & & & & & $\mathrm{R}=86.6$ & \\
\hline PSF & \multirow{2}{*}{73.87} & \multirow[b]{2}{*}{0.11} & \multirow[b]{2}{*}{120.1} & \multirow[b]{2}{*}{0.1} & \multirow[b]{2}{*}{ DI water } & \multirow[b]{2}{*}{$2 \mathrm{M} \mathrm{NaCl}$} & \multirow[b]{2}{*}{$300 \mathrm{ml} / \mathrm{min}$} & $\mathrm{A}=3.62 \mathrm{LMH} / \mathrm{bar}$ & \\
\hline $\begin{array}{c}\text { PA* (under }^{*} \\
\text { ultrasonication) }\end{array}$ & & & & & & & & $\begin{array}{c}\mathrm{B}=0.16 \mathrm{LMH} \\
\mathrm{R}=97.1\end{array}$ & [116] \\
\hline
\end{tabular}

${ }^{*}$ The majority of PA active layers that presented in table 1 were fabricated with conventional interfacial polymerization that was explained in section 2.2 except the ones have been highlighted by asterisk. 


\section{4. $\quad$ Performance of TFC-A Hollow fiber membranes}

3 Hollow fiber (HF) membranes can be more suitable for FO process than thin flat sheet membranes 4 because of their self-supported mechanical properties, since it allows denser packing density and 5 higher effective membrane surface area for both feed and draw solutions [117]. Moreover, 6 fabricating the module of the hollow fibers is relatively easy and the module provides greater 7 surface area per volume ratio. It should also be noted that the structure of hollow fibers may offer 8 the flow pattern specifically required for FO processes [117-121]. Nonetheless, hollow fiber 9 membranes manufacturing techniques suffer from a few challenges, such as restriction of 10 appropriate materials, i.e. hydrophilic and tough materials, as well as necessity to optimize the 11 phase separation techniques for the fabrication of hollow fibers with enhanced mechanical quality 12 and separation properties, while maintaining enough low structural parameter [122].

13 Fig. 8 shows schematically a dry-jet wet spinning of hollow fiber membranes. Readers can refer to the literature for the more details.

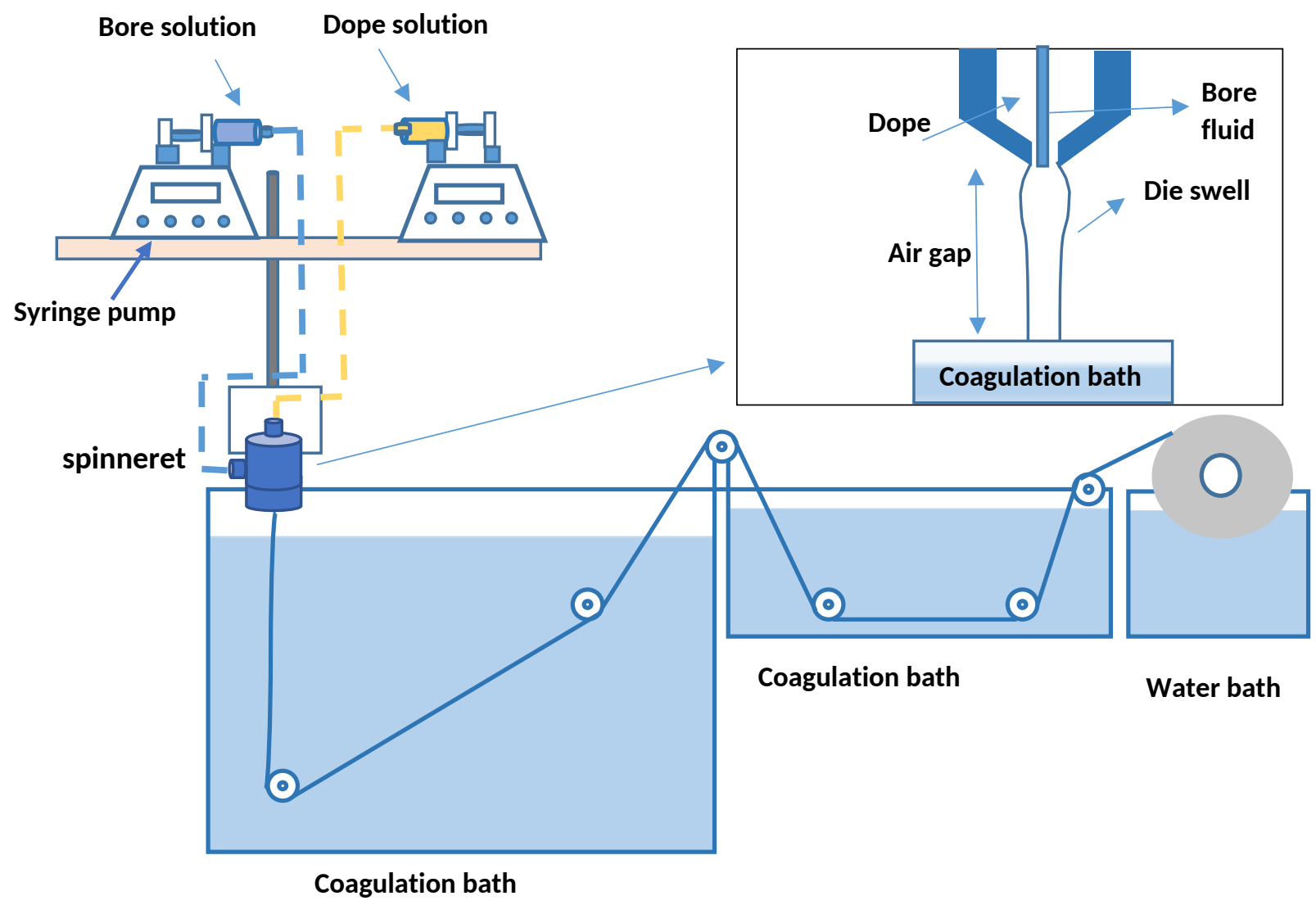

Fig 8: Schematic of dry-jet wet-spinning of hollow fiber membranes 


\subsubsection{PES as substrate}

Wang et al. [119] produced TFC-A hollow fiber membranes with PES substrates and compared them with commercial HTI flat sheet membranes and also commercial NF hollow fiber membranes when applied in an FO unit. In their experiments, two substrates, A and B, were spun. For A, a mixture of water and NMP was used as the bore fluid and tap water was used as the external coagulant, while for B only water was used for both internal and external coagulant. The active layer was synthesized onto the outer surface of the substrate A while the active layer was synthesized in the inner surface of substrate B. Both substrates had a sponge-like and a porous structure. However, the contribution of the sponge-like structure was lower in substrate B, and hence B exhibited a higher water flux. They have considered that the structural parameter, $S$, of the membrane is a series combination of the sponge-like and porous structure, as shown in Eq. (1), with the sponge-like structure of greater contribution to $S$. Since the substrate B has smaller sponge-like fraction, smaller $S$ and less internal concentration polarization, the substrate B is preferable. However, they did not explain, it can be found out from the SEM results, that the "pore" here refers to the finger-like structure.

$$
S=\frac{\tau_{\text {sponge }} l_{\text {sponge }}}{\varepsilon_{\text {sponge }}}+\frac{\tau_{\text {pore }} l_{\text {pore }}}{\varepsilon_{\text {pore }}}
$$

Furthermore, they have discussed on the bore shrinkage of the substrate membrane B during the hollow fiber drying, which increases the fluid flow resistance in the bore. It could be prevented by replacing the water in the membrane pore with water/glycerol mixture before drying. The optimal membrane made in this study (membrane B without bore shrinkage) showed a better performance compared to the HTI commercial flat sheet membrane.

Shi et al. [123] also studied the effect of the pore size of the hollow fiber substrate on the active layer properties of TFC membranes and their FO performance. After PES hollow fiber substrates were spun by the dry-jet wet method, polyamide active layer was formed onto the lumen side of substrates. They found that defective active layer is formed more readily when the substrate pore size is large. This result coincides with the earlier study of Singh et al. [91] on the mechanism of the active layer formation using two substrates with different pore sizes. Fig. 9 shows schematically the skin layer formation mechanism proposed by Singh et al. [91] For type1 substrate with smaller pore sizes, monomer does not penetrate into the pore easily and thus the polyamide active layer is formed only onto the substrate surface. However, in the case of the substrate type 2 with larger pore sizes, monomer penetrates into the substrate pore and thus a part of the active layer is formed inside the pore, reducing the active layer thickness above the substrate surface and thus increasing the chance of defect formation. 


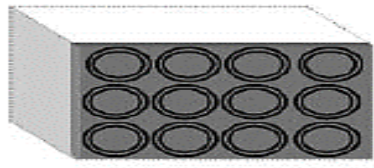

Front view

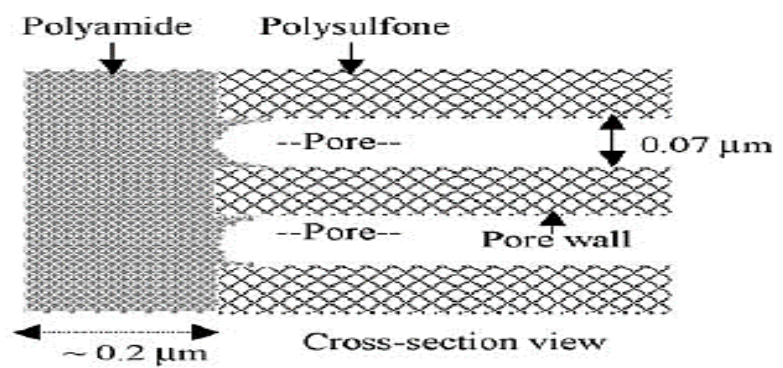

Type 1

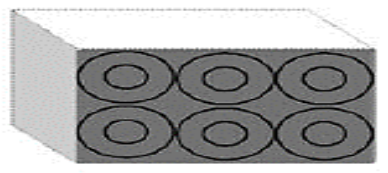

Front view

Fig 9: Formation of polyamide layer on the poly sulfone layer for two substrates with different pore sizes [91]

Sukitpaneenit and Chung [124] produced a polyamide TFC hollow fiber membrane with PES substrate using a two-layer coextrusion, as shown in Fig. 10. From the figure, the nonsolvent bore fluid (either (a) water, (b) water/NMP, or (c) water/NMP/PEG), polymer dope and NMP are extruded from the central tube, the inner channel and the external channel, respectively. The water containing bore fluid facilitates the phase inversion process, thus forming a dense skin layer at the inner surface, while delayed phase inversion caused by NMP makes the outer surface more porous. Hence the hollow fiber membrane shows an asymmetric structure as shown in Fig. 11. The polymer dope consisted of PES, NMP, water, and PEG. Addition of water increases the dope viscosity and also brings the dope composition near the binodal line, both contributing to the prevention of macro voids formation. However, PEG acts as a pore former, it also prevents the formation of macro voids. The internal surface roughness is controlled by the property of the internal coagulant, e.g. instantaneous demixing caused by water makes the internal surface the roughest among the three different internal coagulants. In addition, the membrane contained a mixture of finger-like structure, sponge-like structure and macro voids (not shown in Fig. 11).

In the earlier works $[119,125]$, it has been stated that an ideal substrate for TFC hollow fiber membranes should include more finger-like structure and large pores even though some small parts of the cross-section are allowed to be occupied by sponge-like structure. However in this work [124], even though a large part of the substrate is occupied by sponge-like structure instead of finger-like pores (see Fig. 11), this substrate was found equally suitable to reduce ICP and to achieve a high water flux. 


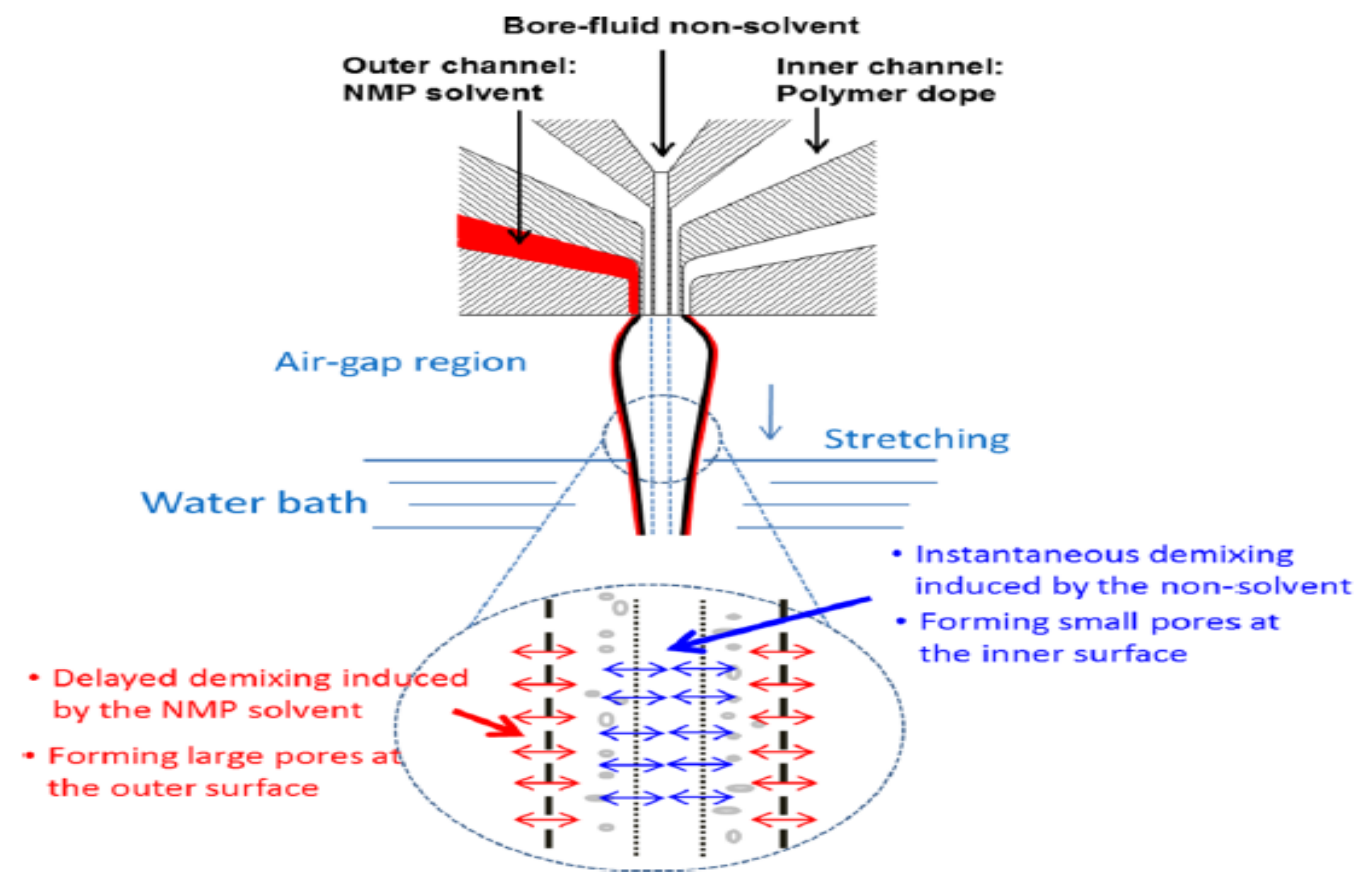

Fig 10: Phase inversion process with the aid of coextrusion technique employing a dual-layer spinneret [124] 


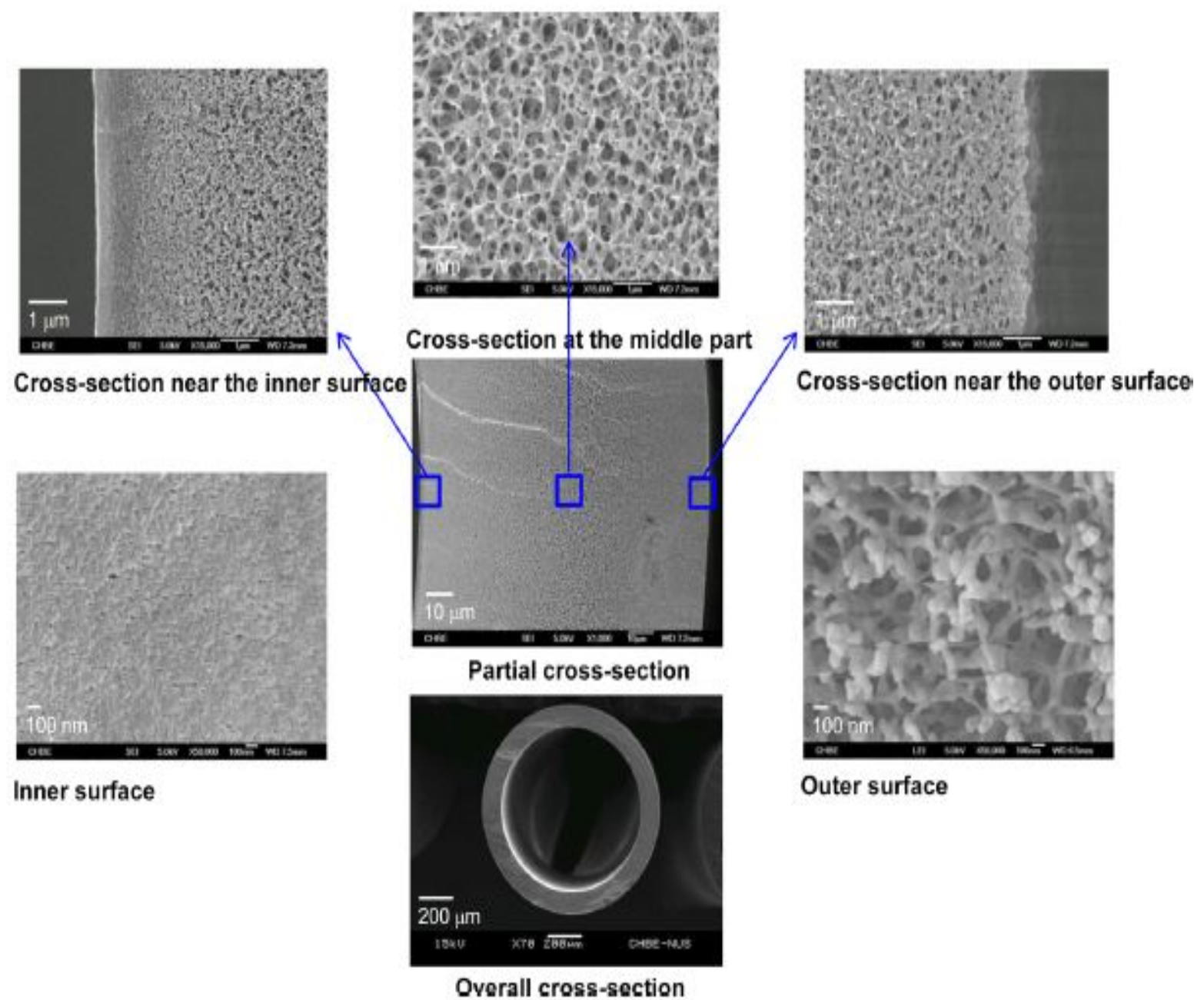

Fig 11: SEM micrographs of different bulk and surface morphologies of PES hollow fiber membrane supports [124]

Lim et al. [126] fabricated a defect free outer selective layer hollow fiber membrane with PES substrate. The advantages of making the active layer on the outer surface of the substrate are i) it provides a larger surface area per fiber and ii) it offers an easier fouling control under FO mode and lower fouling propensity. Also, they studied the effect of air gap on the performance of the FO membranes. The air-gap distance was in the range of $2-8 \mathrm{~cm}$. At the lowest air-gap the membrane substrate showed the highest porosity, the largest fiber diameter and the largest mean pore size compared to the other samples. These properties (with the lowest air gap) related to low elongation stress on the fibers in the air-gap region. Thus, a thicker and rougher active layer formed on the substrate with lowest air gap. Eventually, a defective active layer was formed on this substrate. When the surface pore size is large, the MPD solution cannot fill the pores uniformly and can be diffuse inside the pores or may be removed by air-blowing because of weak surface tension. These phenomena have also been reported by the other researchers [91, 123]. 


\subsubsection{Polyphenylene sulfone as substrate}

Zhong et al. [120] studied the effects of degree of sulfonation of a polyphenylene sulfone substrate on the performance of hollow fiber polyamide TFC-A membrane. In this study, the active layer was synthesized onto the membrane lumen surface. The advantages of making the active layer onto the inner surface of substrate are i) a better distribution of feed when feed flows through the lumen side, ii) easy protection of the defect-free active layer and, iii) low resistance against water permeation. Also, when feed flows in the lumen side, no dead space is created. On the other hand, when the feed flows in the shell side, the non-ideal fluid passage leads to creation of dead spaces, hence severe concentration polarization is experienced [127]. Eventually, Zhong et al. [120] obtained the following results:

1) By increasing the degree of sulfonation, i.e. the higher the sulfonate concentration in the substrate, the substrate tended toward sponge-like structure formation and the thickness of the hollow fiber increased due to the increase in viscosity i.e. delayed demixing. Conversely, the lower the sulfonate concentration in the substrate, the less viscosity of the dope solution. As a result, faster precipitation was experienced and larger pores were formed.

2) Degree of sulfonation showed a direct correlation to the membrane hydrophilicity.

3) As the degree of sulfonation increased, the water flux increased as well. Because the sulfonation tended to encourage the formation of sponge-like structure, it was concluded that the effect of hydrophilicity on flux was more pronounced than the effect of structure as mentioned earlier by other researchers [81]. $3.5 \% \mathrm{NaCl}$ solution as feed and 2 molar $\mathrm{NaCl}$ solution as draw solution in AL-DS (PRO) orientation using a membrane with a sulfonate content. By reducing the fibers lengths, the effect of internal and external concentration polarization decreased and therefore the water flux was increased. The effect of the fiber length on reverse salt flux was not reported in this study; however, the effect of the fiber length on the membrane performance is a controversial issue that needs further investigation.

\subsubsection{Matrimid $^{\circledR}$ as substrate}

Luo et al. [121] designed a new TFC-A hollow fiber membrane consisting of Matrimid ${ }^{\circledR}$ substrate and active polyamide layer for FO. Matrimid ${ }^{\circledR}$ is a commercial aromatic polyimide that is soluble in common organic solvents [129]. The active layer was made, again, onto the inner surface of the membrane. The spinneret geometry had a blossom geometry with three separate bore flows (Fig. 12). The tri-needle spinneret had three separate needles placed inside it uniformly. This special spinneret design was made to address the concern over the long-term stability and durability of the potting (seal) of hollow fibers spun from the single-needle spinneret. Indeed, the hollow fibers spun by a multi bore spinneret showed better durability during the long term operations and mechanical washing [130]. Fig. 13 schematically shows the two different hollow fiber cross sections. Between those, triangular geometry (Fig. 13 left) was suggested for balancing the mechanical resistance and water flux. The triangular geometry also showed better module 
performance because of the higher packing density, effective surface area of membrane and water flux. In contrast to triangular geometry, circular geometry shows disadvantages such as the uneven thickness of the walls, low mechanical resistance of the thin walls, and high-water permeation resistance of the thick walls. The key parameters in creating the triangular geometry were closeto-gelation dope formation, the flow rate of the dope solution, the air gap, and the characteristics of the bore fluid and the coagulant [121]. Despite the advantages they mentioned, when feed flows in the shell side, triangular shape may result in severe fouling between the fibers.
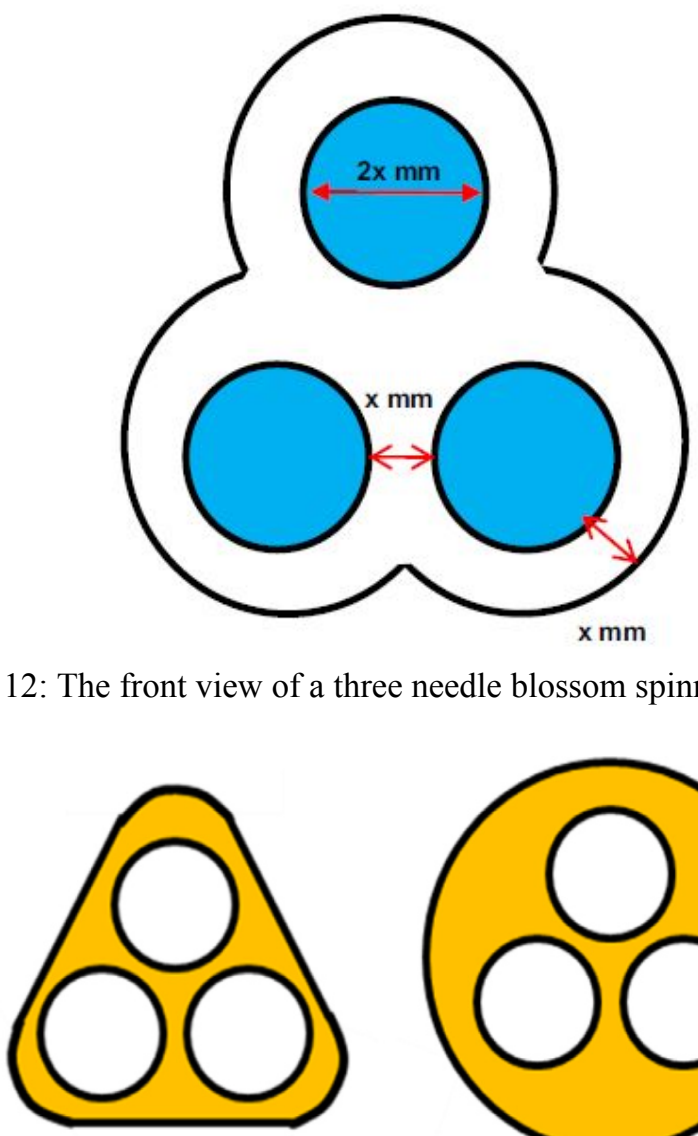

Fig 12: The front view of a three needle blossom spinneret [130]

Ren et al. [131] produced a TFC hollow fiber membranes with PAN substrate and polyamide active layer. PAN spins easily and is inherently hydrophilic. In contrast to the other works, the polyamide active layer was at the outer surface, which showed less fouling tendency compared to the membranes in which the active layer was at the inner surface. Usually, it is more difficult to form

21 the inner surface of hollow fiber, especially for large-scale production. Conversely, Zhong [120] 
outer surface. The selection of inner or outer selective layer can be related to the operation condition, i.e. the type of feed and/or draw solutions. It is well known that the formation of defect free active layer depends on how well the aqueous MPD solution is removed before the substrate is immersed in the organic phase. In the case of the flat sheet membrane, aqueous solution is removed by a roller. The aqueous solution in the lumen of hollow fiber can be blown by air. To form the active layer onto the outer surface of hollow fiber, the interfacial polymerization should be carried out continuously, i.e. the hollow fiber should pass through aqueous solution, air, and organic solution chamber progressively. The method has some problems, namely (i) the membrane outer surface contacts with the moving surface of the pulley during the interfacial polymerization creating an imperfect layer. (ii) the continuous flow of thousands of meters of fibers in large-scale production is time-consuming. (iii) because of many controlling parameters the process is complex. Therefore, the active layer formation onto the outer surface was proposed to be done not for a single fiber but for multiple of hollow fibers all at once [118]. But even in this case, the interfacial polymerization may take place between two fibers, if the aqueous solution is not well removed.

In Ren et al. [131] work, a batch method was used. Three substrate hollow fibers were spun with the bore fluids of three different DMF contents, ranging from 0 to $60 \%$, in the DMF/water mixture. In Fig. 14, the cross-sectional FESEM images of the substrates are shown with two different magnifications. All the three substrates had a flat and smooth surface that was necessary for forming strong and defect free active layer. As DMF concentration in bore fluid increases, the rate of polymer solidification during the phase inversion is delayed, resulting in thinner hollow fiber wall with more finger-like pores.

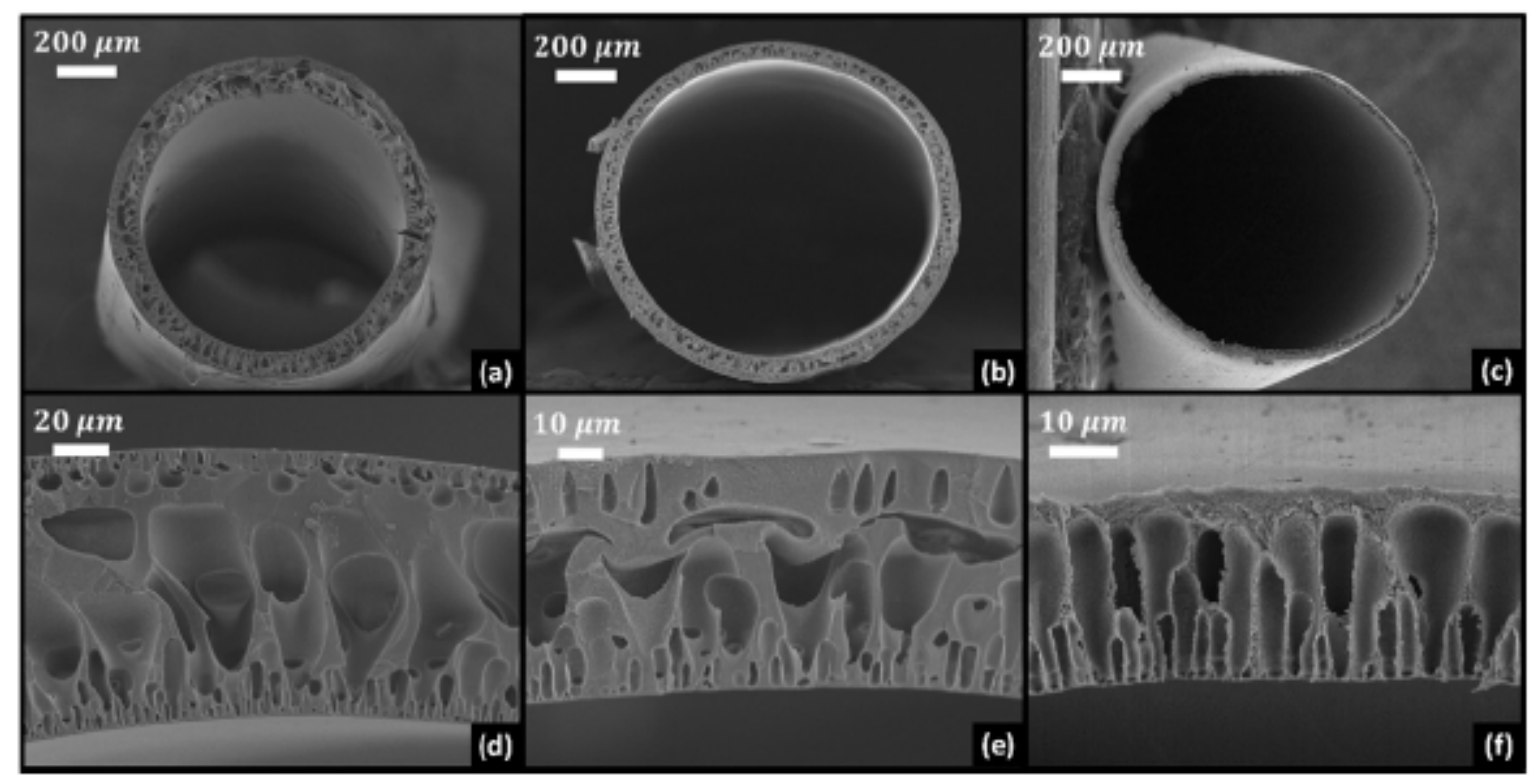

Fig 14: Cross-sectional FESEM images of hollow fiber substrates. (a), (b) and (c) PAN-0, PAN-30 and PAN-60 at $65 \times$, respectively. (d) PAN-0 at 600×, (e) PAN-30 at 950×, (f) PAN-60 at 1000× [131]

\subsubsection{Polyketone as substrate}


Shibuya et al. [132] prepared TFC-A hollow fiber membranes on the membrane performance with two different substrates, both made of Polyketone. Polyketone possesses good mechanical and chemical properties and shows good behavior over a wide range of temperature. As a drawback, polyketone is not dissolved in organic solvents [133]. They formed the active layer on the outer surface by successive immersion of hollow fibers in the aqueous and organic solutions (see Fig. 15). Both ends of the substrates were sealed by epoxy resin to prevent the solution from entering 7 into the lumen side of the membranes [132].

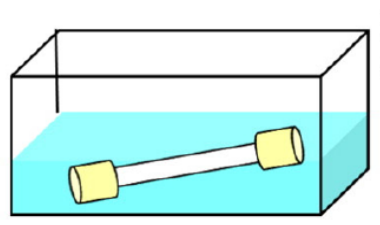

Immersion in Aqueous MPD solution

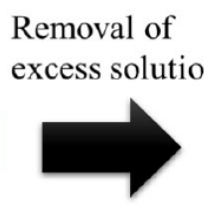

(1)

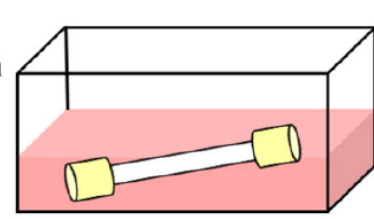

Immersion in Hexane TMC solution

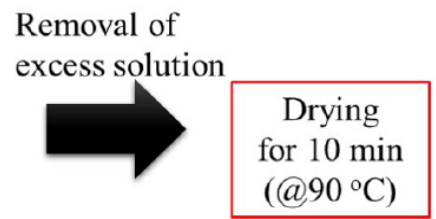

(@, $\left.90^{\circ} \mathrm{C}\right)$

Fig 15: Steps of the interfacial polymerization at the shell side of the hollow fiber membranes [132]

10

11

12

13

14

15

As shown in Table 2, the two substrates, HF-A and HF-B, were very different in diameters and thicknesses but similar in mean pore size and porosity and both possessed microfiltration structure [132].

Table 2: HF support membrane characteristics [132]

\begin{tabular}{|c|c|c|c|c|c|}
\hline sample & $\begin{array}{l}\text { Inner diameter } \\
(\mu \mathrm{m})\end{array}$ & $\begin{array}{l}\text { Outer diameter } \\
\qquad(\mu \mathrm{m})\end{array}$ & $\begin{array}{c}\text { Thickness } \\
(\mu \mathrm{m})\end{array}$ & $\begin{array}{l}\text { Mean pore size } \\
(\mathrm{nm})\end{array}$ & $\begin{array}{l}\text { Porosity } \\
(\%)\end{array}$ \\
\hline HF-A & 347 & 480 & 66.5 & 41.4 & 73.6 \\
\hline HF-B & 609 & 893 & 142 & 47.8 & 78.0 \\
\hline
\end{tabular}

Water flux and reverse salt flux of HF-A membrane were higher than those of HF-B membrane despite the smaller porosity of HF-A. This is because of the thinner wall of HF-A. As the draw solution concentration increased both water flux and reverse salt flux increased but specific reverse salt flux remained unchanged. They stated that, this refers to the fact that, specific reverse salt flux is an intrinsic property of the active layer [132].

The works published in the literature on hollow fiber TFC-A membranes are summarized in Table 3. The table includes water flux and specific reverse salt flux with a polyamide active layer in the two different modes of AL-FS(FO) and AL-DS(PRO). 
Table 3: Summary of the studies on TFC-A hollow fiber FO membranes

\begin{tabular}{|c|c|c|c|c|c|c|c|c|c|c|}
\hline \multirow{3}{*}{$\begin{array}{c}\text { support } \\
\text { Active layer } \\
\text { Active layer place }\end{array}$} & \multicolumn{4}{|c|}{ Performance } & \multicolumn{4}{|c|}{ Experimental condition } & \multirow{3}{*}{$\begin{array}{c}\text { Intrinsic } \\
\text { properties }\end{array}$} & \multirow{3}{*}{ ref } \\
\hline & \multicolumn{2}{|c|}{ AL-FS(FO) } & \multicolumn{2}{|c|}{ AL-DS(PRO) } & \multirow{2}{*}{$\begin{array}{c}\text { Feed } \\
\text { solution }\end{array}$} & \multirow{2}{*}{$\begin{array}{r}\text { Draw } \\
\text { solution }\end{array}$} & \multirow{2}{*}{$\begin{array}{c}\text { cross-flow velocity } \\
\text { or } \\
\text { Reynolds number } \\
\text { Lumen side }\end{array}$} & \multirow{2}{*}{$\begin{array}{c}\text { cross-flow velocity } \\
\text { or } \\
\text { Reynolds number } \\
\text { Shell side }\end{array}$} & & \\
\hline & $\begin{array}{r}\mathrm{J}_{\mathrm{v}} \\
(\mathrm{LMH})\end{array}$ & $\begin{array}{l}\mathrm{J}_{\mathrm{s}} / \mathrm{J}_{\mathrm{v}} \\
(\mathrm{g} / \mathrm{L})\end{array}$ & $\begin{array}{r}\mathrm{J}_{\mathrm{v}} \\
(\mathrm{LMH})\end{array}$ & $\begin{array}{l}\mathrm{J}_{\mathrm{s}} / \mathrm{J}_{\mathrm{v}} \\
(\mathrm{g} / \mathrm{L})\end{array}$ & & & & & & \\
\hline $\begin{array}{c}\text { PES } \\
\text { PA } \\
\text { inner surface }\end{array}$ & 14 & 0.13 & 32.2 & 0.11 & DI water & $\begin{array}{r}0.5 \mathrm{M} \\
\mathrm{NaCl}\end{array}$ & 1500 & 450 & $\begin{array}{c}\mathrm{A}=2.22 \mathrm{LMH} / \mathrm{bar} \\
\mathrm{B}=0.2 \mathrm{LMH} \\
\mathrm{S}=5.95 \times 10^{-4} \mathrm{~m} \\
\mathrm{R}=91\end{array}$ & {$[119]$} \\
\hline $\begin{array}{c}\text { PES } \\
\text { PA } \\
\text { inner surface } \\
\end{array}$ & 18.3 & 0.086 & 42.6 & 0.094 & DI water & $\begin{array}{lc}0.5 & \mathrm{M} \\
& \mathrm{NaCl}\end{array}$ & 2500 & 2500 & $\begin{array}{c}\mathrm{A}=3.2 \mathrm{LMH} / \mathrm{bar} \\
\mathrm{R}=92\end{array}$ & {$[125$} \\
\hline $\begin{array}{c}\text { PES } \\
\text { PA } \\
\text { inner surface }\end{array}$ & 17.6 & 0.068 & 49 & 0.081 & DI water & $\begin{array}{cc}0.5 & \mathrm{M} \\
& \mathrm{NaCl}\end{array}$ & $450 \mathrm{~mL} / \mathrm{min}$ & $1500 \mathrm{~mL} / \mathrm{min}$ & $\begin{array}{c}\mathrm{A}=3.93 \mathrm{LMH} / \mathrm{bar} \\
\mathrm{B}=0.27 \mathrm{LMH} \\
\mathrm{S}=0.51 \mathrm{~mm} \\
\mathrm{R}=90.4\end{array}$ & {$[123$} \\
\hline $\begin{array}{c}\text { PES } \\
\text { PA } \\
\text { inner surface }\end{array}$ & 32.1 & 0.2 & 57.1 & 0.12 & DI water & $2 \mathrm{M} \mathrm{NaCl}$ & $100 \mathrm{ml} / \mathrm{min}$ & $200 \mathrm{ml} / \mathrm{min}$ & $\begin{array}{c}\mathrm{A}=1.18 \mathrm{LMH} / \mathrm{bar} \\
\mathrm{B}=0.135 \mathrm{LMH} \\
\mathrm{S}=2.19 \times 10^{-4} \mathrm{~m} \\
\mathrm{R}=87.95\end{array}$ & {$[124$} \\
\hline $\begin{array}{c}\begin{array}{c}\text { sulfonated poly } \\
\text { phenylene sulfone } \\
\text { (sPPSU) }\end{array} \\
\text { PA } \\
\text { inner surface } \\
\end{array}$ & 22.51 & 0.24 & 49.39 & 0.22 & DI water & $\begin{array}{cc}0.5 & \mathrm{M} \\
& \mathrm{NaCl}\end{array}$ & $100 \mathrm{ml} / \mathrm{min}$ & $200 \mathrm{ml} / \mathrm{min}$ & $\begin{array}{c}\mathrm{A}=1.99 \mathrm{LMH} / \mathrm{bar} \\
\mathrm{B}=0.0399 \mathrm{LMH} \\
\mathrm{S}=1.63 \times 10^{-4} \mathrm{~m} \\
\mathrm{R}=90.9\end{array}$ & {$[120$} \\
\hline $\begin{array}{c}\text { PES } \\
\text { PA } \\
\text { cetyltrimethylammonium } \\
\text { chloride } \\
\text { (CTAC) }\end{array}$ & 5.32 & & & & DI water & $2 \mathrm{M} \mathrm{NaCl}$ & & & $\mathrm{R}=98$ & {$[134$} \\
\hline Outer surface & & & & & & & & & & \\
\hline $\begin{array}{c}\text { Matrimid } \\
\text { PA } \\
\text { inner surface }\end{array}$ & 11.8 & 0.21 & 50.5 & 0.07 & DI water & $2 \mathrm{M} \mathrm{NaCl}$ & $200 \mathrm{ml} / \mathrm{min}$ & $200 \mathrm{ml} / \mathrm{min}$ & $\begin{array}{c}\mathrm{A}=1.51 \mathrm{LMH} / \mathrm{bar} \\
\mathrm{B}=0.44 \mathrm{LMH} \\
\mathrm{S}=1.1 \times 10^{-3} \mathrm{~m}\end{array}$ & {$[121$} \\
\hline
\end{tabular}


Table 3: (continued)

\begin{tabular}{|c|c|c|c|c|c|c|c|c|c|c|}
\hline \multirow{3}{*}{$\begin{array}{c}\text { support } \\
\text { Active layer } \\
\text { Active layer place }\end{array}$} & \multicolumn{4}{|c|}{ Performance } & \multicolumn{4}{|c|}{ Experimental condition } & \multirow{3}{*}{$\begin{array}{l}\text { Intrinsic } \\
\text { properties }\end{array}$} & \multirow{3}{*}{ ref } \\
\hline & \multicolumn{2}{|c|}{ AL-FS(FO) } & \multicolumn{2}{|c|}{ AL-DS(PRO) } & \multirow{2}{*}{$\begin{array}{c}\text { Feed } \\
\text { solution }\end{array}$} & \multirow{2}{*}{$\begin{array}{r}\text { Draw } \\
\text { solution }\end{array}$} & \multirow{2}{*}{$\begin{array}{l}\text { cross-flow velocity } \\
\text { or } \\
\text { Reynolds number } \\
\text { Lumen side }\end{array}$} & \multirow{2}{*}{$\begin{array}{c}\text { cross-flow velocity } \\
\text { or } \\
\text { Reynolds number } \\
\text { Shell side }\end{array}$} & & \\
\hline & $\begin{array}{r}\mathrm{J}_{\mathrm{v}} \\
(\mathrm{LMH})\end{array}$ & $\begin{array}{l}\mathrm{J}_{\mathrm{S}} / \mathrm{J}_{\mathrm{v}} \\
(\mathrm{g} / \mathrm{L})\end{array}$ & $\begin{array}{r}\mathrm{J}_{\mathrm{v}} \\
(\mathrm{LMH})\end{array}$ & $\begin{array}{l}\mathrm{J}_{\mathrm{s}} / \mathrm{J}_{\mathrm{v}} \\
(\mathrm{g} / \mathrm{L})\end{array}$ & & & & & & \\
\hline PAN & \multirow{3}{*}{24.71} & \multirow{3}{*}{0.77} & \multirow{3}{*}{36.57} & \multirow{3}{*}{0.512} & \multirow{3}{*}{ DI water } & \multirow{3}{*}{$1 \mathrm{M} \mathrm{NaCl}$} & \multirow{3}{*}{1100} & \multirow{3}{*}{\multicolumn{2}{|c|}{$\begin{array}{c}\mathrm{A}=1.5 \mathrm{LMH} / \mathrm{bar} \\
\mathrm{B}=2.1 \mathrm{LMH}\end{array}$}} & \multirow{3}{*}{ [131] } \\
\hline PA & & & & & & & & & & \\
\hline outer surface & & & & & & & & & & \\
\hline PA & & & \multirow[t]{2}{*}{52} & \multirow[t]{2}{*}{0.22} & \multirow[t]{2}{*}{ DI water } & \multirow[t]{2}{*}{$1 \mathrm{M} \mathrm{NaCl}$} & \multirow[t]{2}{*}{$15 \mathrm{~mL} / \mathrm{min}$} & & $\mathrm{B}=0.125 \mathrm{LMH}$ & \multirow[t]{2}{*}{ [132] } \\
\hline Outer surface & & & & & & & & & $\begin{array}{c}\mathrm{S}=334.1 \mu \mathrm{m} \\
\mathrm{R}=85.1\end{array}$ & \\
\hline PES & \multirow{3}{*}{30.2} & \multirow{3}{*}{0.13} & & & \multirow{3}{*}{ DI water } & \multirow{3}{*}{$1 \mathrm{M} \mathrm{NaCl}$} & & & $\mathrm{A}=2.26 \mathrm{LMH} / \mathrm{bar}$ & \multirow{3}{*}{ [126] } \\
\hline PA & & & & & & & & & $\mathrm{B}=0.28 \mathrm{LMH}$ & \\
\hline outer surface & & & & & & & & & $\begin{array}{c}S=190 \mu \mathrm{m} \\
\mathrm{R}=85.1\end{array}$ & \\
\hline
\end{tabular}




\subsection{Issues and implications of TFC-A membranes}

Fig. 16a illustrates specific salt flux $\left(J_{S} / J_{v}\right)$ versus water flux/ $\Delta \pi\left(J_{v} / \Delta \pi\right)$ as a summary of data reported in Table 1 and 3. In the figure, the red symbols show the performance of TFC-A membrane with nanofiber substrate fabricated via electro-spinning method. This type of membranes show more reasonable water flux and specific reverse salt flux in comparison to the phase inversion membranes, including both flat sheet and hollow fiber. As stated in section 2.1, although the mechanical strength of nanofiber layer is not high, it does not seem to be an important issue since FO is not a pressure driven membrane process. However, it may still become an important issue even for FO process for its large-scale and long-term operation. One way of increasing the mechanical strength is the support by a backing fabric. Unfortunately, however, the water flux could be compromised by adding a support layer [100]. Therefore, to find the methods to increase the mechanical strength of nanofiber substrate is recommended as a future research focus. It is evident that hollow fiber TFC membranes present better performance compared with flat sheet membranes, however their fouling propensity should be further investigated. The number of studies where the fabrication of hollow fiber membranes having active layers at the outer surface of the membrane is very limited. Nonetheless, because these membranes have less tendency toward fouling, they are more suitable for wastewater treatment than the inner skin. The majority of the hollow fiber membranes synthesized for the FO process are with a PES substrate. Although PES possesses good mechanical and chemical properties, its fouling resistance is low. From this aspect polymers other than PES with lower fouling tendency should be searched for to prepare the substrate [66]. Surface modification, such as by PDA coating of the flat sheet membrane prepared by the phase inversion method, showed a reasonable performance [92]. It should be emphasized that the morphology of substrate skin layer can affect the active layer during the IP step, resulting in unpredictable membrane performances [135]; however, this aspect needs further investigation. 


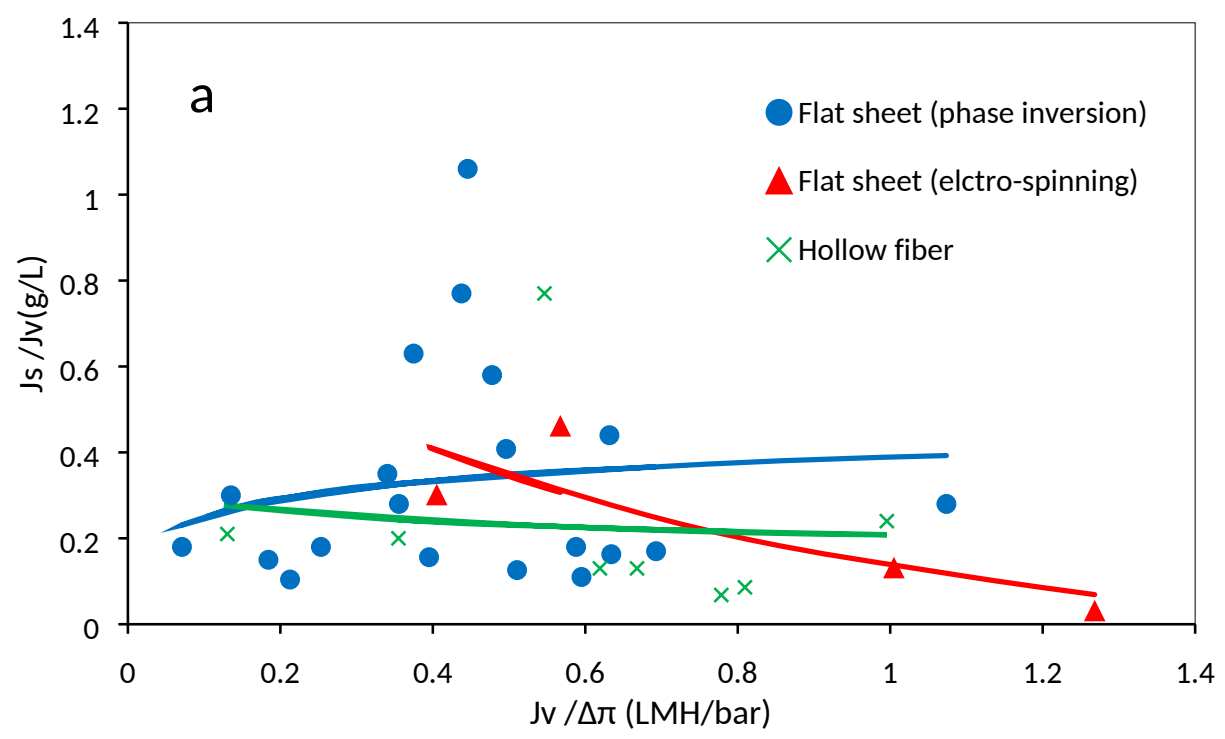

1

2

3
4

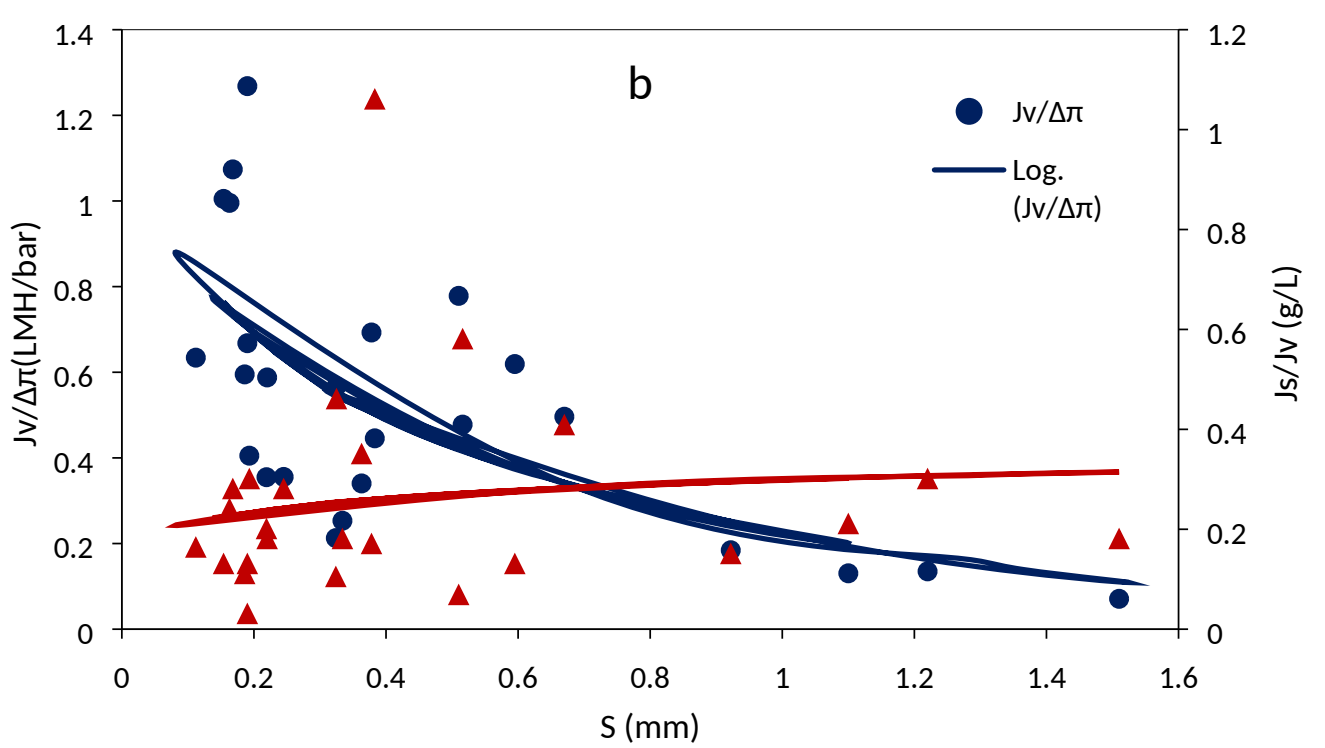

Fig 16: Water flux/driving force versus specific reverse a) specific reverse salt flux for and b) salt flux respectively for TFC-A FO membranes (the data point are taken from Tables 1 and 3)

Fig. 16b shows water flux $/ \Delta \pi\left(J_{v} / \Delta \pi\right)$ and specific reverse salt $\left(J_{S} / J_{v}\right)$ versus structural parameter. As evidenced, the structural parameter is an indication of internal concentration polarization [89]. For membrane processes with osmotic driving force, the lower the structural parameter, the higher the membrane performance $[100,136,137]$. It is because by increasing the structural parameter, 
the effective driving force across the membrane decreases. According to the theory, as shown in Figure 16b, with increasing $\mathrm{S}$, the trend of water flux changes is descending. However, the trend of specific reverse salt versus structural parameter is almost constant. The stability of these changes reveals a linear dependency of the salt permeability and water permeability coefficients. Because the separation properties of the active layer meaningfully depend on the properties of the sublayer, further investigation should be done to disclose the details.

\subsection{Performance of TFC-N membranes}

The addition of nanoparticles to substrate as well as to active layer of TFC membranes is one of the recent developments in this field that effectively changes the properties of resulted membrane. This technique affects the thickness, porosity, permeability, hydrophilicity, and roughness of substrate surface. It also influences the structure of active layer. Addition of nanomaterials in either aqueous or organic solution creates nano-scale pores in the polyamide active layer. Therefore, the addition of nanoparticles usually increases water flux but decreases the salt rejection [69, 138]. In addition, some nanoparticles have antifouling properties [40, 139-142]. In fact, addition of nanoparticles into substrate or active layer of a membrane can be considered as a kind of modification. The membrane modification approaches can be classified into two types, i.e., physical modification [143] and chemical modification [144]. Physical modification means that there is no chemical reaction among the components however in chemical modification chemical reaction occurs [65]. Lau et al. [145] reviewed the literature on making TFC-N membranes for different membrane processes. They identified several challenges in the fabrication of TFC-N membranes. Resolving these challenges can improve TFC-N membranes and make them suitable for a wide range of industrial applications. The main challenges are i) agglomeration of nanoparticles, due to their high surface energy, in the polyamide active layer, which reduces the surface area of nanoparticles as well as creates defects in the active layer. ii) loss of hydrophilic nanoparticles added to the aqueous solution at the time of removing the extra aqueous solution from the substrate surface. It would be better first to surface modify the hydrophilic nanoparticles and then add to the organic phase. iii) lack of chemical interaction between the polyamide active layer and nanoparticles. There are some solutions to these challenges, however, more studies are required to develop TFC-N membranes for industrial applications. In this section TFC-N membranes specified for FO process are reviewed in three classification as already depicted in Fig 1. In Fig. 17 schematic of different structure of TFC-N with their advantages and challenges are depicted: 
(a)

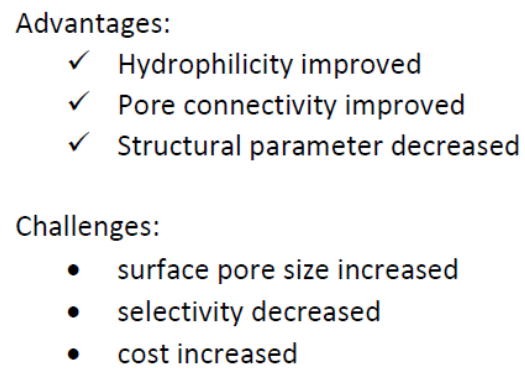

- $\quad$ surface pore size increased

- selectivity decreased

- cost increased

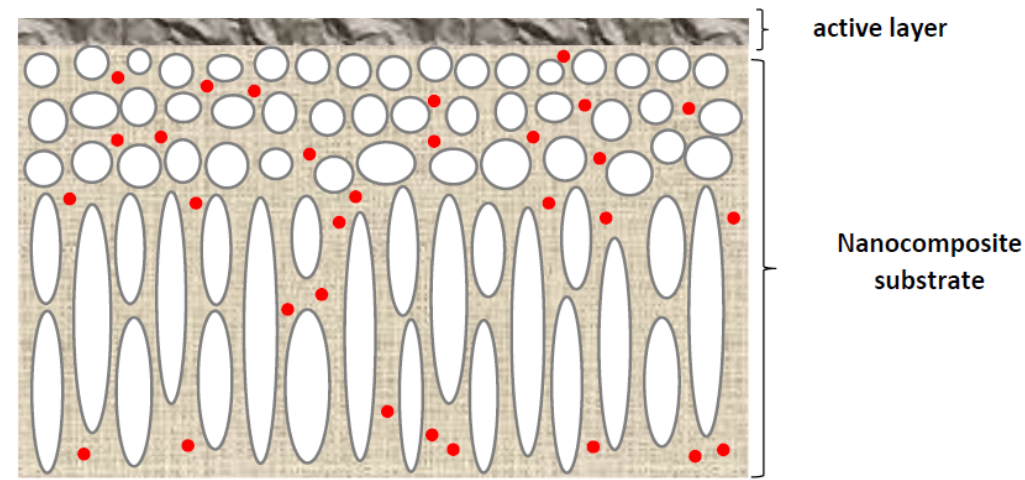

(b)

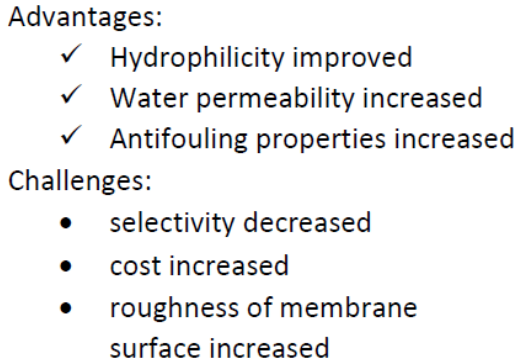

$\checkmark$ Hydrophilicity improved

$\checkmark$ Water permeability increased

$\checkmark$ Antifouling properties increased Challenges:

- selectivity decreased

- cost increased

- roughness of membrane surface increased

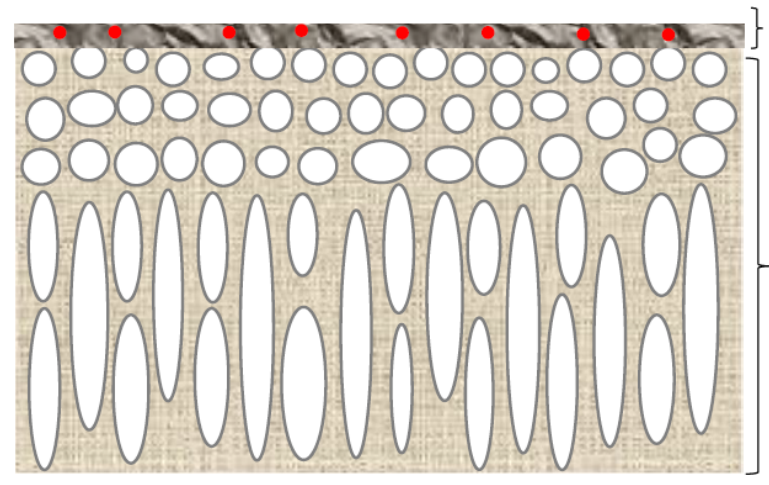

Nanocomposite active layer

substrate

(c)

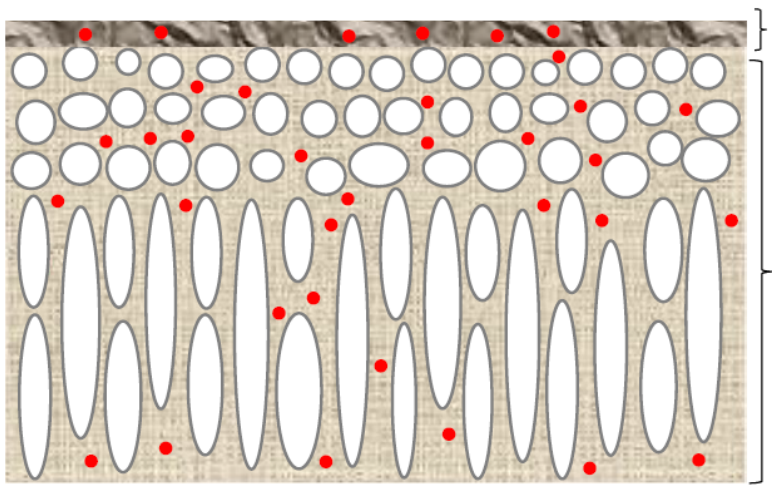

Nanocomposite active layer

Nanocomposite substrate

2 Fig 17: Schematic illustrations of different structures of TFC-N membranes: a) TFC-N with modified substrate, b) 3 TFC-N with modified active layer and c) TFC-N with modified both substrate and active layer 


\subsubsection{TFC-N with modified substrate}

\section{a) $\mathbf{T i O}_{2}$ nanoparticle in substrate}

3 Emadzadeh et al. [143] added titanium oxide $\left(\mathrm{TiO}_{2}\right)$ nanoparticles, in a range of 0-1 \%, to PSU

4 substrate which was coated with in-situ polymerized polyamide. By addition of the hydrophilic

$5 \mathrm{TiO}_{2}$ nanoparticles to the substrate forming a nano-composite sublayer, its hydrophilicity

6 increased. In Fig. 18, cross-sectional and surface SEM images are shown. From the figure, larger

7 finger-like pores were formed as well as the overall porosity increased upon addition of $\mathrm{TiO}_{2}$ due

8 to the increase in the water transfer rate from the coagulant to the polymer film. Further increase

9 in $\mathrm{TiO}_{2}$ concentration led to increase the nanoparticle agglomerates, observed as white spots. It

10 made the membrane surface rougher and the active layer more defective. As a result of these

11 morphological changes, water flux kept increasing while the salt rejection kept decreasing with an

12 increase in $\mathrm{TiO}_{2}$ addition. When the $\mathrm{TiO}_{2}$ concentration was higher than $0.5 \mathrm{wt} . \%$ reverse salt flux

13 became excessive due to the excessive nanoparticle agglomeration and defect formation. Hence, 14 it was concluded that $0.5 \mathrm{wt} . \%$ was the optimal value. 

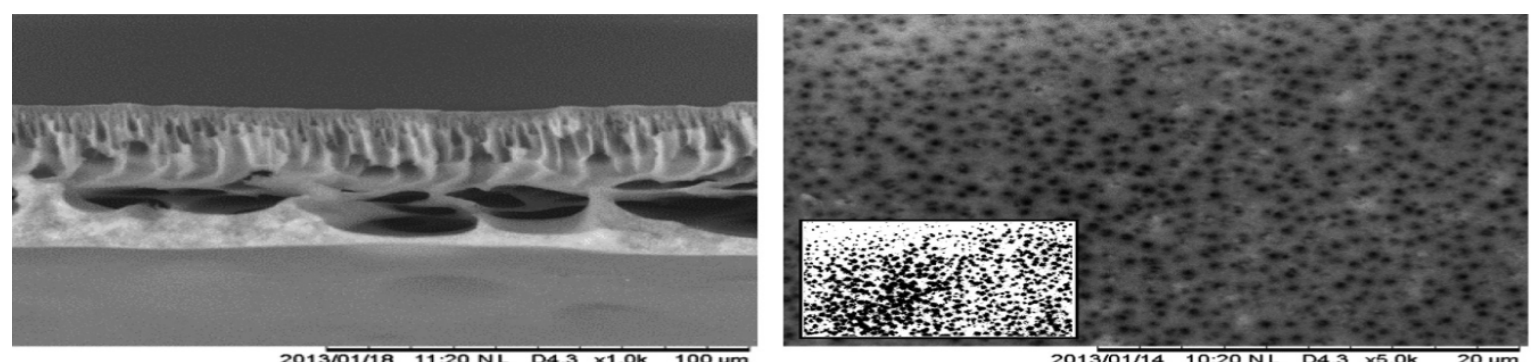

(a)
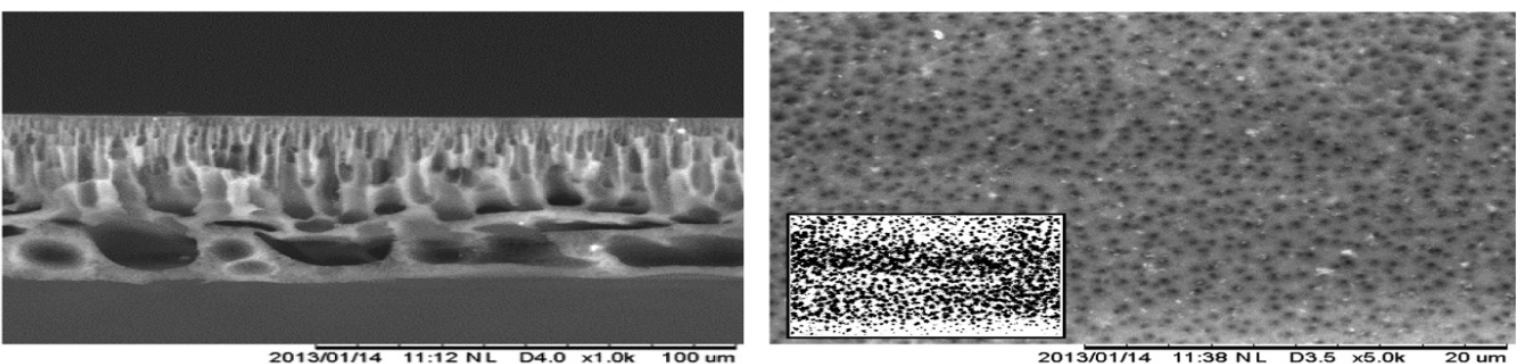

(b)
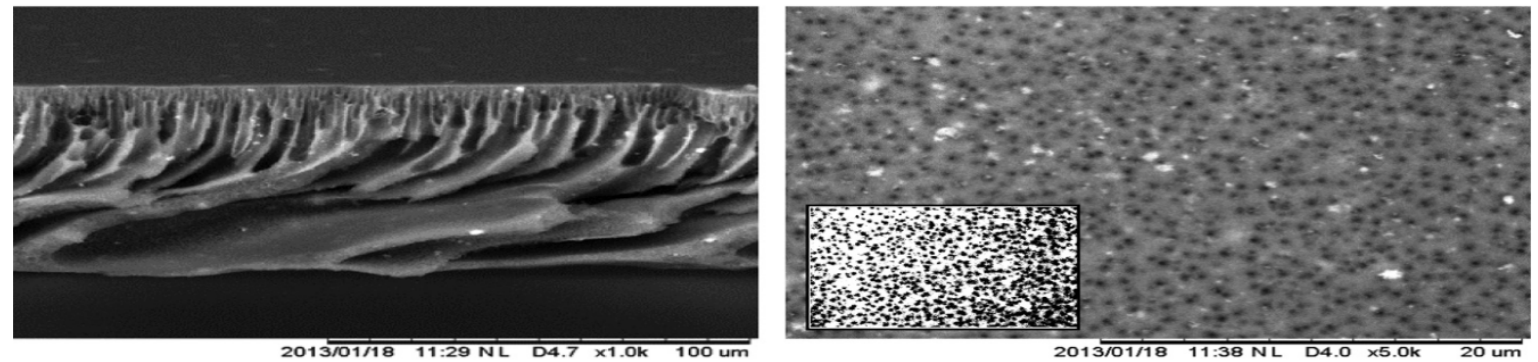

(C)
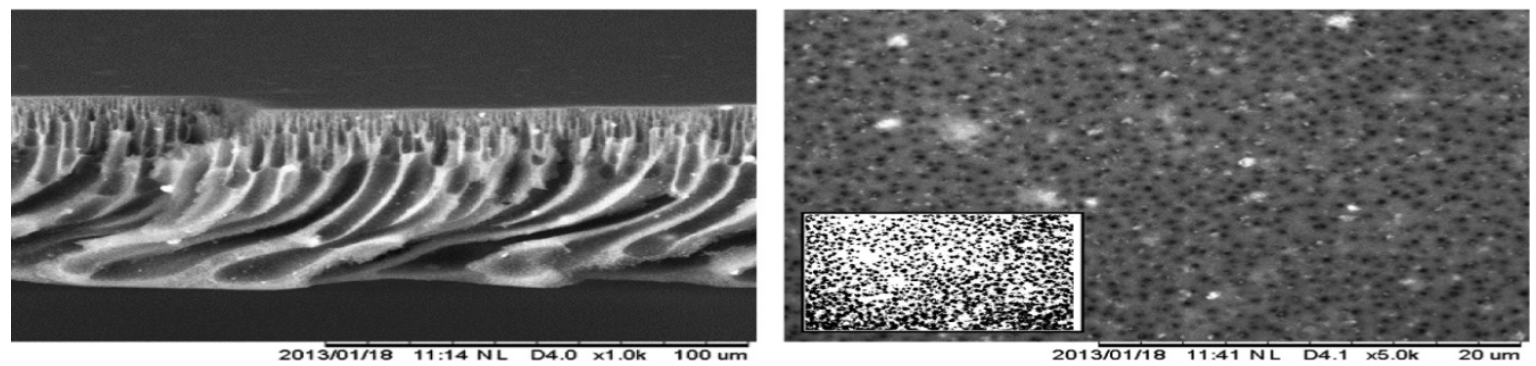

(d)

Fig 18: SEM of the cross-section and top surface of PSF substrates prepared from different nanoparticles loadings, (a) substrate (control), (b) substrate 0.5 , (c) substrate 0.75 and (d) substrate1.0 (note: threshold image placed on bottom left corner of each top surface was used to determine average pore size using ImageJ software) [143]

\section{b) ZnO nanoparticle in substrate}

Rastgar et al. [146] studied the effect of addition of two nanoparticles to PES substrate on the performance of a polyamide TFC membrane. The two applied nanoparticles were Zinc oxide nanoparticles (ZNPs) and $\mathrm{ZnO}^{-\mathrm{SiO}_{2}}$ core-shell nanoparticles (ZSCSNPs). ZNPs were prepared by

10 sol-gel method and then covered with hydrophilic silica $\left(\mathrm{SiO}_{2}\right)$ to synthesize ZSCSNPs which were 11 more hydrophilic than ZNPs. Table 4 shows the composition of the dope solution used to fabricate 
1 the membranes. The SEM images showed that the addition of nanoparticles to the substrate led to 2 the formation of a more finger-like structure and an increase in the total porosity. These changes 3 were due to the increase of water transfer from the coagulation to the polymeric film.

5 Table 4: The compositions of dope solution used to fabricate the substrates [146]

\begin{tabular}{lccccc}
\hline support layer label & $\begin{array}{c}\text { PES } \\
(\mathrm{wt} \%)\end{array}$ & $\begin{array}{c}\text { PEG-200 } \\
(\mathrm{wt} \%)\end{array}$ & $\begin{array}{c}\text { NMP } \\
(\mathrm{wt} \%)\end{array}$ & $\begin{array}{c}\text { Nanomaterial } \\
(\mathrm{type})\end{array}$ & Weight content \\
\hline PES & 18.00 & 10.00 & 72.00 & - & 0.00 \\
\hline PES-ZNP-1 & 18.00 & 10.00 & 72.00 & ZNPs & 1.00 \\
\hline PES-ZSCSNP-0.1 & 18.00 & 10.00 & 72.00 & ZSCSNPs & 0.10 \\
\hline PES-ZSCSNP-0.5 & 18.00 & 10.00 & 72.00 & ZSCSNPs & 0.50 \\
\hline PES-ZSCSNP-1 & 18.00 & 10.00 & 72.00 & ZSCSNPs & 1.00 \\
\hline PES-ZSCSNP-2 & 18.00 & 10.00 & 72.00 & ZSCSNPs & 2.00 \\
\hline
\end{tabular}

8 Fig. 19 shows the AFM surface images and the roughness parameters of the neat PES and the 9 nanocomposite substrates for their TFC-N membranes. As can be seen, the addition of the 10 nanoparticles increased the surface roughness, likely due to the nanoparticle agglomeration, Both 11 ZNP and ZSCSNP had the same effect but the effect of ZNP was stronger. Interestingly, the salt 12 rejection was almost the same after the addition of either of the two nanoparticles. It is presumably 13 due to the negligible difference in the size of the utilized nano-particles, i.e. 30 and $50 \mathrm{~nm}$. Despite 14 the higher roughness of ZNP added substrate, water flux of ZSCSNPs TFC-N membrane was 15 higher. It was most likely due to the higher hydrophilicity of ZSCSNP [146]. 

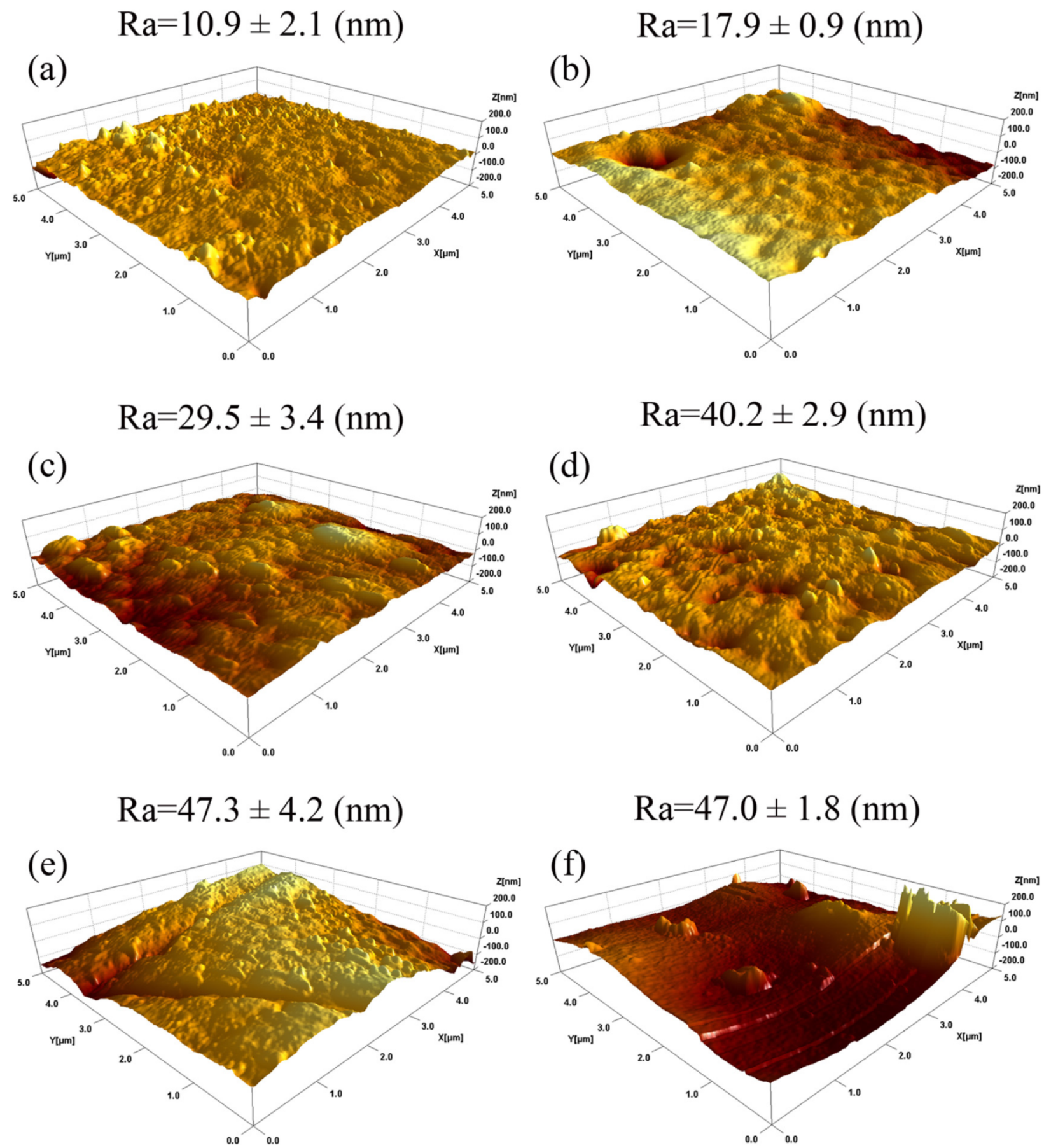

Fig 19: AFM images of PES-TFC-N (a), PES-ZSCSNP-0.1 (b), PES-ZSCSNP-0.5 (c), PES-ZSCSNP-1 (d), PESZSCSNP-2 (e) and PES-ZNP-1 (f) substrate layers. [146]

\section{c) Silica nanoparticle in substrate}

6 Tian et al. [147] incorporated silica nanoparticles in the TFC-N electro-spun polyetherimide (PEI) 7 nanofiber substrate to reduce the ICP in FO. In comparison with the finger-like structure formed 8 by the phase inversion method, the inter-connected macropores of nanofibrous membranes have 
1 the potential to decrease the membrane structural parameter, with lower tortuosity coefficient and

2 higher porosity. The reason for the addition of silica nanoparticles was to maintain the porosity 3 during the heat treatment of nanofibers either by heat-press. The outputs revealed high thermal 4 capability of the nanofibers synthesized based on silica nanoparticles during the heat-press 5 treatment.

Ma et al. [148] studied the effect of addition of zeolite nanoparticles into the polyamide active layer on the performance of TFC-N membranes in FO. PSF was used to make the substrate, and zeolite nanoparticles were added to the organic phase in interfacial polymerization. The surface morphology, roughness and contact angle all changed by the addition of zeolite nanoparticles. These changes finally affected the separation properties of the fabricated membranes. The addition of zeolite nanoparticles into the polyamide selective layer of FO membrane increased the FO

\section{b) Amino-functionalized titanate nanotubes in active layer}

Emadzadeh et al. [144] used Amino-functionalized titanate nanotubes $\left(\mathrm{NH}_{2}-\mathrm{TNTs}\right)$ in the organic phase to make TFC-N membrane for FO. Titanate nanotubes (TNTs) were surface modified by reacting with $\mathrm{N}$-(2-Aminoethyl)-3-aminopropyltrimethoxysilane (AAPTS) to form $\mathrm{NH}_{2}$-TNTs, which was confirmed by ATR-FTIR. Also, crystalline structure and tubular morphology of $\mathrm{NH}_{2}$ TNTs was characterized, by using XRD and TEM tests respectively. Nanoparticle $\mathrm{NH}_{2}$-TNTs bind chemically with the polyamide active layer. In Fig. 20, the bond between the polyamide and nanoparticle $\mathrm{NH}_{2}$-TNTs is shown. 


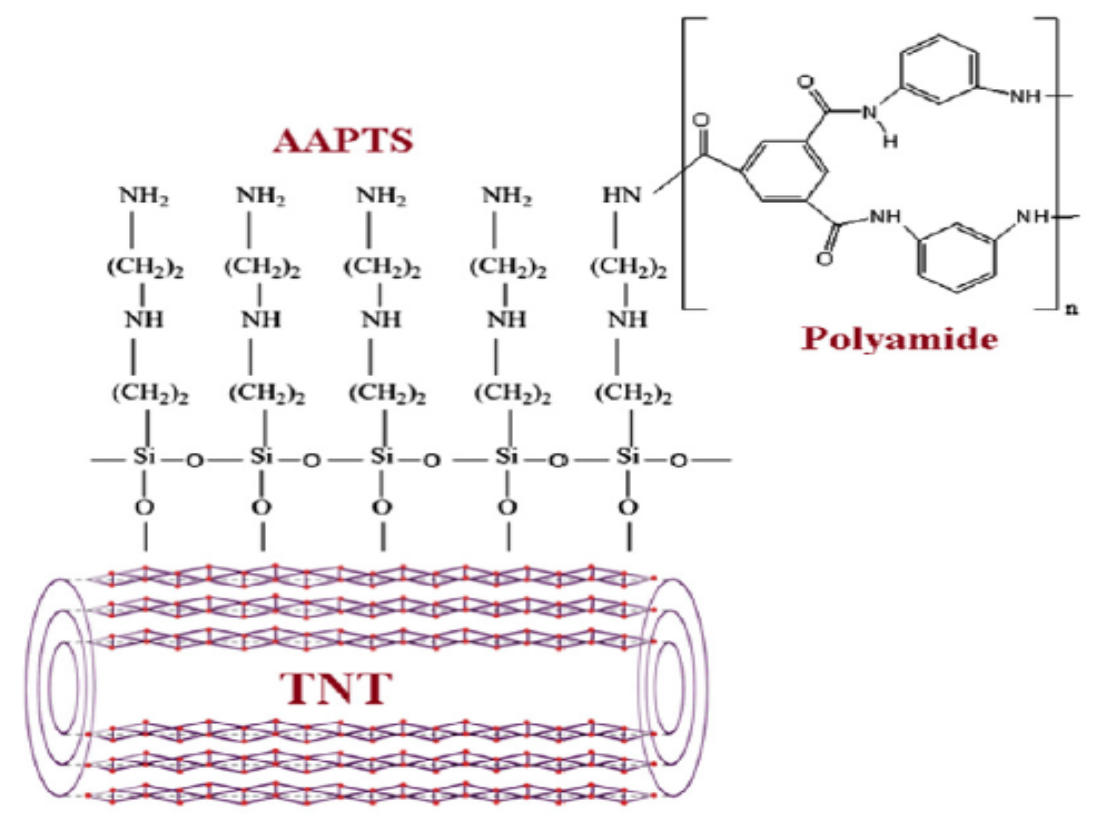

\section{Interaction between functionalized} TNTs and polyamide

Fig 20: Schematic illustration of interaction between polyamide (PA) and $\mathrm{NH}_{2}$-TNTs [144]

Fig. 21 shows the water flux and salt rejection as a function of $\mathrm{NH}_{2}$-TNTs content. Upon nanoparticle addition, water flux increased and it kept increasing with an increase in the nanoparticle concentration. The increase in water flux was caused by i) increase in hydrophilicity of the membrane, ii) increase in "leaf-like" and ridge and valley structure, iii) increase in the water current through the cavity between the nanoparticles and polymer matrix and iv) increase in the water current through the hollow nanoparticles. The increase of nanoparticle concentration up to $0.05 \mathrm{wt} . \%$ exhibited favorable effects such as increase in aqueous and organic phase compatibility and increase of the polyamide crosslinking via $-\mathrm{NH}_{2}$ groups attached to the nanoparticles in the active layer, resulted in higher salt rejection. However, further increase in nanoparticles concentration started to decrease the cross-linking and conversely increased the voids between the $\mathrm{NH}_{2}$-TNTs and polyamide matrix. As a result, salt rejection decreased [144]. This also was observed by the other researchers [149]. 


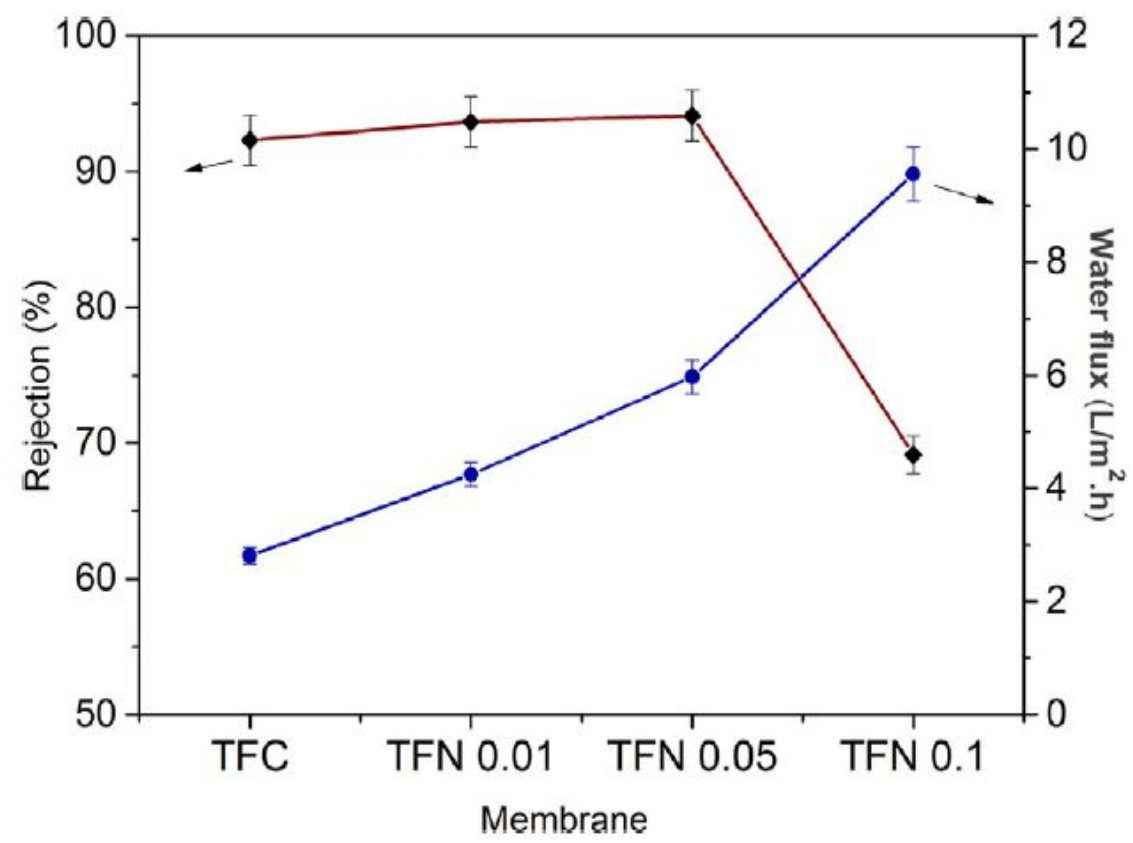

1

Fig 21: Water flux and $\mathrm{NaCl}$ rejection of TFC/TFC-N membranes (Test conditions: 2.5 bar, $25^{\circ} \mathrm{C}$ and $20 \mathrm{mM} \mathrm{NaCl}$ aqueous solution) [144]

\section{c) GOAg nanoparticles in active layer}

Faria et al. [139] used an antibacterial nanoparticle to make a TFC-N membrane. The nanoparticles (GOAg) were made of graphene oxide (GO) and silver nanoparticles (Ag). Also, a polyamide TFC membrane of HTI was used in this research. The carboxyl groups on the GOAg nanoparticle formed covalent bonds with the amine functional group on ethylene diamine (ED) functionalized TFC membrane. The nanoparticle showed antibacterial and antifouling effects on the membrane without changing its inherent properties. By addition of the nanoparticle, the flux reduction due to the membrane fouling decreased by $30 \%$.

\section{d) MOF in active layer}

Zirehpour et al.[150] synthesized nano-sized metal-organic framework (MOF) particles consisting of silver (I) and 1,3,5-benzene tricarboxylic acid. MOF was incorporated into the polyamide layer of membranes to improve the structure of TFC membrane. A good compatibility between the MOF and the aromatic polyamide layer was reported. This nano particle enhanced the hydrophilicity and transport properties of the active layer without any impact on selectivity. They also monitored the performance stability of the membrane in FO seawater desalination in the course of time. The Caspian seawater and $2 \mathrm{M} \mathrm{NaCl}$ was used as feed and draw solution respectively (Fig. 22). The FO seawater desalination flux through the TFC-N membrane was very stable throughout the testing interval (only about 7\% flux decline), while the normal TFC membrane presented about $18 \%$ reduction in water flux. The reductions in FO water flux are attributed mostly to the descent 
in driving force of FO process and membrane fouling. According to the vast variety of MOFs with different metal ions or clusters coordinated to different organic ligands to form one, two, or threedimensional structures, it is believed that the populated family of MOF needs more focus as an attractive research area in TFC-N membranes.

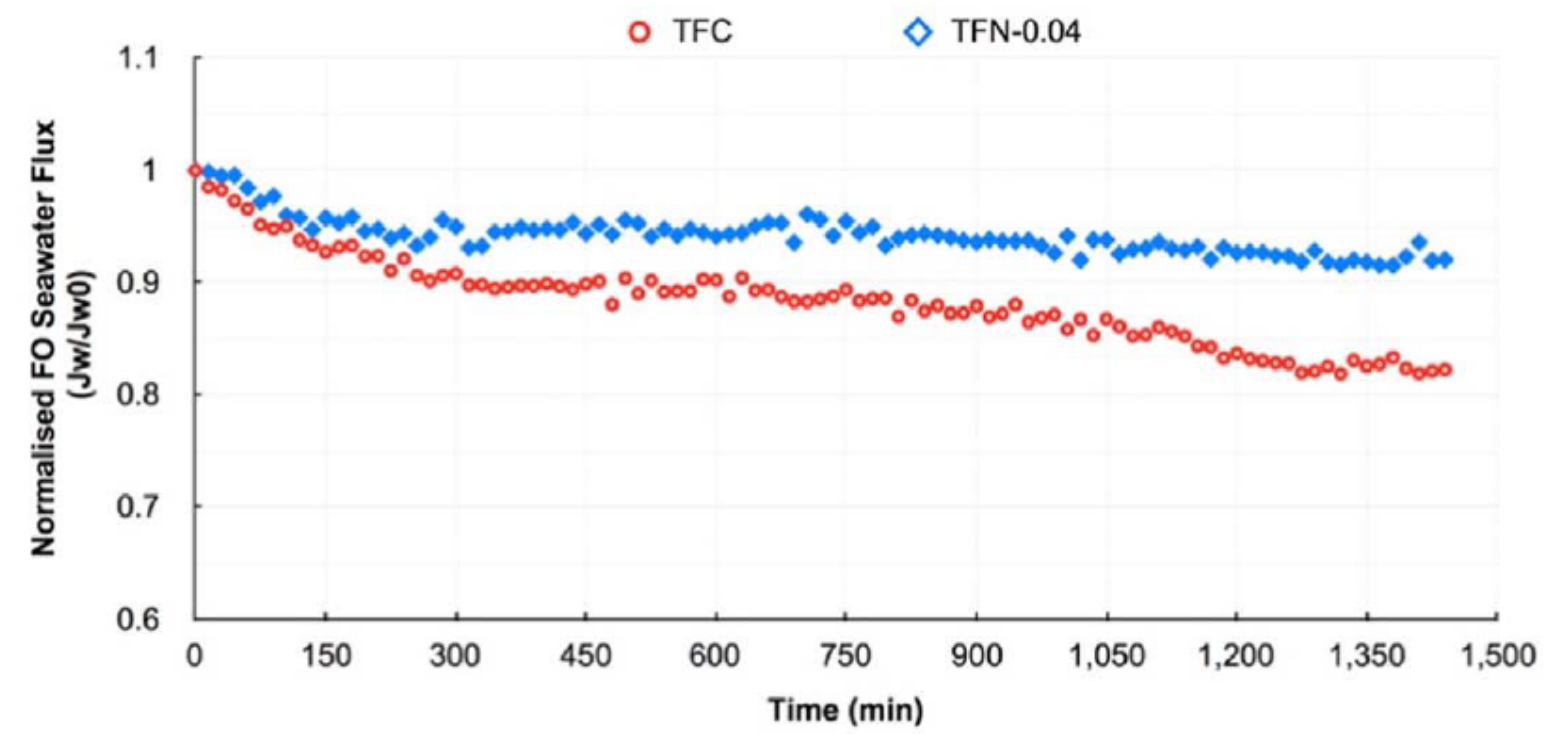

Fig 22: normalized (JW/Jw0) FO seawater flux decline of the membranes over time ( $\left.\mathrm{T}=25^{\circ} \mathrm{C}, \mathrm{AL}-\mathrm{FS}\right)[150]$

\subsubsection{TFC-N with modified both substrate and active layer concurrently}

Rezaei-DashtArzhandi et al. [149] fabricated TFC-N membranes with both substrate and PA active layer incorporated with Halloysite nanotubes (HNTs) and graphite-like carbon nitrite (g-C3N4), respectively. The contact angle measurement revealed that by adding g-C3N4 within the PA active layer super hydrophilic surface were formed. The FO water flux without increasing reverse solute flux was considerably enhanced by a combination of the positive features contributed by the substrate and the PA active layer modified by HNTs and g-C3N4 respectively. However, the results of this paper proved that the modification of the PA selective layer has presented dominant role towards improving FO membrane performances in comparison to the substrate modification. The antifouling property of TFC-N membranes can be attributed to the enhancement of surface hydrophilicity [151].

Also, Ohland et al.[152] used hydroxyapatite particles (Hapf) functionalized by plasma treatment as a nanoparticle additive into a porous cellulose acetate substrate and a selective polyamide layer concurrently. They concluded that the addition of the hydrophilic particles in porous CA substrate was able to enhance the hydrophilicity of the matrix and reduce ICP. Furthermore, Adding Hapf in the PA layer increased the affinity toward water and reorganized the polymer chains, resulting in lower diffusion resistance and enhancement of water permeability. 
1 Tables 5, 6 and 7 reviews the papers on the flux of water and the specific reverse salt flux of the 2 three types of TFC-N membranes respectively: TFC-N with modified substrate, TFC-N with 3 modified active layer and TFC-N with modified both substrate and active layer.

\section{Issues and implications of TFC-N membranes}

- Nanomaterials should be modified in order to become more compatible with substrate or active layer to prevent their loss during the process especially in the long term operation. It is suggested to examine the long-term operation of TFC-N membranes to evaluate the nanomaterial loss during the operation.

- Although the performance of FO membranes can be improved by applying a tiny amount of nanoparticles, nano-materials can be toxic and expensive. Therefore, nontoxic and costeffective nanomaterials should be used in an FO water treatment process. 
1 Table 5: A summary of the studies on TFC-N with modified substrate

\begin{tabular}{|c|c|c|c|c|c|c|c|c|c|c|c|}
\hline \multirow{2}{*}{\multicolumn{2}{|c|}{$\begin{array}{cc}\begin{array}{c}\text { Support } \\
\text { layer }\end{array} \\
\text { Active layer } \\
\end{array}$}} & \multirow[b]{3}{*}{ Structure } & \multicolumn{4}{|c|}{ Performance } & \multicolumn{3}{|c|}{ Experimental condition } & \multirow[b]{3}{*}{$\begin{array}{c}\text { Intrinsic } \\
\text { properties }\end{array}$} & \multirow[b]{3}{*}{ ref } \\
\hline & & & \multicolumn{2}{|c|}{ AL-FS(FO) } & \multicolumn{2}{|c|}{ AL-DS(PRO) } & \multirow[b]{2}{*}{$\begin{array}{c}\text { Feed } \\
\text { solution }\end{array}$} & \multirow[b]{2}{*}{$\begin{array}{r}\text { Draw } \\
\text { solution }\end{array}$} & \multirow[b]{2}{*}{$\begin{array}{c}\text { cross-flow } \\
\text { velocity }\end{array}$} & & \\
\hline & Nano material & & $\begin{array}{c}\mathrm{J}_{\mathrm{v}} \\
(\mathrm{LMH})\end{array}$ & $\begin{array}{l}\mathrm{J}_{\mathrm{S}} / \mathrm{J}_{\mathrm{v}} \\
(\mathrm{g} / \mathrm{L})\end{array}$ & $\begin{array}{c}\mathrm{J}_{\mathrm{v}} \\
(\mathrm{LMH})\end{array}$ & $\begin{array}{l}\mathrm{J}_{\mathrm{S}} / \mathrm{J}_{\mathrm{v}} \\
(\mathrm{g} / \mathrm{L})\end{array}$ & & & & & \\
\hline & $\begin{array}{c}\text { PSF } \\
\text { PA } \\
\text { Zeolite }\end{array}$ & Flat sheet & 40 & 0.7 & 86 & 0.66 & DI water & $\begin{array}{c}2 \mathrm{M} \\
\mathrm{NaCl}\end{array}$ & \multicolumn{3}{|c|}{$\begin{array}{c}\mathrm{A}=3.2 \mathrm{LMH} / \mathrm{bar} \\
\mathrm{S}=0.34 \mathrm{~mm} \\
\mathrm{R}=90.6\end{array}$} \\
\hline & $\begin{array}{l}\mathrm{PSF} \\
\mathrm{PA} \\
\mathrm{TiO}_{2}\end{array}$ & Flat sheet & 29.76 & 0.26 & 56.27 & 0.25 & $\begin{array}{c}10 \mathrm{mM} \\
\mathrm{NaCl}\end{array}$ & $\begin{array}{l}2 \mathrm{M} \\
\mathrm{NaCl}\end{array}$ & $\begin{array}{l}\mathrm{A}=1 \\
\mathrm{~B}=1\end{array}$ & $\begin{array}{l}=1.96 \mathrm{LMH} / \mathrm{bar} \\
=10.66 \times 10^{-8} \mathrm{~m} / \mathrm{s} \\
\mathrm{S}=.42 \mathrm{~mm} \\
\mathrm{R}=92.4\end{array}$ & [143] \\
\hline & $\begin{array}{l}\text { PSF } \\
\text { PA } \\
\text { GO }\end{array}$ & Flat sheet & 19.77 & 0.16 & 40.5 & 0.16 & DI water & $\begin{array}{r}0.5 \mathrm{M} \\
\mathrm{NaCl}\end{array}$ & $\begin{array}{r}\mathrm{A}=1 \\
\mathrm{~B}=\end{array}$ & $\begin{array}{c}=1.76 \mathrm{LMH} / \mathrm{bar} \\
\mathrm{B}=0.19 \mathrm{LMH} \\
\mathrm{S}=191 \mu \mathrm{m} \\
\mathrm{R}=98.71\end{array}$ & {$[154]$} \\
\hline & $\begin{array}{l}\text { PSF } \\
\text { PA } \\
\text { silica }\end{array}$ & Flat sheet & 31 & 0.24 & 60.5 & 0.26 & DI water & $\begin{array}{l}1 \mathrm{M} \\
\mathrm{NaCl}\end{array}$ & $\begin{array}{r}\mathrm{A}=1 \\
\mathrm{~B}=\end{array}$ & $\begin{array}{l}=1.64 \mathrm{LMH} / \mathrm{bar} \\
\mathrm{B}=0.29 \mathrm{LMH} \\
\mathrm{S}=169 \mu \mathrm{m}\end{array}$ & {$[155]$} \\
\hline & $\begin{array}{c}\text { polyetherimide } \\
\text { (PEI) } \\
\text { PA } \\
\text { carbon nanotubes } \\
\text { (CNTs) }\end{array}$ & Flat sheet & 32.8 & 0.12 & 61.3 & 0.07 & DI water & $\begin{array}{l}1 \mathrm{M} \\
\mathrm{NaCl}\end{array}$ & $\begin{array}{r}\mathrm{A}= \\
\mathrm{B}\end{array}$ & $\begin{array}{l}=2.6 \mathrm{LMH} / \mathrm{bar} \\
\mathrm{B}=0.7 \mathrm{LMH} \\
\mathrm{S}=210 \mu \mathrm{m}\end{array}$ & {$[156]$} \\
\hline $\begin{array}{l}\text { PSF } \\
\text { PA } \\
\text { HNTs }\end{array}$ & Flat sheet & 27.71 & 0.52 & 43.25 & 0.63 & $\begin{array}{c}10 \mathrm{mM} \\
\mathrm{NaCl}\end{array}$ & $\begin{array}{l}2 \mathrm{M} \\
\mathrm{NaCl}\end{array}$ & $350 \mathrm{ml} / \mathrm{min}$ & $\begin{array}{c}\mathrm{A}=2 \mathrm{LMH} / \mathrm{bar} \\
\mathrm{B}=9.43 \times 10^{-8} \mathrm{~m} / \mathrm{s} \\
\mathrm{S}=0.37 \mathrm{~mm}\end{array}$ & [157] & \\
\hline $\begin{array}{r}\text { PSF } \\
\text { PA } \\
\text { Layered doub } \\
\text { hydroxide (LI }\end{array}$ & Flat sheet & 18.1 & 0.44 & 34.6 & 0.36 & DI water & $\begin{array}{c}1 \mathrm{M} \\
\mathrm{NaCl}\end{array}$ & $190 \mathrm{ml} / \mathrm{min}$ & $\mathrm{S}=57.4 \mu \mathrm{m}$ & {$[158]$} & \\
\hline
\end{tabular}

2 
2 Table 5 (continued)

\begin{tabular}{|c|c|c|c|c|c|c|c|c|c|c|}
\hline \multirow{2}{*}{$\begin{array}{c}\begin{array}{c}\text { Support } \\
\text { layer }\end{array} \\
\text { Active layer } \\
\end{array}$} & \multirow[b]{3}{*}{ structure } & \multicolumn{4}{|c|}{ performance } & \multicolumn{3}{|c|}{ Experimental condition } & \multirow[b]{3}{*}{$\begin{array}{c}\text { Intrinsic } \\
\text { properties }\end{array}$} & \multirow[b]{3}{*}{ ref } \\
\hline & & \multicolumn{2}{|c|}{ AL-FS(FO) } & \multicolumn{2}{|c|}{ AL-DS(PRO) } & \multirow[b]{2}{*}{$\begin{array}{c}\text { Feed } \\
\text { solution }\end{array}$} & \multirow[b]{2}{*}{$\begin{array}{r}\text { Draw } \\
\text { solution }\end{array}$} & \multirow[b]{2}{*}{$\begin{array}{c}\text { cross-flow } \\
\text { velocity }\end{array}$} & & \\
\hline Nano material & & $\begin{array}{c}\mathrm{J}_{\mathrm{v}} \\
(\mathrm{LMH})\end{array}$ & $\begin{array}{l}J_{\mathrm{S}} / \mathrm{J}_{\mathrm{v}} \\
(\mathrm{g} / \mathrm{L})\end{array}$ & $\begin{array}{c}\mathrm{J}_{\mathrm{v}} \\
(\mathrm{LMH})\end{array}$ & $\begin{array}{l}\mathrm{J}_{\mathrm{s}} / \mathrm{J}_{\mathrm{v}} \\
(\mathrm{g} / \mathrm{L})\end{array}$ & & & & & \\
\hline $\begin{array}{c}\text { PSF } \\
\text { PA } \\
\text { LDH/GO }\end{array}$ & Flat sheet & 13.4 & 0.46 & 23.6 & 0.29 & DI water & $\begin{array}{c}1 \mathrm{M} \\
\mathrm{NaCl}\end{array}$ & $190 \mathrm{ml} / \mathrm{min}$ & $\begin{array}{c}\mathrm{A}=0.53 \mathrm{LMH} / \mathrm{bar} \\
\mathrm{B}=0.15 \mathrm{LMH} \\
\mathrm{S}=138 \mu \mathrm{m}\end{array}$ & {$[159]$} \\
\hline $\begin{array}{c}\text { PES } \\
\mathrm{PA} \\
\mathrm{ZnO}\end{array}$ & Flat sheet & 31 & 0.41 & 43 & 0.42 & DI water & $\begin{array}{c}1 \mathrm{M} \\
\mathrm{NaCl}\end{array}$ & $8.3 \mathrm{~cm} / \mathrm{s}$ & $\begin{array}{c}\mathrm{A}=3.12 \mathrm{LMH} / \mathrm{bar} \\
\mathrm{B}=3.75 \mathrm{LMH} \\
\mathrm{S}=300 \mu \mathrm{m} \\
\mathrm{R}=77.94\end{array}$ & {$[146]$} \\
\hline $\begin{array}{c}\text { PES } \\
\text { PA } \\
\text { ZSCSNPs }\end{array}$ & Flat sheet & 33.5 & 0.36 & 50.1 & 0.36 & DI water & $\begin{array}{c}1 \mathrm{M} \\
\mathrm{NaCl}\end{array}$ & $8.3 \mathrm{~cm} / \mathrm{s}$ & $\begin{array}{c}\mathrm{A}=3.47 \mathrm{LMH} / \mathrm{bar} \\
\mathrm{B}=4.01 \mathrm{LMH} \\
\mathrm{S}=297 \mu \mathrm{m} \\
\mathrm{R}=78.60\end{array}$ & {$[146]$} \\
\hline $\begin{array}{c}\mathrm{PEI} \\
\mathrm{PA} \\
\mathrm{SiO}_{2}\end{array}$ & Flat sheet & 42 & 0.12 & 72 & 0.1 & DI water & $\begin{array}{l}1 \mathrm{M} \\
\mathrm{NaCl}\end{array}$ & $9 \mathrm{~cm} / \mathrm{s}$ & $\begin{array}{c}\mathrm{A}=2.99 \mathrm{LMH} / \mathrm{bar} \\
\mathrm{B}=0.41 \mathrm{LMH} \\
\mathrm{S}=174 \mu \mathrm{m} \\
\mathrm{R}=74.2\end{array}$ & {$[147]$} \\
\hline $\begin{array}{l}\text { PSF } \\
\text { PA } \\
\text { GO }\end{array}$ & $\begin{array}{c}\text { Dual- } \\
\text { layered } \\
\text { Flat sheet }\end{array}$ & 33.8 & 0.19 & 61.5 & 0.18 & DI water & $\begin{array}{c}1 \mathrm{M} \\
\mathrm{NaCl}\end{array}$ & $16.7 \mathrm{~cm} / \mathrm{s}$ & $\begin{array}{c}\mathrm{A}=1.46 \mathrm{LMH} / \mathrm{bar} \\
\mathrm{B}=0.25 \mathrm{LMH} \\
\mathrm{S}=130 \mu \mathrm{m}\end{array}$ & {$[160]$} \\
\hline $\begin{array}{c}\text { PSF } \\
\text { PA } \\
\text { Imogolite } \\
\text { nanotubes (INTs) }\end{array}$ & Flat sheet & 7.17 & 1.47 & 9.5 & 2.6 & DI water & $\begin{array}{l}1 \mathrm{M} \\
\mathrm{NaCl}\end{array}$ & $\begin{array}{c}333.3 \\
\mathrm{ml} / \mathrm{min}\end{array}$ & $\begin{array}{c}\mathrm{A}=3.03 \mathrm{LMH} / \mathrm{bar} \\
\mathrm{B}=2.92 \mathrm{LMH} \\
\mathrm{S}=2.09 \mathrm{~mm} \\
\mathrm{R}=83\end{array}$ & {$[161]$} \\
\hline $\begin{array}{c}\text { PSF } \\
\text { PA } \\
\text { MOF (UiO-66) }\end{array}$ & Flat sheet & 24.5 & 0.18 & 39.4 & & DI water & $\begin{array}{l}1 \mathrm{M} \\
\mathrm{NaCl}\end{array}$ & $1.1 \mathrm{~cm} / \mathrm{s}$ & $\begin{array}{c}\mathrm{A}=3.31 \mathrm{LMH} / \mathrm{bar} \\
\mathrm{B}=0.53 \mathrm{LMH} \\
\mathrm{S}=351 \mu \mathrm{m} \\
\mathrm{R}=92.6\end{array}$ & {$[162]$} \\
\hline
\end{tabular}


1 Table 6: A summary of the studies on TFC-N with modified active layer

\begin{tabular}{|c|c|c|c|c|c|c|c|c|c|c|}
\hline \multirow{2}{*}{$\begin{array}{c}\begin{array}{c}\text { Support } \\
\text { layer }\end{array} \\
\text { Active layer }\end{array}$} & \multirow[b]{3}{*}{ structure } & \multicolumn{4}{|c|}{ performance } & \multicolumn{3}{|c|}{ Experimental condition } & \multirow[b]{3}{*}{$\begin{array}{l}\text { Intrinsic } \\
\text { properties }\end{array}$} & \multirow[b]{3}{*}{ ref } \\
\hline & & \multicolumn{2}{|c|}{ AL-FS(FO) } & \multicolumn{2}{|c|}{ AL-DS(PRO) } & \multirow[b]{2}{*}{$\begin{array}{c}\text { Feed } \\
\text { solution }\end{array}$} & \multirow[b]{2}{*}{$\begin{array}{r}\text { Draw } \\
\text { solution }\end{array}$} & \multirow[b]{2}{*}{$\begin{array}{l}\text { cross-flow } \\
\text { velocity }\end{array}$} & & \\
\hline Nano material & & $\begin{array}{c}\mathrm{J}_{\mathrm{v}} \\
(\mathrm{LMH})\end{array}$ & $\begin{array}{l}\mathrm{J}_{\mathrm{s}} / \mathrm{J}_{\mathrm{v}} \\
(\mathrm{g} / \mathrm{L})\end{array}$ & $\begin{array}{c}\mathrm{J}_{\mathrm{v}} \\
(\mathrm{LMH})\end{array}$ & $\begin{array}{l}\mathrm{J}_{\mathrm{s}} / \mathrm{J}_{\mathrm{v}} \\
(\mathrm{g} / \mathrm{L})\end{array}$ & & & & & \\
\hline PSF & & & & & & & & & $\mathrm{A}=7.15 \times 10^{-12} \mathrm{~m} / \mathrm{s} \mathrm{pa}$ & \\
\hline $\begin{array}{c}\text { PA } \\
\text { Zeolite }\end{array}$ & Flat sheet & 17.4 & 0.45 & 38.2 & 0.37 & DI water & $1 \mathrm{M} \mathrm{NaCl}$ & $500 \mathrm{~mL} / \mathrm{min}$ & $\begin{array}{c}\mathrm{B}=43.7 \times 10^{-8} \mathrm{~m} / \mathrm{s} \\
\mathrm{R}=77.6\end{array}$ & [148] \\
\hline $\begin{array}{c}\mathrm{PSF} \\
\mathrm{PA} \\
\mathrm{SiO} 2\end{array}$ & Flat sheet & 15 & 0.1 & 25 & 0.14 & $\begin{array}{c}10 \mathrm{mM} \\
\mathrm{NaCl}\end{array}$ & $2 \mathrm{M} \mathrm{NaCl}$ & $800 \mathrm{~mL} / \mathrm{min}$ & $\begin{array}{c}\mathrm{A}=9.52 \times 10^{-12} \mathrm{~m} / \mathrm{s} \mathrm{pa} \\
\mathrm{B}=28.4 \times 10^{-8} \mathrm{~m} / \mathrm{s} \\
\mathrm{S}=0.368 \mathrm{~mm} \\
\mathrm{R}=89\end{array}$ & [138] \\
\hline $\begin{array}{c}\mathrm{PSF} \\
\mathrm{PA} \\
\mathrm{NH}_{2}-\mathrm{TNTs} \\
\end{array}$ & Flat sheet & 17.82 & 0.12 & 32 & 0.14 & $\begin{array}{c}10 \mathrm{mM} \\
\mathrm{NaCl}\end{array}$ & $1 \mathrm{M} \mathrm{NaCl}$ & $32.72 \mathrm{~cm} / \mathrm{s}$ & $\begin{array}{c}\mathrm{A}=2.39 \mathrm{LMH} / \mathrm{bar} \\
\mathrm{B}=10.30 \times 10^{-8} \mathrm{~m} / \mathrm{s} \\
\mathrm{R}=94.1\end{array}$ & [144] \\
\hline $\begin{array}{l}\text { PAN } \\
\text { PA } \\
\text { GO }\end{array}$ & Flat sheet & 31.7 & 0.21 & 47 & 0.24 & DI water & $2 \mathrm{M} \mathrm{NaCl}$ & $300 \mathrm{ml} / \mathrm{min}$ & $\begin{array}{c}\mathrm{A}=2.04 \mathrm{LMH} / \mathrm{bar} \\
\mathrm{B}=0.83 \mathrm{LMH} \\
\mathrm{S}=0.085 \mathrm{~mm} \\
\mathrm{R}=86.43\end{array}$ & [163] \\
\hline $\begin{array}{c}\mathrm{PSF} \\
\mathrm{PA} \\
\mathrm{TiO}_{2} \\
\end{array}$ & Flat sheet & 26 & 0.19 & 34.4 & 0.18 & $\begin{array}{c}10 \mathrm{mM} \\
\mathrm{NaCl}\end{array}$ & $\begin{array}{r}0.5 \mathrm{M} \\
\mathrm{NaCl}\end{array}$ & $300 \mathrm{~mL} / \mathrm{min}$ & & [164] \\
\hline $\begin{array}{c}\text { PSF } \\
\text { PA } \\
\text { MOF ( UiO-66) }\end{array}$ & Flat sheet & 27 & 0.23 & 51.3 & 0.24 & DI water & $2 \mathrm{M} \mathrm{NaCl}$ & $1.1 \mathrm{~cm} / \mathrm{s}$ & $\begin{array}{c}\mathrm{A}=3.3 \mathrm{LMH} / \mathrm{bar} \\
\mathrm{B}=0.3 \mathrm{LMH} \\
\mathrm{S}=1637 \mu \mathrm{m} \\
\mathrm{R}=95.3\end{array}$ & [165] \\
\hline $\begin{array}{c}\text { PES } \\
\text { PA } \\
\text { MOF ( UiO-66) }\end{array}$ & Flat sheet & 47 & 0.14 & & & DI water & $2 \mathrm{M} \mathrm{NaCl}$ & $21 \mathrm{~cm} / \mathrm{s}$ & $\begin{array}{c}\mathrm{A}=4.8 \mathrm{LMH} / \mathrm{bar} \\
\mathrm{B}=0.6 \mathrm{LMH}\end{array}$ & [150] \\
\hline $\begin{array}{c}\text { PSF } \\
\text { PA } \\
\text { GO }\end{array}$ & Flat sheet & 14.5 & 0.17 & 34.7 & 0.13 & DI water & $1 \mathrm{M} \mathrm{NaCl}$ & $25000 \mathrm{ml} / \mathrm{min}$ & $\begin{array}{c}\mathrm{A}=6.52 \times 10^{-12} \mathrm{~m} / \mathrm{s} \mathrm{pa} \\
\mathrm{B}=18.7 \times 10^{-8} \mathrm{~m} / \mathrm{s} \\
\mathrm{R}=88\end{array}$ & [166] \\
\hline
\end{tabular}


Table 6(continued)

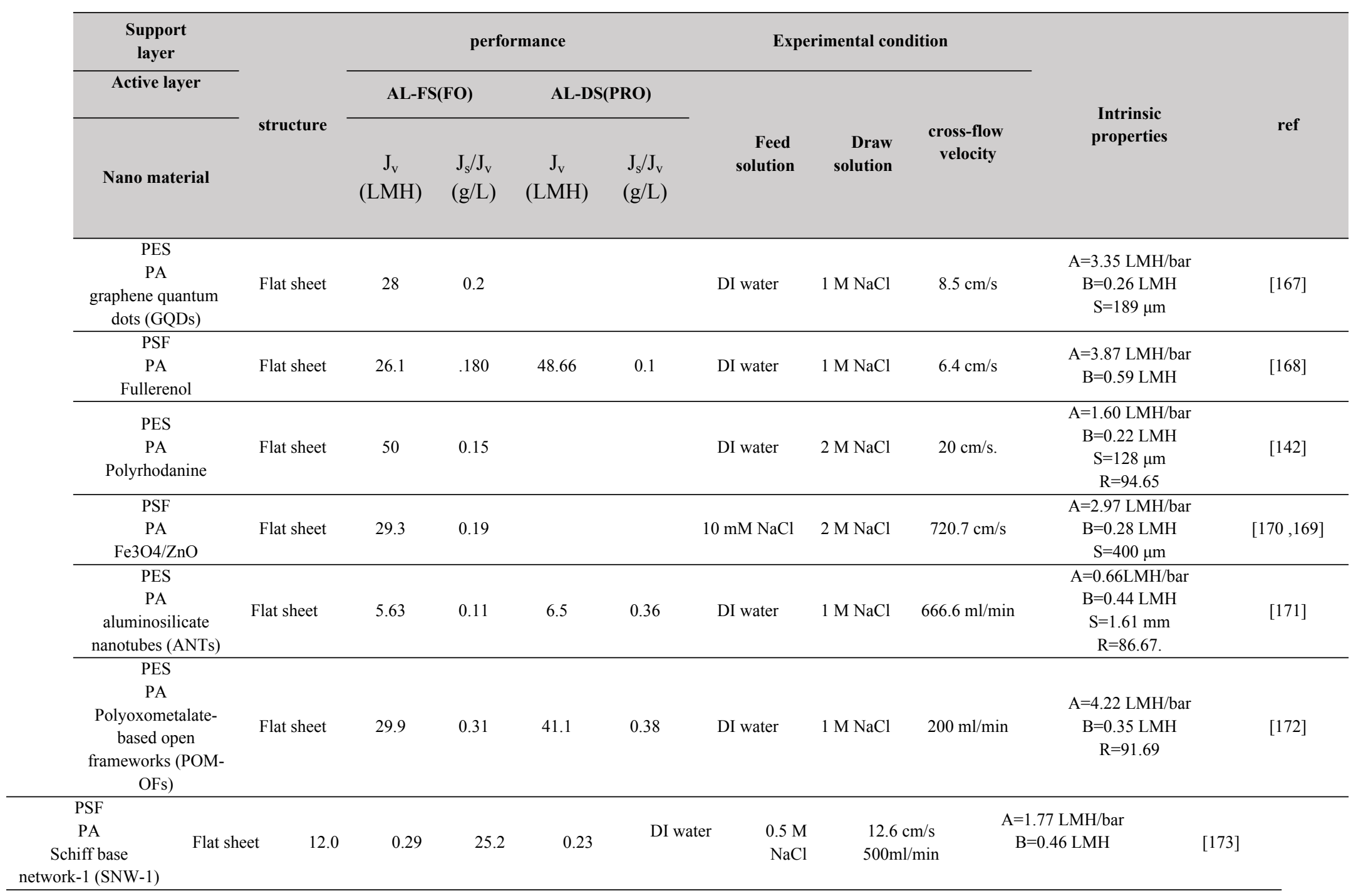


3 Table 6(continued)

\begin{tabular}{|c|c|c|c|c|c|c|c|c|c|c|}
\hline Support & \multirow[b]{3}{*}{ structure } & \multicolumn{4}{|c|}{ performance } & \multicolumn{3}{|c|}{ Experimental condition } & \multirow[b]{3}{*}{$\begin{array}{c}\text { Intrinsic } \\
\text { properties }\end{array}$} & \multirow[b]{3}{*}{ ref } \\
\hline Active layer & & \multicolumn{2}{|c|}{ AL-FS(FO) } & \multicolumn{2}{|c|}{ AL-DS(PRO) } & \multirow[b]{2}{*}{$\begin{array}{l}\text { Feed } \\
\text { solution }\end{array}$} & \multirow[b]{2}{*}{$\begin{array}{r}\text { Draw } \\
\text { solution }\end{array}$} & \multirow[b]{2}{*}{$\begin{array}{c}\text { cross-flow } \\
\text { velocity }\end{array}$} & & \\
\hline Nano material & & $\begin{array}{r}\mathrm{J}_{\mathrm{v}} \\
(\mathrm{LMH})\end{array}$ & $\begin{array}{r}\mathrm{J}_{\mathrm{S}} / \mathrm{J}_{\mathrm{V}} \\
(\mathrm{g} / \mathrm{L})\end{array}$ & $\begin{array}{r}\mathrm{J}_{\mathrm{v}} \\
(\mathrm{LMH})\end{array}$ & $\begin{array}{l}\mathrm{J}_{\mathrm{s}} / \mathrm{J}_{\mathrm{v}} \\
(\mathrm{g} / \mathrm{L})\end{array}$ & & & & & \\
\hline $\begin{array}{c}\text { PES } \\
\text { PA } \\
\text { MOF } \\
\text { (copper 1,4- } \\
\text { benzenedicarboxylate } \\
\text { nanosheets, CuBDC- } \\
\text { NS) }\end{array}$ & Flat sheet & 27.4 & 0.11 & 49.3 & 0.11 & DI water & $\begin{array}{l}1 \mathrm{M} \\
\mathrm{NaCl}\end{array}$ & $15 \mathrm{~cm} / \mathrm{s}$ & $\begin{array}{c}\mathrm{A}=3.13 \mathrm{LMH} / \mathrm{bar} \\
\mathrm{B}=0.317 \mathrm{LMH} \\
\mathrm{S}=366\end{array}$ & {$[174]$} \\
\hline $\begin{array}{c}\text { PES } \\
\text { PA } \\
\text { polyoxometalate } \\
\text { based open } \\
\text { frameworks (POM- } \\
\text { OFs) } \\
\end{array}$ & Flat sheet & 26.7 & 0.32 & 46.4 & 0.32 & DI water & $\begin{array}{l}1 \mathrm{M} \\
\mathrm{NaCl}\end{array}$ & $200 \mathrm{ml} / \mathrm{min}$ & & {$[175]$} \\
\hline $\begin{array}{c}\text { PES } \\
\text { PA } \\
\text { mesoporous silica } \\
\text { modified with amine } \\
\text { (SBA-15-NH2) }\end{array}$ & Flat sheet & 55.3 & 0.3 & 80.4 & 0.28 & DI water & $\begin{array}{l}1 \mathrm{M} \\
\mathrm{NaCl}\end{array}$ & $200 \mathrm{ml} / \mathrm{min}$ & & {$[176]$} \\
\hline $\begin{array}{c}\text { PES } \\
\text { PA } \\
\text { GO-oxidized carbon } \\
\text { nanotubes (OCNTs) } \\
\end{array}$ & Flat sheet & 84.6 & 0.04 & 114 & 0.04 & DI water & $\begin{array}{l}1 \mathrm{M} \\
\mathrm{NaCl}\end{array}$ & $\begin{array}{l}1500 \\
\mathrm{ml} / \mathrm{min}\end{array}$ & & {$[177]$} \\
\hline $\begin{array}{c}\text { PES } \\
\text { PA } \\
\text { GQDs@UiO-66- } \\
\text { NH2 }\end{array}$ & Flat sheet & 59.3 & 0.32 & 85.3 & 0.42 & DI water & $\begin{array}{l}1 \mathrm{M} \\
\mathrm{NaCl}\end{array}$ & $200 \mathrm{ml} / \mathrm{min}$ & $\begin{array}{c}\mathrm{A}=4.88 \mathrm{LMH} / \mathrm{bar} \\
\mathrm{B}=1.356 \mathrm{LMH} \\
\mathrm{S}=366\end{array}$ & {$[178]$} \\
\hline
\end{tabular}


2 Table 7: A summary of the studies on TFC-N with modified both substrate and active layer

\begin{tabular}{|c|c|c|c|c|c|c|c|c|c|c|}
\hline \multirow{2}{*}{$\begin{array}{c}\begin{array}{c}\text { Support } \\
\text { layer }\end{array} \\
\text { Active layer }\end{array}$} & \multirow[b]{3}{*}{ structure } & \multicolumn{4}{|c|}{ performance } & \multicolumn{3}{|c|}{ Experimental condition } & \multirow[b]{3}{*}{$\begin{array}{c}\text { Intrinsic } \\
\text { properties }\end{array}$} & \multirow[b]{3}{*}{ ref } \\
\hline & & \multicolumn{2}{|c|}{ AL-FS(FO) } & \multicolumn{2}{|c|}{ AL-DS(PRO) } & \multirow[b]{2}{*}{$\begin{array}{c}\text { Feed } \\
\text { solution }\end{array}$} & \multirow[b]{2}{*}{$\begin{array}{c}\text { Draw } \\
\text { solution }\end{array}$} & \multirow[b]{2}{*}{$\begin{array}{c}\text { cross-flow } \\
\text { velocity }\end{array}$} & & \\
\hline Nano material & & $\begin{array}{c}\mathrm{J}_{\mathrm{v}} \\
(\mathrm{LMH})\end{array}$ & $\begin{array}{c}\mathrm{J}_{\mathrm{s}} / \mathrm{J}_{\mathrm{v}} \\
(\mathrm{g} / \mathrm{L})\end{array}$ & $\begin{array}{c}\mathrm{J}_{\mathrm{v}} \\
(\mathrm{LMH})\end{array}$ & $\begin{array}{l}\mathrm{J}_{\mathrm{s}} / \mathrm{J}_{\mathrm{V}} \\
(\mathrm{g} / \mathrm{L})\end{array}$ & & & & & \\
\hline PSF & & & & & & & & & $\mathrm{A}=8.43 \mathrm{LMH} / \mathrm{bar}$ & \\
\hline $\begin{array}{c}\text { PA } \\
\text { aluminum oxide }\left(\mathrm{Al}_{2} \mathrm{O}_{3}\right)\end{array}$ & Flat sheet & 27.6 & 0.25 & 51.5 & 0.05 & DI water & $1 \mathrm{M} \mathrm{NaCl}$ & $18.5 \mathrm{~cm} / \mathrm{s}$ & $\begin{array}{c}\mathrm{B}=1.66 \mathrm{LMH} \\
\mathrm{S}=1028 \mu \mathrm{m}\end{array}$ & [179] \\
\hline $\begin{array}{c}\text { PSF } \\
\text { PA } \\
\text { HNTs } \\
\text { g-C3N4 }\end{array}$ & Flat sheet & 18.88 & 0.14 & & & DI water & $2 \mathrm{M} \mathrm{NaCl}$ & $21.4 \mathrm{~cm} / \mathrm{s}$ & $\begin{array}{c}\mathrm{A}=2.171 \mathrm{LMH} / \mathrm{bar} \\
\mathrm{B}=10.5 \times 10^{-8} \mathrm{~m} / \mathrm{s} \\
\mathrm{S}=0.37 \mathrm{~mm} \\
\mathrm{R}=93\end{array}$ & [149] \\
\hline $\begin{array}{c}\text { CA } \\
\text { PA } \\
\text { Hapf }\end{array}$ & Flat sheet & & & 22.6 & 0.6 & DI water & $1 \mathrm{M} \mathrm{NaCl}$ & & $\begin{array}{c}\mathrm{A}=1.41 \mathrm{LMH} / \mathrm{bar} \\
\mathrm{B}=1.62 \mathrm{LMH} \\
\mathrm{S}=0.865 \mathrm{~mm} \\
\mathrm{R}=92.23\end{array}$ & [152] \\
\hline
\end{tabular}




\subsection{Performance of TFC-D membranes}

One of the most important developments in the production of FO membranes is producing doubleskinned membranes to solve the problems caused by fouling and concentration polarization without changing the AL-DS orientation (because in this direction a higher flux can be achieved) [180]. Both laboratory studies and modeling have proven the effectiveness of TFC-D's [181-184]. In FO operation, one of the skin layers is in contact with the draw solution, acting as the semipermeable membrane, while the other layer is in contact with the feed solution, preventing the foulants from entering the porous substrate. [181]. Wang et al. [182] were the first to develop a double-skinned membrane from CTA for FO. Fig. 23 shows the schematic image of a doubleskinned FO membrane. $A_{\text {draw }}$ is the water permeability of the active layer of the draw solution side (draw active layer), $B_{\text {draw }}$ is the salt permeability of the active layer of the draw side, $A_{\text {feed }}$ is feed layer water permeability, $B_{\text {feed }}$ is salt permeability of feed layer, $\pi_{s / d}$ and $C_{s / d}$ are the osmotic pressure and solute concentration at the interface between draw active layer and the substrate, respectively, $\pi_{s / f}$ and $C_{s / f}$ are the osmotic pressure and the solute concentration at the interface between the substrate and the feed active layer, respectively, and finally $P_{\text {support }}$ is the hydraulic pressure in the substrate layer [181].

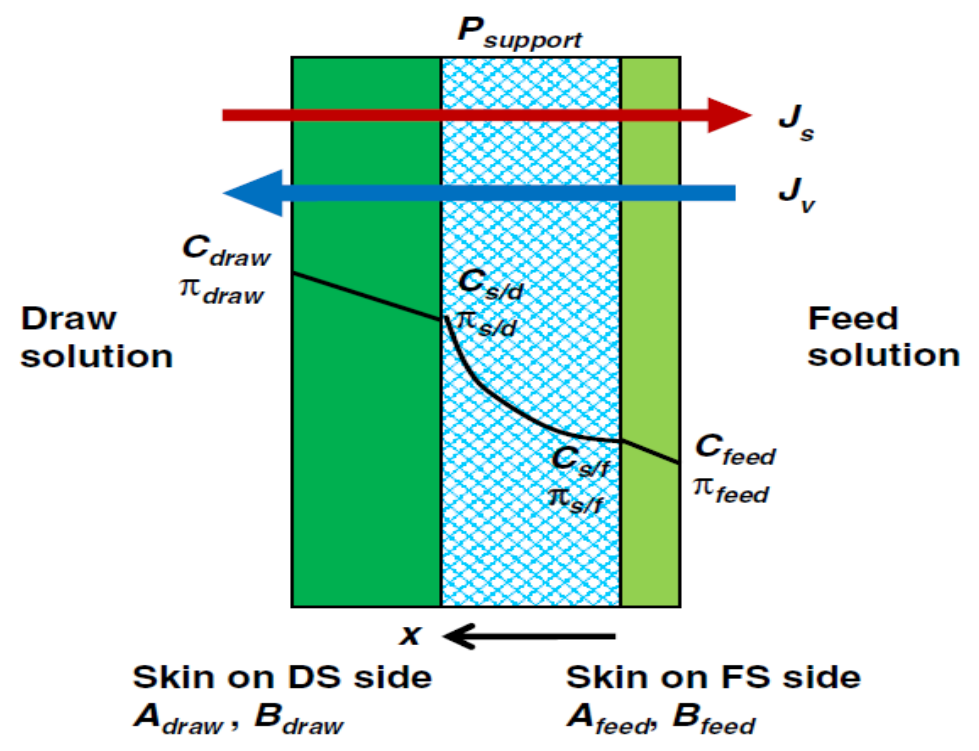

Fig 23: schematic of Double-skinned membrane [181]

\subsubsection{Inner active layer RO polyamide and outer skin layer NF polyethyleneimine}

Fang et al. [180] developed a new TFC membrane in the form of a TFC-D. An active layer similar to the typical active layer used in RO and another active layer similar to a NF active layers were formed onto the two sides of poly (amide-imide) (PAI) hollow fiber substrate. The inner active layer (RO) was made from polyamide by interfacial polymerization, and the outer respectively. Additionally, two PAI hollow fiber substrates, PAI\#1 and PAI\#2 were spun by 
changing the bore fluid flow rate and air gap using constant spinning dope composition, as shown in Table 8. Using these substrates, three TFC-D hollow fibers were fabricated, i.e. $\mathrm{PAI} \# 1-\mathrm{NF} / \mathrm{RO}, \mathrm{PAI} \# 1-\mathrm{RO} / \mathrm{NF}$ and PAI\#2-RO/NF. The membrane codes indicate the substrate used and the sequence of skin layer formation, e.g. NF/RO means the outer active layer (NF) was formed first, followed by the inner layer (RO) formation.

Table 8: Spinning conditions and parameters [180]

\begin{tabular}{lcc}
\hline \multicolumn{1}{c}{ Parameters } & PAI\#1 & PAI\#2 \\
\hline Dope composition (PAI/LiCl/NMP) (wt.\%) & $14 / 4 / 82$ & $14 / 4 / 82$ \\
\hline Dope flow rate $(\mathrm{g}$ min-1) & 6.0 & 6.0 \\
\hline Bore fluid (NMP/H2O) $(\mathrm{vol} . \%)$ & $25 / 75$ & $25 / 75$ \\
\hline Bore fluid flow rate $(\mathrm{mL}$ min-1) & 7.0 & 6.0 \\
\hline Air gap $(\mathrm{cm})$ & 5.0 & 2.0 \\
\hline Take up speed & Free fall & Free fall \\
\hline External coagulant & Tap water & Tap water \\
\hline Spinning temperature $\left({ }^{\circ} \mathrm{C}\right)$ & 23 & 23 \\
\hline Spinneret diameter $(\mathrm{mm})$ & 1.5 & 1.5 \\
\hline ID of bore fluid needle $(\mathrm{mm})$ & 0.7 & 0.7 \\
\hline
\end{tabular}

The morphology of the substrates of the membranes was studied by SEM. Fig. 24 shows SEM 11
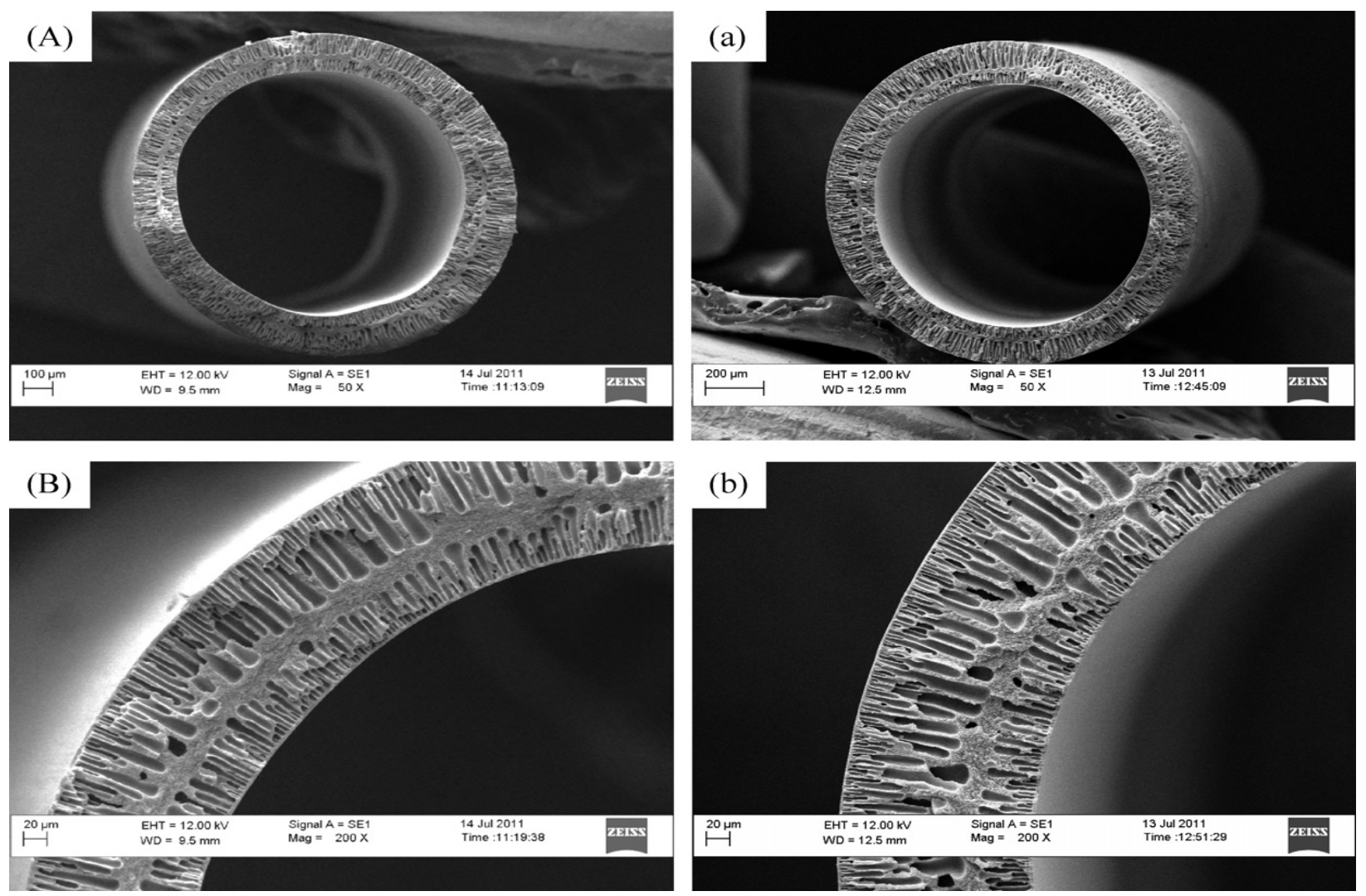

Fig 24: Cross-section morphologies of PAI hollow fiber substrates: (A) PAI\#1 at 50×; (B) PAI\#1 enlarged at 200×;

(a) PAI\#2 at 50×; (b) PAI\#2 enlarged at 200× [180] 
1 Fig. 24 illustrates that the cross-section of the substrates is divided into three regions, including a 2 narrow area of the sponge-like structure at the middle which was sandwiched between two wider 3 finger-like sections. The middle layer of the substrate PAI\#2 is thinner than that of PAI\#1 substrate 4 due to its shorter air gap. The shorter air gap, caused the spun solution to come into contact with 5 the external coagulant (water) sooner [180]. Indeed, before entering the spun fiber into the external 6 coagulation bath, the presence of water vapor in the ambient air induced a slight phase inversion 7 at the outer surface. This led to increase the viscosity at the outer surface, hence, a much thicker 8 skin was obtained [185]. Because the bore fluid non-solvent was weaker than the external 9 coagulant fluid, the finger-like structure developed from the outer surface was deeper than that of 10 the inner surface one. Hollow fibers with the larger fraction of finger-like layer is more desirable 11 due to the higher porosity hence higher membrane productivity.

12 The performance of the three TFC-D hollow fiber membranes was studied. They used $\mathrm{NaCl}$ draw 13 solution of 0.5 to 2 molar concentrations and deionized water as the feed in both AL-DS and AL14 FS orientation, as the results are shown in Fig. 25. From the figure, PAI\#2-RO/NF exhibits the highest water flux in both orientations likely due to the higher porosity of PAI\#2 substrate. 16 Comparing AL-DS and AL-FS orientation, AL-DS demonstrated higher water flux, as expected. $17 \mathrm{PAI}+2-\mathrm{RO} / \mathrm{NF}$ in AL-DS orientation also showed the lowest solute flux/water flux $\left(J_{s} / J_{v}\right)$ ratio. 18 Finally, by comparing the performance of the fabricated membranes in this work with those of other studies, Fang et al. concluded that membranes made with two active layers have better performance than the membranes with only one active layer when the feed solution containing 21 bivalent cations causes membrane fouling. In addition, it was concluded that the TFC-D hollow fiber membrane demonstrated better performance than the TFC-D flat sheet membranes. It is 23 presumably due to higher intrinsic properties of hollow fiber membrane in comparison to flat sheet 24 membrane as a substrate [180]. 
(a) Water flux for AL-DS orientation

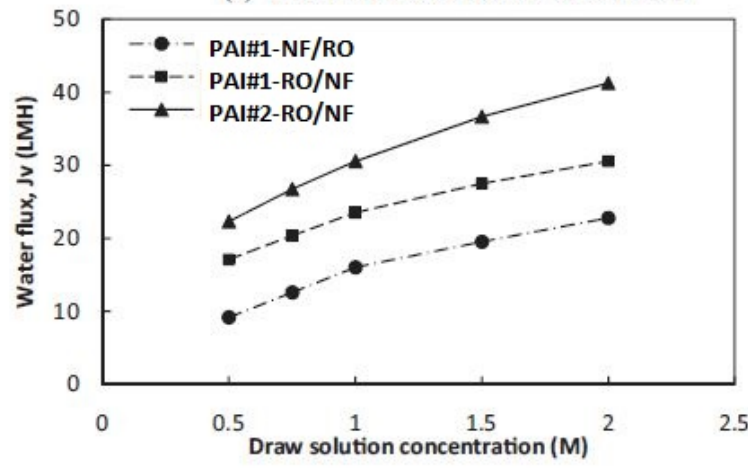

(b) Water flux for AL-FS orientation

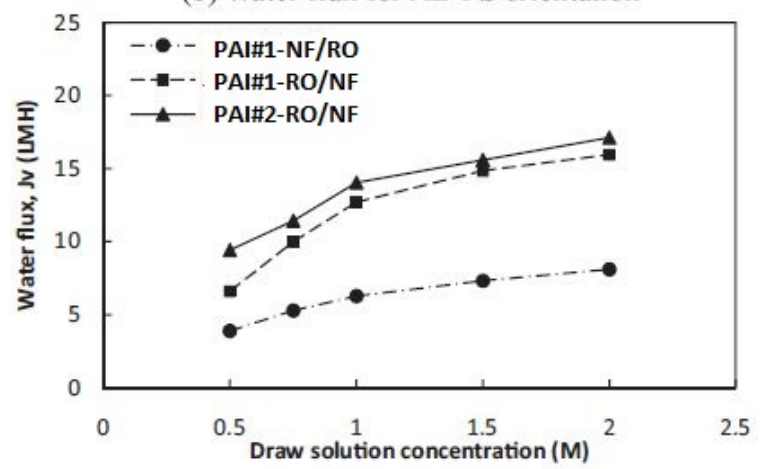

(c) $\mathrm{J}_{\mathrm{s}} / \mathrm{J}_{\mathrm{v}}$ for AL-DS orientation

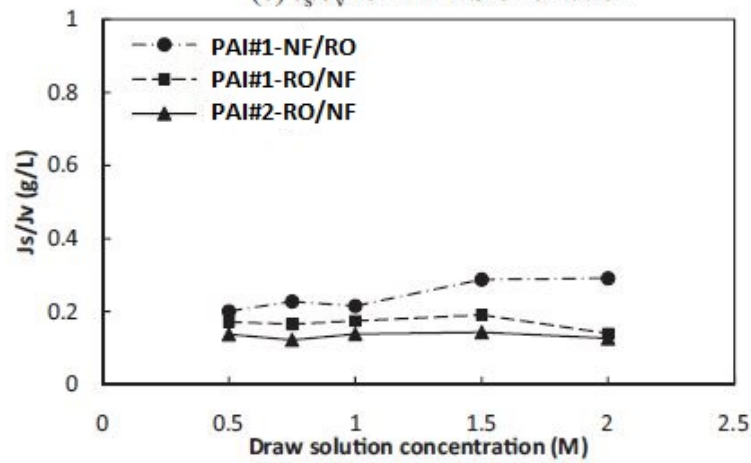

(d) $J_{s} / J_{v}$ for AL-FS orientation

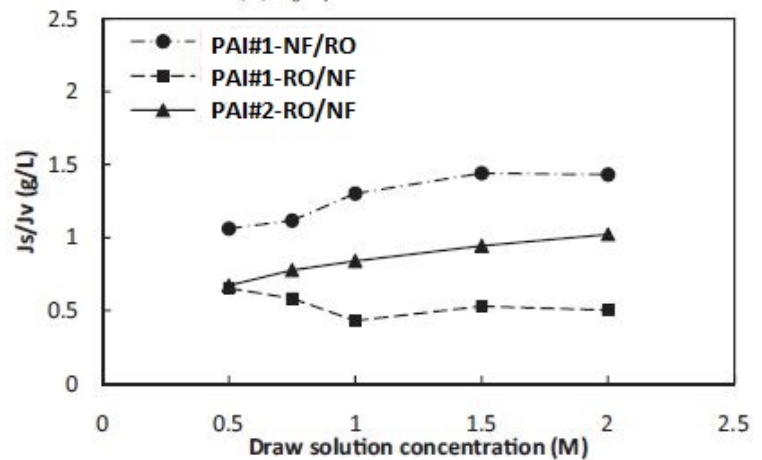

Fig 25: FO performance of TFC-D hollow fiber membranes: Draw solution: 0.5-2.0 M NaCl; feed: DI water. (a) Water flux for AL-DS (PRO) orientation, (b) water flux for AL-FS(FO) orientation, (c) Js/Jv for AL-DS orientation and (d) Js/Jv for AL-FS orientation [180]

Fig. 25 also depicts the effect of the sequence of the inner and outer layer formation on FO performance, i.e. the water flux of PA\#1-NF/RO is lower than PA\#1-RO/NF. When NF membrane is first formed on the outer layer with chemical modification by PEI, a large number of free amine groups from PEI was attached onto the cross-linked outer skin. During the formation of the second skin (RO) on the inner surface by interfacial polymerization of TMC and MPD, most likely the excess of TMC monomers permeated through the substrate and contacted with the free amines of PEI left at the outer layer, resulting in denser outer layer. On the other hand, when interfacial polymerization was occurred first, the excess TMC monomers were quickly altered to carboxylic acid and their reactivity was reduced before subsequent PEI skin layer was fabricated. Consequently, the fabrication of the two dense skin layers has less interference with each other [180]. It is also possible that no monomers remain unreacted after the interfacial polymerization.

\subsubsection{Both skin layers from polyamide}

Han et al. [184] developed a hollow fiber membrane with two polyamide active layers formed on the two sides of PES substrate to control the internal fouling and concentration polarization. The active layer on the inner surface acted as the selective layer, while the outer active layer just prevented foulants from entering into the substrate. The average pore size of the second active 
layer is $0.7 \mathrm{~nm}$ with narrow pore size distribution, which is sufficient to prevent the foulants from entering the substrate pore. Two feeds, wastewater and deionized water, were used together with $1 \mathrm{M} \mathrm{NaCl}$ as draw solution. When the membrane with one selective layer is applied in AL-DS (PRO) orientation, the substrate is in contact with the feed and thus foulants can easily enter the porous substrate (see Fig.26 (a)), resulting in severe internal concentration polarization and fouling, which reduces the flux. As a result, removing the foulants from the membrane becomes more challenging. The TFC-D could prevent the foulant intrusion into the substrate, as shown in 8 Fig. 26 (b). Although the external concentration polarization (ECP) is intensified in this case because of the second active layer, the negative effect of ECP is less severe than ICP in FO. Moreover, the foulants deposited on top of the polyamide layer could be easily washed away [184].

(a)

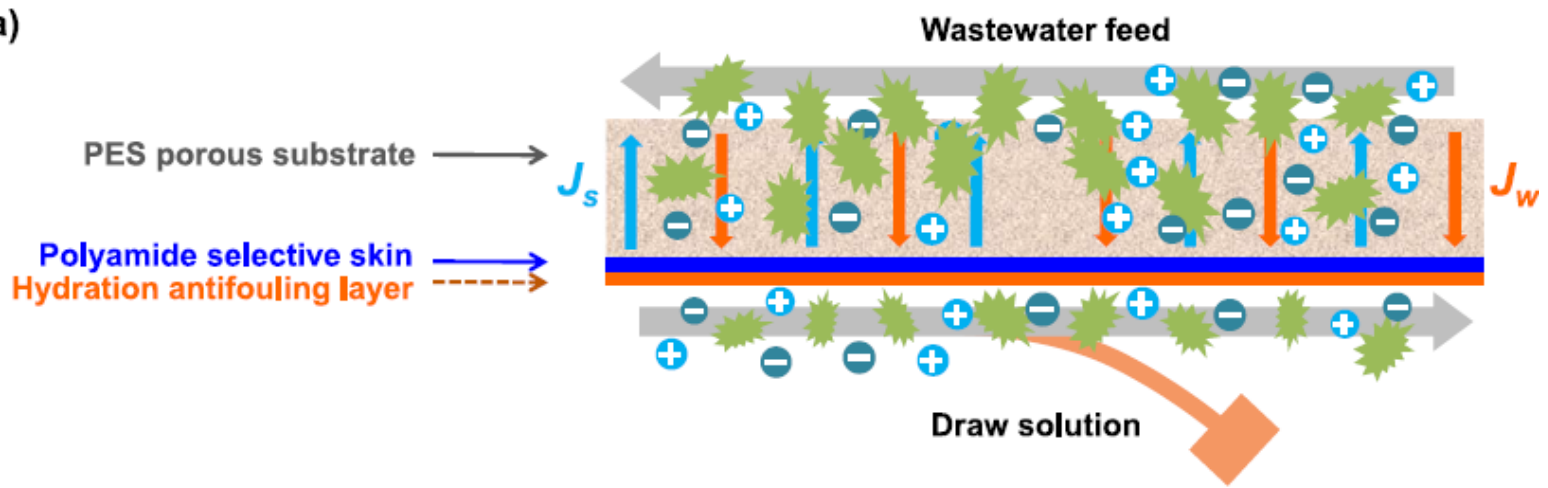

(b)

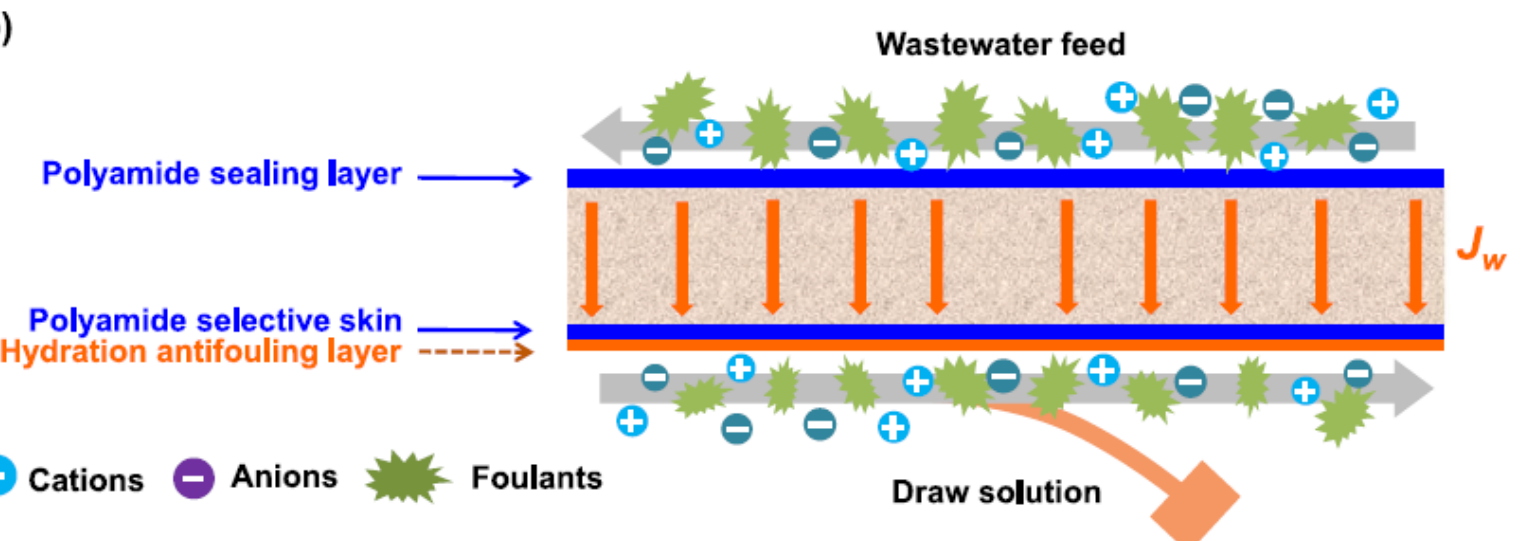

Fig 26: Schematic of fouling phenomena: (a) the conventional TFC membrane and (b) the newly developed TFC-D membrane in FO under the PRO mode [184].

Table 9 shows the review of literature on the development of TFC-D membranes.

\subsection{Issues and implications of TFC-D membranes}

Although, TFC-D membranes show excellent properties especially regarding reverse salt flux and fouling propensity, these membranes show low water flux compared with the normal TFC membranes i.e. with single skin. Methods such as IP, layer-by-layer polyelectrolyte deposition and PDA deposition are applied to produce double-skinned FO membranes. For achieving high water 
1 flux of TFC-D membranes, it is suggested to use an RO-like active layer on one side and an NF

2 active layer on the other side. Fabricating two RO-like active layer on the two side can drastically

3 decrease the water flux. It is also recommended to apply nanomaterial in one or both of the active 4 layers to compensate the decline in water flux. 
2 Table 9: Summary of Double-skinned TFC FO membranes a

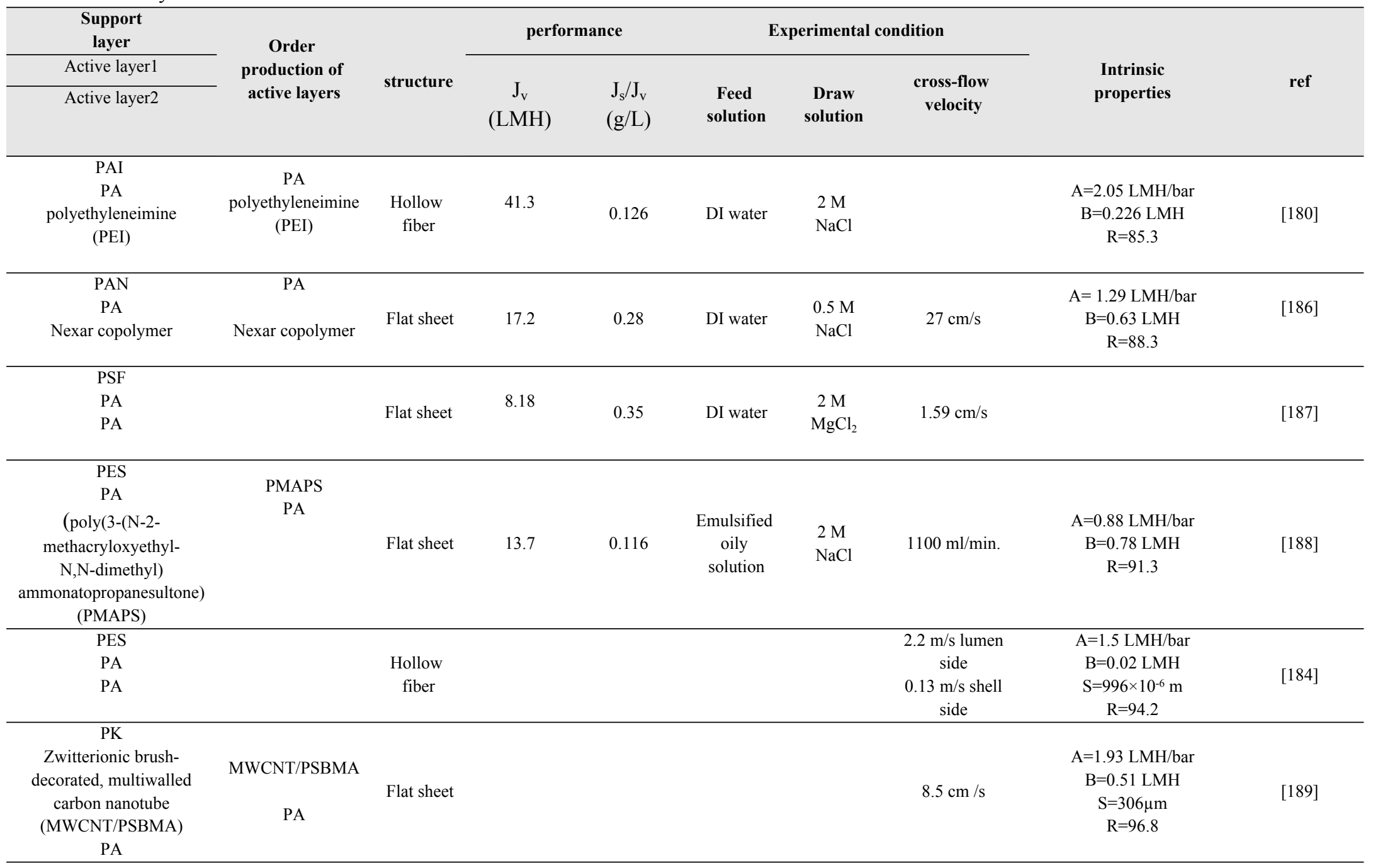

a: data is reported in AL-DS orientation 


\section{Commercial FO membranes}

Despite many membranes that have been designed by various scientific groups specifically for FO process, it seems FO technology still needs membranes of higher performance. Fortunately, however, some of the membranes have already been commercialized. The commercial membranes are mostly in flat sheet form [37]. Table 10 summarizes the current commercial FO membranes. Although, some papers [190] have mentioned several other companies as FO membrane supplier, we could not successfully find reliable information from their websites.

8

Table 10: Status of the current commercial FO membranes

\begin{tabular}{lcccc}
\hline Supplier/Manufacturer & Status & $\begin{array}{c}\text { System } \\
\text { supply }\end{array}$ & $\begin{array}{c}\text { Membrane and } \\
\text { Configuration }\end{array}$ & Primary application \\
\hline HTI & Commercial & Yes & SWo (CA, TFC) & Various \\
\hline Aquaporin A/S & Commercial & No & SWo, HF (Aquaporin) & $\begin{array}{c}\text { FO, osmotic } \\
\text { concentration }\end{array}$ \\
\hline Modern Water & Commercial & Yes & SWo & Seawater FO \\
\hline Oasys Water & Commercial & Yes & SWo (TFC) & Brine concentration, ZLD \\
\hline Porifera & Commercial & Yes & SWo (TFC) & Various \\
\hline Trevi Systems & Commercial & Yes & SWo & Seawater FO \\
\hline Green Centre Canada & Development & No & SWo & Seawater FO \\
\hline Idaho National Lab & Development & No & NA & Reverse osmosis \\
\hline Fluid Technology solutions & Commercial & NA & SWo (CTA) & Wastewater treatment \\
\hline IDE Technologies & Precommercial & Yes & SWo & Press.-retarded osmosis \\
\hline $\begin{array}{l}\text { NA: not available; SWo :spiral wound, HF: hollow fiber, ZLD: Zero liquid discharge } \\
\text { a Demonstration-scale FO membrane treatment system available (yes/no) }\end{array}$ & & & & \\
\hline
\end{tabular}

In contrast to the membranes listed in Table 1 HTI Co. commercialized the FO membranes for the first time. Unfortunately, they no longer supply their products [46]. However, because of their historic importance, the details of their membranes are given below.

\subsection{HTI membrane}

Ren et al. [191] characterized and tested the performance of a TFC membrane developed by HTI Co. The FESEM images of the active layer and the substrate surface at different magnifications are shown in Fig. 27. The active layer has a ridge and valley morphology which is a typical feature of polyamide active layers formed by interfacial polymerization. The active layer seems uniform and defect-free. The membrane substrate is porous with pore sizes from 100 to $600 \mathrm{~nm}$. Moreover, they soaked the TFC membrane in 50\% wt. isopropyl alcohol (IPA) solution at room temperature for 5 minutes. Then, isopropyl alcohol was completely washed out with deionized water in which the membrane was stored at a temperature of $5{ }^{\circ} \mathrm{C}$. The performance of this soaked membrane was compared with that of the non-soaked TFC membrane. The water and salt permeability increased in by soaking. When the membrane was soaked in IPA, no chemical reaction occurred between polyamide and IPA, but the molecules of low molecular weight were removed from the active 
layer, thereby forming a more open-structured polyamide active layer. Also, in the presence of 2 IPA, the swelling of polyamide chains has increased due to the hydrogen bonding between IPA 3 and polyamide and the affinity of IPA to the nonpolar part of polyamide. Therefore, the interaction 4 between the polymer chains was weakened in the active layer and the chains became more 5 flexible, which in turn increased the pore size. The presence of larger pores and open structure 6 increase simultaneously the permeability of water and salt.
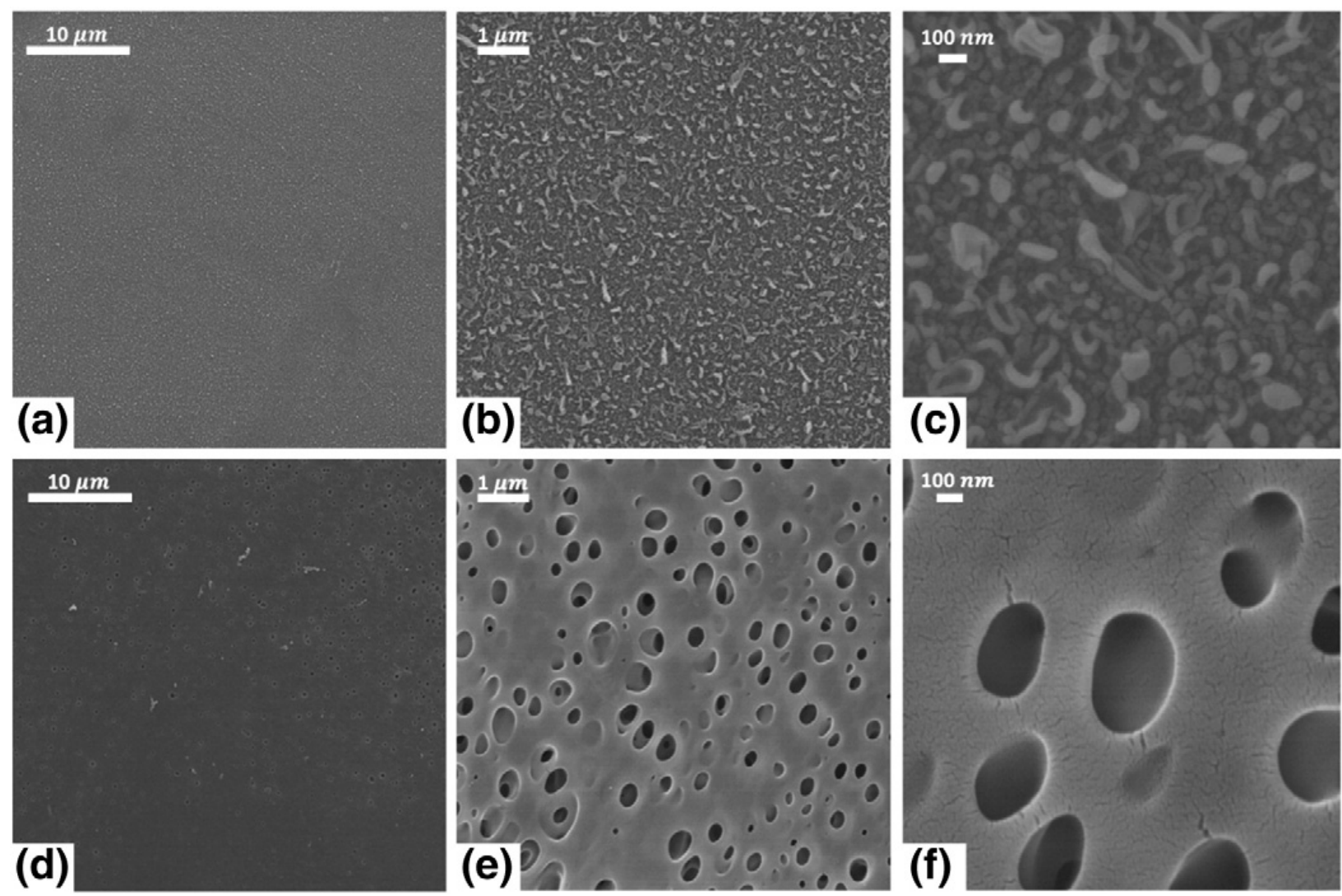

Fig 27: Top surface SEM images ( $a, b$ and $c$ ) and bottom surface FESEM images (d, e and $f$ ) of TFC membrane developed by HTI Co. at magnifications of (a and d) 2000×, (b and e) 10,000×, and (c and f) 50,000× [191]

\subsection{Aquaporin A/S membrane}

Ren el al. [192] reported their research on the performance of the hollow fiber FO membrane supplied by Aquaporin A/S. The small-scale modules were applied for testing the membrane under various testing conditions. They incorporated aquaporin into the selective layer of the hollow fibers at the lumen surface. These membranes presented excellent FO performance. Fig. 28 illustrates the cross-sectional morphology of the membranes at different magnifications. The entire structure of the hollow fiber membranes was sponge-like structure. Sponge-like structure would be useful for long term operation because of its higher mechanical stability. 

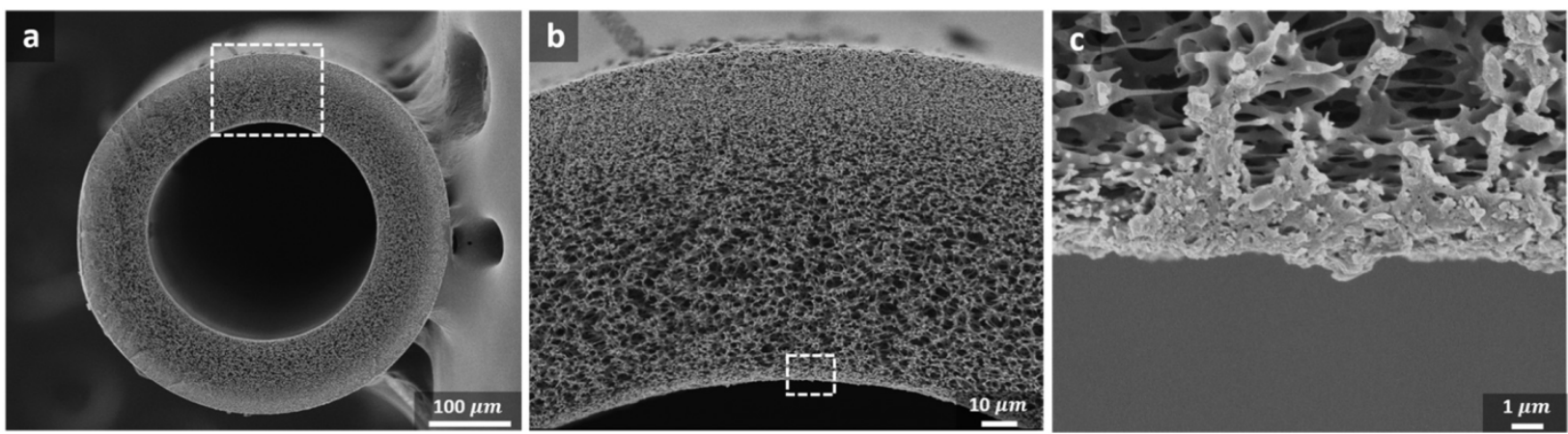

Fig. 28. Cross-section FESEM images of a hollow fiber membrane at (a) $170 \times$; (b) $800 \times$; (c) $5000 \times$.

Table 11 shows the results on the water flux and the specific reverse salt flux of the current commercial FO membranes.

Table 11: Summary of the studies on the current commercial FO membranes

\begin{tabular}{|c|c|c|c|c|c|c|c|c|}
\hline \multirow{3}{*}{$\begin{array}{l}\text { Membrane } \\
\text { supplier }\end{array}$} & \multirow[b]{3}{*}{ structure } & \multicolumn{4}{|c|}{ performance } & \multicolumn{2}{|c|}{ Experimental condition } & \\
\hline & & \multicolumn{2}{|c|}{ AL-FS(FO) } & \multicolumn{2}{|c|}{ AL-DS(PRO) } & \multirow[b]{2}{*}{$\begin{array}{c}\text { Feed } \\
\text { solution }\end{array}$} & \multirow[b]{2}{*}{$\begin{array}{r}\text { Draw } \\
\text { solution }\end{array}$} & \\
\hline & & $\begin{array}{c}\mathrm{J}_{\mathrm{v}} \\
(\mathrm{LMH})\end{array}$ & $\begin{array}{l}\mathrm{J}_{\mathrm{s}} / \mathrm{J}_{\mathrm{v}} \\
(\mathrm{g} / \mathrm{L})\end{array}$ & $\begin{array}{c}\mathrm{J}_{\mathrm{v}} \\
(\mathrm{LMH})\end{array}$ & $\begin{array}{c}\mathrm{J}_{\mathrm{S}} / \mathrm{J}_{\mathrm{V}} \\
(\mathrm{g} / \mathrm{L})\end{array}$ & & & \\
\hline HTI & Flat sheet & 21.10 & 0.13 & 31.61 & 0.45 & DI water & $1 \mathrm{M} \mathrm{NaCl}$ & [191] \\
\hline $\begin{array}{c}\text { Prewetted } \\
\text { HTI }\end{array}$ & Flat sheet & 23.03 & 0.27 & 46.69 & 0.52 & DI water & $1 \mathrm{M} \mathrm{NaCl}$ & [191] \\
\hline Toyobo & Hollow fiber & 8 & 0.07 & 15 & N/A & DI water & $1 \mathrm{M} \mathrm{NaCl}$ & [193] \\
\hline $\begin{array}{c}\text { Aquaporin } \\
\mathrm{A} / \mathrm{S}\end{array}$ & Hollow fiber & 13.2 & 0.14 & 21 & .18 & DI water & $1 \mathrm{M} \mathrm{NaCl}$ & {$[192]$} \\
\hline
\end{tabular}

\section{Proposing roadmap, concluding remarks and future directions}

In this paper, recent developments in TFC FO membranes, as an emerging process considering its challenges and capabilities were reviewed. A number of studies were considered on the development and design of the membranes and the challenges during its development stages were discussed. TFC FO membranes should have a robust but thin substrate and a well-organized active layer with a high-water permeability and selectivity which can help to make FO as a practical competitive process in desalination and water treatment applications.

Based upon Fig. 1, it is obvious that up to this point three major methods have been developed by researchers to fabricate TFC FO membranes. In order to have a correct understanding of the superiority of each of the membrane groups, Fig. 29 as a general performance curve and a road map for TFC-FO membranes was generated. The data for TFC-A membranes, TFC-N membranes and TFC-D membranes were taken from Tables 1 and 3, 6 and 9, respectively. Fig. 29a depicts FO reverse salt flux $/ \Delta \pi\left(J_{S} / \Delta \pi\right)$ versus FO water flux $/ \Delta \pi\left(J_{v} / \Delta \pi\right)$ almost for all the FO membranes reviewed in this work. Clearly, several important facts are shown by the figure. First, based on the available data, there is a general trend between $J_{S} / \Delta \pi$ and $J_{v} / \Delta \pi$, i.e. reverse salt flux is directly related to water flux. Disregarding the membrane preparation methods, this is true for the all three 
groups of membranes collected in this study. Second, the increasing trend is accompanied by a flat

2 slope, which is corresponding to a certain amount of the $J_{v} / \Delta \pi$ for each group. The trends of 3 substrate modified TFC-N's and TFC-A's are very similar to each other. Third, the addition of 4 nanoparticles to the active layer structure as active layer modified TFC-N's shows two different 5 features corresponding to the $J_{v} / \Delta \pi$ values before and after $\sim 0.4 \mathrm{LMH} / \mathrm{bar}$. At low $J_{v} / \Delta \pi$ the overall 6 results have been enhanced in terms of controlling the reverse salt flux compared to normal TFC 7 membranes, however, at higher $J_{v} / \Delta \pi$ the trend is reverse and the reverse salt flux increased. The 8 first part is most likely because of low nanoparticle loadings, appearing appropriate interaction 9 between the PA and the nanoparticle, whilst the second part is most probably due to the high 10 nanoparticle loadings that resulted in particle agglomeration and pinhole formation in the active 11 layer. Therefore, clearly further carefully designed studies are needed to stablish the nanoparticle 12 application in TFC-N membranes to shift the above inflection point toward higher $J_{v} / \Delta \pi$ values. 13 Interestingly, from Fig. 29, as an important progress in the trend of synthesis of FO membranes, 14 TFC-D membranes meaningfully possess the potential of controlling the reverse solute diffusion, 15 which this in turn is associated with a significant decrease in ICP. Fig. 29b illustrates the 16 meaningful gap between TFC-D membranes and TFC-A and TFC-N membranes in terms of $J_{S} / J_{v}$ 17 versus $J_{v} / \Delta \pi$. Fortunately, the trend is quite descending, whereas for the rests is ascending. 18 Therefore, this conclusion can be drawn that working on active layer modified TFC-Ns and 19 double-skinned membranes TFC-D, and investigating the correlation between the nanoparticles 20 and the membrane performance will be an important focus area in order to commercialize FO 21 membranes.

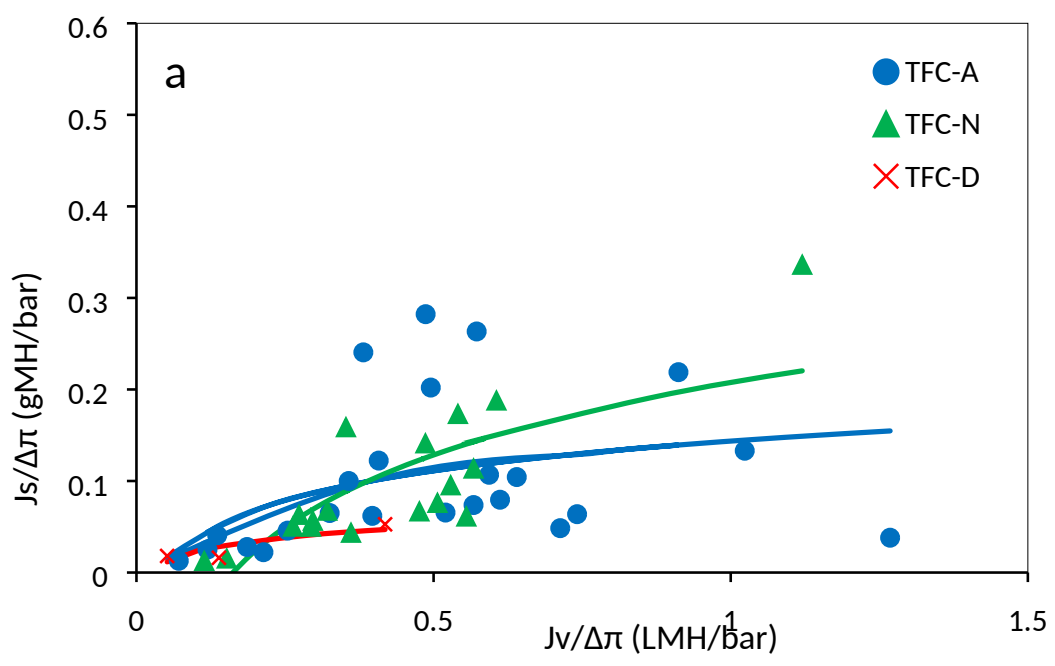




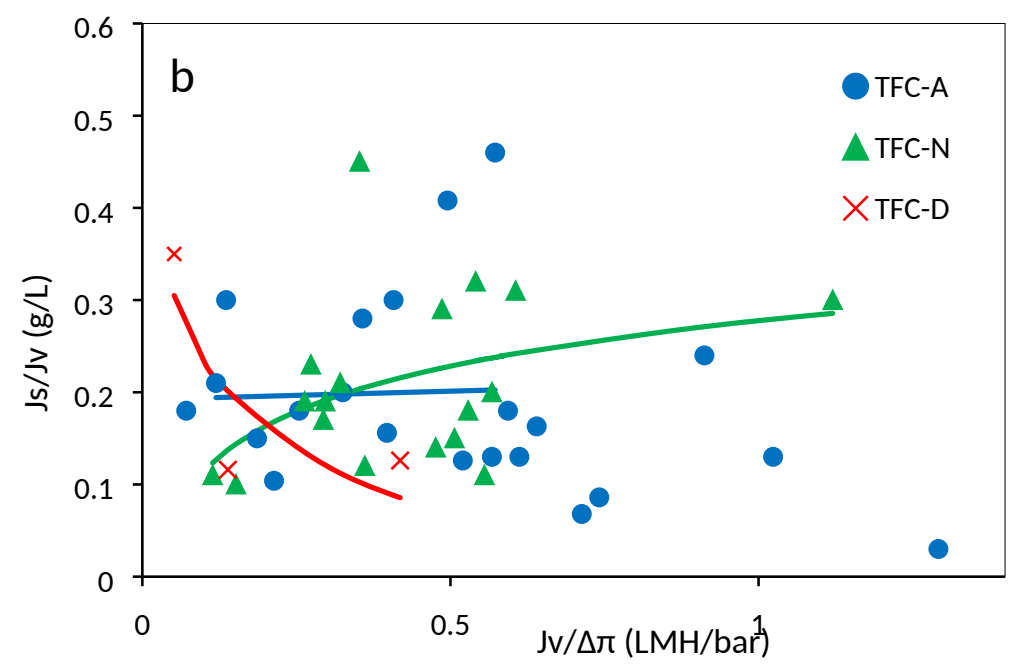

Fig. 29: Reverse salt flux versus water flux for the FO membranes reviewed in this work (TFC-A and TFC-N: ALFS mode, TFC-D: AL-DS mode) (data points are taken from Tables 1, 3,6 and 9)

According to this review, it can be concluded that the most studies on the production of TFC membranes have been conducted with deionized water as feed and a low concentration of draw solutions. For a better understanding of the membrane performance, the lab scale test conditions such as the composition of feed and draw solutions should be preferentially close to that of actual conditions. For achieving better understanding of the performance of the FO membranes Hao et al. [114] recommended that, instead of permeability-selectivity, permeability-selectivityantifouling trade-off must be considered.

In the majority of the studies, the commercial TFC FO membranes are of the flat sheet form. Considering the pronounced superiority of the hollow fiber membranes over the flat sheet membranes, it is advisable to perform more laboratory research and modeling on the application of hollow fiber membranes in FO. Also, more studies should be performed on the less considered conditions of FO process i.e. temperature of the feed and different draw solutions. The lack of study on TFC-N's is quite clear. However, the present fabricated TFC-N's exhibit high-water flux, they suffer from the shortcoming of high reverse solute diffusion. Hence, more research is necessary for controlling this undesired aftermath before commercialization of TFC-N membranes. Based on the present review, TFC-D's have a brilliant future. Therefore, more studies should be conducted on them because of their prominent potential for controlling ICP and membrane fouling. Moreover, further studies are required to reveal the role of the nanoparticles incorporating in both skins simultaneously on the performance of the membrane. Generally, selection of suitable nanomaterials is a key-parameter in TFC-N membrane formation. This selection depends on the composition of feed and draw solution. Existence of nanoscale fillers can enhance the free volume by disrupting the polymer main chains causing to higher water diffusivity enhancement [194]. From the open literature four main category of nano-particles can be observed which are suggested to be studied, namely: i) Microstructural design such as: MOF, GO, zeolitic imidazolate framework (ZIF), CNTs , single wall carbon nanotubes (SWCNTs), etc.), ii) Co- 
synthesis of advanced fillers such as: ZIF-8@GO, iii) Novel organometallic nanostructures such as: e.g. coordinated ligands, porous coordination polymers (PCPs), metal organic polyhedras (MOPs), ion-loaded macromolecules microporous, organic/inorganic hybrids, etc. and iv) Porous organic frameworks (POFs) for instance covalent organic frameworks (COFs), covalent triazine based frameworks (CTFs), porous aromatic frameworks (PAFs) and conjugated microporous polymers (CMPs) [194].

\section{Acknowledgements}

This research was funded by the Iran National Science Foundation (INSF) for the project entitled, "Development and fabrication of thin film composite and nanocomposite hollow fiber membrane for forward osmosis process" (Grant number: 98011635). The authors also gratefully acknowledge Sustainable Membrane Technology Research Group (SMTRG), Faculty of Petroleum, Gas and Petrochemical Engineering (FPGPE), Persian Gulf University (PGU), Iran for their financial support. In addition, the authors would like to thank Advanced Membrane Technology Research Centre (AMTEC), Universiti Teknologi Malayia (UTM) for their spiritual support.

\section{References}

[1] N. Akther, A. Sodiq, A. Giwa, S. Daer, H. Arafat, S. Hasan, Recent advancements in forward osmosis desalination: a review, Chemical Engineering Journal, 281 (2015) 502-522.

[2] K. Lutchmiah, A. Verliefde, K. Roest, L.C. Rietveld, E.R. Cornelissen, Forward osmosis for application in wastewater treatment: a review, Water research, 58 (2014) 179-197.

[3] D.L. Shaffer, N.Y. Yip, J. Gilron, M. Elimelech, Seawater desalination for agriculture by integrated forward and reverse osmosis: Improved product water quality for potentially less energy, Journal of membrane science, 415 (2012) 1-8.

[4] Z.-Y. Li, V. Yangali-Quintanilla, R. Valladares-Linares, Q. Li, T. Zhan, G. Amy, Flux patterns and membrane fouling propensity during desalination of seawater by forward osmosis, Water research, 46 (2012) 195-204.

[5] S. Zou, M. Qin, Z. He, Tackle reverse solute flux in forward osmosis towards sustainable water recovery: reduction and perspectives, Water research, 149 (2019) 362-374.

[6] L. Chekli, S. Phuntsho, H.K. Shon, S. Vigneswaran, J. Kandasamy, A. Chanan, A review of draw solutes in forward osmosis process and their use in modern applications, Desalination and Water Treatment, 43 (2012) 167-184.

[7] F. Lotfi, B. Samali, D. Hagare, Cleaning efficiency of the fouled forward osmosis membranes under different experimental conditions, Journal of Environmental Chemical Engineering, 6 (2018) 4555-4563.

[8] R. Alnaizy, A. Aidan, M. Qasim, Copper sulfate as draw solute in forward osmosis desalination, Journal of Environmental Chemical Engineering, 1 (2013) 424-430.

[9] J. Mulder, Basic principles of membrane technology, Springer Science \& Business Media, 2012.

[10] A. Sotto, A. Rashed, R.-X. Zhang, A. Martínez, L. Braken, P. Luis, B. Van der Bruggen, Improved membrane structures for seawater desalination by studying the influence of sublayers, Desalination, 287 (2012) 317-325. 
[11] F.M. Munshi, J. Church, R. McLean, N. Maier, A.A. Sadmani, S.J. Duranceau, W.H. Lee, Dewatering algae using an aquaporin-based polyethersulfone forward osmosis membrane, Separation and Purification Technology, 204 (2018) 154-161.

[12] J. Kessler, C. Moody, Drinking water from sea water by forward osmosis, Desalination, 18 (1976) 297306.

[13] W.L. Ang, A.W. Mohammad, D. Johnson, N. Hilal, Forward osmosis research trends in desalination and wastewater treatment: A review of research trends over the past decade, Journal of Water Process Engineering, 31 (2019) 100886.

[14] J. Beh, B. Ooi, J. Lim, E. Ng, H. Mustapa, Development of high water permeability and chemically stable thin film nanocomposite (TFN) forward osmosis (FO) membrane with poly (sodium 4styrenesulfonate)(PSS)-coated zeolitic imidazolate framework-8 (ZIF-8) for produced water treatment, Journal of Water Process Engineering, 33 (2020) 101031.

[15] D. Emadzadeh, T. Matsuura, M. Ghanbari, A.F. Ismail, Hybrid forward osmosis/ultrafiltration membrane bag for water purification, Desalination, 468 (2019) 114071.

[16] Y. Huang, P. Cay-Durgun, T. Lai, P. Yu, M.L. Lind, Phenol removal from water by polyamide and $\mathrm{AgCl}$ mineralized thin-film composite forward osmosis membranes, Industrial \& Engineering Chemistry Research, 57 (2018) 7021-7029.

[17] J.E. Kim, S. Phuntsho, S.M. Ali, J.Y. Choi, H.K. Shon, Forward osmosis membrane modular configurations for osmotic dilution of seawater by forward osmosis and reverse osmosis hybrid system, Water research, 128 (2018) 183-192.

[18] C.F. Wan, Y. Cui, W.X. Gai, Z.L. Cheng, T.-S. Chung, Nanostructured Membranes for Enhanced Forward Osmosis and Pressure-Retarded Osmosis, in: Sustainable Nanoscale Engineering, Elsevier, 2020, pp. 373394.

[19] L. Deng, Q. Wang, X. An, Z. Li, Y. Hu, Towards enhanced antifouling and flux performances of thin-film composite forward osmosis membrane via constructing a sandwich-like carbon nanotubes-coated support, Desalination, 479 (2020) 114311.

[20] H. Guo, Z. Yao, J. Wang, Z. Yang, X. Ma, C.Y. Tang, Polydopamine coating on a thin film composite forward osmosis membrane for enhanced mass transport and antifouling performance, Journal of Membrane Science, 551 (2018) 234-242.

[21] W. Suwaileh, D. Johnson, S. Khodabakhshi, N. Hilal, Superior cross-linking assisted layer by layer modification of forward osmosis membranes for brackish water desalination, Desalination, 463 (2019) 112.

[22] D.J. Johnson, W.A. Suwaileh, A.W. Mohammed, N. Hilal, Osmotic's potential: An overview of draw solutes for forward osmosis, Desalination, 434 (2018) 100-120.

[23] S.M. Ali, J.E. Kim, S. Phuntsho, A. Jang, J.Y. Choi, H.K. Shon, Forward osmosis system analysis for optimum design and operating conditions, Water research, 145 (2018) 429-441.

[24] G. Gwak, B. Jung, S. Han, S. Hong, Evaluation of poly (aspartic acid sodium salt) as a draw solute for forward osmosis, Water research, 80 (2015) 294-305.

[25] M. Sakai, M. Seshimo, M. Matsukata, Hydrophilic ZSM-5 membrane for forward osmosis operation, Journal of Water Process Engineering, 32 (2019) 100864.

[26] T.-S. Chung, X. Li, R.C. Ong, Q. Ge, H. Wang, G. Han, Emerging forward osmosis (FO) technologies and challenges ahead for clean water and clean energy applications, Current Opinion in Chemical Engineering, 1 (2012) 246-257.

[27] M. Giagnorio, F. Ricceri, A. Tiraferri, Desalination of brackish groundwater and reuse of wastewater by forward osmosis coupled with nanofiltration for draw solution recovery, Water research, 153 (2019) 134-143. 
[28] G. Han, C.-Z. Liang, T.-S. Chung, M. Weber, C. Staudt, C. Maletzko, Combination of forward osmosis (FO) process with coagulation/flocculation (CF) for potential treatment of textile wastewater, Water research, 91 (2016) 361-370.

[29] W. Suwaileh, D. Johnson, D. Jones, N. Hilal, An integrated fertilizer driven forward osmosisrenewables powered membrane distillation system for brackish water desalination: A combined experimental and theoretical approach, Desalination, 471 (2019) 114126.

[30] W. Suwaileh, D. Johnson, S. Khodabakhshi, N. Hilal, Cross-linked layer by layer forward osmosis membrane for brackish water desalination, (2019).

[31] W. Suwaileh, D. Johnson, N. Hilal, Brackish water desalination for agriculture: Assessing the performance of inorganic fertilizer draw solutions, Desalination, 456 (2019) 53-63.

[32] M. Qasim, N.A. Darwish, S. Sarp, N. Hilal, Water desalination by forward (direct) osmosis phenomenon: A comprehensive review, Desalination, 374 (2015) 47-69.

[33] H.-g. Choi, A.A. Shah, S.-E. Nam, Y.-I. Park, H. Park, Thin-film composite membranes comprising ultrathin hydrophilic polydopamine interlayer with graphene oxide for forward osmosis, Desalination, 449 (2019) 41-49.

[34] R.V. Linares, Z. Li, S. Sarp, S.S. Bucs, G. Amy, J.S. Vrouwenvelder, Forward osmosis niches in seawater desalination and wastewater reuse, Water research, 66 (2014) 122-139.

[35] L. Zohrabian, N.P. Hankins, R.W. Field, Hybrid forward osmosis-membrane distillation system: Demonstration of technical feasibility, Journal of Water Process Engineering, 33 (2020) 101042.

[36] V. Nayak, M. Jyothi, R.G. Balakrishna, M. Padaki, V.Y. Zadorozhnyy, S. Kaloshkin, 4-aminophenyl sulfone (APS) as novel monomer in fabricating paper based TFC composite for forward osmosis: Selective layer optimization, Journal of Environmental Chemical Engineering, (2020) 103664.

[37] G. Blandin, A.R. Verliefde, J. Comas, I. Rodriguez-Roda, P. Le-Clech, Efficiently combining water reuse and desalination through forward osmosis-reverse osmosis (FO-RO) hybrids: a critical review, Membranes, 6 (2016) 37.

[38] X.-p. Wang, J. Hou, F.-s. Chen, X.-m. Meng, In-situ growth of metal-organic framework film on a polydopamine-modified flexible substrate for antibacterial and forward osmosis membranes, Separation and Purification Technology, 236 (2020) 116239.

[39] D. Li, Y. Yan, H. Wang, Recent advances in polymer and polymer composite membranes for reverse and forward osmosis processes, Progress in Polymer Science, 61 (2016) 104-155.

[40] N.B. Darwish, A. Alkhudhiri, H. AlRomaih, A. Alalawi, M.C. Leaper, N. Hilal, Effect of lithium chloride additive on forward osmosis membranes performance, Journal of Water Process Engineering, 33 (2020) 101049.

[41] J. Herron, Asymmetric forward osmosis membranes, in, Google Patents, 2008.

[42] W. Suwaileh, N. Pathak, H. Shon, N. Hilal, Forward osmosis membranes and processes: A comprehensive review of research trends and future outlook, Desalination, 485 (2020) 114455.

[43] S. Zhao, L. Zou, C.Y. Tang, D. Mulcahy, Recent developments in forward osmosis: opportunities and challenges, Journal of membrane science, 396 (2012) 1-21.

[44] A. Ismail, M. Padaki, N. Hilal, T. Matsuura, W. Lau, Thin film composite membrane-Recent development and future potential, Desalination, 356 (2015) 140-148.

[45] W. Ding, J. Cai, Z. Yu, Q. Wang, Z. Xu, Z. Wang, C. Gao, Fabrication of an aquaporin-based forward osmosis membrane through covalent bonding of a lipid bilayer to a microporous support, Journal of Materials Chemistry A, 3 (2015) 20118-20126.

[46] I. Ndiaye, S. Vaudreuil, T. Bounahmidi, Forward Osmosis Process: State-Of-The-Art of Membranes, Separation \& Purification Reviews, (2019) 1-21.

[47] Q. Liu, G.-R. Xu, Graphene oxide (GO) as functional material in tailoring polyamide thin film composite (PA-TFC) reverse osmosis (RO) membranes, Desalination, 394 (2016) 162-175. 
[48] T.Y. Cath, A.E. Childress, M. Elimelech, Forward osmosis: principles, applications, and recent developments, Journal of membrane science, 281 (2006) 70-87.

[49] M.R. Dalwani, Thin film composite nanofiltration membranes for extreme conditions, University of Twente, 2011.

[50] D.L. Shaffer, J.R. Werber, H. Jaramillo, S. Lin, M. Elimelech, Forward osmosis: where are we now?, Desalination, 356 (2015) 271-284.

[51] G.S. Ibrahim, A.M. Isloor, E. Yuliwati, A Review: Desalination by Forward Osmosis, in: Current Trends and Future Developments on (Bio-) Membranes, Elsevier, 2019, pp. 199-214.

[52] M. Li, V. Karanikola, X. Zhang, L. Wang, M. Elimelech, A self-standing, support-free membrane for forward osmosis with no internal concentration polarization, Environmental Science \& Technology Letters, 5 (2018) 266-271.

[53] J. Zhou, H.-L. He, F. Sun, Y. Su, H.-Y. Yu, J.-S. Gu, Structural parameters reduction in polyamide forward osmosis membranes via click modification of the polysulfone support, Colloids and Surfaces A: Physicochemical and Engineering Aspects, (2019) 124082.

[54] M. Darwish, H. Abdulrahim, A. Hassan, A. Mabrouk, A. Sharif, The forward osmosis and desalination, Desalination and Water Treatment, 57 (2016) 4269-4295.

[55] J.Y. Law, A.W. Mohammad, Employing forward osmosis technology through hybrid system configurations for the production of potable/pure water: a review, Jurnal Teknologi, 79 (2017).

[56] L. Chekli, S. Phuntsho, J.E. Kim, J. Kim, J.Y. Choi, J.-S. Choi, S. Kim, J.H. Kim, S. Hong, J. Sohn, A comprehensive review of hybrid forward osmosis systems: Performance, applications and future prospects, Journal of Membrane Science, 497 (2016) 430-449.

[57] W.L. Ang, A.W. Mohammad, D. Johnson, N. Hilal, Unlocking the application potential of forward osmosis through integrated/hybrid process, Science of The Total Environment, (2019) 136047.

[58] Y. Cai, A critical review on draw solutes development for forward osmosis, Desalination, 391 (2016) 16-29.

[59] H. Luo, Q. Wang, T.C. Zhang, T. Tao, A. Zhou, L. Chen, X. Bie, A review on the recovery methods of draw solutes in forward osmosis, Journal of Water Process Engineering, 4 (2014) 212-223.

[60] L. Li, X.-p. Liu, H.-q. Li, A review of forward osmosis membrane fouling: types, research methods and future prospects, Environmental Technology Reviews, 6 (2017) 26-46.

[61] Q. She, R. Wang, A.G. Fane, C.Y. Tang, Membrane fouling in osmotically driven membrane processes: A review, Journal of Membrane Science, 499 (2016) 201-233.

[62] Y. Chun, D. Mulcahy, L. Zou, I.S. Kim, A short review of membrane fouling in forward osmosis processes, Membranes, 7 (2017) 30.

[63] C.S. Ong, P. Goh, W. Lau, N. Misdan, A.F. Ismail, Nanomaterials for biofouling and scaling mitigation of thin film composite membrane: A review, Desalination, 393 (2016) 2-15.

[64] W. Xu, Q. Ge, Synthetic polymer materials for forward osmosis (FO) membranes and FO applications: a review, Reviews in Chemical Engineering, 35 (2019) 191-209.

[65] W. Xu, Q. Chen, Q. Ge, Recent advances in forward osmosis (FO) membrane: Chemical modifications on membranes for FO processes, Desalination, 419 (2017) 101-116.

[66] Y.-N. Wang, K. Goh, X. Li, L. Setiawan, R. Wang, Membranes and processes for forward osmosis-based desalination: Recent advances and future prospects, Desalination, 434 (2018) 81-99.

[67] Q. Chen, W. Xu, Q. Ge, Progress in Forward Osmosis Membrane Separation Process, General Chemistry, 3 (2017).

[68] W.A. Suwaileh, D.J. Johnson, S. Sarp, N. Hilal, Advances in forward osmosis membranes: altering the sub-layer structure via recent fabrication and chemical modification approaches, Desalination, 436 (2018) 176-201. 
[69] M. Amini, M. Jahanshahi, A. Rahimpour, Synthesis of novel thin film nanocomposite (TFN) forward osmosis membranes using functionalized multi-walled carbon nanotubes, Journal of membrane science, 435 (2013) 233-241.

[70] L.A. Hoover, J.D. Schiffman, M. Elimelech, Nanofibers in thin-film composite membrane support layers: Enabling expanded application of forward and pressure retarded osmosis, Desalination, 308 (2013) 73-81.

[71] N.-N. Bui, M.L. Lind, E.M. Hoek, J.R. McCutcheon, Electrospun nanofiber supported thin film composite membranes for engineered osmosis, Journal of membrane science, 385 (2011) 10-19.

[72] F.E. Ahmed, B.S. Lalia, R. Hashaikeh, A review on electrospinning for membrane fabrication: challenges and applications, Desalination, 356 (2015) 15-30.

[73] Y. Zhang, T. Mu, M. Huang, G. Chen, T. Cai, H. Chen, L. Meng, X. Luo, Nanofiber composite forward osmosis (NCFO) membranes for enhanced antibiotics rejection: Fabrication, performance, mechanism, and simulation, Journal of Membrane Science, 595 (2020) 117425.

[74] W. Lau, A. Ismail, N. Misdan, M. Kassim, A recent progress in thin film composite membrane: a review, Desalination, 287 (2012) 190-199.

[75] J.R. McCutcheon, R.L. McGinnis, M. Elimelech, Desalination by ammonia-carbon dioxide forward osmosis: influence of draw and feed solution concentrations on process performance, Journal of membrane science, 278 (2006) 114-123.

[76] Z. Yang, H. Guo, C.Y. Tang, The upper bound of thin-film composite (TFC) polyamide membranes for desalination, Journal of Membrane Science, (2019) 117297.

[77] A. Soroush, J. Barzin, M. Barikani, M. Fathizadeh, Interfacially polymerized polyamide thin film composite membranes: Preparation, characterization and performance evaluation, Desalination, 287 (2012) 310-316.

[78] A. Tiraferri, N.Y. Yip, W.A. Phillip, J.D. Schiffman, M. Elimelech, Relating performance of thin-film composite forward osmosis membranes to support layer formation and structure, Journal of Membrane Science, 367 (2011) 340-352.

[79] G. Arthanareeswaran, V.M. Starov, Effect of solvents on performance of polyethersulfone ultrafiltration membranes: Investigation of metal ion separations, Desalination, 267 (2011) 57-63.

[80] S. Madaeni, A. Taheri, Preparation of PES ultrafiltration membrane for treatment of emulsified oily wastewater: Effect of solvent and non-solvent on morphology and performance, Journal of Polymer Engineering, 29 (2009) 183-198.

[81] N. Widjojo, T.-S. Chung, M. Weber, C. Maletzko, V. Warzelhan, The role of sulphonated polymer and macrovoid-free structure in the support layer for thin-film composite (TFC) forward osmosis (FO) membranes, Journal of membrane science, 383 (2011) 214-223.

[82] J. Jegal, S.G. Min, K.H. Lee, Factors affecting the interfacial polymerization of polyamide active layers for the formation of polyamide composite membranes, Journal of applied polymer science, 86 (2002) 2781-2787.

[83] C. Klaysom, S. Hermans, A. Gahlaut, S. Van Craenenbroeck, I.F. Vankelecom, Polyamide/Polyacrylonitrile (PA/PAN) thin film composite osmosis membranes: Film optimization, characterization and performance evaluation, Journal of membrane science, 445 (2013) 25-33.

[84] J. Wei, X. Liu, C. Qiu, R. Wang, C.Y. Tang, Influence of monomer concentrations on the performance of polyamide-based thin film composite forward osmosis membranes, Journal of membrane science, 381 (2011) 110-117.

44 [85] G. Han, S. Zhang, X. Li, N. Widjojo, T.-S. Chung, Thin film composite forward osmosis membranes 45 based on polydopamine modified polysulfone substrates with enhancements in both water flux and salt 46 rejection, Chemical Engineering Science, 80 (2012) 219-231. 
[86] W. Xie, G.M. Geise, B.D. Freeman, H.-S. Lee, G. Byun, J.E. McGrath, Polyamide interfacial composite membranes prepared from $\mathrm{m}$-phenylene diamine, trimesoyl chloride and a new disulfonated diamine, Journal of membrane science, 403 (2012) 152-161.

[87] G.-Y. Chai, W.B. Krantz, Formation and characterization of polyamide membranes via interfacial polymerization, Journal of Membrane Science, 93 (1994) 175-192.

[88] B. Khorshidi, T. Thundat, B. Fleck, M. Sadrzadeh, Thin film composite polyamide membranes: parametric study on the influence of synthesis conditions, RSC Advances, 5 (2015) 54985-54997.

[89] L. Huang, J.R. McCutcheon, Impact of support layer pore size on performance of thin film composite membranes for forward osmosis, Journal of Membrane Science, 483 (2015) 25-33.

[90] L. Huang, N.-N. Bui, M.T. Meyering, T.J. Hamlin, J.R. McCutcheon, Novel hydrophilic nylon 6, 6 microfiltration membrane supported thin film composite membranes for engineered osmosis, Journal of membrane science, 437 (2013) 141-149.

[91] P.S. Singh, S. Joshi, J. Trivedi, C. Devmurari, A.P. Rao, P. Ghosh, Probing the structural variations of thin film composite RO membranes obtained by coating polyamide over polysulfone membranes of different pore dimensions, Journal of membrane science, 278 (2006) 19-25.

[92] S.J. Kwon, S.-H. Park, M.G. Shin, M.S. Park, K. Park, S. Hong, H. Park, Y.-I. Park, J.-H. Lee, Fabrication of high performance and durable forward osmosis membranes using mussel-inspired polydopaminemodified polyethylene supports, Journal of Membrane Science, 584 (2019) 89-99.

[93] J. Wei, C. Qiu, C.Y. Tang, R. Wang, A.G. Fane, Synthesis and characterization of flat-sheet thin film composite forward osmosis membranes, Journal of Membrane Science, 372 (2011) 292-302.

[94] M. Tian, C. Qiu, Y. Liao, S. Chou, R. Wang, Preparation of polyamide thin film composite forward osmosis membranes using electrospun polyvinylidene fluoride (PVDF) nanofibers as substrates, Separation and Purification Technology, 118 (2013) 727-736.

[95] Z. Zhou, J.Y. Lee, T.-S. Chung, Thin film composite forward-osmosis membranes with enhanced internal osmotic pressure for internal concentration polarization reduction, Chemical Engineering Journal, 249 (2014) 236-245.

[96] Y. Sun, L. Xue, Y. Zhang, X. Zhao, Y. Huang, X. Du, High flux polyamide thin film composite forward osmosis membranes prepared from porous substrates made of polysulfone and polyethersulfone blends, Desalination, 336 (2014) 72-79.

[97] P. Hajighahremanzadeh, M. Abbaszadeh, S.A. Mousavi, M. Soltanieh, H. Bakhshi, Polyamide/polyacrylonitrile thin film composites as forward osmosis membranes, Journal of Applied Polymer Science, 133 (2016).

[98] L. Huang, J.T. Arena, J.R. McCutcheon, Surface modified PVDF nanofiber supported thin film composite membranes for forward osmosis, Journal of Membrane Science, 499 (2016) 352-360.

[99] Y. Zhao, X. Wang, Y. Ren, D. Pei, Mesh-embedded polysulfone (PSU)/sulfonated polysulfone (sPSU) supported thin film composite membranes for forward osmosis, (2017).

[100] S. Sahebi, S. Phuntsho, Y.C. Woo, M.J. Park, L.D. Tijing, S. Hong, H.K. Shon, Effect of sulphonated polyethersulfone substrate for thin film composite forward osmosis membrane, Desalination, 389 (2016) 129-136.

[101] M.J. Park, R.R. Gonzales, A. Abdel-Wahab, S. Phuntsho, H.K. Shon, Hydrophilic polyvinyl alcohol coating on hydrophobic electrospun nanofiber membrane for high performance thin film composite forward osmosis membrane, Desalination, 426 (2018) 50-59.

[102] S. Shokrollahzadeh, S. Tajik, Fabrication of thin film composite forward osmosis membrane using electrospun polysulfone/polyacrylonitrile blend nanofibers as porous substrate, Desalination, 425 (2018) 68-76.

[103] Q.-Y. Wu, X.-Y. Xing, Y. Yu, L. Gu, Z.-K. Xu, Novel thin film composite membranes supported by cellulose triacetate porous substrates for high-performance forward osmosis, Polymer, 153 (2018) 150160. 
[104] T. Ni, Q. Ge, Highly hydrophilic thin-film composition forward osmosis (FO) membranes functionalized with aniline sulfonate/bisulfonate for desalination, Journal of Membrane Science, 564 (2018) 732-741.

[105] M. Shibuya, M.J. Park, S. Lim, S. Phuntsho, H. Matsuyama, H.K. Shon, Novel CA/PVDF nanofiber supports strategically designed via coaxial electrospinning for high performance thin-film composite forward osmosis membranes for desalination, Desalination, 445 (2018) 63-74.

[106] K. Zheng, S. Zhou, X. Zhou, High-performance thin-film composite forward osmosis membrane fabricated on low-cost PVB/PVC substrate, New Journal of Chemistry, 42 (2018) 13382-13392.

[107] Y.-H. Chiao, A. Sengupta, S.-T. Chen, W.-S. Hung, J.-Y. Lai, L. Upadhyaya, X. Qian, S.R. Wickramasinghe, Novel thin-film composite forward osmosis membrane using polyethylenimine and its impact on membrane performance, Separation Science and Technology, (2019) 1-11.

[108] H.-E. Kwon, S.J. Kwon, S.-J. Park, M.G. Shin, S.-H. Park, M.S. Park, H. Park, J.-H. Lee, High performance polyacrylonitrile-supported forward osmosis membranes prepared via aromatic solvent-based interfacial polymerization, Separation and Purification Technology, 212 (2019) 449-457.

[109] M. Zheng, X. Zhao, S. Xu, D. Lu, Ultrathin Support-Free Membrane with High Water Flux for Forward Osmosis Desalination, Water, Air, \& Soil Pollution, 230 (2019) 138.

[110] K. Zheng, S. Zhou, Fabrication of a novel cyanoethyl cellulose substrate for thin-film composite forward osmosis membrane, Blue Green Systems, 1 (2019) 18-32.

[111] R. Thür, M. Corvilain, C. Klaysom, Y. Hartanto, I.F. Vankelecom, Tuning the selectivity of thin film composite forward osmosis membranes: effect of co-solvent and different interfacial polymerization synthesis routes, Separation and Purification Technology, (2019).

[112] M. Rastgar, A. Shakeri, H. Salehi, Study of polyamide thin film characteristics impact on permeability/selectivity performance and fouling behavior of forward osmosis membrane, Environmental Science and Pollution Research, 26 (2019) 1181-1191.

[113] W. Lee, P. Goh, W. Lau, C. Ong, A. Ismail, Antifouling zwitterion embedded forward osmosis thin film composite membrane for highly concentrated oily wastewater treatment, Separation and Purification Technology, 214 (2019) 40-50.

[114] X. Hao, S. Gao, J. Tian, Y. Sun, F. Cui, C.Y. Tang, Calcium-carboxyl intrabridging during interfacial polymerization: a novel strategy to improve antifouling performance of thin film composite membranes, Environmental science \& technology, 53 (2019) 4371-4379.

[115] J. Ma, T. Xiao, N. Long, X. Yang, The Role of Polyvinyl Butyral Additive in Forming Desirable Pore Structure for Thin Film Composite Forward Osmosis Membrane, Separation and Purification Technology, (2020) 116798.

[116] L. Shen, W.-s. Hung, J. Zuo, L. Tian, M. Yi, C. Ding, Y. Wang, Effect of ultrasonication parameters on forward osmosis performance of thin film composite polyamide membranes prepared with ultrasoundassisted interfacial polymerization, Journal of Membrane Science, (2020) 117834.

[117] C.F. Wan, T. Yang, G.G. Lipscomb, D.J. Stookey, T.-S. Chung, Design and fabrication of hollow fiber membrane modules, Journal of Membrane Science, 538 (2017) 96-107.

[118] S.-P. Sun, T.-S. Chung, Outer-selective pressure-retarded osmosis hollow fiber membranes from vacuum-assisted interfacial polymerization for osmotic power generation, Environmental science \& technology, 47 (2013) 13167-13174.

[119] R. Wang, L. Shi, C.Y. Tang, S. Chou, C. Qiu, A.G. Fane, Characterization of novel forward osmosis hollow fiber membranes, Journal of membrane science, 355 (2010) 158-167.

[120] P. Zhong, X. Fu, T.-S. Chung, M. Weber, C. Maletzko, Development of thin-film composite forward osmosis hollow fiber membranes using direct sulfonated polyphenylenesulfone (sPPSU) as membrane substrates, Environmental science \& technology, 47 (2013) 7430-7436.

[121] L. Luo, P. Wang, S. Zhang, G. Han, T.-S. Chung, Novel thin-film composite tri-bore hollow fiber membrane fabrication for forward osmosis, Journal of Membrane Science, 461 (2014) 28-38. 
[122] N. Peng, N. Widjojo, P. Sukitpaneenit, M.M. Teoh, G.G. Lipscomb, T.-S. Chung, J.-Y. Lai, Evolution of polymeric hollow fibers as sustainable technologies: Past, present, and future, Progress in Polymer Science, 37 (2012) 1401-1424.

[123] L. Shi, S. Chou, R. Wang, W. Fang, C. Tang, A. Fane, Effect of substrate structure on the performance of thin-film composite forward osmosis hollow fiber membranes, Journal of membrane science, 382 (2011) 116-123.

[124] P. Sukitpaneenit, T.-S. Chung, High performance thin-film composite forward osmosis hollow fiber membranes with macrovoid-free and highly porous structure for sustainable water production, Environmental science \& technology, 46 (2012) 7358-7365.

[125] S. Chou, L. Shi, R. Wang, C.Y. Tang, C. Qiu, A.G. Fane, Characteristics and potential applications of a novel forward osmosis hollow fiber membrane, Desalination, 261 (2010) 365-372.

[126] S. Lim, V.H. Tran, N. Akther, S. Phuntsho, H.K. Shon, Defect-free outer-selective hollow fiber thinfilm composite membranes for forward osmosis applications, Journal of Membrane Science, 586 (2019) 281-291.

[127] R.W. Baker, Membrane technology and applications. 2004, England: John Wiley and Sons, (2007). [128] C.Y. Tang, Q. She, W.C. Lay, R. Wang, A.G. Fane, Coupled effects of internal concentration polarization and fouling on flux behavior of forward osmosis membranes during humic acid filtration, Journal of membrane science, 354 (2010) 123-133.

[129] D.F. Sanders, Z.P. Smith, R. Guo, L.M. Robeson, J.E. McGrath, D.R. Paul, B.D. Freeman, Energyefficient polymeric gas separation membranes for a sustainable future: A review, Polymer, 54 (2013) 47294761.

[130] P. Wang, L. Luo, T.-S. Chung, Tri-bore ultra-filtration hollow fiber membranes with a novel triangleshape outer geometry, Journal of Membrane Science, 452 (2014) 212-218.

[131] J. Ren, J.R. McCutcheon, Polyacrylonitrile supported thin film composite hollow fiber membranes for forward osmosis, Desalination, 372 (2015) 67-74.

[132] M. Shibuya, M. Yasukawa, S. Mishima, Y. Tanaka, T. Takahashi, H. Matsuyama, A thin-film composite-hollow fiber forward osmosis membrane with a polyketone hollow fiber membrane as a support, Desalination, 402 (2017) 33-41.

[133] M. Yasukawa, S. Mishima, M. Shibuya, D. Saeki, T. Takahashi, T. Miyoshi, H. Matsuyama, Preparation of a forward osmosis membrane using a highly porous polyketone microfiltration membrane as a novel support, Journal of Membrane Science, 487 (2015) 51-59.

[134] Q. Jia, H. Han, L. Wang, B. Liu, H. Yang, J. Shen, Effects of CTAC micelles on the molecular structures and separation performance of thin-film composite (TFC) membranes in forward osmosis processes, Desalination, 340 (2014) 30-41.

[135] W. Lee, P.K. Kang, A.S. Kim, S. Lee, Impact of surface porosity on water flux and structural parameter in forward osmosis, Desalination, 439 (2018) 46-57.

[136] C.H. Tan, H.Y. Ng, Modified models to predict flux behavior in forward osmosis in consideration of external and internal concentration polarizations, Journal of Membrane science, 324 (2008) 209-219.

[137] C. Suh, S. Lee, Modeling reverse draw solute flux in forward osmosis with external concentration polarization in both sides of the draw and feed solution, Journal of membrane science, 427 (2013) 365374.

[138] N. Niksefat, M. Jahanshahi, A. Rahimpour, The effect of SiO 2 nanoparticles on morphology and performance of thin film composite membranes for forward osmosis application, Desalination, 343 (2014) 140-146.

[139] A.F. Faria, C. Liu, M. Xie, F. Perreault, L.D. Nghiem, J. Ma, M. Elimelech, Thin-film composite forward osmosis membranes functionalized with graphene oxide-silver nanocomposites for biofouling control, Journal of Membrane Science, 525 (2017) 146-156. 
[140] D. Emadzadeh, W. Lau, T. Matsuura, N. Hilal, A. Ismail, The potential of thin film nanocomposite membrane in reducing organic fouling in forward osmosis process, Desalination, 348 (2014) 82-88.

[141] A. Zirehpour, A. Rahimpour, A. Arabi Shamsabadi, M. Sharifian Gh, M. Soroush, Mitigation of thinfilm composite membrane biofouling via immobilizing nano-sized biocidal reservoirs in the membrane active layer, Environmental science \& technology, 51 (2017) 5511-5522.

[142] A. Rahimpour, S.F. Seyedpour, S. Aghapour Aktij, M. Dadashi Firouzjaei, A. Zirehpour, A. Arabi Shamsabadi, S. Khoshhal Salestan, M. Jabbari, M. Soroush, Simultaneous improvement of antimicrobial, antifouling, and transport properties of forward osmosis membranes with immobilized highly-compatible polyrhodanine nanoparticles, Environmental science \& technology, 52 (2018) 5246-5258.

[143] D. Emadzadeh, W.J. Lau, T. Matsuura, M. Rahbari-Sisakht, A. Ismail, A novel thin film composite forward osmosis membrane prepared from PSf-TiO 2 nanocomposite substrate for water desalination, Chemical Engineering Journal, 237 (2014) 70-80.

[144] D. Emadzadeh, W. Lau, M. Rahbari-Sisakht, H. Ilbeygi, D. Rana, T. Matsuura, A. Ismail, Synthesis, modification and optimization of titanate nanotubes-polyamide thin film nanocomposite (TFN) membrane for forward osmosis (FO) application, Chemical Engineering Journal, 281 (2015) 243-251.

[145] W. Lau, S. Gray, T. Matsuura, D. Emadzadeh, J.P. Chen, A. Ismail, A review on polyamide thin film nanocomposite (TFN) membranes: history, applications, challenges and approaches, water research, 80 (2015) 306-324.

[146] M. Rastgar, A. Shakeri, A. Bozorg, H. Salehi, V. Saadattalab, Impact of nanoparticles surface characteristics on pore structure and performance of forward osmosis membranes, Desalination, (2017). [147] M. Tian, Y.-N. Wang, R. Wang, A.G. Fane, Synthesis and characterization of thin film nanocomposite forward osmosis membranes supported by silica nanoparticle incorporated nanofibrous substrate, Desalination, 401 (2017) 142-150.

[148] N. Ma, J. Wei, R. Liao, C.Y. Tang, Zeolite-polyamide thin film nanocomposite membranes: towards enhanced performance for forward osmosis, Journal of membrane science, 405 (2012) 149-157.

[149] M. Rezaei-DashtArzhandi, M. Sarrafzadeh, P. Goh, W. Lau, A. Ismail, M. Mohamed, Development of novel thin film nanocomposite forward osmosis membranes containing halloysite/graphitic carbon nitride nanoparticles towards enhanced desalination performance, Desalination, 447 (2018) 18-28.

[150] A. Zirehpour, A. Rahimpour, M. Ulbricht, Nano-sized metal organic framework to improve the structural properties and desalination performance of thin film composite forward osmosis membrane, Journal of Membrane Science, 531 (2017) 59-67.

[151] A. Shakeri, H. Salehi, F. Ghorbani, M. Amini, H. Naslhajian, Polyoxometalate based thin film nanocomposite forward osmosis membrane: Superhydrophilic, anti-fouling, and high water permeable, Journal of colloid and interface science, 536 (2019) 328-338.

[152] A.L. Ohland, V.M.M. Salim, C.P. Borges, Nanocomposite membranes for osmotic processes: Incorporation of functionalized hydroxyapatite in porous substrate and in selective layer, Desalination, 463 (2019) 23-31.

[153] N. Ma, J. Wei, S. Qi, Y. Zhao, Y. Gao, C.Y. Tang, Nanocomposite substrates for controlling internal concentration polarization in forward osmosis membranes, Journal of membrane science, 441 (2013) 5462.

[154] M.J. Park, S. Phuntsho, T. He, G.M. Nisola, L.D. Tijing, X.-M. Li, G. Chen, W.-J. Chung, H.K. Shon, Graphene oxide incorporated polysulfone substrate for the fabrication of flat-sheet thin-film composite forward osmosis membranes, Journal of Membrane Science, 493 (2015) 496-507.

[155] X. Liu, H.Y. Ng, Fabrication of layered silica-polysulfone mixed matrix substrate membrane for enhancing performance of thin-film composite forward osmosis membrane, Journal of Membrane Science, 481 (2015) 148-163. 
[156] M. Tian, Y.-N. Wang, R. Wang, Synthesis and characterization of novel high-performance thin film nanocomposite (TFN) FO membranes with nanofibrous substrate reinforced by functionalized carbon nanotubes, Desalination, 370 (2015) 79-86.

[157] M. Ghanbari, D. Emadzadeh, W. Lau, H. Riazi, D. Almasi, A. Ismail, Minimizing structural parameter of thin film composite forward osmosis membranes using polysulfone/halloysite nanotubes as membrane substrates, Desalination, 377 (2016) 152-162.

[158] P. Lu, S. Liang, L. Qiu, Y. Gao, Q. Wang, Thin film nanocomposite forward osmosis membranes based on layered double hydroxide nanoparticles blended substrates, Journal of Membrane Science, 504 (2016) 196-205.

[159] P. Lu, S. Liang, T. Zhou, X. Mei, Y. Zhang, C. Zhang, A. Umar, Q. Wang, Layered double hydroxide/graphene oxide hybrid incorporated polysulfone substrate for thin-film nanocomposite forward osmosis membranes, RSC Advances, 6 (2016) 56599-56609.

[160] S. Lim, M.J. Park, S. Phuntsho, L.D. Tijing, G.M. Nisola, W.-G. Shim, W.-J. Chung, H.K. Shon, Duallayered nanocomposite substrate membrane based on polysulfone/graphene oxide for mitigating internal concentration polarization in forward osmosis, Polymer, 110 (2017) 36-48.

[161] Y.-H. Pan, Q.-Y. Zhao, L. Gu, Q.-Y. Wu, Thin film nanocomposite membranes based on imologite nanotubes blended substrates for forward osmosis desalination, Desalination, 421 (2017) 160-168.

[162] D. Ma, G. Han, S.B. Peh, S.B. Chen, Water-stable metal-organic framework UiO-66 for performance enhancement of forward osmosis membranes, Industrial \& Engineering Chemistry Research, 56 (2017) 12773-12782.

[163] L. Shen, S. Xiong, Y. Wang, Graphene oxide incorporated thin-film composite membranes for forward osmosis applications, Chemical Engineering Science, 143 (2016) 194-205.

[164] D. Emadzadeh, M. Ghanbari, W. Lau, M. Rahbari-Sisakht, T. Matsuura, A. Ismail, B. Kruczek, Solvothermal synthesis of nanoporous TiO2: the impact on thin-film composite membranes for engineered osmosis application, Nanotechnology, 27 (2016) 345702.

[165] D. Ma, S.B. Peh, G. Han, S.B. Chen, Thin-film nanocomposite (TFN) membranes incorporated with super-hydrophilic metal-organic framework (MOF) UiO-66: toward enhancement of water flux and salt rejection, ACS applied materials \& interfaces, 9 (2017) 7523-7534.

[166] S. Shokrgozar Eslah, S. Shokrollahzadeh, O. Moini Jazani, A. Samimi, Forward osmosis water desalination: Fabrication of graphene oxide-polyamide/polysulfone thin-film nanocomposite membrane with high water flux and low reverse salt diffusion, Separation Science and Technology, 53 (2018) 573583.

[167] S.F. Seyedpour, A. Rahimpour, A.A. Shamsabadi, M. Soroush, Improved performance and antifouling properties of thin-film composite polyamide membranes modified with nano-sized bactericidal graphene quantum dots for forward osmosis, Chemical Engineering Research and Design, 139 (2018) 321-334.

[168] M.G.N. Perera, Y.R. Galagedara, Y. Ren, M. Jayaweera, Y. Zhao, R. Weerasooriya, Fabrication of fullerenol-incorporated thin-film nanocomposite forward osmosis membranes for improved desalination performances, Journal of Polymer Research, 25 (2018) 199.

[169] R.R. Darabi, M. Jahanshahi, M. Peyravi, A support assisted by photocatalytic Fe304/ZnO nanocomposite for thin-film forward osmosis membrane, Chemical Engineering Research and Design, 133 (2018) 11-25.

[170] R.R. Darabi, M. Peyravi, M. Jahanshahi, Modified forward osmosis membranes by two aminofunctionalized ZnO nanoparticles: A comparative study, Chemical Engineering Research and Design, 145 (2019) 85-98.

[171] S.-J. Shi, Y.-H. Pan, S.-F. Wang, Z.-W. Dai, L. Gu, Q.-Y. Wu, Aluminosilicate Nanotubes Embedded Polyamide Thin Film Nanocomposite Forward Osmosis Membranes with Simultaneous Enhancement of Water Permeability and Selectivity, Polymers, 11 (2019) 879. 
[172] A. Shakeri, H. Mighani, N. Salari, H. Salehi, Surface modification of forward osmosis membrane using polyoxometalate based open frameworks for hydrophilicity and water flux improvement, Journal of Water Process Engineering, 29 (2019) 100762.

[173] N. Akther, S. Lim, V.H. Tran, S. Phuntsho, Y. Yang, T.-H. Bae, N. Ghaffour, H.K. Shon, The effect of Schiff base network on the separation performance of thin film nanocomposite forward osmosis membranes, Separation and Purification Technology, 217 (2019) 284-293.

[174] R. Dai, X. Zhang, M. Liu, Z. Wu, Z. Wang, Porous metal organic framework CuBDC nanosheet incorporated thin-film nanocomposite membrane for high-performance forward osmosis, Journal of membrane science, 573 (2019) 46-54.

[175] A. Shakeri, H. Salehi, S.R. Razavi, S.M.M. Babaheydari, Blue lemon@ quaternary graphene oxide open frameworks: As a novel nanostructure for performance enhancement of thin film nanocomposite forward osmosis membrane, Chemical Engineering Research and Design, 148 (2019) 451-459.

[176] A. Shakeri, R. Razavi, H. Salehi, M. Fallahi, T. Eghbalazar, Thin film nanocomposite forward osmosis membrane embedded with amine-functionalized ordered mesoporous silica, Applied Surface Science, 481 (2019) 811-818.

[177] M. Shan, H. Kang, Z. Xu, N. Li, M. Jing, Y. Hu, K. Teng, X. Qian, J. Shi, L. Liu, Decreased cross-linking in interfacial polymerization and heteromorphic support between nanoparticles: Towards high-water and low-solute flux of hybrid forward osmosis membrane, Journal of colloid and interface science, 548 (2019) 170-183.

[178] M. Bagherzadeh, A. Bayrami, M. Amini, Enhancing forward osmosis (FO) performance of polyethersulfone/polyamide (PES/PA) thin-film composite membrane via the incorporation of GQDs@ UiO-66-NH2 particles, Journal of Water Process Engineering, 33 (2020) 101107.

[179] W. Ding, Y. Li, M. Bao, J. Zhang, C. Zhang, J. Lu, Highly permeable and stable forward osmosis (FO) membrane based on the incorporation of $\mathrm{Al} 2 \mathrm{O} 3$ nanoparticles into both substrate and polyamide active layer, RSC Advances, 7 (2017) 40311-40320.

[180] W. Fang, R. Wang, S. Chou, L. Setiawan, A.G. Fane, Composite forward osmosis hollow fiber membranes: Integration of RO-and NF-like selective layers to enhance membrane properties of antiscaling and anti-internal concentration polarization, Journal of membrane science, 394 (2012) 140-150. [181] C.Y. Tang, Q. She, W.C. Lay, R. Wang, R. Field, A.G. Fane, Modeling double-skinned FO membranes, Desalination, 283 (2011) 178-186.

[182] K.Y. Wang, R.C. Ong, T.-S. Chung, Double-skinned forward osmosis membranes for reducing internal concentration polarization within the porous sublayer, Industrial \& Engineering Chemistry Research, 49 (2010) 4824-4831.

[183] S. Zhang, K.Y. Wang, T.-S. Chung, H. Chen, Y. Jean, G. Amy, Well-constructed cellulose acetate membranes for forward osmosis: minimized internal concentration polarization with an ultra-thin selective layer, Journal of Membrane Science, 360 (2010) 522-535.

[184] G. Han, Z.L. Cheng, T.-S. Chung, Thin-film composite (TFC) hollow fiber membrane with doublepolyamide active layers for internal concentration polarization and fouling mitigation in osmotic processes, Journal of Membrane Science, 523 (2017) 497-504.

[185] L. Li, M. Chen, Y. Dong, X. Dong, S. Cerneaux, S. Hampshire, J. Cao, L. Zhu, Z. Zhu, J. Liu, A low-cost alumina-mullite composite hollow fiber ceramic membrane fabricated via phase-inversion and sintering method, Journal of the European Ceramic Society, 36 (2016) 2057-2066.

[186] P.H. Duong, T.-S. Chung, S. Wei, L. Irish, Highly permeable double-skinned forward osmosis membranes for anti-fouling in the emulsified oil-water separation process, Environmental science \& technology, 48 (2014) 4537-4545.

[187] X. Song, L. Wang, C.Y. Tang, Z. Wang, C. Gao, Fabrication of carbon nanotubes incorporated doubleskinned thin film nanocomposite membranes for enhanced separation performance and antifouling capability in forward osmosis process, Desalination, 369 (2015) 1-9. 
[188] C.S. Ong, B. Al-anzi, W.J. Lau, P.S. Goh, G.S. Lai, A.F. Ismail, Y.S. Ong, Anti-Fouling Double-Skinned Forward Osmosis Membrane with Zwitterionic Brush for Oily Wastewater Treatment, Scientific reports, 7 (2017) 6904.

[189] X. Zhang, M. Xie, Z. Yang, H.-C. Wu, C. Fang, L. Bai, L.-F. Fang, T. Yoshioka, H. Matsuyama, Antifouling Double-Skinned Forward Osmosis Membranes by Constructing Zwitterionic Brush-Decorated MWCNT Ultrathin Films, ACS applied materials \& interfaces, (2019).

[190] A.M. Awad, R. Jalab, J. Minier-Matar, S. Adham, M.S. Nasser, S. Judd, The status of forward osmosis technology implementation, Desalination, 461 (2019) 10-21.

[191] J. Ren, J.R. McCutcheon, A new commercial thin film composite membrane for forward osmosis, Desalination, 343 (2014) 187-193.

[192] J. Ren, J.R. McCutcheon, A new commercial biomimetic hollow fiber membrane for forward osmosis, Desalination, 442 (2018) 44-50.

[193] M. Shibuya, M. Yasukawa, T. Takahashi, T. Miyoshi, M. Higa, H. Matsuyama, Effect of operating conditions on osmotic-driven membrane performances of cellulose triacetate forward osmosis hollow fiber membrane, Desalination, 362 (2015) 34-42.

[194] A. Ebadi Amooghin, S. Mashhadikhan, H. Sanaeepur, A. Moghadassi, T. Matsuura, S. Ramakrishna, Substantial breakthroughs on function-led design of advanced materials used in mixed matrix membranes 18 (MMMs): A new horizon for efficient CO2 separation, PROGRESS IN MATERIALS SCIENCE, 102 (2019) 22219295.

20

21

\section{ROMAN LETTERS}

$\begin{array}{ll}A & \text { water permeability coefficient }(\mathrm{LMH} / \mathrm{bar}, \mathrm{m} / \mathrm{s} \mathrm{pa}) \\ B & \text { solute permeability coefficient }(\mathrm{LMH}, \mathrm{m} / \mathrm{s}) \\ C & \text { Solute concentration }(\mathrm{mol} / \mathrm{L}) \\ J_{S} & \text { Reverse salt flux }(\mathrm{gMH}) \\ J_{S} / J_{v} & \text { specific reverse salt flux }(\mathrm{g} / \mathrm{L}) \\ J_{v} & \text { water flux }(\mathrm{LMH}) \\ l & \text { Thickness of the substrate layer }(\mu \mathrm{m}) \\ n & \text { The van't Hoff coefficient } \\ P & \text { Hydraulic pressure } \\ R & \text { Rejection rate or Universal gas constant } \\ S & \text { Structural parameter }(\mathrm{m}, \mathrm{mm}, \mu \mathrm{m})\end{array}$

22

\section{GREEK LETTERS}

$\begin{array}{ll}\varepsilon & \text { Porosity of the substrate layer } \\ \pi & \text { Osmotic pressure (bar) } \\ \tau & \text { Tortuosity }\end{array}$




\section{SUBSCRIPTS}

$\begin{array}{ll}\text { draw } & \text { Draw active layer } \\ \text { feed } & \text { Feed active layer } \\ s / f & \text { the interface between the substrate and the feed active layer } \\ \text { support } & \text { substrate layer }\end{array}$

ABBREVIATIONS

AAPTS

AL-DS

AL-FS

ANTs

CA

CAB

CEC

CFIC

CMPs

CNTs

COFs

CTA

CTAC

CTFs

DI water

DPE

DSA-2Na

ECP

FO

g-C3N4

gMH

$\mathrm{gMH} / \mathrm{bar}$

$\mathrm{GO}$

GQDs

Hapf

$\mathrm{HF}$

HNTs

HTI

ICIC

ICP

INTs

IP

IPA
N-(2-Aminoethyl)-3-aminopropyltrimethoxysilane

Active layer facing the draw solution mode; also called as PRO mode Active layer facing the feed solution mode; also called as FO mode aluminosilicate nanotubes

cellulose acetate

Cellulose acetate butyrate

Cyanoethyl Cellulose

5-chloroformyloxy-isophthaloylchloride

Conjugated microporous polymers

carbon nanotubes

Covalent organic frameworks

Cellulose triacetate

Cetyltrimethylammonium chloride

Covalent triazine based frameworks

Deionized water

Polydopamine-modified polyethylene

2,5-disulfoaniline disodium salt

External concentration polarization

Forward osmosis

Graphite-like carbon nitrite

Grams per square meter per hour

Grams per square meter per hour per bar

Graphene oxide

graphene quantum dots

hydroxyapatite particles

Hollow fiber

Halloysite nanotubes HNTs

Hydration Technologies Inc

5-isocyanato-isophthaloyl chloride

Internal concentration polarization

Imogolite nanotubes

Interfacial polymerization

Isopropyl alcohol 


\begin{tabular}{|c|c|}
\hline IPC & Isophthaloyl chloride \\
\hline LDH & Layered double hydroxide \\
\hline $\mathrm{LiCl}$ & Lithium chloride \\
\hline LMH & Liters per square meter per hour \\
\hline $\mathrm{LMH} / \mathrm{bar}$ & Liters per square meter per hour per bar \\
\hline MOF & Metal-organic framework \\
\hline MOPs & Metal organic polyhedras \\
\hline MPD & M-Phenylenediamine \\
\hline MWCNT/PSBMA & Zwitterionic brush-decorated, multiwalled carbon nanotube \\
\hline $\mathrm{NF}$ & Nanofiltration \\
\hline $\mathrm{NH}_{2}$ - TNTs & Amino-functionalized titanate nanotubes \\
\hline NMP & N-Methyl-2-pyrrolidone \\
\hline OCNTs & Oxidized carbon nanotubes \\
\hline PA & Polyamide \\
\hline PAFs & Porous aromatic frameworks \\
\hline PAI & Poly (amide-imide) \\
\hline PAN & Polyacrylonitrile \\
\hline PBI & Polybenzimidazole \\
\hline PCPs & Porous coordination polymers \\
\hline PDA & Polydopamine \\
\hline PE & Polyethylene \\
\hline$P E G$ & Polyethylene glycol \\
\hline PEI & Polyetherimide or Polyethyleneimine \\
\hline PES & Polyethersulfone \\
\hline PIP & Piperazine \\
\hline POF & Porous organic frameworks \\
\hline POM-OFs & Polyoxometalate-based open frameworks \\
\hline PPD & P-phenylene diamine \\
\hline PSF & Polysulfone \\
\hline PVA & Polyvinyl alcohol \\
\hline PVB & Poly (vinyl butyral) \\
\hline PVC & Poly (vinyl chloride) \\
\hline PVDF & Polyvinylidene fluoride \\
\hline RO & Reverse osmosis \\
\hline SBA-15-NH2 & mesoporous silica modified with amine \\
\hline SNW-1 & Schiff base network-1 \\
\hline sPEEK & Sulfonated polyetheretherketone \\
\hline SPPO & Sulfonated poly (phenylene oxide) \\
\hline sPPSU & Sulfonated poly phenylene sulfone \\
\hline sPSF & Sulfonated polysulfone \\
\hline
\end{tabular}




$\begin{array}{ll}\text { SWCNTs } & \text { Single wall carbon nanotubes } \\ \text { SWo } & \text { Spiral wound } \\ \text { TEOA } & \text { Triethanolamine } \\ \text { TFC } & \text { Thin film composite } \\ \text { TFC-A } & \text { TFC with polyamide active layer } \\ \text { TFC-D } & \text { Double-skinned TFC } \\ \text { TFC-N } & \text { Thin film nanocomposites } \\ \text { TMC } & \text { Trimesoyl Chloride } \\ \text { TNTs } & \text { Titanate nanotubes } \\ \text { UiO-66 } & \text { zirconium (IV)-carboxylate metal-organic framework } \\ \text { ZIF } & \text { Zeolitic imidazolate framework } \\ \text { ZLD } & \text { Zero liquid discharge } \\ \text { ZNPs } & \text { Zinc oxide nanoparticles } \\ \text { ZSCSNPs } & \text { ZnO-SiO2 core-shell nanoparticles }\end{array}$


1- A general performance curve for TFC-FO membranes was generated.

2- Active layer modified TFNs and double-skinned membranes possess very high potential for FO.

3- More researches should be performed on the less considered conditions of FO. 


\title{
Current status and challenges of fabricating thin film composite forward osmosis membrane: A comprehensive roadmap
}

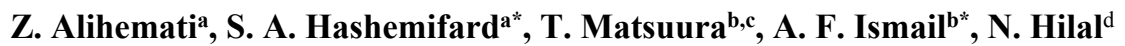 \\ ${ }^{a}$ Sustainable Membrane Technology Research Group (SMTRG), Faculty of Petroleum, Gas and Petrochemical \\ Engineering(FPGPE), Persian Gulf University (PGU), P.O. Box 75169-13798, Bushehr, Iran \\ ${ }^{b}$ Advanced Membrane Technology Research Centre (AMTEC), Universiti Teknologi Malaysia, 81310 UTM Skudai, \\ Johor Darul Ta'zim, Malaysia \\ ${ }^{c}$ Department of Chemical and Biological Engineering, University of Ottawa, 161 Louis Pasteur St., Ottawa, ON, \\ CanadaK1N 6 N5 \\ ${ }^{d}$ Centre for Water Advanced Technologies and Environmental Research (CWATER), Swansea University College of \\ Engineering, Swansea, United Kingdom
}

\begin{abstract}
Recently, forward osmosis (FO) has attracted a great deal of attention in desalination and wastewater treatment. Nevertheless, there are several critical challenges such as the need for new advances in designing membranes that must be met to enhance the water flux in FO processes, control the reverse salt flux, concentration polarization and fouling. Therefore, designing a suitable membrane with a high-water flux, low reverse salt flux, low fouling, and controlled concentration polarization seems to be essential. Thin film composite (TFC) membranes are the most widely used membranes in the FO field. Extensive research has been performed to fabricate and design high performance TFC membranes which can be exclusively used in FO processes. This paper aims to review three types of TFC membranes i.e. TFC's with polyamide active layer (TFC-A), thin film nanocomposites (TFC-N) and double-skinned TFC membranes (TFC-D) in flat sheet and hollow fiber configuration. Finally, an attempt is made to generate a general performance curve based on the water flux and reverse salt flux of these three TFC FO types and the future direction of the R and D on the FO membrane are discussed.
\end{abstract}

Key words: Forward osmosis; Water treatment; Desalination; Thin film composite membrane; Thin film nanocomposite membrane 


\title{
2 Table of Contents
}

\author{
3 1. Introduction \\ 4 2. Thin film composite membrane (TFC) \\ $5 \quad 2.1$. Synthesizing porous substrates \\ 6 2.2. Synthesizing active layers \\ 7 2.3. Performance of TFC-A flat sheet membranes \\ 8 2.3.1. Effect of MPD and TMC concentration on active layer \\ 9 2.3.2. PSF as substrate modified with polydopamine \\ 2.3.3. Polyacrylonitrile (PAN) as substrate \\ 2.3.4. Nylon 6.6 as substrate \\ 2.3.5. Polyethylene as a substrate \\ 2.4. Performance of TFC-A hollow fiber membranes \\ 2.4.1. PES as substrate \\ 2.4.2. Polyphenylene sulfone as substrate \\ 2.4.3. Matrimid as substrate \\ 2.4.4. PAN as substrate \\ 2.4.5. Polyketone as substrate \\ 2.5. Issues and Implications of TFC-A membranes \\ 2.6. Performance of TFC-N membranes \\ 2.6.1 TFC-N with modified substrate \\ 2.6.2. TFC-N with modified active layer \\ 2.6.3.TFC-N with modified both substrate and active layer concurrently \\ 2.8. Performance of TFC-D membranes \\ 2.8.1. Inner active layer RO polyamide and outer skin layer NF polyethyleneimine \\ 2.8.2 Both skin layers from polyamide \\ 3. Commercial FO membranes \\ 3.1. HTI membrane \\ 3.2. Aquaporin $\mathrm{A} / \mathrm{S}$ membrane \\ 33 \\ 4. Proposing roadmap, concluding remarks and future directions \\ 5.Refrences
}

34 


\section{Introduction}

Today, supplying adequate freshwater is crucial for human life and survival of industries. Considering the progressive growth of population and the limitation of the world freshwater reserves, rapid and economical desalination of saline/brackish water and treatment of wastewater have become increasingly important to provide clean water for different purposes [1-4]. Currently, reverse osmosis (RO) has a wide range of applications in water treatment processes due to its superiority over the other conventional methods [5-8]. Nevertheless, the energy consumption of RO is still high despite the remarkable progresses and many efforts made during the past several decades to reduce it, due primarily to the intrinsic thermodynamic constraints of the membrane desalination process [6]. Hence, the minimum amount of energy required for complete separation is at least equal to or greater than the free enthalpy of mixing [9]. Furthermore, extensive fouling and high retentate concentration are considered as the serious challenges of RO plants [10]. Recently, a novel membrane process known as forward osmosis (FO) has been proposed with the aim of saline water desalination as well as wastewater treatment [11-17]. FO applies osmotic pressure differences as a driving force to induce a net flow of water across the membrane from a feed solution (low osmotic pressure) to a draw solution (high osmotic pressure)[18-21]. Although FO process has several advantages over the conventional methods, this method also suffers from various challenges and disadvantages at present. The advantages of FO include its low energy consumption to transfer water through a semi-permeable membrane and relatively low fouling tendency [13, 22-25]. However, an additional process is required to prepare the final product, i.e. pure water, since the product cannot be consumed directly as fresh water [26-29]. The absence of properly designed FO membrane and inexpensive draw solution are considered as the other FO challenges [26, 29-36]. There are two types of membranes for FO: a) membranes originally made for RO and nanofiltration (NF); b) membranes specifically fabricated for FO [37]. An ideal FO membrane should consist of an active layer of high water permeability and low reverse solute diffusion [38] (representing the amount of salt that migrates from the draw solution towards the feed [10]) and a high flux substrate. Also, the membrane is expected to be chemically and mechanically stable showing less tendency to fouling and concentration polarization [39, 40]. Among the polymer materials that are used for synthesizing FO membranes, recent studies have focused on materials such as cellulose derivatives, polyamide (PA), polyelectrolyte, and polybenzimidazole (PBI). Cellulose derivatives such as cellulose triacetate (CTA) membranes are fabricated as flat sheet and hollow fibers via the phase inversion process followed by heat treatment [39]. Basically, they are integrally skinned asymmetric membranes. In particular, CTA membranes have been already commercialized by HTI Co. [41]. The thickness of these CTA membranes is less than the standard RO membranes [42]. The embedded polyester mesh is applied as a mechanical support for the CTA HTI membranes. Although, they exhibit higher water flux and salt rejection compared to the commercial RO membranes in FO process, the water fluxes are still far lower than the desirable values [43]. Moreover, the membranes made of cellulose derivatives suffer from the limited resistance to high $\mathrm{pH}$, high temperature[44], compaction and biofouling [43]. In addition, Aquaporin-Incorporated Biomimetic Membranes are the high-performance FO 
membranes, which are made by incorporating Aquaporins into biomimetic membranes [45]. The poor mechanical stability and the complex fabrication process of this type of membranes are its main drawback [46]. In this respect, thin film composite (TFC) membranes seem to be more practical and superior to the old-style integrally skinned asymmetric membranes. They can function under a wider $\mathrm{pH}$ variation, resist high temperature media and are stable to high pressure and biodegradation problems [47]. A TFC membrane consists of at least two distinct layers, a top active layer and a porous substrate, each layer plays its special role [48]. In contrast to the integrally skinned asymmetric membranes, in which both active layer and the substrate layer simultaneously are made from the same material, in TFC membranes the active layer and the substrate layer can be optimized individually by using different materials and methods [49]. Thus, TFC membranes usually exhibit higher water flux and salt rejection relative to integrally skinned membranes in RO. Similarly, TFC membranes may exhibit better performances in FO than that of integrally skinned asymmetric membranes [50]. Despite these advantages, TFC membranes still suffer from the internal concentration polarization (ICP) in FO which severely diminishes the flux [39, 51-53].

Review articles on FO process have been published on topics such as basic principle of FO, challenges and application $[2,43,46,48,54]$; hybrid FO process $[21,37,55-57]$; draw solutes $[6$, 58, 59]; membrane fouling [60-63] and membrane material and fabrication [39, 64-68]. However, a review presenting detailed information on the fabrication and development of TFC FO membranes as the most widely used membrane in this area is currently lacking. This paper presents an overview on the recent advances in TFC FO membrane and the important factors such as the method and material of synthesizing active layer and substrate, which affect the TFC performance. The methods that are used for improving water flux $\left(\mathrm{J}_{\mathrm{v}}\right)$ and reducing the ratio of reverse salt flux $\left(\mathrm{J}_{\mathrm{s}}\right)$ (i.e. reverse solute diffusion from draw solution to feed solution) to water flux (specific reverse salt flux $\left.\left(\mathrm{J}_{\mathrm{S}} / \mathrm{J}_{\mathrm{v}}\right)\right)$ in TFC FO membrane are described. Also, in the conclusion section, a comprehensive roadmap based on the reviewed papers is proposed and discussions are made on the future direction for the FO membrane research and development. In the authors' view, this review can offer a useful guideline for the fabrication of more ideal TFC FO membranes and the promotion of FO process.

The classification of TFC FO membranes in this review is shown in Fig. 1. Three types of TFC membranes are presented: a) TFC with a polyamide active layer (TFC-A), b) Thin film nanocomposite (TFC-N) c) Double-skinned TFC (TFC-D). "TFC-A" includes TFC FO membranes, either modified or unmodified, with a polyamide active layer without nanomaterials, "TFC-N" includes all TFC FO membranes that contain nanomaterials and "TFC-D" includes TFC FO membranes with two active layers. 


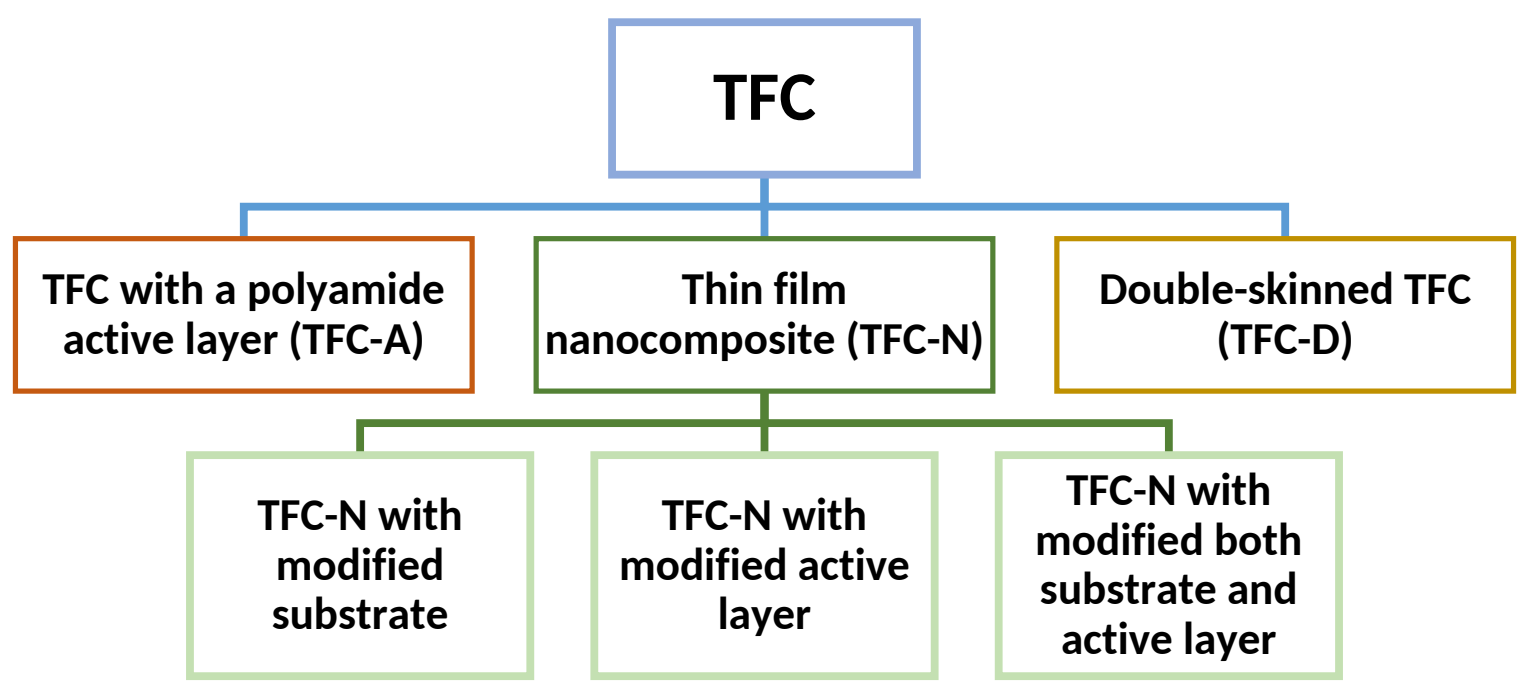

1

Fig 1: The classification of TFC membranes used in FO process

The TFC-A type is further divided into two groups according to their configuration: a) flat sheet and b) hollow fiber. To the best of the authors' knowledge, TFC-N membranes for FO application are mostly fabricated in the flat sheet configuration. Although the TFC-D type has been fabricated in the two different configurations, the number of TFC-D membranes are small. Hence, TFC-N and TFC-D membranes are discussed without splitting into two different configurations. It should be noted that many effects of membrane preparation on membrane performance are common for both flat sheet and hollow fiber configuration.

\section{Thin film composite membrane (TFC)}

Since 1980, TFC membranes have been utilized for water desalination by RO. In spite of the advances and improvements of membranes over the past years, the principles of their design have not been changed fundamentally. Polyamide TFC membranes are currently the most popular membranes for desalination in the commercial market [49].

\subsection{Synthesizing porous substrate}

As mentioned earlier, the TFC membrane consists of two distinct layers, the thin selective layer and the porous substrate. The latter layer is produced typically from polysulfone (PSF) and polyethersulfone (PES) by phase inversion method [39]. PSF and PES are the preferred material thanks to their good chemical and suitable mechanical resistance [66]. Occasionally, in the stage of preparing the membrane substrate, the polymer solution is cast on a polyester backing material [69]. Both woven and nonwoven polyester can be used [70]. The backing material does not pose 
any resistance against the flow of water and is only used to enhance the mechanical strength of the membrane [69]. A three-layer membrane is schematically shown in Fig. 2.

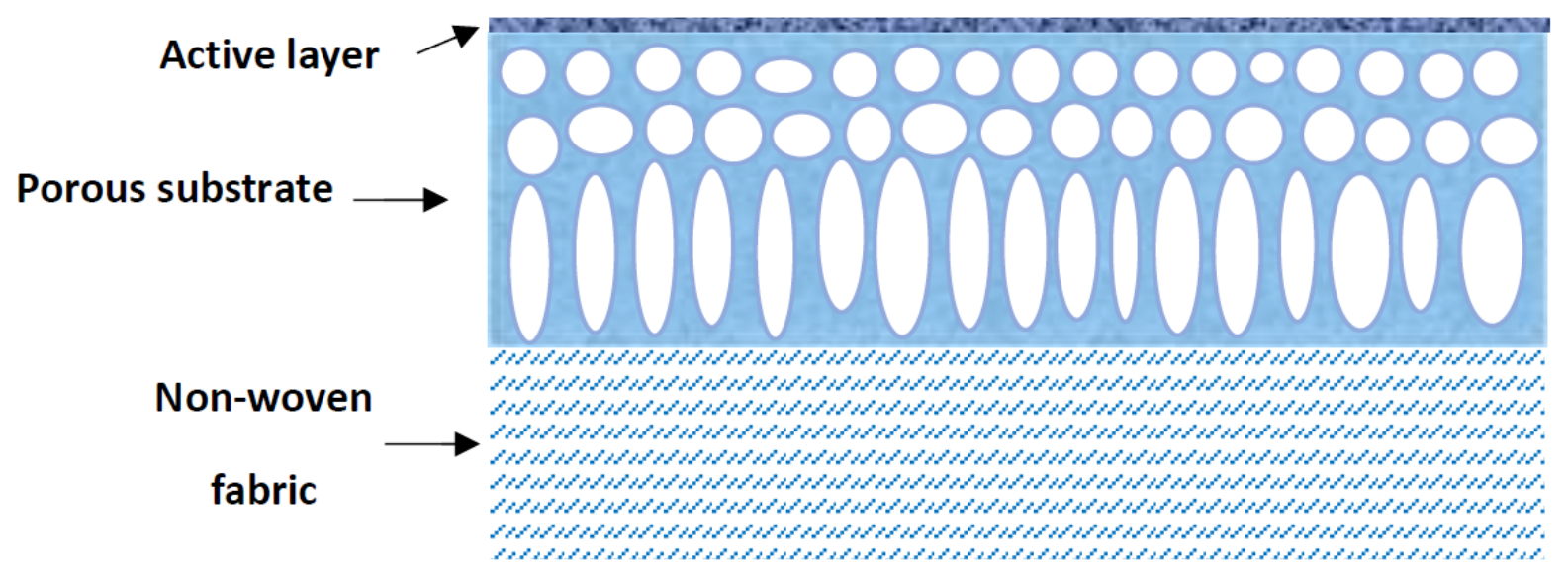

Fig 2: Diagram of a three-layer TFC membrane

Although the phase inversion method is one of the most prevalent methods for synthesizing the substrate of FO membranes, it has been encouraged recently to use the electro-spinning method to produce nanofiber polymeric substrate instead of the phase inversion method because it yields more satisfactory results in terms of the FO Flux [39, 71]. Electro-spinning applies a high electric field to make nanofibers from a dope solution [72]. In this process, both the curvature and tortuosity of the substrate are diminished while the porosity is enhanced $[39,71,73]$. Accordingly, the structural parameter $(S)$ of the produced membrane, defined as (thickness $\times$ tortuosity/porosity) [43] diminishes compared to the conventional membranes. This parameter is defined to evaluate the degree of internal concentration polarization. Nevertheless, the mechanical strength of the nanofiber layer is lower than that of the conventional substrates [39, 71]. Fig. 3 demonstrates the schematics of fabricating flat sheet substrate for FO process applying phase inversion and electro17 spinning methods. 


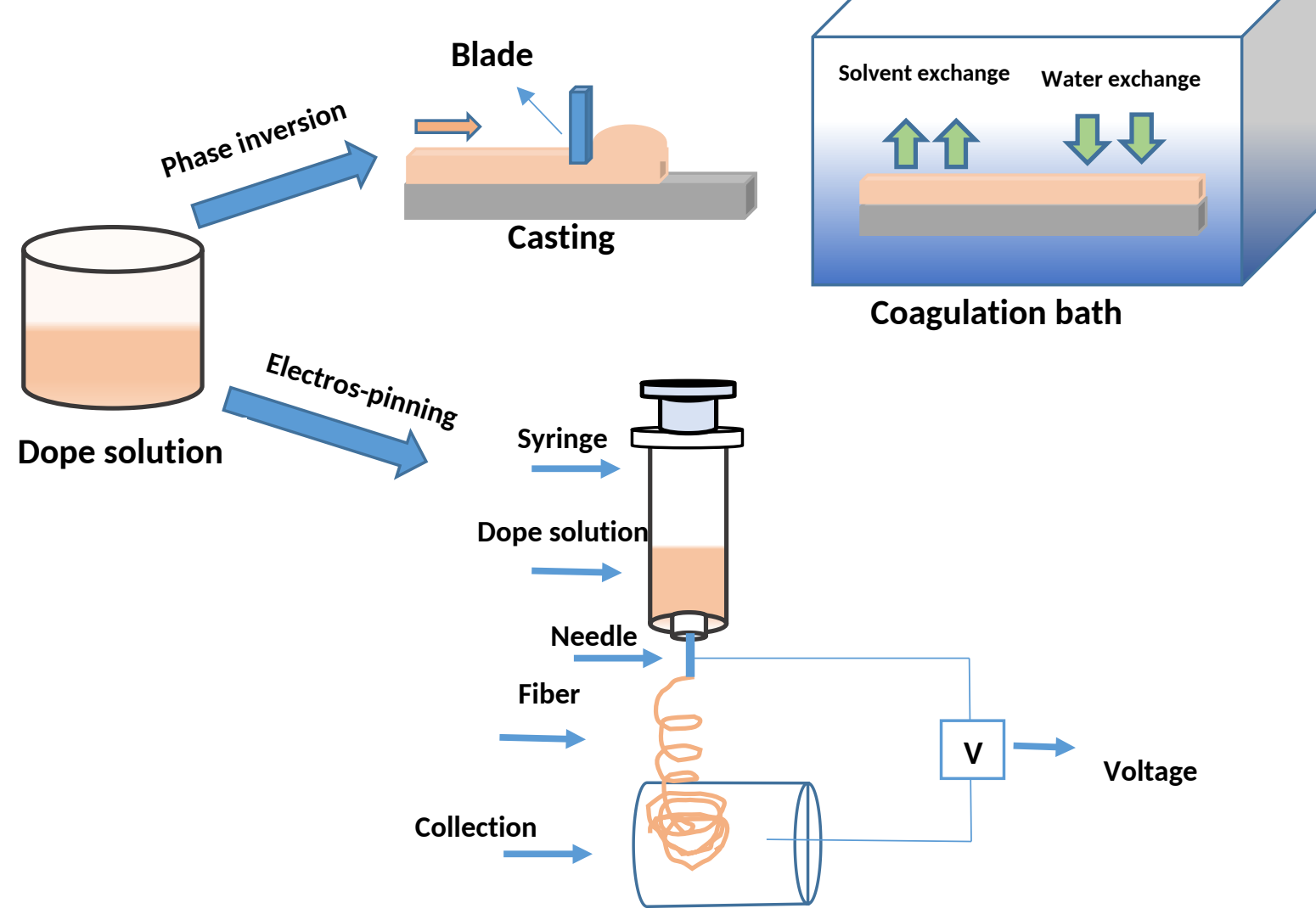

2 Fig 3: Flat sheet substrate fabricating techniques: (a) Phase inversion process (b) Electro-spinning process

\subsection{Synthesizing active layers}

5 Polyamide (PA) active layer is synthesized by the interfacial polymerization method using two 6 monomers, namely m-Phenylenediamine (MPD) in aqueous phase and Trimesoyl Chloride (TMC) 7 in organic phase [74, 75]. Fig. 4 demonstrates polyamide formation by the condensation reaction 8 of the two monomers, which takes place at the interface between the aqueous and organic phase.

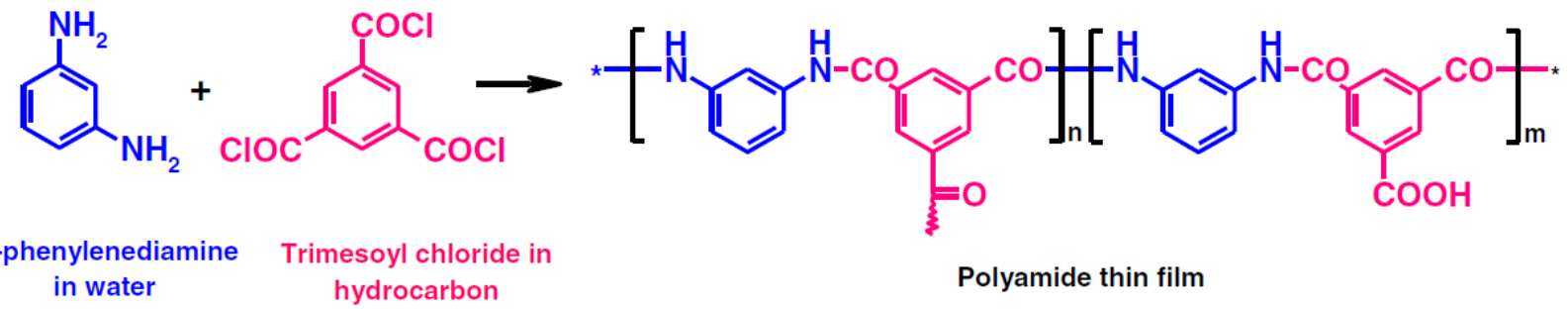

Fig 4: Interfacial polymerization reaction between MPD in water and TMC in n-hexane [74]

11 Other monomers can also be used to synthesize polyamide. Lau et al. [74] reviewed the common 12 monomers used in the synthesis of TFC membranes. They include amine monomer, such as 
Piperazine (PIP) and P-phenylene diamine (PPD) and Triethanolamine (TEOA) as well as Acyl chloride monomer, such as Isophthaloyl chloride(IPC), 5-isocyanato-isophthaloyl chloride (ICIC) and 5-chloroformyloxy-isophthaloylchloride (CFIC). Similarly, Li et al. [39] reviewed the monomers used in the synthesis of an active layer of TFC membranes and compared the properties of the synthesized active layer. In a wide range of monomers used in IP reaction to prepare the PA layer, MPD and TMC are the two most popular monomers [44]. Both MPD and TMC contain a benzene ring. Therefore, membranes fabricated by applying this chemistry are identified as fully aromatic PA membranes [76]. Indeed, aromatic cross-linked PA layer with high water flux and [77] high $\mathrm{NaCl}$ rejection[76] has mostly been provided by MPD and TMC monomers.

\subsection{Performance of TFC-A Flat sheet membranes}

To synthesize a high-performance TFC-A membrane, the properties of the substrate and the active layer of the membrane should be optimized [39]. The type of the polymer and its concentration, selecting a suitable solvent for preparing the casting solution, precipitation medium, and the use of additives are among the most effective factors in preparing the substrate [10,78-81] by phase inversion method. Various factors are involved in the final structure and performance of TFC membranes. The key parameters for the formation of the active layer by interfacial in-situ polymerization include the concentration of monomers, ratio of the monomers, type of solvent, time period of the interfacial polymerization reaction, and additives in the aqueous or organic solution [82-84].

\subsubsection{Effect of MPD and TMC concentrations on active layer}

Wei et al. [84] investigated the effect of the parameters affecting the active layer performance of a polyamide TFC-A membrane with PSF substrate. In particular, they examined the effect of MPD and TMC concentrations on the separation properties of the fabricated membranes. They found out that an increase in MPD concentration at a constant TMC concentration decreases the permeability and increases the salt rejection. It was most likely, due to the formation of denser active layer via higher degree of cross-linking. On the other hand, when TMC concentration is increased at a constant MPD concentration, the water permeability increases while salt rejection decreases, due to the increase in acyl chloride content and decrease in the extent of the crosslinking. They also stated that membranes with less salt rejection have a greater reverse solute diffusion and thus higher ICP. The performance of FO membrane is controlled by both permeability and salt rejection. When the main limiting factor of water flux is the friction loss, and also when the concentration of the draw solution is low, permeability has a greater effect than the salt rejection on water flux. Conversely, when the ICP is severe, salt rejection plays a more important role to govern the water flux. In the latter case, it is recommended to make the membrane active layer denser. 
Fig. 5 indicates the effect of MPD concentration on the water flux and specific reverse salt flux in two different orientations, FO (AL-FS) or PRO (AL-DS) orientation, and using two different draw solution concentrations. Specific reverse salt flux represents the selectivity which is unfavorable in different aspects: a) increase in the reverse solute diffusion causes aggravation of membrane fouling and increases accumulation of solutes in the feed solution side; $b$ ) it also increases the cost of replacing the draw solution. Water flux dependence on TMC concentration in a FO process is as complex as the effect of MPD concentration on the water flux. Furthermore, the optimization in FO is contingent upon the details of the applied conditions including the draw solution concentration and the membrane orientation. An optimal membrane in a particular condition does not necessarily yield the same results under new conditions. In the next sections the effect of the factors such as method and material of synthesizing substrates, that affect the TFC-A performance are discussed for polymers that are often used for substrate preparation.
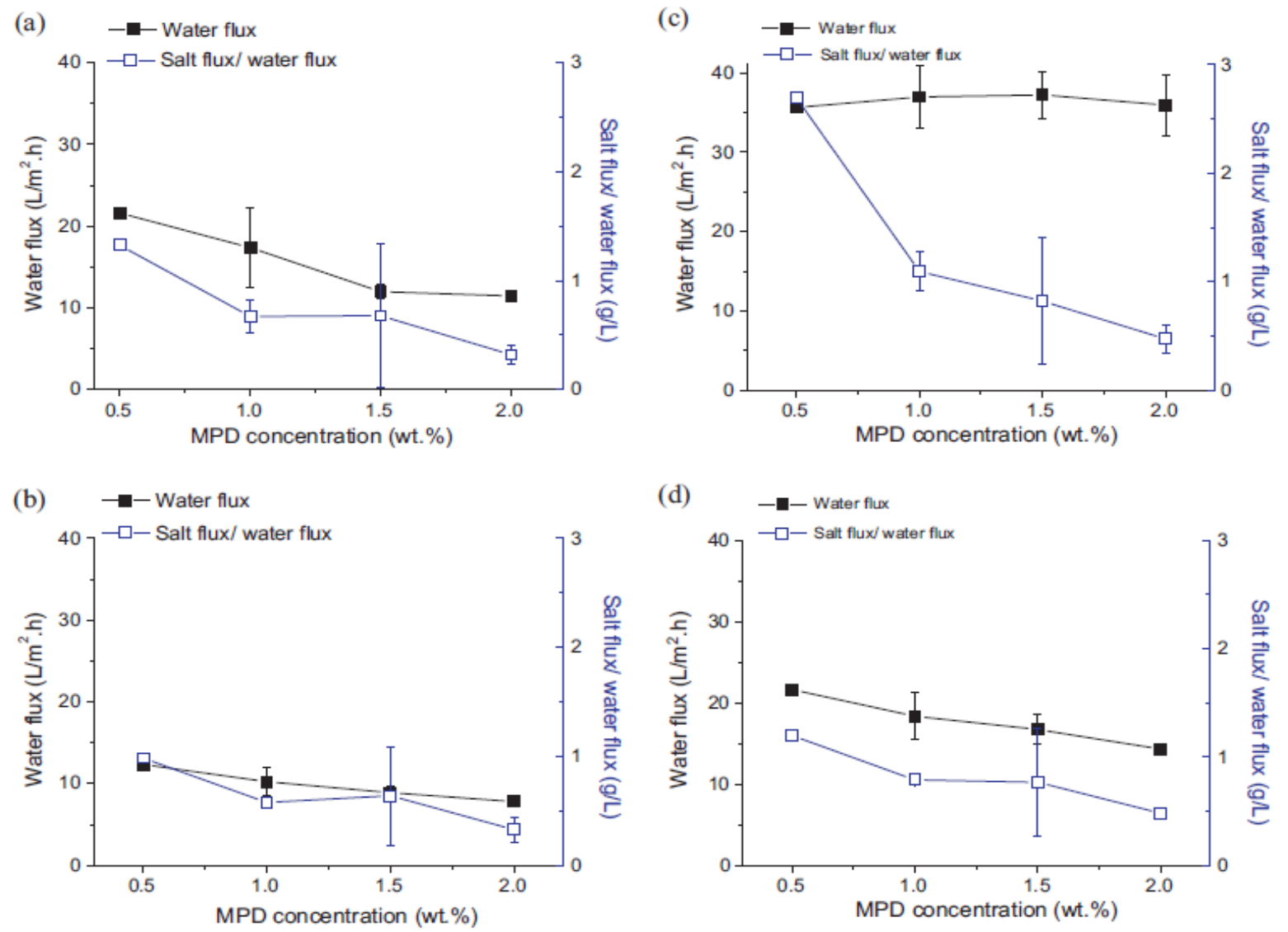

Fig 5: Performance of FO of TFC-A membranes fabricated with various MPD concentrations at fixed TMC concentration of $0.5 \mathrm{wt} . / \mathrm{v} \%$. (a) AL-DS and (b) AL-FS orientations for a $0.5 \mathrm{M} \mathrm{NaCl}$ draw solution; (c) AL-DS and (d) AL-FS orientations for a $2.0 \mathrm{M} \mathrm{NaCl}$ draw solution. The feed solutions contained $10 \mathrm{mM} \mathrm{NaCl}$ [84]. 


\subsubsection{PSF as substrate modified with polydopamine}

2 Han et al. [85] used a natural polymer called polydopamine (PDA) to improve the separation 3 performance of FO composite membranes. Polydopamine, as a hydrophilic polymer with adhesive

4 properties, has been used to enhance antifouling property in UF, NF, and RO by enhancing 5 hydrophilicity of the active layer and substrate of the membrane. The presence of a hydrophilic 6 substrate is also essential in FO membrane to mitigate the effect of ICP and to enhance the water 7 flux. In their work, PDA was coated on the top surface of a PSF substrate via self-polymerization 8 before the active layer was synthesized by in-situ polymerization. Experimental results have shown

9 the increase in water flux and salt rejection as well as the reduction of ICP. In addition to enhancing 10 the hydrophilicity of the internal walls of the substrate, PDA plays another positive role in 11 developing a polyamide active layer by creating a smooth hydrophilic surface with small pores. 12 Moreover, PDA interacts with TMC monomers and makes the active layer defect-free. It also 13 results in enhanced stability between the active layer and substrate.

\subsubsection{Polyacrylonitrile (PAN) as substrate}

Klaysom et al. [83] synthesized a TFC membrane with polyacrylonitrile (PAN) substrate. PAN is more hydrophilic than PSF and PES. In addition, PAN has high thermal and chemical resistance as well as resistance against the normal solvents but the nitrile group is sensitive to alkaline environment [66]. The main aim of Klaysom et al. work was to determine the essential parameters 20 that affect the fabrication of TFC-A membranes especially in the interfacial polymerization stage. 21 The parameters considered were the type of the additives and the solvent evaporation period. As 22 shown in Fig. 6, the substrate consists of three parts, namely the dense skin layer, the upper part 23 of sublayer with smaller macrovoids and the lower part of the substrate with larger macrovoids. 24 They have reported that the structural parameter of the PAN substrate was lower than that of the 25 commercial HTI membranes [83]. 
a

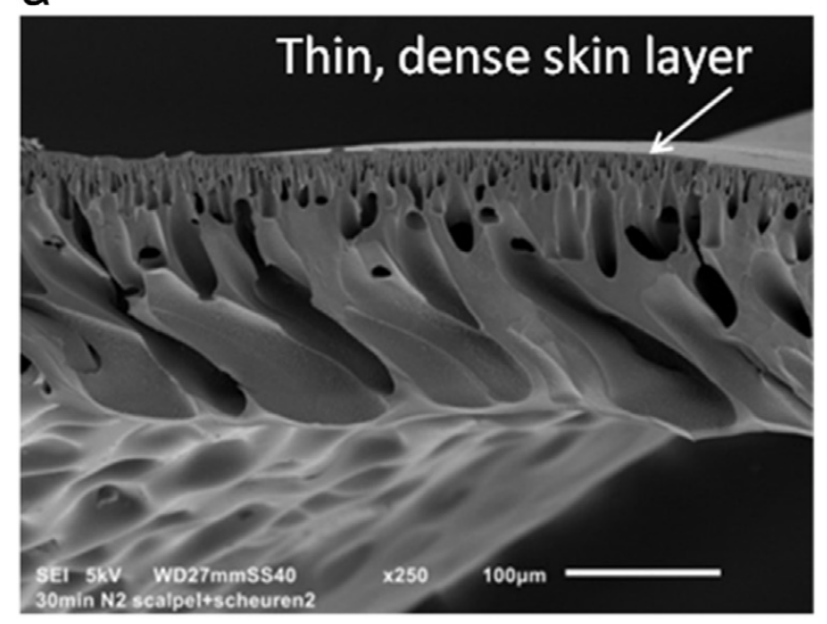

\section{C}

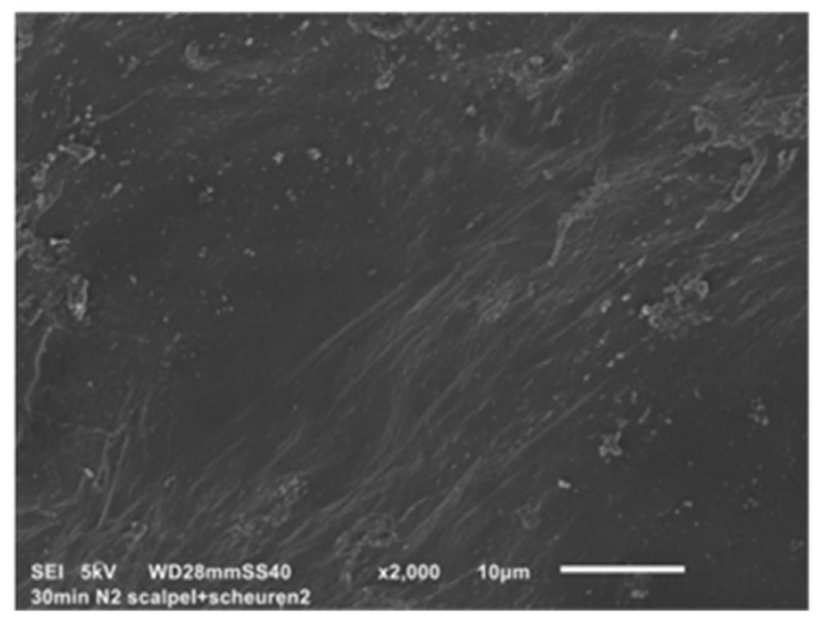

b

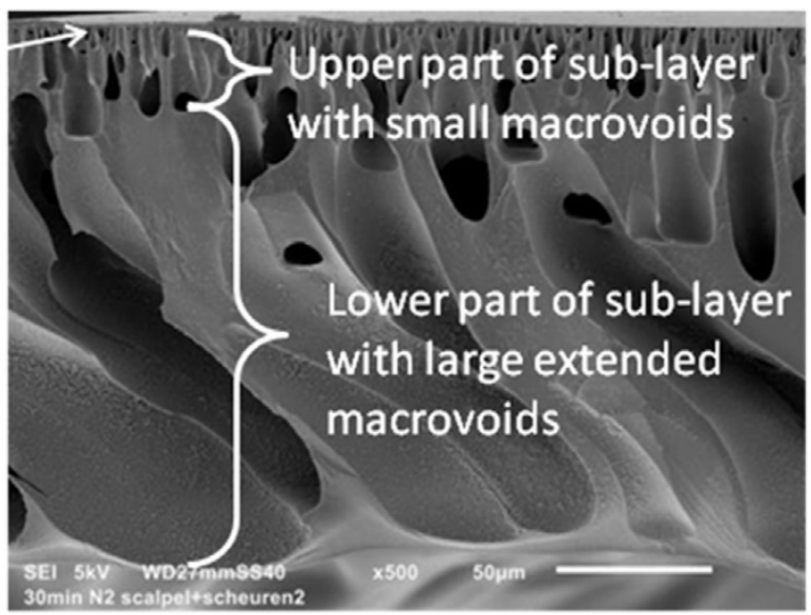

d

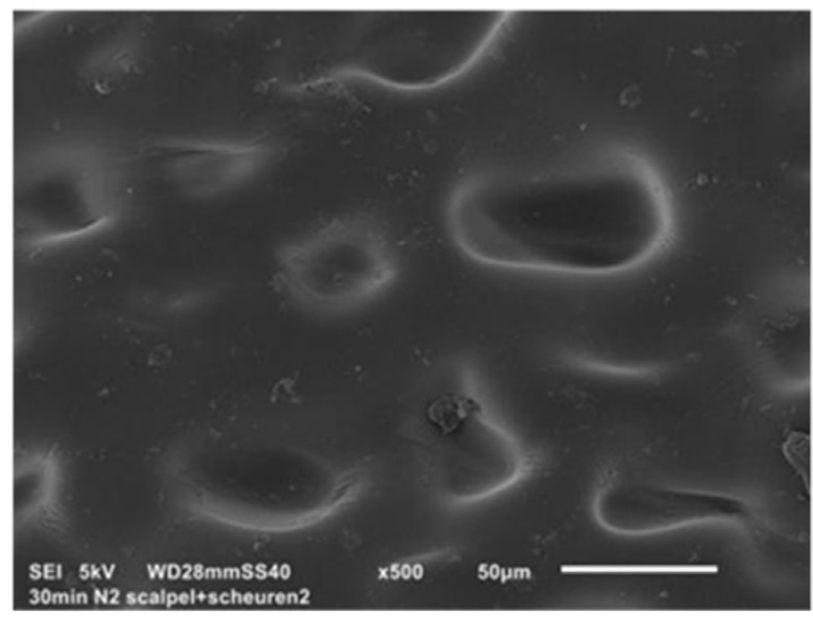

Fig 6: SEM images of (a and b) cross-section, (c) top surface, and (d) bottom surface of the PAN support layer [83]

After fabrication of the substrate, the active layer was synthesized via interfacial polymerization technique utilizing MPD and TMC as monomers. First, the substrate was immersed in an MPD aqueous solution for $30 \mathrm{~min}$. After clearing the extra amine solution from the substrate surface by a clean cloth, the substrate surface was dried for 1-3 min in a fume hood at ambient temperature. Then, the substrate surface was contacted with the TMC organic solution for 20-80 s, followed by washing with n-hexane to remove the unreacted solution. The membranes were further dried at the ambient condition for $1 \mathrm{~min}$ before being stored in deionized water at room temperature prior to use. By increasing the drying time after soaking in the amine solution, the salt rejection increased while the permeability gradually diminished. In addition, the surface roughness of the membrane displayed a decreasing trend [83]. It is well known that the polycondensation reaction takes place in the organic phase. Therefore, the stability of the polyamide layer depends on the closeness of the two phases interface to the surface of the substrate. If the substrate surface is cleared and cleaned well, MPD solution lies inside the substrate and exactly in the vicinity of the surface. This 
in turn causes the formation of an absolutely stable and strong active layer. On the other hand, if the substrate surface is not sufficiently cleaned and dried to make it free from the amine solution, the contact area of the two phases might be detached from the top substrate surface causing the formation of a flimsy and brittle polyamide layer at a distance off the substrate surface [86]. The reaction time also affects the surface morphology and permeability. By lengthening the reaction time, due to the increase in the thickness of the formed active layer, the permeability decreases. The effect of reaction time on permeability is more pronounced between 20 and $40 \mathrm{~s}$ [83], since as the time lengthens, the active layer formation rate decreases while the active layer thickness remains constant [87]. Therefore, the effect of reaction time, especially after $40 \mathrm{~s}$ on the surface morphology, is higher than its effect on the permeability [83]. Khorshidi et al. [88] also studied the effect of reaction time in a range of 15 to $60 \mathrm{~s}$, and observed that the increase in reaction time slightly decreased the permeability. Fig. 7 shows the formation of the active layer on the PAN substrate as a function of drying and reaction time. As for the effect of the substrate pore size, when the pore is small, MPD transfer occurs by diffusion and simple convection whereby a crosslinked thin film with a nodular structure is developed. When the pore size is large, MPD transfers to the organic phase much faster due to the Marangoni effect caused by the surface tension gradient between the two phases. This results in the rapid migration of MPD monomers. This rapid migration tends to push the initially formed nascent outward and twist the formed film, thus ridge and valley structure is produced [83].
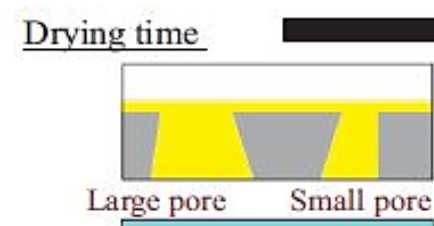

Small pore
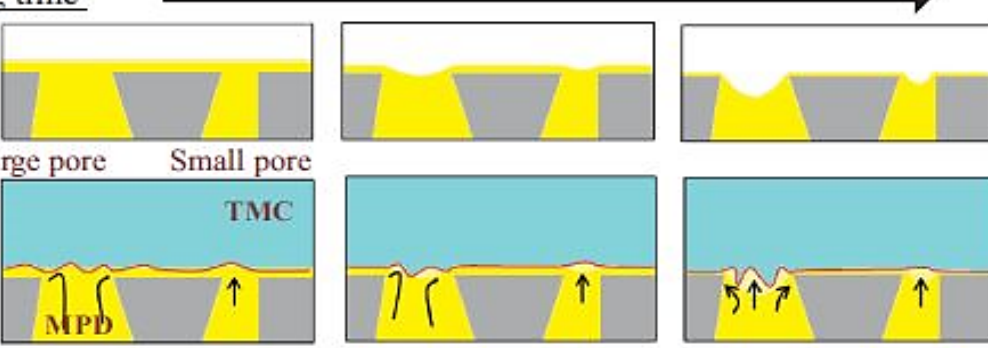

Smoother surface Reaction time
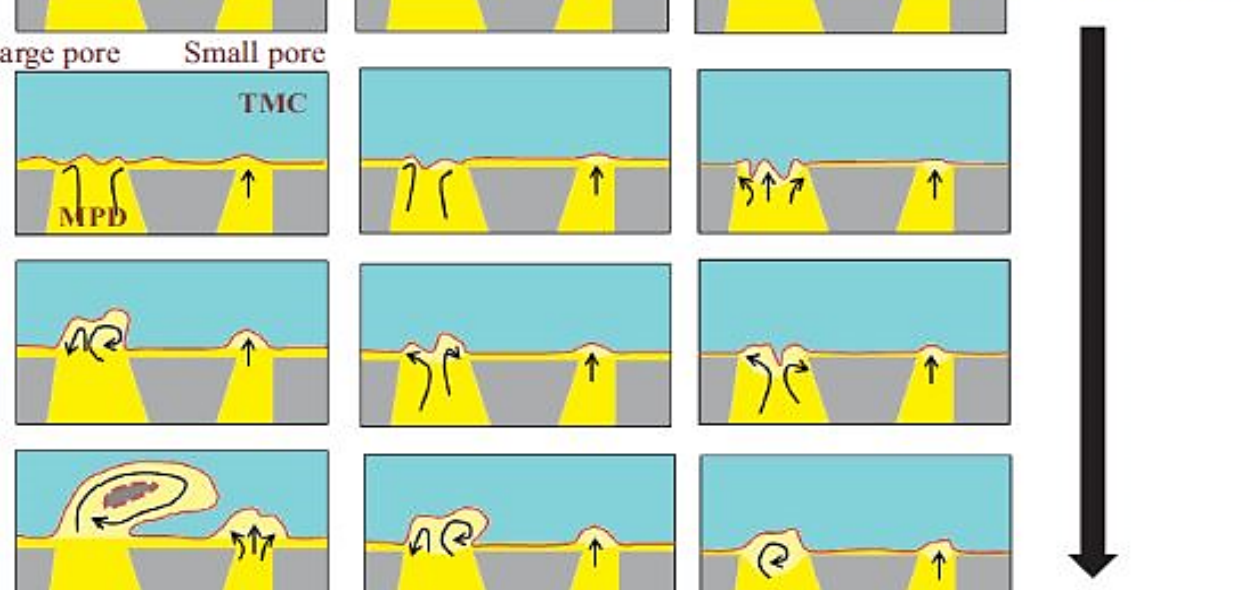

Rougher surface
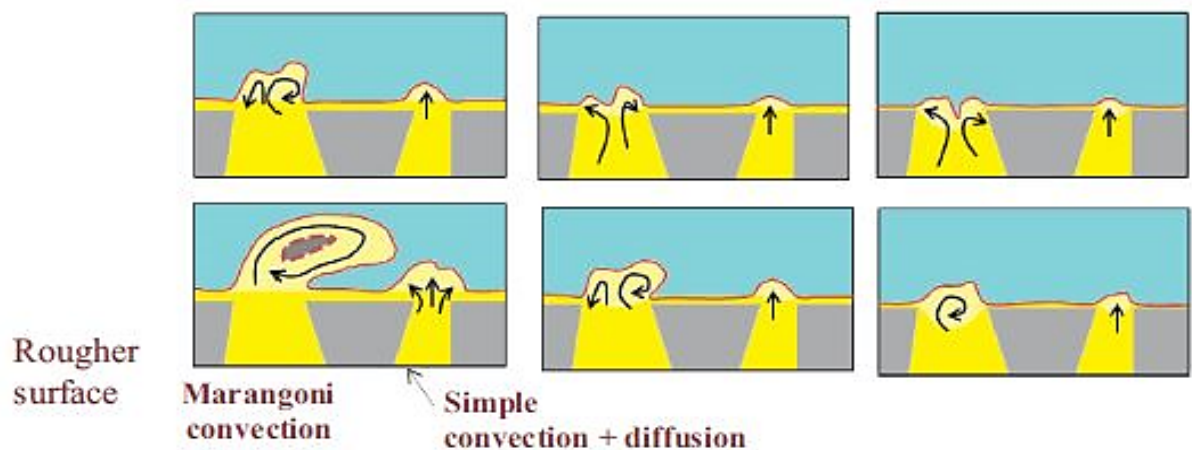

convection + diffusion

Fig 7: Scheme showing polyamide thin film formation mechanism [83] 


\subsubsection{Nylon 6.6 as substrate}

Huang et al. [89] also investigated the effect of the substrate pore size on the properties of the active layer including morphology, extent of crosslinking, mechanical integrity, selectivity, and permeability. In their research, four types of microfiltration substrates made of Nylon 6.6 with different pore sizes were provided by $3 \mathrm{M}$ Purification Inc. Nylon 6,6 is a conventional polymer for plastic and textile industry. It has a semi-crystalline structure, i.e. posing good thermal, mechanical and chemical properties. Additionally, it is more hydrophilic compared to the common polymers such as PSF. However it has less swelling propensity than other conventional hydrophilic polymers, such as cellulose acetate (CA) [90]. The general structures, as well as physiochemical properties of the substrates, were identical. In a range of pore sizes 0.025 to $0.45 \mu \mathrm{m}$, they concluded that the substrate pore size had no effect on the thickness of the active layer. However, with an increase in the pore size, the degree of crosslinking diminished, leading to the decrease in salt rejection. Additionally, they found out that the membrane mechanical strength was higher for small pores. Considering all these factors, they concluded that the substrate pore size of $0.2 \mu \mathrm{m}$ resulted in the optimum TFC membrane. Although membranes with fewer pores have a greater extent of crosslinking and higher selectivity, the balance between selectivity and permeability is important to achieve better performance. Membranes with smaller pores show lower permeabilities but higher structural parameters. Further, membranes with the pore size of $0.45 \mu \mathrm{m}$ had a lower water flux compared to the other membranes, which was due to an increase in reverse salt flux, hence increase in ICP, and reduction of driving force. An important conclusion that can be drawn, is that to synthesize high-performance membranes, the thickness, tortuosity and porosity should be controlled to minimize the structural parameter. Also, the pore size of the substrate and its effect on the structural parameter along with the performance of the membrane active layer should be taken into account [89]. Contrary to Huang et al. [89], Singh et al. [91] reported that the pore size of the PSF substrate has an effect on the active layer thickness. It is probably due to the higher hydrophobicity of PSF than Nylon 6,6, which caused the higher resistance for MPD diffusion in the smaller pores.

\subsubsection{Polyethylene as substrate modified with polydopamine}

Kown et al. [92] fabricated a high performance and outstanding durable membrane with polydopamine-modified polyethylene (DPE) substrate having an active layer formed by a unconventional interfacial polymerization method. They used an aromatic solvent-based i.e. toluene instead of an aliphatic organic solvent i.e. n-hexane, with the same monomer (TMC) in their method. The polydopamine coating uniformly hydrophilized the hydrophobic polyethylene (PE) surface, yielding a long-term stable operation. The highly porous and thin substrate structure was protected after modification of the substrate with PDA. In addition, the use of the toluenebased IP process allowed for the formation of a highly selective polyamide active layer on the hydrophilic DPE substrate, which was superior to the conventional IP method. Indeed, toluene as an aromatic hydrocarbon solvent has lower interfacial tension with water and greater MPD 
1 solubility than $\mathrm{n}$-hexane as an aliphatic hydrocarbon, which can accelerate MPD diffusion and

2 promote the IP reaction. Thus, the prepared TFC membrane with this method presented much 3 higher FO performance. Table 1 summarizes the latest studies published in the literature. The table 4 includes water flux and specific reverse salt flux of flat sheet TFC membranes with a polyamide 5 active layer. Experimental results in the two different modes (AL-FS(FO)) and AL-DS(PRO) are 6 separately reported. 
Table 1: Summary of the studies on flat sheet TFC-A FO membranes

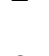

3

4

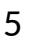

6

PES

sulphonated copolymer polyethersulfone and polyphenylsulfone support Performance

\begin{tabular}{lll}
\hline support & Performance & Experimental condition
\end{tabular}

(PES-co-sPPSU 11)

\begin{tabular}{cccc}
\multicolumn{2}{c}{ AL-FS(FO) } & \multicolumn{2}{c}{ AL-DS(PRO) } \\
\hline $\mathrm{J}_{\mathrm{v}}$ & $\mathrm{J}_{\mathrm{s}} / \mathrm{J}_{\mathrm{v}}$ & $\mathrm{J}_{\mathrm{v}}$ & $\mathrm{J}_{\mathrm{s}} / \mathrm{J}_{\mathrm{v}}$ \\
$(\mathrm{LMH})$ & $(\mathrm{g} / \mathrm{L})$ & $(\mathrm{LMH})$ & $(\mathrm{g} / \mathrm{L})$
\end{tabular}

$\begin{array}{cr}\text { Feed } & \begin{array}{r}\text { Draw } \\ \text { solution }\end{array} \\ \text { solution }\end{array}$

cross-flow velocity

Intrinsic

properties

ref

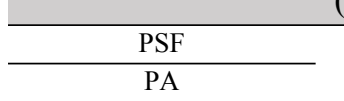

12

0.408

$20.5 \quad 0.287$

$0.5 \quad \mathrm{~m}$

$500 \mathrm{ml} / \mathrm{min}$

$\mathrm{A}=1.78 \mathrm{LMH} / \mathrm{bar}$

$\mathrm{B}=9.4 \times 10^{-8} \mathrm{~m} / \mathrm{s}$

$\mathrm{S}=0.67 \mathrm{~mm}$

$\mathrm{R}=93.4$

$=0.73 \mathrm{LMH} / \mathrm{bar}$

$\mathrm{B}=0.25 \mathrm{LMH}$

$\mathrm{S}=3.24 \times 10^{-4} \mathrm{~m}$

9

$$
\mathrm{R}=91
$$

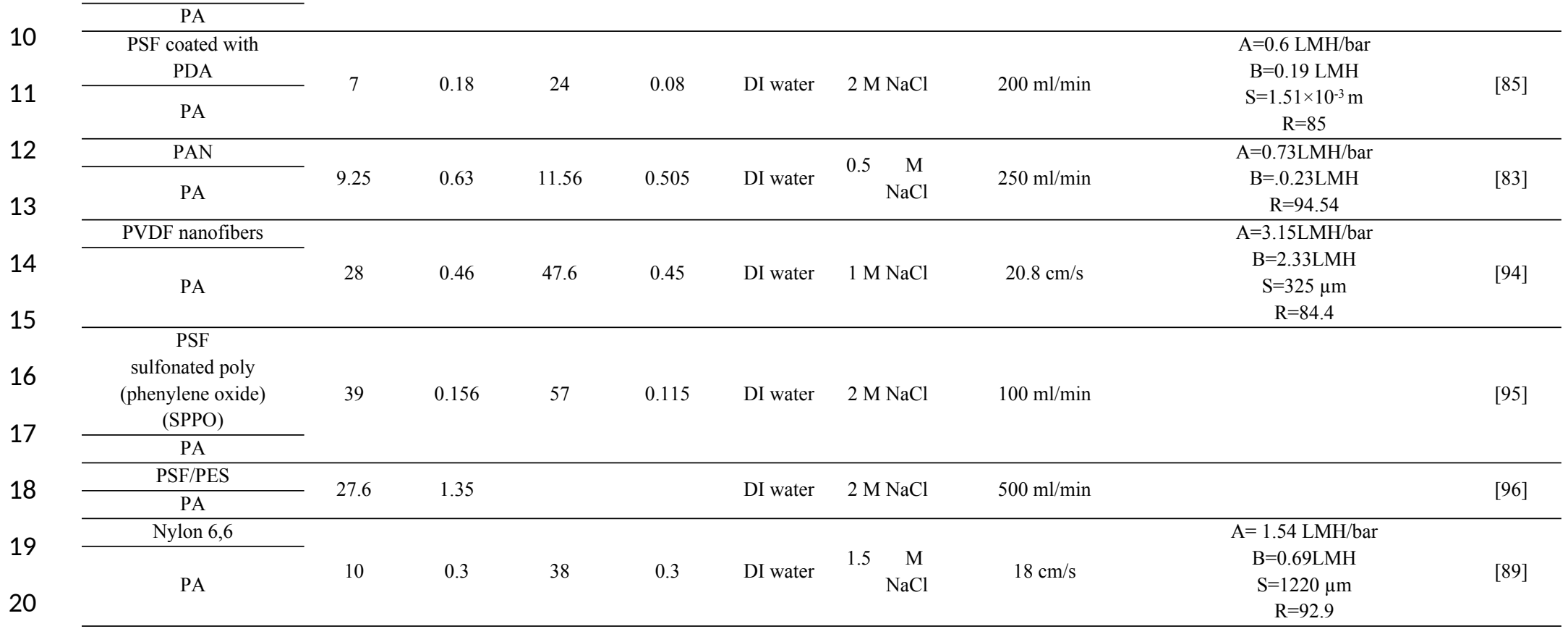

21 
Table1:(continued)

\begin{tabular}{|c|c|c|c|c|c|c|c|c|c|}
\hline \multirow{3}{*}{$\begin{array}{c}\text { support } \\
\text { Active layer }\end{array}$} & \multicolumn{4}{|c|}{ Performance } & \multicolumn{3}{|c|}{ Experimental condition } & \multirow[b]{3}{*}{$\begin{array}{c}\text { Intrinsic } \\
\text { properties }\end{array}$} & \multirow[b]{3}{*}{ ref } \\
\hline & \multicolumn{2}{|c|}{ AL-FS(FO) } & \multicolumn{2}{|c|}{ AL-DS(PRO) } & \multirow[b]{2}{*}{$\begin{array}{c}\text { Feed } \\
\text { solution }\end{array}$} & \multirow[b]{2}{*}{$\begin{array}{r}\text { Draw } \\
\text { solution }\end{array}$} & \multirow[b]{2}{*}{ cross-flow velocity } & & \\
\hline & $\begin{array}{r}\mathrm{J}_{\mathrm{v}} \\
(\mathrm{LMH}) \\
\end{array}$ & $\begin{array}{l}\mathrm{J}_{\mathrm{s}} / \mathrm{J}_{\mathrm{V}} \\
(\mathrm{g} / \mathrm{L})\end{array}$ & $\begin{array}{r}\mathrm{J}_{\mathrm{V}} \\
(\mathrm{LMH})\end{array}$ & $\begin{array}{l}\mathrm{J}_{\mathrm{s}} / \mathrm{J}_{\mathrm{v}} \\
(\mathrm{g} / \mathrm{L})\end{array}$ & & & & & \\
\hline PAN & & & & & & & & $\mathrm{A}=1.13 \mathrm{LMH} / \mathrm{bar}$ & \multirow[b]{2}{*}{ [97] } \\
\hline $\mathrm{PA}$ & 31.3 & 0.163 & & & DI water & $1 \mathrm{M} \mathrm{NaCl}$ & $750 \mathrm{ml} / \mathrm{min}$ & $\begin{array}{c}\mathrm{B}=0.335 \mathrm{LMH} \\
\mathrm{S}=112 \mu \mathrm{m} \\
\mathrm{R}=91.4\end{array}$ & \\
\hline PVDF nanofibers/ nylon 6,6 & \multirow[b]{2}{*}{30} & \multirow[b]{2}{*}{0.3} & \multirow[b]{2}{*}{41} & \multirow[b]{2}{*}{0.47} & \multirow[b]{2}{*}{ DI water } & \multirow{2}{*}{$\begin{array}{rr}1.5 & \mathrm{M} \\
\mathrm{NaCl}\end{array}$} & \multirow[b]{2}{*}{$18 \mathrm{~cm} / \mathrm{s}$} & $\mathrm{A}=1.28 \mathrm{LMH} / \mathrm{bar}$ & \multirow[b]{2}{*}{ [98] } \\
\hline (a) & & & & & & & & $\begin{array}{c}\mathrm{B}=0.25 \mathrm{LMH} \\
\mathrm{S}=193 \mu \mathrm{m}\end{array}$ & \\
\hline $\begin{array}{c}\begin{array}{c}\text { PSF/sulfonated } \\
\text { polysulfone (sPSF) }\end{array} \\
\text { PA }\end{array}$ & 29.02 & 0.18 & 49.92 & 0.18 & DI water & $1 \mathrm{M} \mathrm{NaCl}$ & $6.4 \mathrm{~cm} / \mathrm{s}$ & $\begin{array}{c}\mathrm{A}=1.93 \mathrm{LMH} / \mathrm{bar} \\
\mathrm{B}=0.31 \mathrm{LMH} \\
\mathrm{S}=220 \mu \mathrm{m}\end{array}$ & [99] \\
\hline $\begin{array}{c}\text { PES } \\
\text { SPES } \\
\text { PA }\end{array}$ & 35.1 & 0.28 & 42.1 & 0.26 & DI water & $2 \mathrm{M} \mathrm{NaCl}$ & & $\begin{array}{c}\mathrm{A}=2.9 \mathrm{LMH} / \mathrm{bar} \\
\mathrm{B}=5.1 \times 10^{-8} \mathrm{~m} / \mathrm{s} \\
\mathrm{S}=245 \mu \mathrm{m} \\
\mathrm{R}=91.1\end{array}$ & {$[100]$} \\
\hline $\begin{array}{l}\begin{array}{l}\text { PVDF nanofibers coated with } \\
\text { PVA (Polyvinyl alcohol) }\end{array} \\
\text { PA }\end{array}$ & 24.8 & 0.13 & 32.5 & 0.13 & DI water & $\begin{array}{r}0.5 \mathrm{M} \\
\mathrm{NaCl}\end{array}$ & $\begin{array}{l}13.88 \mathrm{~cm} / \mathrm{s} \\
500 \mathrm{ml} / \mathrm{min}\end{array}$ & $\begin{array}{c}\mathrm{A}=0.62 \mathrm{LMH} / \mathrm{bar} \\
\mathrm{B}=0.31 \mathrm{LMH} \\
\mathrm{S}=154 \mu \mathrm{m}\end{array}$ & {$[101]$} \\
\hline $\begin{array}{c}\text { PAN/PSF nanofibers } \\
\text { PA }\end{array}$ & & & 38.3 & 0.27 & DI water & $1 \mathrm{M} \mathrm{NaCl}$ & & $\begin{array}{c}\mathrm{A}=3.68 \mathrm{LMH} / \mathrm{bar} \\
\mathrm{B}=0.32 . \mathrm{LMH} \\
\mathrm{S}=34 \mu \mathrm{m}\end{array}$ & {$[102]$} \\
\hline CTA & & & & & & & & $\mathrm{A}=1.52 \mathrm{LMH} / \mathrm{bar}$ & \\
\hline PA & 11.79 & 0.58 & 14.58 & 0.69 & DI water & $\begin{array}{r}0.5 \mathrm{M} \\
\mathrm{NaCl}\end{array}$ & $333.3 \mathrm{ml} / \mathrm{min}$ & $\begin{array}{c}\mathrm{B}=0.27 \mathrm{LMH} \\
\mathrm{S}=516 \mu \mathrm{m} \\
\mathrm{R}=96\end{array}$ & {$[103$} \\
\hline $\begin{array}{c}\text { PES } \\
\text { PA } \\
\text { 2,5-disulfoaniline disodium } \\
\text { salt (DSA-2Na) }\end{array}$ & 12.6 & 0.126 & 16 & 0.11 & DI water & $\begin{array}{rr}0.5 & \mathrm{M} \\
\mathrm{NaCl}\end{array}$ & $1.3 \mathrm{~cm} / \mathrm{s}$ & $\begin{array}{c}\mathrm{A}=0.71 \mathrm{LMH} / \mathrm{bar} \\
\mathrm{B}=0.094 \mathrm{LMH} \\
\mathrm{R}=93.79\end{array}$ & {$[104]$} \\
\hline
\end{tabular}


Table1:(continued)

\begin{tabular}{|c|c|c|c|c|c|c|c|c|c|}
\hline support & \multicolumn{4}{|c|}{ Performance } & \multicolumn{3}{|c|}{ Experimental condition } & \multirow[b]{3}{*}{$\begin{array}{c}\text { Intrinsic } \\
\text { properties }\end{array}$} & \multirow{3}{*}{ ref } \\
\hline & \multicolumn{2}{|c|}{ AL-FS(FO) } & \multicolumn{2}{|c|}{ AL-DS(PRO) } & \multirow[b]{2}{*}{$\begin{array}{c}\text { Feed } \\
\text { solution }\end{array}$} & \multirow[b]{2}{*}{$\begin{array}{r}\text { Draw } \\
\text { solution }\end{array}$} & \multirow{2}{*}{ cross-flow velocity } & & \\
\hline Active layer & $\begin{array}{r}\mathrm{J}_{\mathrm{v}} \\
(\mathrm{LMH})\end{array}$ & $\begin{array}{l}\mathrm{J}_{\mathrm{s}} / \mathrm{J}_{\mathrm{v}} \\
(\mathrm{g} / \mathrm{L})\end{array}$ & $\begin{array}{r}\mathrm{J}_{\mathrm{v}} \\
(\mathrm{LMH})\end{array}$ & $\begin{array}{l}\mathrm{J}_{\mathrm{S}} / \mathrm{J}_{\mathrm{v}} \\
(\mathrm{g} / \mathrm{L})\end{array}$ & & & & & \\
\hline CA/PVDF nanofibers & \multirow[b]{2}{*}{31.3} & \multirow[b]{2}{*}{0.03} & & & \multirow[b]{2}{*}{ DI water } & \multirow{2}{*}{$\begin{array}{cc}0.5 & \mathrm{M} \\
& \mathrm{NaCl}\end{array}$} & \multirow{2}{*}{$\begin{array}{l}400 \mathrm{ml} / \mathrm{min} \\
13.88 \mathrm{~cm} / \mathrm{s}\end{array}$} & $\mathrm{A}=2.79 \mathrm{LMH} / \mathrm{bar}$ & \multirow[b]{2}{*}[105]{} \\
\hline PA & & & & & & & & $\begin{array}{c}\mathrm{B}=0.07 \mathrm{LMH} \\
\mathrm{S}=190 \mu \mathrm{m}\end{array}$ & \\
\hline $\begin{array}{l}\text { Poly (vinyl butyral) } \\
\text { (PVB) / poly (vinyl } \\
\text { chloride) (PVC) } \\
\text { PA }\end{array}$ & 29.37 & 0.11 & 49.85 & 0.1 & DI water & $1 \mathrm{M} \mathrm{NaCl}$ & $13.33 \mathrm{~cm} / \mathrm{s}$ & $\begin{array}{c}\mathrm{A}=1.75 \mathrm{LMH} / \mathrm{bar} \\
\mathrm{B}=8.99 \times 10^{-8} \mathrm{~m} / \mathrm{s} \\
\mathrm{S}=186 \mu \mathrm{m} \\
\mathrm{R}=94.98\end{array}$ & {$[106]$} \\
\hline PSF & \multirow[b]{2}{*}{25} & \multirow[b]{2}{*}{0.18} & \multirow[b]{2}{*}{54.5} & \multirow[b]{2}{*}{0.22} & \multirow[b]{2}{*}{ DI water } & \multirow[b]{2}{*}{$2 \mathrm{M} \mathrm{NaCl}$} & \multirow[b]{2}{*}{$1000 \mathrm{ml} / \mathrm{min}$} & $\mathrm{A}=1.51 \mathrm{LMH} / \mathrm{bar}$ & \multirow[b]{2}{*}{ [107] } \\
\hline $\begin{array}{c}\text { PA } \\
\text { polyethylenimine } \\
(\text { PEI })\end{array}$ & & & & & & & & $\begin{array}{c}\mathrm{B}=0.31 \mathrm{LMH} \\
\mathrm{S}=334.1 \mu \mathrm{m} \\
\mathrm{R}=85.1\end{array}$ & \\
\hline PAN & \multirow[b]{2}{*}{34.2} & \multirow[b]{2}{*}{0.17} & \multirow[b]{2}{*}{44.5} & \multirow[b]{2}{*}{0.19} & \multirow[b]{2}{*}{ DI water } & \multirow[b]{2}{*}{$1 \mathrm{M} \mathrm{NaCl}$} & \multirow[b]{2}{*}{$600 \mathrm{ml} / \mathrm{min}$} & $\mathrm{A}=3.26 \mathrm{LMH} / \mathrm{bar}$ & \multirow[b]{2}{*}[108]{} \\
\hline $\begin{array}{c}\text { PA } \\
\mathrm{PA}^{*} \text { (toluene as an } \\
\text { organic solvent) } \\
\end{array}$ & & & & & & & & $\begin{array}{c}\mathrm{B}=0.25 \mathrm{LMH} \\
\mathrm{S}=378 \mu \mathrm{m} \\
\mathrm{R}=98.1\end{array}$ & \\
\hline $\begin{array}{c}- \\
\text { PA }\end{array}$ & 80.54 & 0.44 & & & DI water & $1 \mathrm{M} \mathrm{NaCl}$ & & & [109] \\
\hline $\begin{array}{c}\text { Cyanoethyl Cellulose } \\
\text { (CEC) }\end{array}$ & 9.10 & 0.15 & 20.67 & 0.11 & DI water & $1 \mathrm{M} \mathrm{NaCl}$ & $480 \mathrm{~mL} / \mathrm{min}$ & $\begin{array}{c}\mathrm{A}=1.17 \mathrm{LMH} / \mathrm{bar} \\
\mathrm{B}=0.2 \mathrm{LMH} \\
\mathrm{S}=922 \mu \mathrm{m} \\
\end{array}$ & [110] \\
\hline $\begin{array}{c}\text { PES/ } \\
\text { sulfonated } \\
\text { polyetheretherketone } \\
\text { (sPEEK) }\end{array}$ & 11 & 1.06 & 23 & 1.5 & DI water & $\begin{array}{r}0.5 \mathrm{M} \\
\mathrm{NaCl}\end{array}$ & $250 \mathrm{ml} / \mathrm{min}$ & $\begin{array}{c}\mathrm{A}=1.9 \mathrm{LMH} / \mathrm{bar} \\
\mathrm{S}=383 \mu \mathrm{m} \\
\mathrm{R}=75\end{array}$ & [111] \\
\hline PES & & & & & & & & $\mathrm{A}=2.64 \mathrm{LMH} / \mathrm{bar}$ & \\
\hline PA & 21.6 & 0.77 & 29.3 & 0.66 & DI water & $1 \mathrm{M} \mathrm{NaCl}$ & $8 \mathrm{~cm} / \mathrm{s}$ & $\begin{array}{c}\mathrm{B}=2.35 \mathrm{LMH} \\
\mathrm{R}=82.53\end{array}$ & [112] \\
\hline DPE & & & & & & & & $\mathrm{A}=6.7 \mathrm{LMH} / \mathrm{bar}$ & \\
\hline $\begin{array}{l}\mathrm{PA}^{*} \text { (toluene as an } \\
\text { organic solvent) }\end{array}$ & 53 & 0.28 & 64.8 & 0.28 & DI water & $1 \mathrm{M} \mathrm{NaCl}$ & $600 \mathrm{ml} / \mathrm{min}$ & $\begin{array}{c}\mathrm{B}=0.68 \mathrm{LMH} \\
\mathrm{S}=168 \mu \mathrm{m} \\
\mathrm{R}=98.1\end{array}$ & [92] \\
\hline
\end{tabular}


Table1:(continued)

\begin{tabular}{|c|c|c|c|c|c|c|c|c|c|}
\hline \multirow{3}{*}{$\begin{array}{c}\text { support } \\
\text { Active layer }\end{array}$} & \multicolumn{4}{|c|}{ Performance } & \multicolumn{3}{|c|}{ Experimental condition } & \multirow[b]{3}{*}{$\begin{array}{c}\text { Intrinsic } \\
\text { properties }\end{array}$} & \multirow[b]{3}{*}{ ref } \\
\hline & \multicolumn{2}{|c|}{ AL-FS(FO) } & \multicolumn{2}{|c|}{ AL-DS(PRO) } & \multirow[b]{2}{*}{$\begin{array}{r}\text { Feed } \\
\text { solution }\end{array}$} & \multirow[b]{2}{*}{$\begin{array}{r}\text { Draw } \\
\text { solution }\end{array}$} & \multirow[b]{2}{*}{ cross-flow velocity } & & \\
\hline & $\begin{array}{r}\mathrm{J}_{\mathrm{v}} \\
(\mathrm{LMH})\end{array}$ & $\begin{array}{l}\mathrm{J}_{\mathrm{s}} / \mathrm{J}_{\mathrm{v}} \\
(\mathrm{g} / \mathrm{L})\end{array}$ & $\begin{array}{r}\mathrm{J}_{\mathrm{v}} \\
(\mathrm{LMH})\end{array}$ & $\begin{array}{l}\mathrm{J}_{\mathrm{s}} / \mathrm{J}_{\mathrm{v}} \\
(\mathrm{g} / \mathrm{L})\end{array}$ & & & & & \\
\hline PES & & & \multirow{3}{*}{15.79} & \multirow{3}{*}{0.26} & \multirow{3}{*}{$\begin{array}{c}1,000 \\
\text { ppm of } \\
\text { oil } \\
\text { emulsion }\end{array}$} & \multirow{3}{*}{$2 \mathrm{M} \mathrm{NaCl}$} & \multirow{3}{*}{$32.72 \mathrm{~cm} / \mathrm{s}$} & & \multirow{3}{*}[113]{} \\
\hline zwitterion & & & & & & & & $\begin{array}{c}\mathrm{A}=0.5 / \mathrm{LMH} / \mathrm{bar} \\
\mathrm{B}=0.45 \mathrm{LMH}\end{array}$ & \\
\hline PA & & & & & & & & $\mathrm{R}=95.8$ & \\
\hline PSF & \multirow{3}{*}{25.5} & \multirow{3}{*}{0.19} & & & \multirow{3}{*}{ DI water } & \multirow{3}{*}{$2 \mathrm{M} \mathrm{NaCl}$} & \multirow{3}{*}{$8.5 \mathrm{~cm} / \mathrm{s}$} & $\mathrm{A}=2.44 \mathrm{LMH} / \mathrm{bar}$ & \multirow{3}{*}{114} \\
\hline PA & & & & & & & & $\mathrm{B}=0.42 \mathrm{LMH}$ & \\
\hline $\mathrm{CaCl}_{2}$ & & & & & & & & $\mathrm{R}=97.9$ & \\
\hline cellulose acetate & \multirow{4}{*}{16.8} & \multirow{4}{*}{0.35} & \multirow{4}{*}{27.5} & \multirow{4}{*}{0.38} & \multirow{4}{*}{ DI water } & \multirow{4}{*}{$1 \mathrm{M} \mathrm{NaCl}$} & \multirow{4}{*}{$1666 \mathrm{ml} / \mathrm{min}$} & $\mathrm{A}=1.08 \mathrm{LMH} / \mathrm{bar}$ & \multirow{4}{*}{ [115] } \\
\hline butyrate $(\mathrm{CAB})$ and & & & & & & & & $\mathrm{B}=0.529 \mathrm{LMH}$ & \\
\hline PVB & & & & & & & & $\mathrm{S}=363.5 \mu \mathrm{m}$ & \\
\hline PA & & & & & & & & $\mathrm{R}=86.6$ & \\
\hline PSF & \multirow{2}{*}{73.87} & \multirow[b]{2}{*}{0.11} & \multirow[b]{2}{*}{120.1} & \multirow[b]{2}{*}{0.1} & \multirow[b]{2}{*}{ DI water } & \multirow[b]{2}{*}{$2 \mathrm{M} \mathrm{NaCl}$} & \multirow[b]{2}{*}{$300 \mathrm{ml} / \mathrm{min}$} & $\mathrm{A}=3.62 \mathrm{LMH} / \mathrm{bar}$ & \\
\hline $\begin{array}{c}\text { PA* (under }^{*} \\
\text { ultrasonication) }\end{array}$ & & & & & & & & $\begin{array}{c}\mathrm{B}=0.16 \mathrm{LMH} \\
\mathrm{R}=97.1\end{array}$ & [116] \\
\hline
\end{tabular}

${ }^{*}$ The majority of PA active layers that presented in table 1 were fabricated with conventional interfacial polymerization that was explained in section 2.2 except the ones have been highlighted by asterisk. 


\section{4. $\quad$ Performance of TFC-A Hollow fiber membranes}

3 Hollow fiber (HF) membranes can be more suitable for FO process than thin flat sheet membranes 4 because of their self-supported mechanical properties, since it allows denser packing density and 5 higher effective membrane surface area for both feed and draw solutions [117]. Moreover, 6 fabricating the module of the hollow fibers is relatively easy and the module provides greater 7 surface area per volume ratio. It should also be noted that the structure of hollow fibers may offer 8 the flow pattern specifically required for FO processes [117-121]. Nonetheless, hollow fiber 9 membranes manufacturing techniques suffer from a few challenges, such as restriction of 10 appropriate materials, i.e. hydrophilic and tough materials, as well as necessity to optimize the 11 phase separation techniques for the fabrication of hollow fibers with enhanced mechanical quality 12 and separation properties, while maintaining enough low structural parameter [122].

13 Fig. 8 shows schematically a dry-jet wet spinning of hollow fiber membranes. Readers can refer to the literature for the more details.

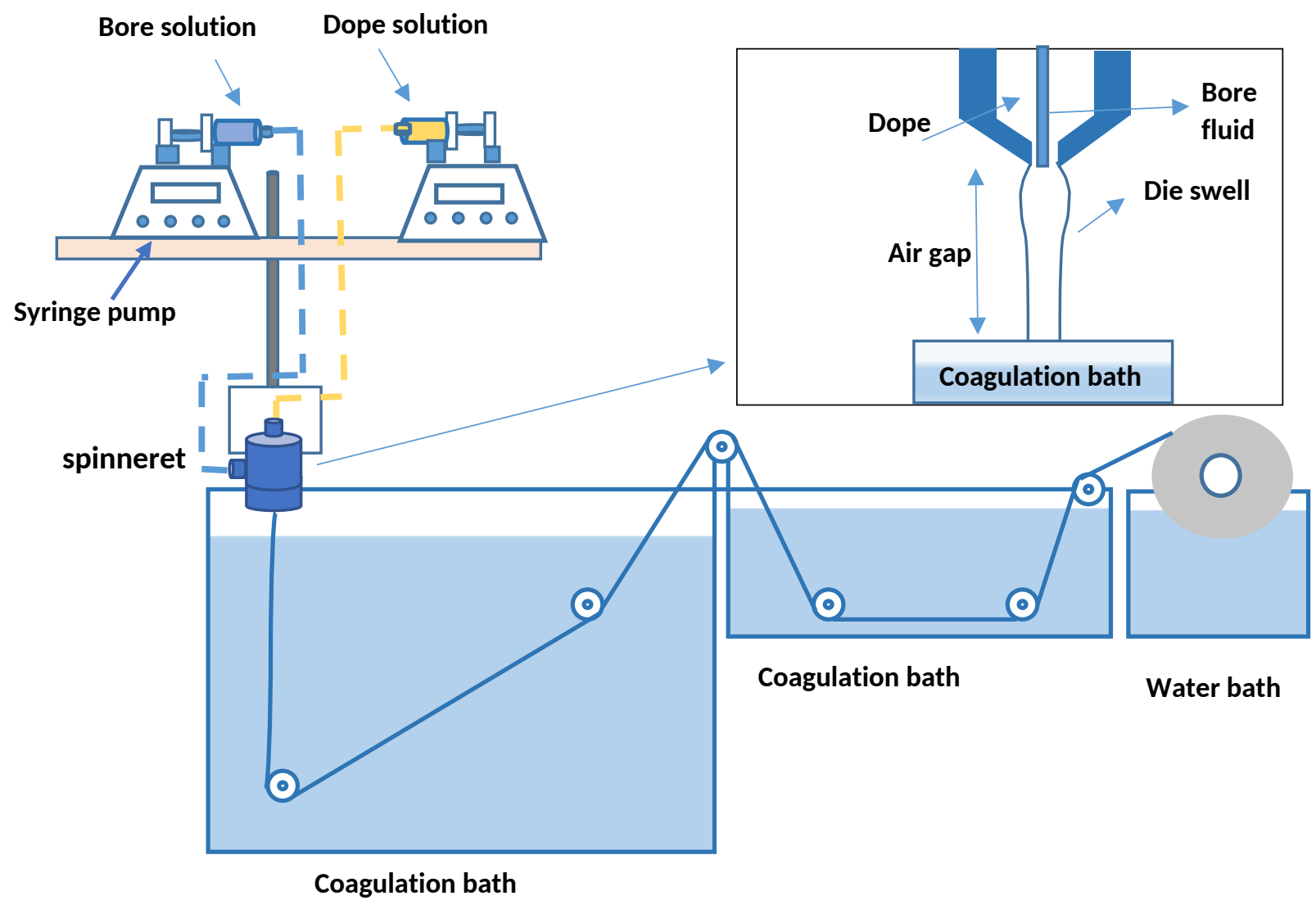

Fig 8: Schematic of dry-jet wet-spinning of hollow fiber membranes 


\subsubsection{PES as substrate}

Wang et al. [119] produced TFC-A hollow fiber membranes with PES substrates and compared them with commercial HTI flat sheet membranes and also commercial NF hollow fiber membranes when applied in an FO unit. In their experiments, two substrates, A and B, were spun. For A, a mixture of water and NMP was used as the bore fluid and tap water was used as the external coagulant, while for B only water was used for both internal and external coagulant. The active layer was synthesized onto the outer surface of the substrate A while the active layer was synthesized in the inner surface of substrate B. Both substrates had a sponge-like and a porous structure. However, the contribution of the sponge-like structure was lower in substrate B, and hence B exhibited a higher water flux. They have considered that the structural parameter, $S$, of the membrane is a series combination of the sponge-like and porous structure, as shown in Eq. (1), with the sponge-like structure of greater contribution to $S$. Since the substrate B has smaller sponge-like fraction, smaller $S$ and less internal concentration polarization, the substrate B is preferable. However, they did not explain, it can be found out from the SEM results, that the "pore" here refers to the finger-like structure.

$$
S=\frac{\tau_{\text {sponge }} l_{\text {sponge }}}{\varepsilon_{\text {sponge }}}+\frac{\tau_{\text {pore }} l_{\text {pore }}}{\varepsilon_{\text {pore }}}
$$

Furthermore, they have discussed on the bore shrinkage of the substrate membrane B during the hollow fiber drying, which increases the fluid flow resistance in the bore. It could be prevented by replacing the water in the membrane pore with water/glycerol mixture before drying. The optimal membrane made in this study (membrane B without bore shrinkage) showed a better performance compared to the HTI commercial flat sheet membrane.

Shi et al. [123] also studied the effect of the pore size of the hollow fiber substrate on the active layer properties of TFC membranes and their FO performance. After PES hollow fiber substrates were spun by the dry-jet wet method, polyamide active layer was formed onto the lumen side of substrates. They found that defective active layer is formed more readily when the substrate pore size is large. This result coincides with the earlier study of Singh et al. [91] on the mechanism of the active layer formation using two substrates with different pore sizes. Fig. 9 shows schematically the skin layer formation mechanism proposed by Singh et al. [91] For type1 substrate with smaller pore sizes, monomer does not penetrate into the pore easily and thus the polyamide active layer is formed only onto the substrate surface. However, in the case of the substrate type 2 with larger pore sizes, monomer penetrates into the substrate pore and thus a part of the active layer is formed inside the pore, reducing the active layer thickness above the substrate surface and thus increasing the chance of defect formation. 


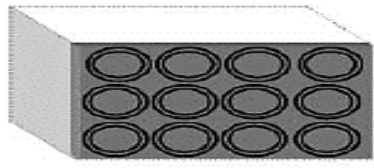

Front view

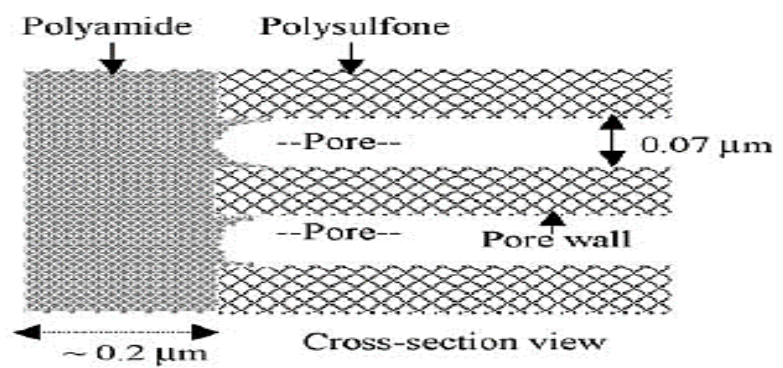

Type 1

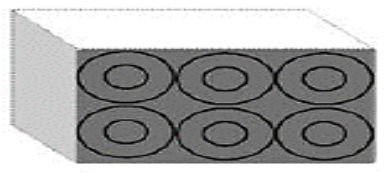

Front view

Fig 9: Formation of polyamide layer on the poly sulfone layer for two substrates with different pore sizes [91]

Sukitpaneenit and Chung [124] produced a polyamide TFC hollow fiber membrane with PES substrate using a two-layer coextrusion, as shown in Fig. 10. From the figure, the nonsolvent bore fluid (either (a) water, (b) water/NMP, or (c) water/NMP/PEG), polymer dope and NMP are extruded from the central tube, the inner channel and the external channel, respectively. The water containing bore fluid facilitates the phase inversion process, thus forming a dense skin layer at the inner surface, while delayed phase inversion caused by NMP makes the outer surface more porous. Hence the hollow fiber membrane shows an asymmetric structure as shown in Fig. 11. The polymer dope consisted of PES, NMP, water, and PEG. Addition of water increases the dope viscosity and also brings the dope composition near the binodal line, both contributing to the prevention of macro voids formation. However, PEG acts as a pore former, it also prevents the formation of macro voids. The internal surface roughness is controlled by the property of the internal coagulant, e.g. instantaneous demixing caused by water makes the internal surface the roughest among the three different internal coagulants. In addition, the membrane contained a mixture of finger-like structure, sponge-like structure and macro voids (not shown in Fig. 11).

In the earlier works $[119,125]$, it has been stated that an ideal substrate for TFC hollow fiber membranes should include more finger-like structure and large pores even though some small parts of the cross-section are allowed to be occupied by sponge-like structure. However in this work [124], even though a large part of the substrate is occupied by sponge-like structure instead of finger-like pores (see Fig. 11), this substrate was found equally suitable to reduce ICP and to achieve a high water flux. 


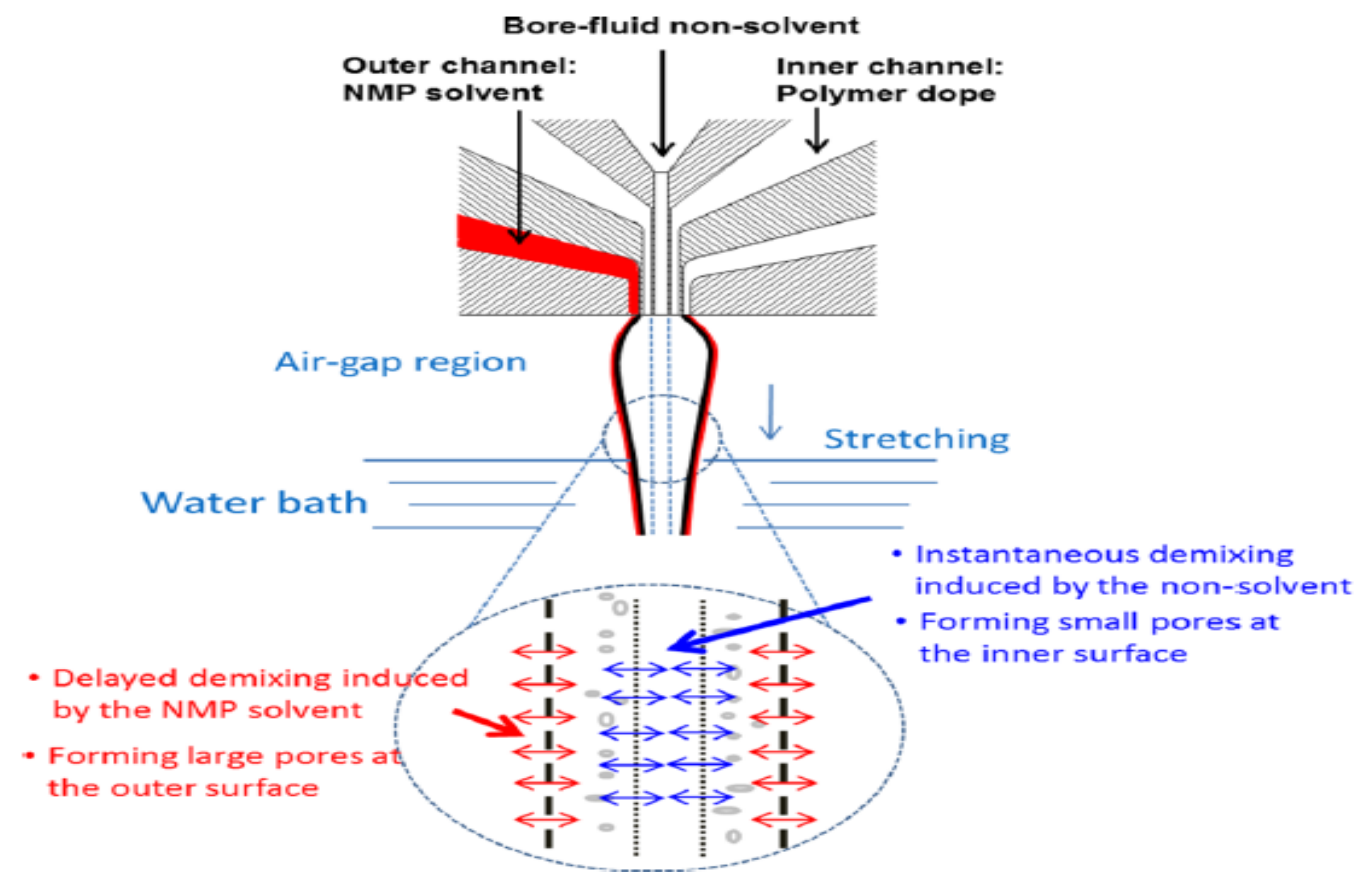

Fig 10: Phase inversion process with the aid of coextrusion technique employing a dual-layer spinneret [124] 


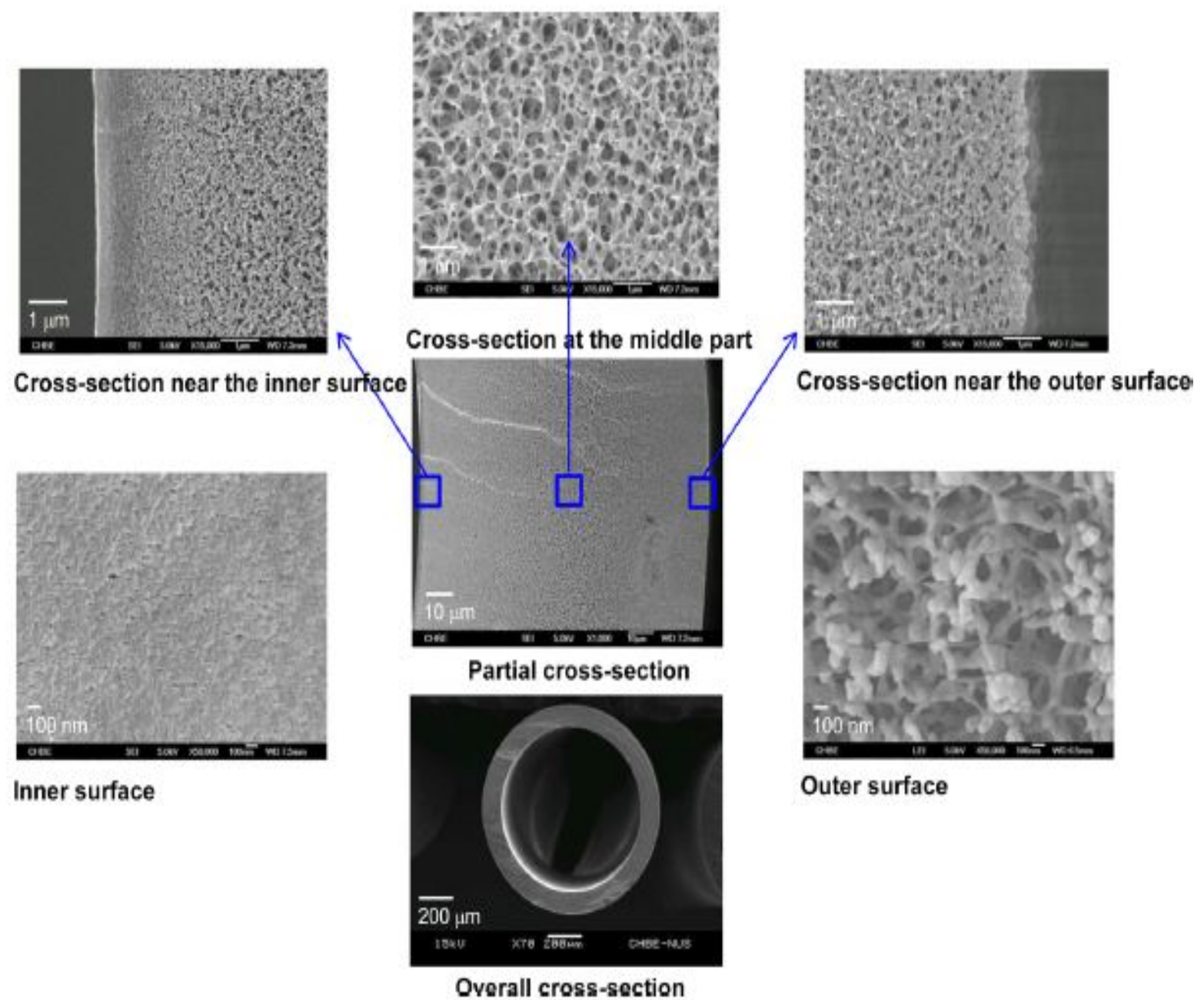

Fig 11: SEM micrographs of different bulk and surface morphologies of PES hollow fiber membrane supports [124]

Lim et al. [126] fabricated a defect free outer selective layer hollow fiber membrane with PES substrate. The advantages of making the active layer on the outer surface of the substrate are i) it provides a larger surface area per fiber and ii) it offers an easier fouling control under FO mode and lower fouling propensity. Also, they studied the effect of air gap on the performance of the FO membranes. The air-gap distance was in the range of $2-8 \mathrm{~cm}$. At the lowest air-gap the membrane substrate showed the highest porosity, the largest fiber diameter and the largest mean pore size compared to the other samples. These properties (with the lowest air gap) related to low elongation stress on the fibers in the air-gap region. Thus, a thicker and rougher active layer formed on the substrate with lowest air gap. Eventually, a defective active layer was formed on this substrate. When the surface pore size is large, the MPD solution cannot fill the pores uniformly and can be diffuse inside the pores or may be removed by air-blowing because of weak surface tension. These phenomena have also been reported by the other researchers [91, 123]. 


\subsubsection{Polyphenylene sulfone as substrate}

Zhong et al. [120] studied the effects of degree of sulfonation of a polyphenylene sulfone substrate on the performance of hollow fiber polyamide TFC-A membrane. In this study, the active layer was synthesized onto the membrane lumen surface. The advantages of making the active layer onto the inner surface of substrate are i) a better distribution of feed when feed flows through the lumen side, ii) easy protection of the defect-free active layer and, iii) low resistance against water permeation. Also, when feed flows in the lumen side, no dead space is created. On the other hand, when the feed flows in the shell side, the non-ideal fluid passage leads to creation of dead spaces, hence severe concentration polarization is experienced [127]. Eventually, Zhong et al. [120] obtained the following results:

1) By increasing the degree of sulfonation, i.e. the higher the sulfonate concentration in the substrate, the substrate tended toward sponge-like structure formation and the thickness of the hollow fiber increased due to the increase in viscosity i.e. delayed demixing. Conversely, the lower the sulfonate concentration in the substrate, the less viscosity of the dope solution. As a result, faster precipitation was experienced and larger pores were formed.

2) Degree of sulfonation showed a direct correlation to the membrane hydrophilicity.

3) As the degree of sulfonation increased, the water flux increased as well. Because the sulfonation tended to encourage the formation of sponge-like structure, it was concluded that the effect of hydrophilicity on flux was more pronounced than the effect of structure as mentioned earlier by other researchers [81]. $3.5 \% \mathrm{NaCl}$ solution as feed and 2 molar $\mathrm{NaCl}$ solution as draw solution in AL-DS (PRO) orientation using a membrane with a sulfonate content. By reducing the fibers lengths, the effect of internal and external concentration polarization decreased and therefore the water flux was increased. The effect of the fiber length on reverse salt flux was not reported in this study; however, the effect of the fiber length on the membrane performance is a controversial issue that needs further investigation.

\subsubsection{Matrimid $^{\circledR}$ as substrate}

Luo et al. [121] designed a new TFC-A hollow fiber membrane consisting of Matrimid ${ }^{\circledR}$ substrate and active polyamide layer for FO. Matrimid ${ }^{\circledR}$ is a commercial aromatic polyimide that is soluble in common organic solvents [129]. The active layer was made, again, onto the inner surface of the membrane. The spinneret geometry had a blossom geometry with three separate bore flows (Fig. 12). The tri-needle spinneret had three separate needles placed inside it uniformly. This special spinneret design was made to address the concern over the long-term stability and durability of the potting (seal) of hollow fibers spun from the single-needle spinneret. Indeed, the hollow fibers spun by a multi bore spinneret showed better durability during the long term operations and mechanical washing [130]. Fig. 13 schematically shows the two different hollow fiber cross sections. Between those, triangular geometry (Fig. 13 left) was suggested for balancing the mechanical resistance and water flux. The triangular geometry also showed better module 
performance because of the higher packing density, effective surface area of membrane and water flux. In contrast to triangular geometry, circular geometry shows disadvantages such as the uneven thickness of the walls, low mechanical resistance of the thin walls, and high-water permeation resistance of the thick walls. The key parameters in creating the triangular geometry were closeto-gelation dope formation, the flow rate of the dope solution, the air gap, and the characteristics of the bore fluid and the coagulant [121]. Despite the advantages they mentioned, when feed flows in the shell side, triangular shape may result in severe fouling between the fibers.
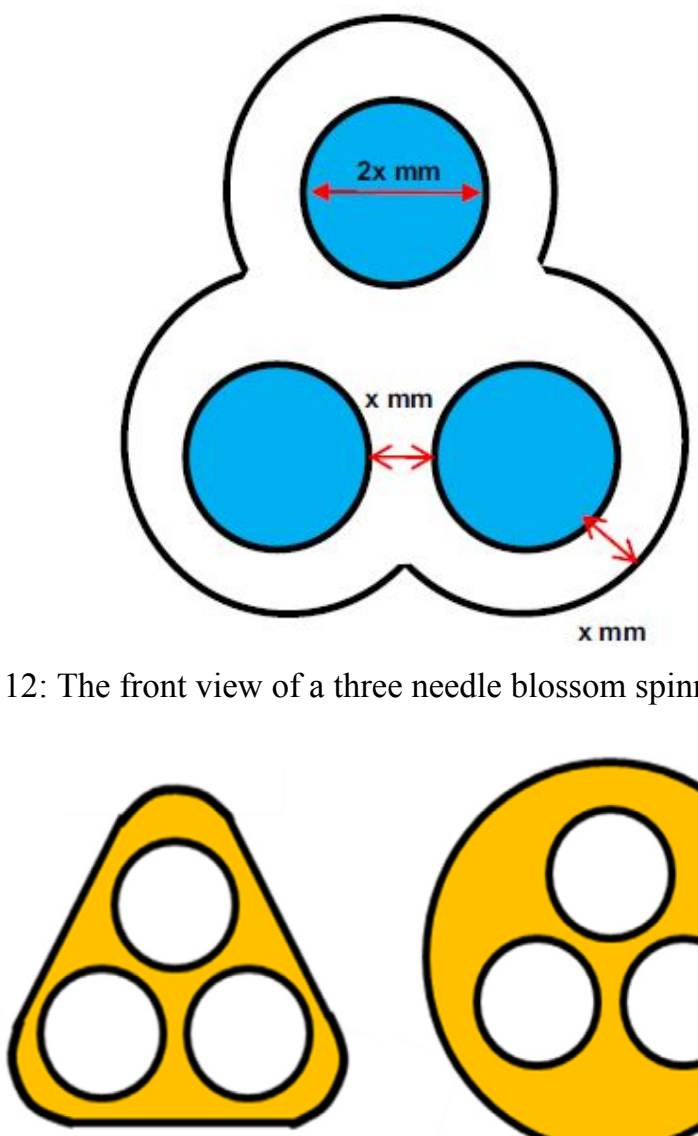

Fig 12: The front view of a three needle blossom spinneret [130]

Ren et al. [131] produced a TFC hollow fiber membranes with PAN substrate and polyamide active layer. PAN spins easily and is inherently hydrophilic. In contrast to the other works, the polyamide active layer was at the outer surface, which showed less fouling tendency compared to the membranes in which the active layer was at the inner surface. Usually, it is more difficult to form

21 the inner surface of hollow fiber, especially for large-scale production. Conversely, Zhong [120] 
outer surface. The selection of inner or outer selective layer can be related to the operation condition, i.e. the type of feed and/or draw solutions. It is well known that the formation of defect free active layer depends on how well the aqueous MPD solution is removed before the substrate is immersed in the organic phase. In the case of the flat sheet membrane, aqueous solution is removed by a roller. The aqueous solution in the lumen of hollow fiber can be blown by air. To form the active layer onto the outer surface of hollow fiber, the interfacial polymerization should be carried out continuously, i.e. the hollow fiber should pass through aqueous solution, air, and organic solution chamber progressively. The method has some problems, namely (i) the membrane outer surface contacts with the moving surface of the pulley during the interfacial polymerization creating an imperfect layer. (ii) the continuous flow of thousands of meters of fibers in large-scale production is time-consuming. (iii) because of many controlling parameters the process is complex. Therefore, the active layer formation onto the outer surface was proposed to be done not for a single fiber but for multiple of hollow fibers all at once [118]. But even in this case, the interfacial polymerization may take place between two fibers, if the aqueous solution is not well removed.

In Ren et al. [131] work, a batch method was used. Three substrate hollow fibers were spun with the bore fluids of three different DMF contents, ranging from 0 to $60 \%$, in the DMF/water mixture. In Fig. 14, the cross-sectional FESEM images of the substrates are shown with two different magnifications. All the three substrates had a flat and smooth surface that was necessary for forming strong and defect free active layer. As DMF concentration in bore fluid increases, the rate of polymer solidification during the phase inversion is delayed, resulting in thinner hollow fiber wall with more finger-like pores.

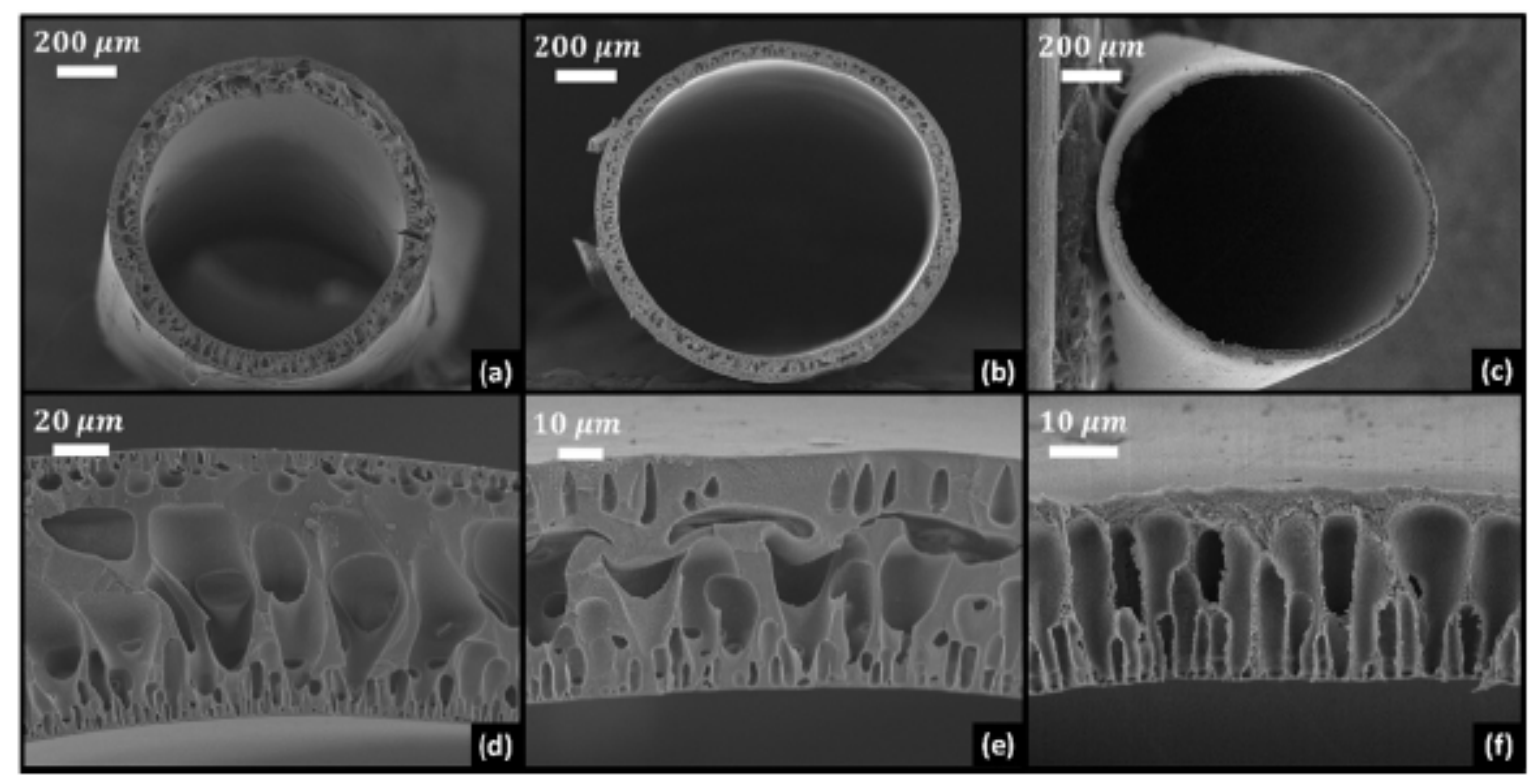

Fig 14: Cross-sectional FESEM images of hollow fiber substrates. (a), (b) and (c) PAN-0, PAN-30 and PAN-60 at $65 \times$, respectively. (d) PAN-0 at 600×, (e) PAN-30 at 950×, (f) PAN-60 at 1000× [131]

\subsubsection{Polyketone as substrate}


Shibuya et al. [132] prepared TFC-A hollow fiber membranes on the membrane performance with two different substrates, both made of Polyketone. Polyketone possesses good mechanical and chemical properties and shows good behavior over a wide range of temperature. As a drawback, polyketone is not dissolved in organic solvents [133]. They formed the active layer on the outer surface by successive immersion of hollow fibers in the aqueous and organic solutions (see Fig. 15). Both ends of the substrates were sealed by epoxy resin to prevent the solution from entering 7 into the lumen side of the membranes [132].

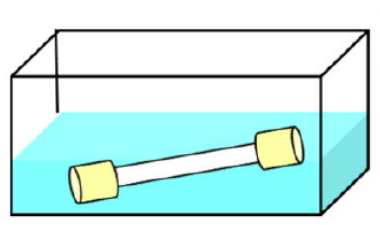

Immersion in Aqueous MPD solution

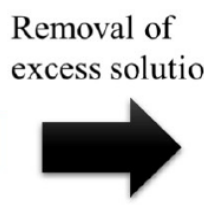

(1)

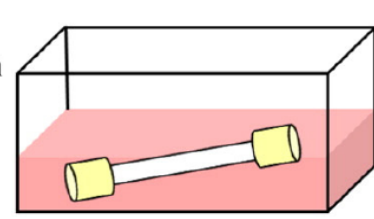

Immersion in Hexane TMC solution

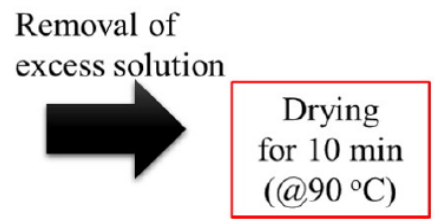

(@, $\left.90^{\circ} \mathrm{C}\right)$

Fig 15: Steps of the interfacial polymerization at the shell side of the hollow fiber membranes [132]

10

11

12

13

14

15

As shown in Table 2, the two substrates, HF-A and HF-B, were very different in diameters and thicknesses but similar in mean pore size and porosity and both possessed microfiltration structure [132].

Table 2: HF support membrane characteristics [132]

\begin{tabular}{|c|c|c|c|c|c|}
\hline sample & $\begin{array}{l}\text { Inner diameter } \\
(\mu \mathrm{m})\end{array}$ & $\begin{array}{l}\text { Outer diameter } \\
\qquad(\mu \mathrm{m})\end{array}$ & $\begin{array}{c}\text { Thickness } \\
(\mu \mathrm{m})\end{array}$ & $\begin{array}{l}\text { Mean pore size } \\
(\mathrm{nm})\end{array}$ & $\begin{array}{l}\text { Porosity } \\
(\%)\end{array}$ \\
\hline HF-A & 347 & 480 & 66.5 & 41.4 & 73.6 \\
\hline HF-B & 609 & 893 & 142 & 47.8 & 78.0 \\
\hline
\end{tabular}

Water flux and reverse salt flux of HF-A membrane were higher than those of HF-B membrane despite the smaller porosity of HF-A. This is because of the thinner wall of HF-A. As the draw solution concentration increased both water flux and reverse salt flux increased but specific reverse salt flux remained unchanged. They stated that, this refers to the fact that, specific reverse salt flux is an intrinsic property of the active layer [132].

The works published in the literature on hollow fiber TFC-A membranes are summarized in Table 3. The table includes water flux and specific reverse salt flux with a polyamide active layer in the two different modes of AL-FS(FO) and AL-DS(PRO). 
Table 3: Summary of the studies on TFC-A hollow fiber FO membranes

\begin{tabular}{|c|c|c|c|c|c|c|c|c|c|c|}
\hline \multirow{3}{*}{$\begin{array}{c}\text { support } \\
\text { Active layer } \\
\text { Active layer place }\end{array}$} & \multicolumn{4}{|c|}{ Performance } & \multicolumn{4}{|c|}{ Experimental condition } & \multirow{3}{*}{$\begin{array}{c}\text { Intrinsic } \\
\text { properties }\end{array}$} & \multirow{3}{*}{ ref } \\
\hline & \multicolumn{2}{|c|}{ AL-FS(FO) } & \multicolumn{2}{|c|}{ AL-DS(PRO) } & \multirow{2}{*}{$\begin{array}{c}\text { Feed } \\
\text { solution }\end{array}$} & \multirow{2}{*}{$\begin{array}{r}\text { Draw } \\
\text { solution }\end{array}$} & \multirow{2}{*}{$\begin{array}{c}\text { cross-flow velocity } \\
\text { or } \\
\text { Reynolds number } \\
\text { Lumen side }\end{array}$} & \multirow{2}{*}{$\begin{array}{c}\text { cross-flow velocity } \\
\text { or } \\
\text { Reynolds number } \\
\text { Shell side }\end{array}$} & & \\
\hline & $\begin{array}{r}\mathrm{J}_{\mathrm{v}} \\
(\mathrm{LMH})\end{array}$ & $\begin{array}{l}\mathrm{J}_{\mathrm{s}} / \mathrm{J}_{\mathrm{v}} \\
(\mathrm{g} / \mathrm{L})\end{array}$ & $\begin{array}{r}\mathrm{J}_{\mathrm{v}} \\
(\mathrm{LMH})\end{array}$ & $\begin{array}{l}\mathrm{J}_{\mathrm{s}} / \mathrm{J}_{\mathrm{v}} \\
(\mathrm{g} / \mathrm{L})\end{array}$ & & & & & & \\
\hline $\begin{array}{c}\text { PES } \\
\text { PA } \\
\text { inner surface }\end{array}$ & 14 & 0.13 & 32.2 & 0.11 & DI water & $\begin{array}{r}0.5 \mathrm{M} \\
\mathrm{NaCl}\end{array}$ & 1500 & 450 & $\begin{array}{c}\mathrm{A}=2.22 \mathrm{LMH} / \mathrm{bar} \\
\mathrm{B}=0.2 \mathrm{LMH} \\
\mathrm{S}=5.95 \times 10^{-4} \mathrm{~m} \\
\mathrm{R}=91\end{array}$ & {$[119]$} \\
\hline $\begin{array}{c}\text { PES } \\
\text { PA } \\
\text { inner surface } \\
\end{array}$ & 18.3 & 0.086 & 42.6 & 0.094 & DI water & $\begin{array}{lc}0.5 & \mathrm{M} \\
& \mathrm{NaCl}\end{array}$ & 2500 & 2500 & $\begin{array}{c}\mathrm{A}=3.2 \mathrm{LMH} / \mathrm{bar} \\
\mathrm{R}=92\end{array}$ & {$[125$} \\
\hline $\begin{array}{c}\text { PES } \\
\text { PA } \\
\text { inner surface }\end{array}$ & 17.6 & 0.068 & 49 & 0.081 & DI water & $\begin{array}{cc}0.5 & \mathrm{M} \\
& \mathrm{NaCl}\end{array}$ & $450 \mathrm{~mL} / \mathrm{min}$ & $1500 \mathrm{~mL} / \mathrm{min}$ & $\begin{array}{c}\mathrm{A}=3.93 \mathrm{LMH} / \mathrm{bar} \\
\mathrm{B}=0.27 \mathrm{LMH} \\
\mathrm{S}=0.51 \mathrm{~mm} \\
\mathrm{R}=90.4\end{array}$ & {$[123$} \\
\hline $\begin{array}{c}\text { PES } \\
\text { PA } \\
\text { inner surface }\end{array}$ & 32.1 & 0.2 & 57.1 & 0.12 & DI water & $2 \mathrm{M} \mathrm{NaCl}$ & $100 \mathrm{ml} / \mathrm{min}$ & $200 \mathrm{ml} / \mathrm{min}$ & $\begin{array}{c}\mathrm{A}=1.18 \mathrm{LMH} / \mathrm{bar} \\
\mathrm{B}=0.135 \mathrm{LMH} \\
\mathrm{S}=2.19 \times 10^{-4} \mathrm{~m} \\
\mathrm{R}=87.95\end{array}$ & {$[124$} \\
\hline $\begin{array}{c}\begin{array}{c}\text { sulfonated poly } \\
\text { phenylene sulfone } \\
\text { (sPPSU) }\end{array} \\
\text { PA } \\
\text { inner surface } \\
\end{array}$ & 22.51 & 0.24 & 49.39 & 0.22 & DI water & $\begin{array}{cc}0.5 & \mathrm{M} \\
& \mathrm{NaCl}\end{array}$ & $100 \mathrm{ml} / \mathrm{min}$ & $200 \mathrm{ml} / \mathrm{min}$ & $\begin{array}{c}\mathrm{A}=1.99 \mathrm{LMH} / \mathrm{bar} \\
\mathrm{B}=0.0399 \mathrm{LMH} \\
\mathrm{S}=1.63 \times 10^{-4} \mathrm{~m} \\
\mathrm{R}=90.9\end{array}$ & {$[120$} \\
\hline $\begin{array}{c}\text { PES } \\
\text { PA } \\
\text { cetyltrimethylammonium } \\
\text { chloride } \\
\text { (CTAC) }\end{array}$ & 5.32 & & & & DI water & $2 \mathrm{M} \mathrm{NaCl}$ & & & $\mathrm{R}=98$ & {$[134$} \\
\hline Outer surface & & & & & & & & & & \\
\hline $\begin{array}{c}\text { Matrimid } \\
\text { PA } \\
\text { inner surface }\end{array}$ & 11.8 & 0.21 & 50.5 & 0.07 & DI water & $2 \mathrm{M} \mathrm{NaCl}$ & $200 \mathrm{ml} / \mathrm{min}$ & $200 \mathrm{ml} / \mathrm{min}$ & $\begin{array}{c}\mathrm{A}=1.51 \mathrm{LMH} / \mathrm{bar} \\
\mathrm{B}=0.44 \mathrm{LMH} \\
\mathrm{S}=1.1 \times 10^{-3} \mathrm{~m}\end{array}$ & {$[121$} \\
\hline
\end{tabular}


Table 3: (continued)

\begin{tabular}{|c|c|c|c|c|c|c|c|c|c|c|}
\hline \multirow{3}{*}{$\begin{array}{c}\text { support } \\
\text { Active layer } \\
\text { Active layer place }\end{array}$} & \multicolumn{4}{|c|}{ Performance } & \multicolumn{4}{|c|}{ Experimental condition } & \multirow{3}{*}{$\begin{array}{l}\text { Intrinsic } \\
\text { properties }\end{array}$} & \multirow{3}{*}{ ref } \\
\hline & \multicolumn{2}{|c|}{ AL-FS(FO) } & \multicolumn{2}{|c|}{ AL-DS(PRO) } & \multirow{2}{*}{$\begin{array}{c}\text { Feed } \\
\text { solution }\end{array}$} & \multirow{2}{*}{$\begin{array}{r}\text { Draw } \\
\text { solution }\end{array}$} & \multirow{2}{*}{$\begin{array}{l}\text { cross-flow velocity } \\
\text { or } \\
\text { Reynolds number } \\
\text { Lumen side }\end{array}$} & \multirow{2}{*}{$\begin{array}{c}\text { cross-flow velocity } \\
\text { or } \\
\text { Reynolds number } \\
\text { Shell side }\end{array}$} & & \\
\hline & $\begin{array}{r}\mathrm{J}_{\mathrm{v}} \\
(\mathrm{LMH})\end{array}$ & $\begin{array}{l}\mathrm{J}_{\mathrm{S}} / \mathrm{J}_{\mathrm{v}} \\
(\mathrm{g} / \mathrm{L})\end{array}$ & $\begin{array}{r}\mathrm{J}_{\mathrm{v}} \\
(\mathrm{LMH})\end{array}$ & $\begin{array}{l}\mathrm{J}_{\mathrm{s}} / \mathrm{J}_{\mathrm{v}} \\
(\mathrm{g} / \mathrm{L})\end{array}$ & & & & & & \\
\hline PAN & \multirow{3}{*}{24.71} & \multirow{3}{*}{0.77} & \multirow{3}{*}{36.57} & \multirow{3}{*}{0.512} & \multirow{3}{*}{ DI water } & \multirow{3}{*}{$1 \mathrm{M} \mathrm{NaCl}$} & \multirow{3}{*}{1100} & \multirow{3}{*}{\multicolumn{2}{|c|}{$\begin{array}{c}\mathrm{A}=1.5 \mathrm{LMH} / \mathrm{bar} \\
\mathrm{B}=2.1 \mathrm{LMH}\end{array}$}} & \multirow{3}{*}{ [131] } \\
\hline PA & & & & & & & & & & \\
\hline outer surface & & & & & & & & & & \\
\hline PA & & & \multirow[t]{2}{*}{52} & \multirow[t]{2}{*}{0.22} & \multirow[t]{2}{*}{ DI water } & \multirow[t]{2}{*}{$1 \mathrm{M} \mathrm{NaCl}$} & \multirow[t]{2}{*}{$15 \mathrm{~mL} / \mathrm{min}$} & & $\mathrm{B}=0.125 \mathrm{LMH}$ & \multirow[t]{2}{*}{ [132] } \\
\hline Outer surface & & & & & & & & & $\begin{array}{c}\mathrm{S}=334.1 \mu \mathrm{m} \\
\mathrm{R}=85.1\end{array}$ & \\
\hline PES & \multirow{3}{*}{30.2} & \multirow{3}{*}{0.13} & & & \multirow{3}{*}{ DI water } & \multirow{3}{*}{$1 \mathrm{M} \mathrm{NaCl}$} & & & $\mathrm{A}=2.26 \mathrm{LMH} / \mathrm{bar}$ & \multirow{3}{*}{ [126] } \\
\hline PA & & & & & & & & & $\mathrm{B}=0.28 \mathrm{LMH}$ & \\
\hline outer surface & & & & & & & & & $\begin{array}{c}S=190 \mu \mathrm{m} \\
\mathrm{R}=85.1\end{array}$ & \\
\hline
\end{tabular}




\subsection{Issues and implications of TFC-A membranes}

Fig. 16a illustrates specific salt flux $\left(J_{S} / J_{v}\right)$ versus water flux/ $\Delta \pi\left(J_{v} / \Delta \pi\right)$ as a summary of data reported in Table 1 and 3. In the figure, the red symbols show the performance of TFC-A membrane with nanofiber substrate fabricated via electro-spinning method. This type of membranes show more reasonable water flux and specific reverse salt flux in comparison to the phase inversion membranes, including both flat sheet and hollow fiber. As stated in section 2.1, although the mechanical strength of nanofiber layer is not high, it does not seem to be an important issue since FO is not a pressure driven membrane process. However, it may still become an important issue even for FO process for its large-scale and long-term operation. One way of increasing the mechanical strength is the support by a backing fabric. Unfortunately, however, the water flux could be compromised by adding a support layer [100]. Therefore, to find the methods to increase the mechanical strength of nanofiber substrate is recommended as a future research focus. It is evident that hollow fiber TFC membranes present better performance compared with flat sheet membranes, however their fouling propensity should be further investigated. The number of studies where the fabrication of hollow fiber membranes having active layers at the outer surface of the membrane is very limited. Nonetheless, because these membranes have less tendency toward fouling, they are more suitable for wastewater treatment than the inner skin. The majority of the hollow fiber membranes synthesized for the FO process are with a PES substrate. Although PES possesses good mechanical and chemical properties, its fouling resistance is low. From this aspect polymers other than PES with lower fouling tendency should be searched for to prepare the substrate [66]. Surface modification, such as by PDA coating of the flat sheet membrane prepared by the phase inversion method, showed a reasonable performance [92]. It should be emphasized that the morphology of substrate skin layer can affect the active layer during the IP step, resulting in unpredictable membrane performances [135]; however, this aspect needs further investigation. 


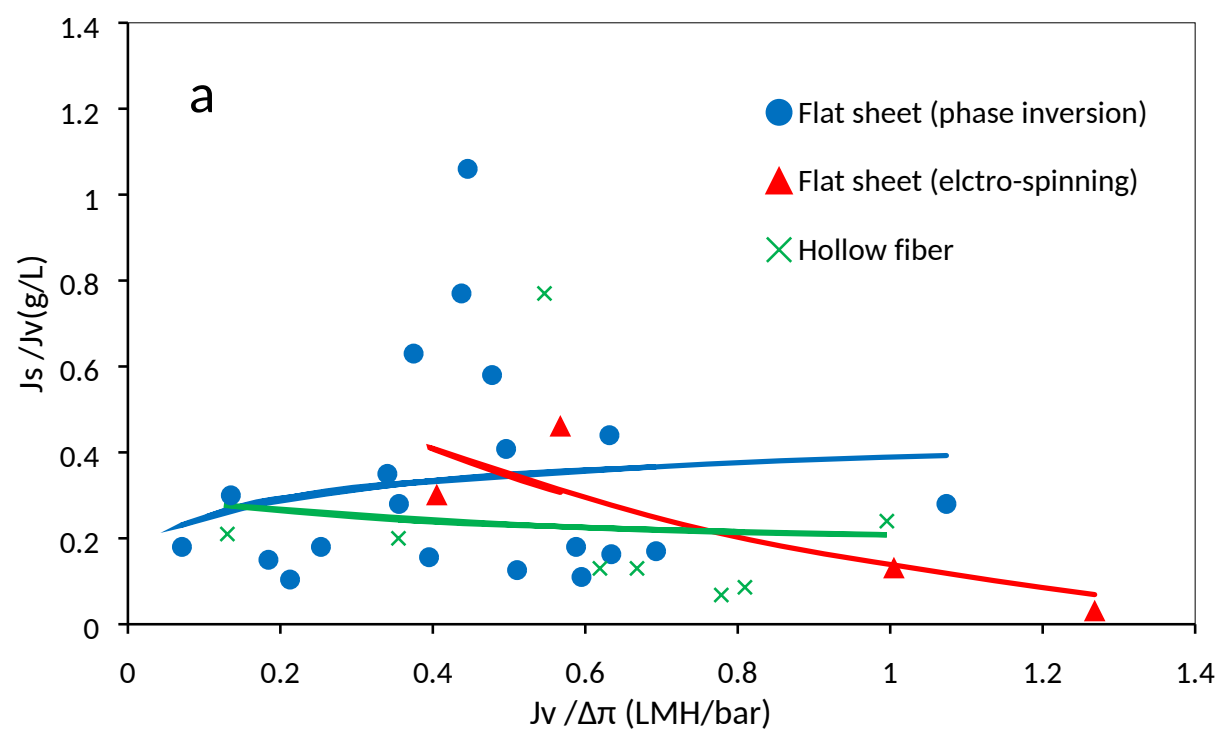

1

2

3

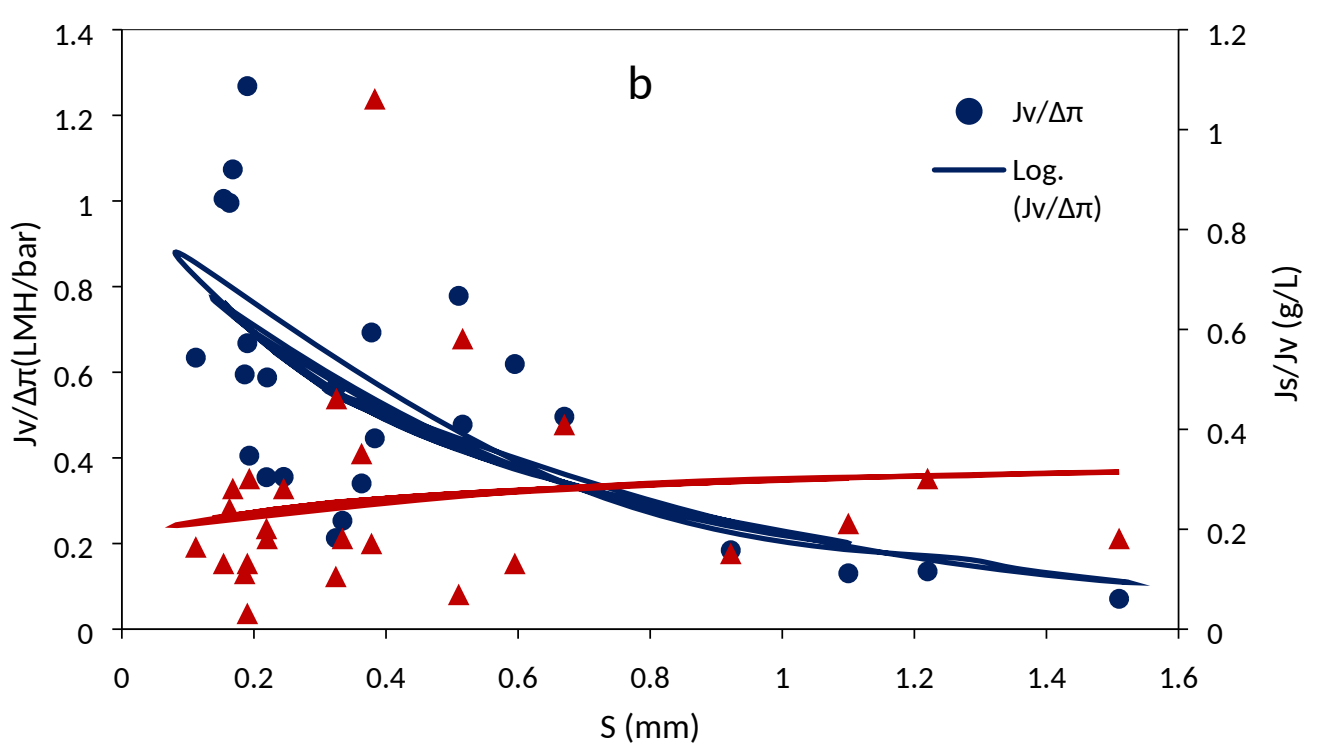

Fig 16: Water flux/driving force versus specific reverse a) specific reverse salt flux for and b) salt flux respectively for TFC-A FO membranes (the data point are taken from Tables 1 and 3)

Fig. 16b shows water flux $/ \Delta \pi\left(J_{v} / \Delta \pi\right)$ and specific reverse salt $\left(J_{S} / J_{v}\right)$ versus structural parameter. As evidenced, the structural parameter is an indication of internal concentration polarization [89]. For membrane processes with osmotic driving force, the lower the structural parameter, the higher the membrane performance $[100,136,137]$. It is because by increasing the structural parameter, 
the effective driving force across the membrane decreases. According to the theory, as shown in Figure 16b, with increasing $\mathrm{S}$, the trend of water flux changes is descending. However, the trend of specific reverse salt versus structural parameter is almost constant. The stability of these changes reveals a linear dependency of the salt permeability and water permeability coefficients. Because the separation properties of the active layer meaningfully depend on the properties of the sublayer, further investigation should be done to disclose the details.

\subsection{Performance of TFC-N membranes}

The addition of nanoparticles to substrate as well as to active layer of TFC membranes is one of the recent developments in this field that effectively changes the properties of resulted membrane. This technique affects the thickness, porosity, permeability, hydrophilicity, and roughness of substrate surface. It also influences the structure of active layer. Addition of nanomaterials in either aqueous or organic solution creates nano-scale pores in the polyamide active layer. Therefore, the addition of nanoparticles usually increases water flux but decreases the salt rejection [69, 138]. In addition, some nanoparticles have antifouling properties [40, 139-142]. In fact, addition of nanoparticles into substrate or active layer of a membrane can be considered as a kind of modification. The membrane modification approaches can be classified into two types, i.e., physical modification [143] and chemical modification [144]. Physical modification means that there is no chemical reaction among the components however in chemical modification chemical reaction occurs [65]. Lau et al. [145] reviewed the literature on making TFC-N membranes for different membrane processes. They identified several challenges in the fabrication of TFC-N membranes. Resolving these challenges can improve TFC-N membranes and make them suitable for a wide range of industrial applications. The main challenges are i) agglomeration of nanoparticles, due to their high surface energy, in the polyamide active layer, which reduces the surface area of nanoparticles as well as creates defects in the active layer. ii) loss of hydrophilic nanoparticles added to the aqueous solution at the time of removing the extra aqueous solution from the substrate surface. It would be better first to surface modify the hydrophilic nanoparticles and then add to the organic phase. iii) lack of chemical interaction between the polyamide active layer and nanoparticles. There are some solutions to these challenges, however, more studies are required to develop TFC-N membranes for industrial applications. In this section TFC-N membranes specified for FO process are reviewed in three classification as already depicted in Fig 1. In Fig. 17 schematic of different structure of TFC-N with their advantages and challenges are depicted: 
(a)

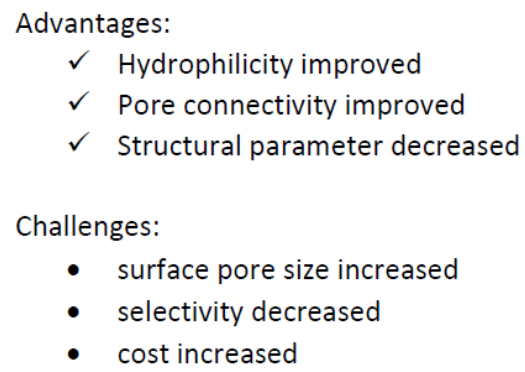

- $\quad$ surface pore size increased

- selectivity decreased

- cost increased

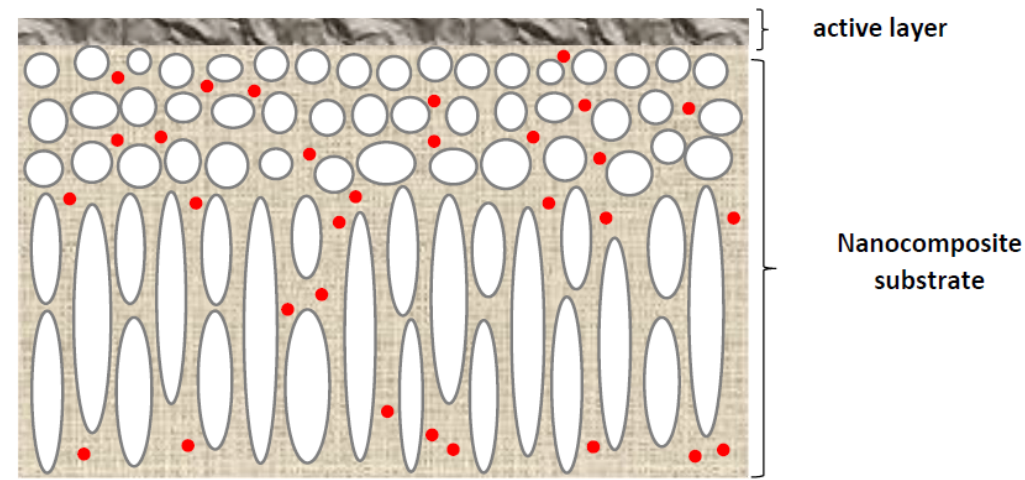

(b)

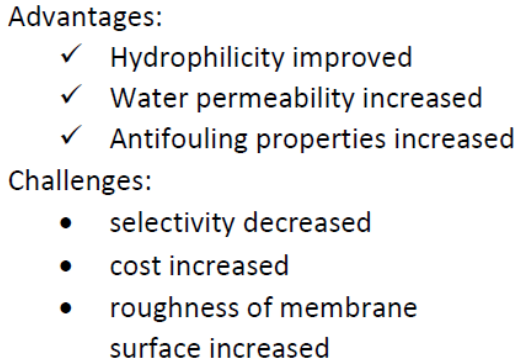

$\checkmark$ Hydrophilicity improved

$\checkmark$ Water permeability increased

$\checkmark$ Antifouling properties increased Challenges:

- selectivity decreased

- cost increased

- roughness of membrane surface increased

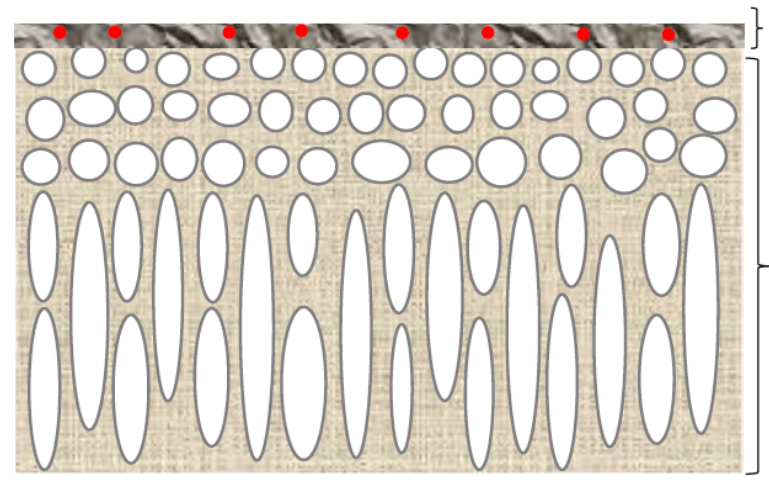

Nanocomposite active layer

substrate

(c)

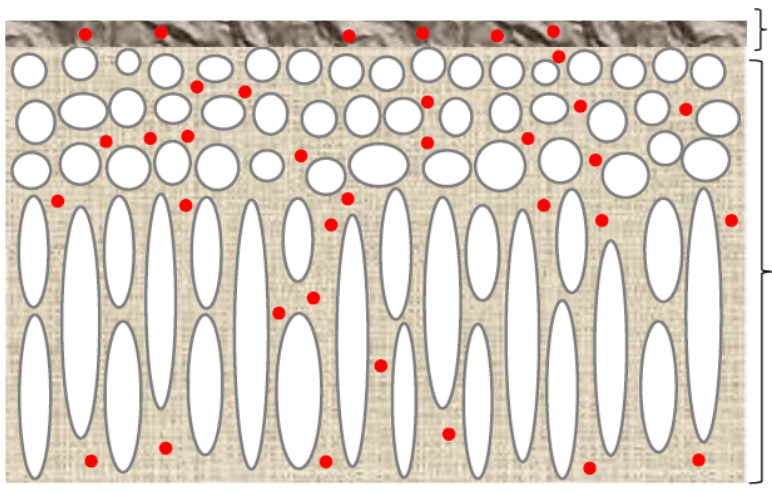

Nanocomposite active layer

Nanocomposite substrate

2 Fig 17: Schematic illustrations of different structures of TFC-N membranes: a) TFC-N with modified substrate, b) 3 TFC-N with modified active layer and c) TFC-N with modified both substrate and active layer 


\subsubsection{TFC-N with modified substrate}

\section{a) $\mathbf{T i O}_{2}$ nanoparticle in substrate}

3 Emadzadeh et al. [143] added titanium oxide $\left(\mathrm{TiO}_{2}\right)$ nanoparticles, in a range of 0-1 \%, to PSU

4 substrate which was coated with in-situ polymerized polyamide. By addition of the hydrophilic

$5 \mathrm{TiO}_{2}$ nanoparticles to the substrate forming a nano-composite sublayer, its hydrophilicity

6 increased. In Fig. 18, cross-sectional and surface SEM images are shown. From the figure, larger

7 finger-like pores were formed as well as the overall porosity increased upon addition of $\mathrm{TiO}_{2}$ due

8 to the increase in the water transfer rate from the coagulant to the polymer film. Further increase

9 in $\mathrm{TiO}_{2}$ concentration led to increase the nanoparticle agglomerates, observed as white spots. It

10 made the membrane surface rougher and the active layer more defective. As a result of these

11 morphological changes, water flux kept increasing while the salt rejection kept decreasing with an

12 increase in $\mathrm{TiO}_{2}$ addition. When the $\mathrm{TiO}_{2}$ concentration was higher than $0.5 \mathrm{wt} . \%$ reverse salt flux

13 became excessive due to the excessive nanoparticle agglomeration and defect formation. Hence, 14 it was concluded that $0.5 \mathrm{wt} . \%$ was the optimal value. 

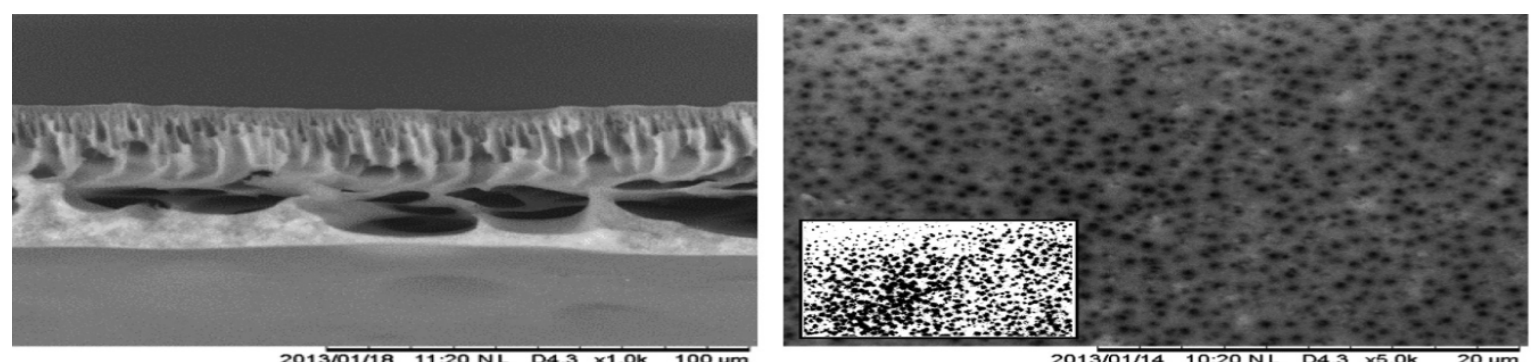

(a)
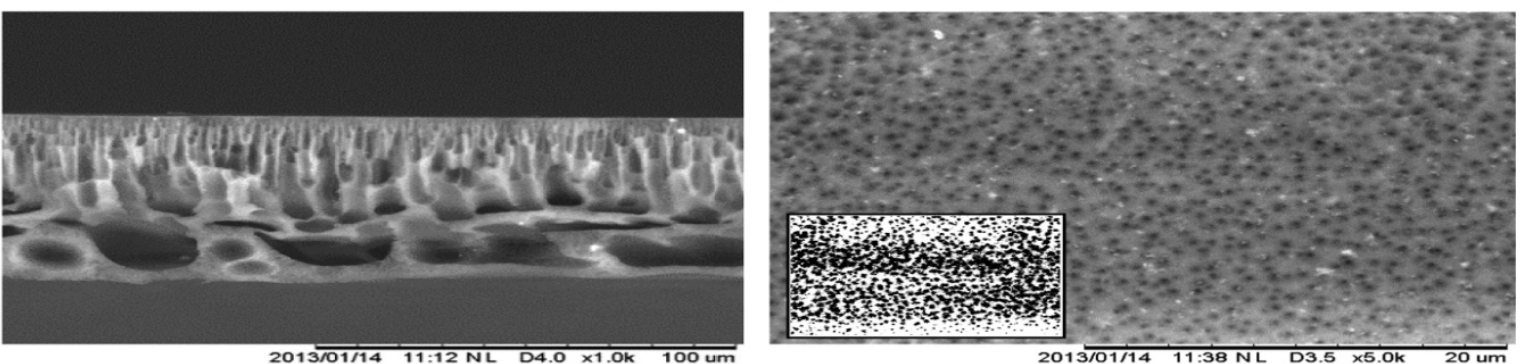

(b)
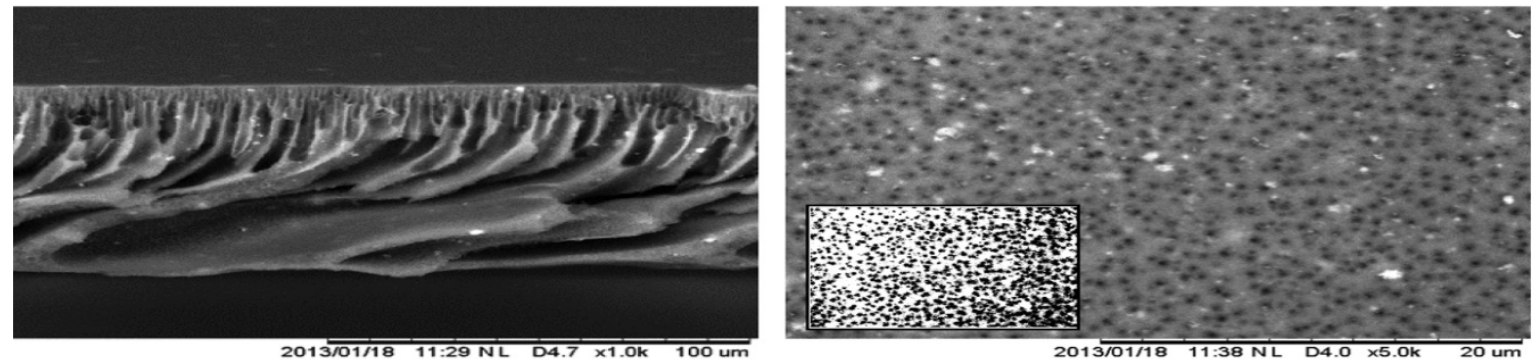

(C)
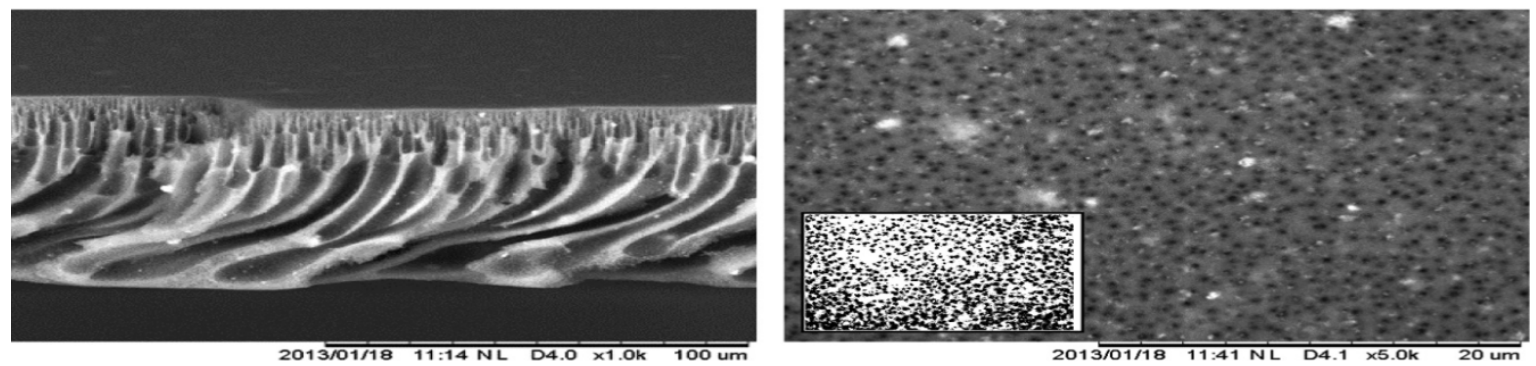

(d)

Fig 18: SEM of the cross-section and top surface of PSF substrates prepared from different nanoparticles loadings, (a) substrate (control), (b) substrate 0.5 , (c) substrate 0.75 and (d) substrate1.0 (note: threshold image placed on bottom left corner of each top surface was used to determine average pore size using ImageJ software) [143]

\section{b) ZnO nanoparticle in substrate}

Rastgar et al. [146] studied the effect of addition of two nanoparticles to PES substrate on the performance of a polyamide TFC membrane. The two applied nanoparticles were Zinc oxide nanoparticles (ZNPs) and $\mathrm{ZnO}^{-\mathrm{SiO}_{2}}$ core-shell nanoparticles (ZSCSNPs). ZNPs were prepared by

10 sol-gel method and then covered with hydrophilic silica $\left(\mathrm{SiO}_{2}\right)$ to synthesize ZSCSNPs which were 11 more hydrophilic than ZNPs. Table 4 shows the composition of the dope solution used to fabricate 
1 the membranes. The SEM images showed that the addition of nanoparticles to the substrate led to 2 the formation of a more finger-like structure and an increase in the total porosity. These changes 3 were due to the increase of water transfer from the coagulation to the polymeric film.

5 Table 4: The compositions of dope solution used to fabricate the substrates [146]

\begin{tabular}{lccccc}
\hline support layer label & $\begin{array}{c}\text { PES } \\
(\mathrm{wt} \%)\end{array}$ & $\begin{array}{c}\text { PEG-200 } \\
(\mathrm{wt} \%)\end{array}$ & $\begin{array}{c}\text { NMP } \\
(\mathrm{wt} \%)\end{array}$ & $\begin{array}{c}\text { Nanomaterial } \\
(\mathrm{type})\end{array}$ & Weight content \\
\hline PES & 18.00 & 10.00 & 72.00 & - & 0.00 \\
\hline PES-ZNP-1 & 18.00 & 10.00 & 72.00 & ZNPs & 1.00 \\
\hline PES-ZSCSNP-0.1 & 18.00 & 10.00 & 72.00 & ZSCSNPs & 0.10 \\
\hline PES-ZSCSNP-0.5 & 18.00 & 10.00 & 72.00 & ZSCSNPs & 0.50 \\
\hline PES-ZSCSNP-1 & 18.00 & 10.00 & 72.00 & ZSCSNPs & 1.00 \\
\hline PES-ZSCSNP-2 & 18.00 & 10.00 & 72.00 & ZSCSNPs & 2.00 \\
\hline
\end{tabular}

8 Fig. 19 shows the AFM surface images and the roughness parameters of the neat PES and the 9 nanocomposite substrates for their TFC-N membranes. As can be seen, the addition of the 10 nanoparticles increased the surface roughness, likely due to the nanoparticle agglomeration, Both 11 ZNP and ZSCSNP had the same effect but the effect of ZNP was stronger. Interestingly, the salt 12 rejection was almost the same after the addition of either of the two nanoparticles. It is presumably 13 due to the negligible difference in the size of the utilized nano-particles, i.e. 30 and $50 \mathrm{~nm}$. Despite 14 the higher roughness of ZNP added substrate, water flux of ZSCSNPs TFC-N membrane was 15 higher. It was most likely due to the higher hydrophilicity of ZSCSNP [146]. 

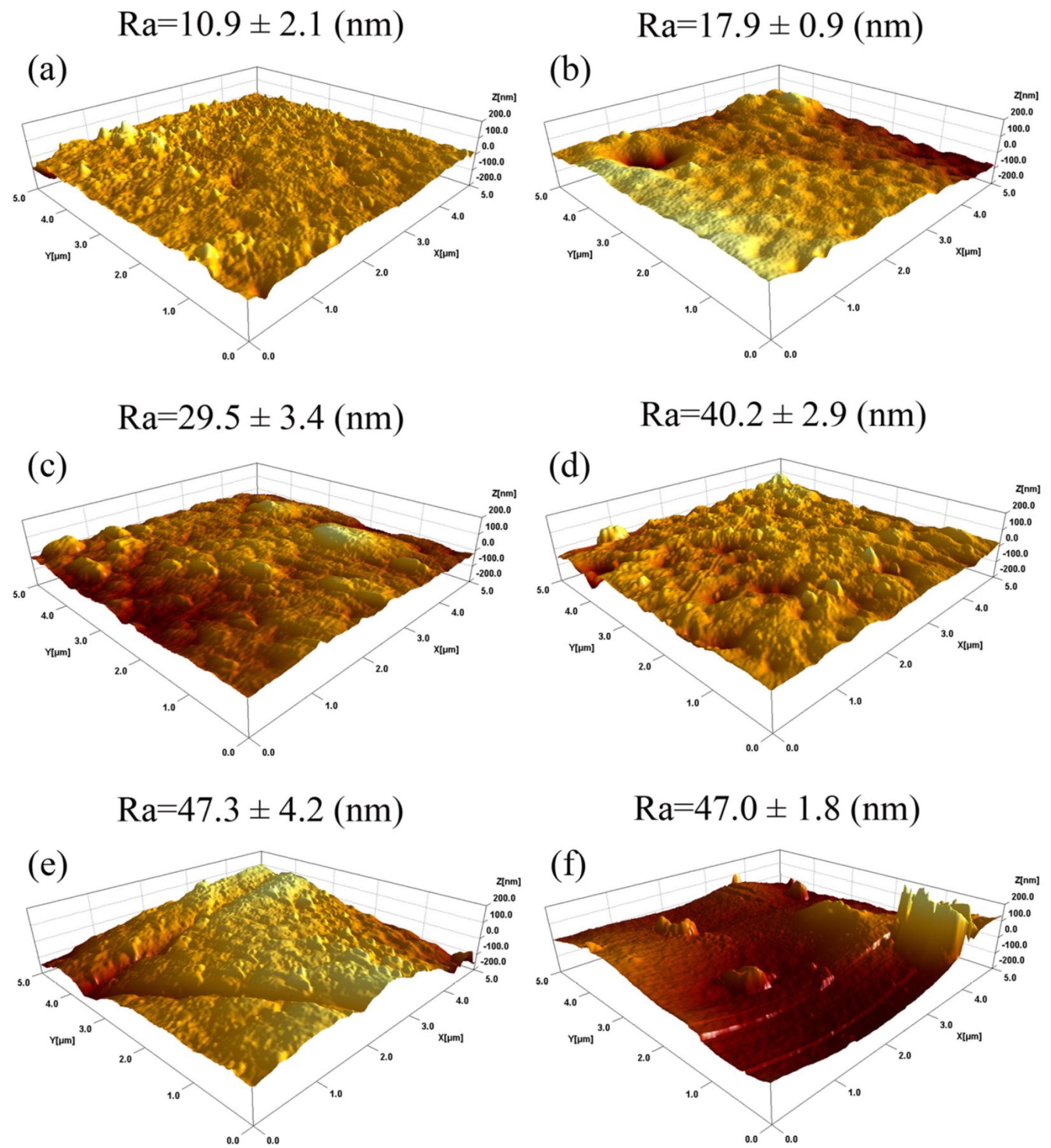

Fig 19: AFM images of PES-TFC-N (a), PES-ZSCSNP-0.1 (b), PES-ZSCSNP-0.5 (c), PES-ZSCSNP-1 (d), PESZSCSNP-2 (e) and PES-ZNP-1 (f) substrate layers. [146]

\section{c) Silica nanoparticle in substrate}

6 Tian et al. [147] incorporated silica nanoparticles in the TFC-N electro-spun polyetherimide (PEI) 7 nanofiber substrate to reduce the ICP in FO. In comparison with the finger-like structure formed 8 by the phase inversion method, the inter-connected macropores of nanofibrous membranes have 
1 the potential to decrease the membrane structural parameter, with lower tortuosity coefficient and

2 higher porosity. The reason for the addition of silica nanoparticles was to maintain the porosity 3 during the heat treatment of nanofibers either by heat-press. The outputs revealed high thermal 4 capability of the nanofibers synthesized based on silica nanoparticles during the heat-press 5 treatment.

Ma et al. [148] studied the effect of addition of zeolite nanoparticles into the polyamide active layer on the performance of TFC-N membranes in FO. PSF was used to make the substrate, and zeolite nanoparticles were added to the organic phase in interfacial polymerization. The surface morphology, roughness and contact angle all changed by the addition of zeolite nanoparticles. These changes finally affected the separation properties of the fabricated membranes. The addition of zeolite nanoparticles into the polyamide selective layer of FO membrane increased the FO

\section{b) Amino-functionalized titanate nanotubes in active layer}

Emadzadeh et al. [144] used Amino-functionalized titanate nanotubes $\left(\mathrm{NH}_{2}-\mathrm{TNTs}\right)$ in the organic phase to make TFC-N membrane for FO. Titanate nanotubes (TNTs) were surface modified by reacting with $\mathrm{N}$-(2-Aminoethyl)-3-aminopropyltrimethoxysilane (AAPTS) to form $\mathrm{NH}_{2}$-TNTs, which was confirmed by ATR-FTIR. Also, crystalline structure and tubular morphology of $\mathrm{NH}_{2}$ TNTs was characterized, by using XRD and TEM tests respectively. Nanoparticle $\mathrm{NH}_{2}$-TNTs bind chemically with the polyamide active layer. In Fig. 20, the bond between the polyamide and nanoparticle $\mathrm{NH}_{2}$-TNTs is shown. 


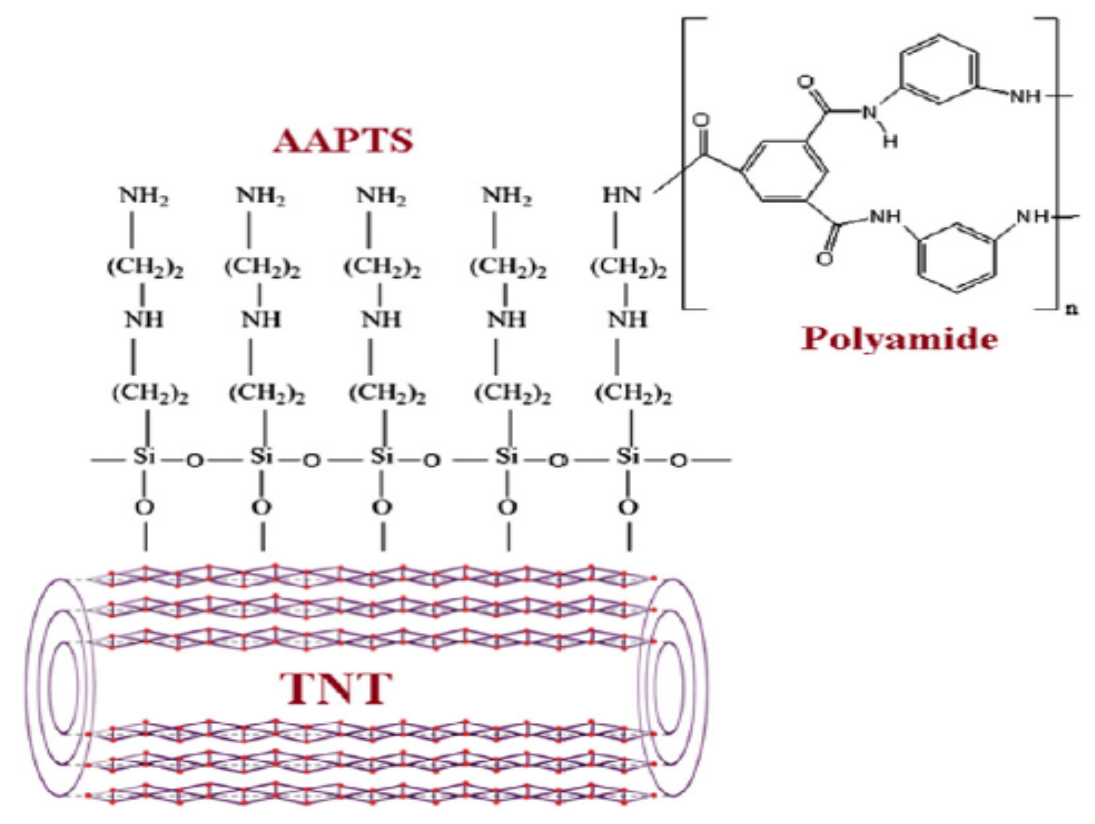

\section{Interaction between functionalized} TNTs and polyamide

Fig 20: Schematic illustration of interaction between polyamide (PA) and $\mathrm{NH}_{2}$-TNTs [144]

Fig. 21 shows the water flux and salt rejection as a function of $\mathrm{NH}_{2}$-TNTs content. Upon nanoparticle addition, water flux increased and it kept increasing with an increase in the nanoparticle concentration. The increase in water flux was caused by i) increase in hydrophilicity of the membrane, ii) increase in "leaf-like" and ridge and valley structure, iii) increase in the water current through the cavity between the nanoparticles and polymer matrix and iv) increase in the water current through the hollow nanoparticles. The increase of nanoparticle concentration up to $0.05 \mathrm{wt} . \%$ exhibited favorable effects such as increase in aqueous and organic phase compatibility and increase of the polyamide crosslinking via $-\mathrm{NH}_{2}$ groups attached to the nanoparticles in the active layer, resulted in higher salt rejection. However, further increase in nanoparticles concentration started to decrease the cross-linking and conversely increased the voids between the $\mathrm{NH}_{2}$-TNTs and polyamide matrix. As a result, salt rejection decreased [144]. This also was observed by the other researchers [149]. 


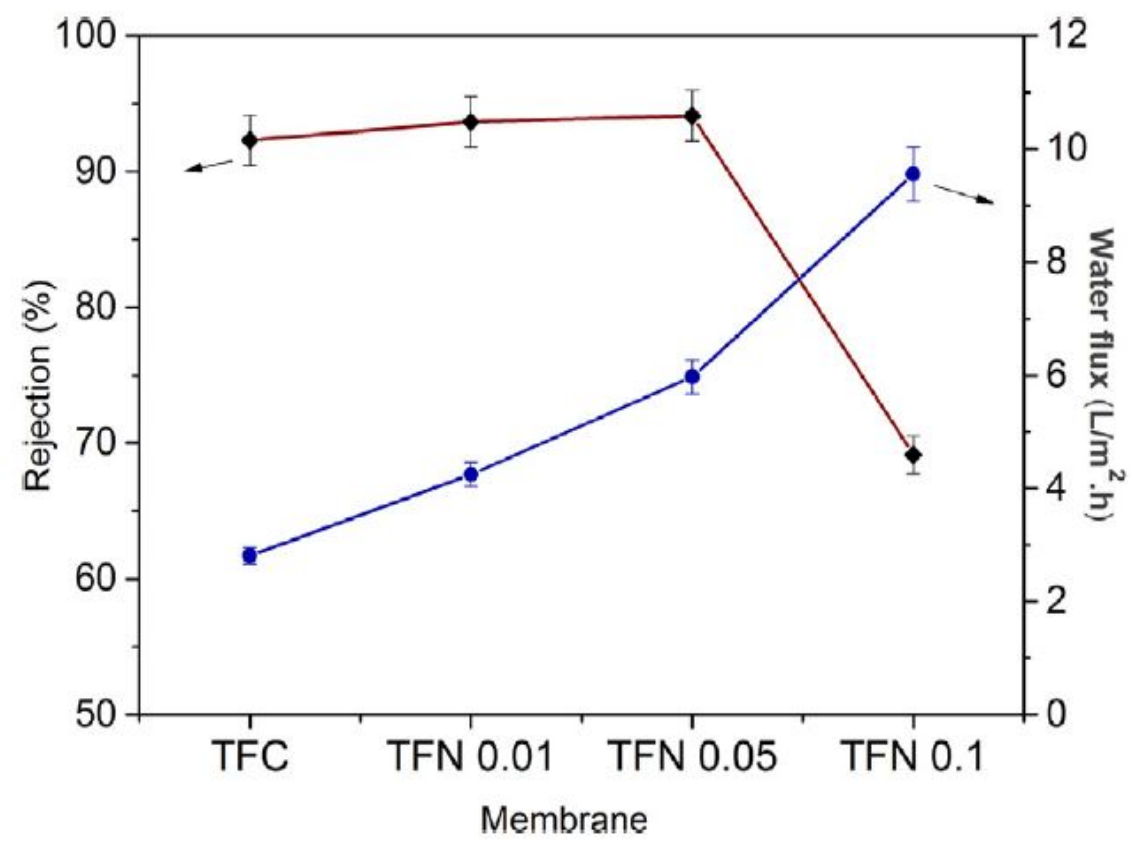

1

Fig 21: Water flux and $\mathrm{NaCl}$ rejection of TFC/TFC-N membranes (Test conditions: 2.5 bar, $25^{\circ} \mathrm{C}$ and $20 \mathrm{mM} \mathrm{NaCl}$ aqueous solution) [144]

\section{c) GOAg nanoparticles in active layer}

Faria et al. [139] used an antibacterial nanoparticle to make a TFC-N membrane. The nanoparticles (GOAg) were made of graphene oxide (GO) and silver nanoparticles (Ag). Also, a polyamide TFC membrane of HTI was used in this research. The carboxyl groups on the GOAg nanoparticle formed covalent bonds with the amine functional group on ethylene diamine (ED) functionalized TFC membrane. The nanoparticle showed antibacterial and antifouling effects on the membrane without changing its inherent properties. By addition of the nanoparticle, the flux reduction due to the membrane fouling decreased by $30 \%$.

\section{d) MOF in active layer}

Zirehpour et al.[150] synthesized nano-sized metal-organic framework (MOF) particles consisting of silver (I) and 1,3,5-benzene tricarboxylic acid. MOF was incorporated into the polyamide layer of membranes to improve the structure of TFC membrane. A good compatibility between the MOF and the aromatic polyamide layer was reported. This nano particle enhanced the hydrophilicity and transport properties of the active layer without any impact on selectivity. They also monitored the performance stability of the membrane in FO seawater desalination in the course of time. The Caspian seawater and $2 \mathrm{M} \mathrm{NaCl}$ was used as feed and draw solution respectively (Fig. 22). The FO seawater desalination flux through the TFC-N membrane was very stable throughout the testing interval (only about 7\% flux decline), while the normal TFC membrane presented about $18 \%$ reduction in water flux. The reductions in FO water flux are attributed mostly to the descent 
in driving force of FO process and membrane fouling. According to the vast variety of MOFs with different metal ions or clusters coordinated to different organic ligands to form one, two, or threedimensional structures, it is believed that the populated family of MOF needs more focus as an attractive research area in TFC-N membranes.

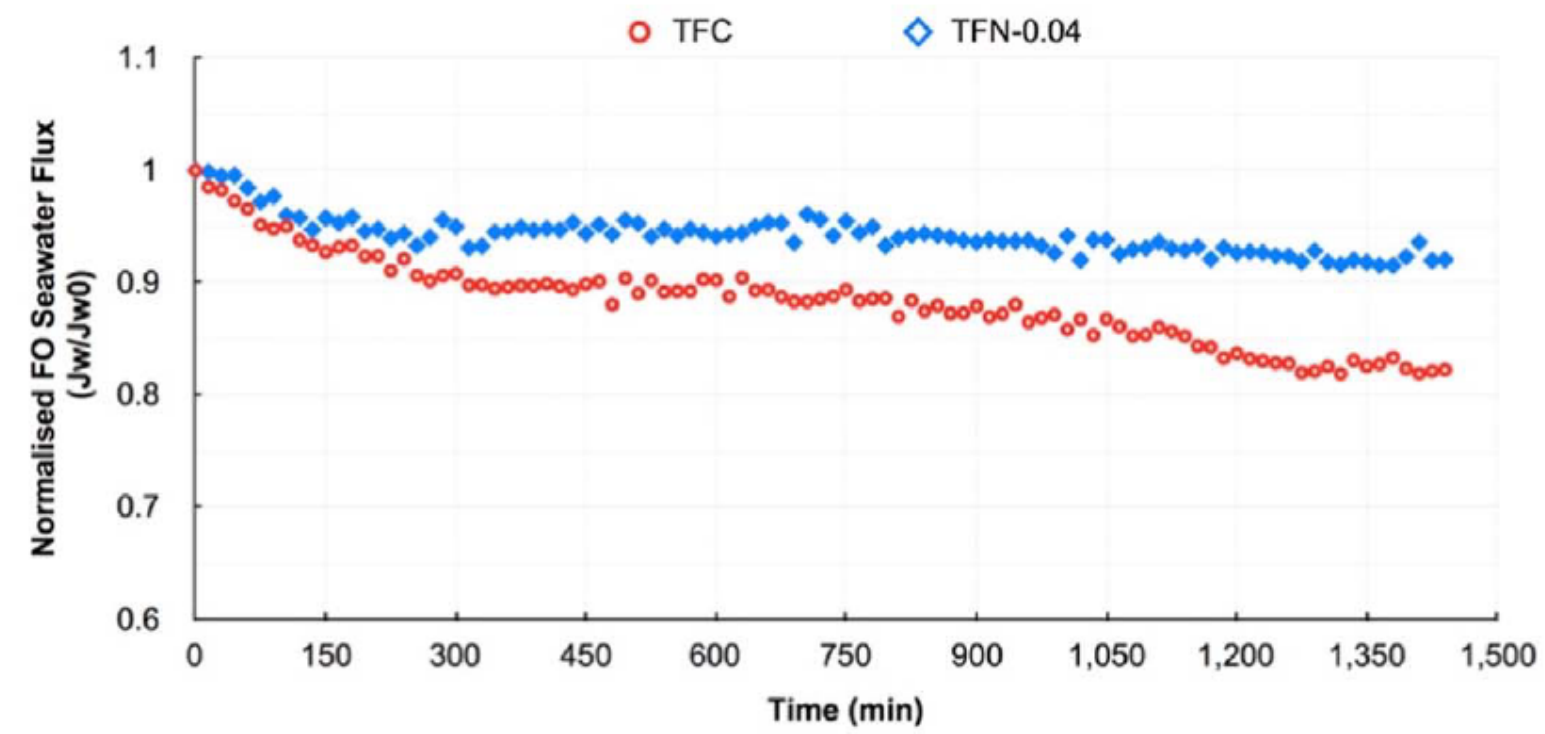

Fig 22: normalized (JW/Jw0) FO seawater flux decline of the membranes over time ( $\left.\mathrm{T}=25^{\circ} \mathrm{C}, \mathrm{AL}-\mathrm{FS}\right)[150]$

\subsubsection{TFC-N with modified both substrate and active layer concurrently}

Rezaei-DashtArzhandi et al. [149] fabricated TFC-N membranes with both substrate and PA active layer incorporated with Halloysite nanotubes (HNTs) and graphite-like carbon nitrite (g-C3N4), respectively. The contact angle measurement revealed that by adding g-C3N4 within the PA active layer super hydrophilic surface were formed. The FO water flux without increasing reverse solute flux was considerably enhanced by a combination of the positive features contributed by the substrate and the PA active layer modified by HNTs and g-C3N4 respectively. However, the results of this paper proved that the modification of the PA selective layer has presented dominant role towards improving FO membrane performances in comparison to the substrate modification. The antifouling property of TFC-N membranes can be attributed to the enhancement of surface hydrophilicity [151].

Also, Ohland et al.[152] used hydroxyapatite particles (Hapf) functionalized by plasma treatment as a nanoparticle additive into a porous cellulose acetate substrate and a selective polyamide layer concurrently. They concluded that the addition of the hydrophilic particles in porous CA substrate was able to enhance the hydrophilicity of the matrix and reduce ICP. Furthermore, Adding Hapf in the PA layer increased the affinity toward water and reorganized the polymer chains, resulting in lower diffusion resistance and enhancement of water permeability. 
1 Tables 5, 6 and 7 reviews the papers on the flux of water and the specific reverse salt flux of the 2 three types of TFC-N membranes respectively: TFC-N with modified substrate, TFC-N with 3 modified active layer and TFC-N with modified both substrate and active layer.

\section{Issues and implications of TFC-N membranes}

- Nanomaterials should be modified in order to become more compatible with substrate or active layer to prevent their loss during the process especially in the long term operation. It is suggested to examine the long-term operation of TFC-N membranes to evaluate the nanomaterial loss during the operation.

- Although the performance of FO membranes can be improved by applying a tiny amount of nanoparticles, nano-materials can be toxic and expensive. Therefore, nontoxic and costeffective nanomaterials should be used in an FO water treatment process. 
1 Table 5: A summary of the studies on TFC-N with modified substrate

\begin{tabular}{|c|c|c|c|c|c|c|c|c|c|c|c|}
\hline \multirow{2}{*}{\multicolumn{2}{|c|}{$\begin{array}{cc}\begin{array}{c}\text { Support } \\
\text { layer }\end{array} \\
\text { Active layer } \\
\end{array}$}} & \multirow[b]{3}{*}{ Structure } & \multicolumn{4}{|c|}{ Performance } & \multicolumn{3}{|c|}{ Experimental condition } & \multirow[b]{3}{*}{$\begin{array}{c}\text { Intrinsic } \\
\text { properties }\end{array}$} & \multirow[b]{3}{*}{ ref } \\
\hline & & & \multicolumn{2}{|c|}{ AL-FS(FO) } & \multicolumn{2}{|c|}{ AL-DS(PRO) } & \multirow[b]{2}{*}{$\begin{array}{c}\text { Feed } \\
\text { solution }\end{array}$} & \multirow[b]{2}{*}{$\begin{array}{r}\text { Draw } \\
\text { solution }\end{array}$} & \multirow[b]{2}{*}{$\begin{array}{c}\text { cross-flow } \\
\text { velocity }\end{array}$} & & \\
\hline & Nano material & & $\begin{array}{c}\mathrm{J}_{\mathrm{v}} \\
(\mathrm{LMH})\end{array}$ & $\begin{array}{l}\mathrm{J}_{\mathrm{S}} / \mathrm{J}_{\mathrm{v}} \\
(\mathrm{g} / \mathrm{L})\end{array}$ & $\begin{array}{c}\mathrm{J}_{\mathrm{v}} \\
(\mathrm{LMH})\end{array}$ & $\begin{array}{l}\mathrm{J}_{\mathrm{S}} / \mathrm{J}_{\mathrm{v}} \\
(\mathrm{g} / \mathrm{L})\end{array}$ & & & & & \\
\hline & $\begin{array}{c}\text { PSF } \\
\text { PA } \\
\text { Zeolite }\end{array}$ & Flat sheet & 40 & 0.7 & 86 & 0.66 & DI water & $\begin{array}{c}2 \mathrm{M} \\
\mathrm{NaCl}\end{array}$ & \multicolumn{3}{|c|}{$\begin{array}{c}\mathrm{A}=3.2 \mathrm{LMH} / \mathrm{bar} \\
\mathrm{S}=0.34 \mathrm{~mm} \\
\mathrm{R}=90.6\end{array}$} \\
\hline & $\begin{array}{l}\mathrm{PSF} \\
\mathrm{PA} \\
\mathrm{TiO}_{2}\end{array}$ & Flat sheet & 29.76 & 0.26 & 56.27 & 0.25 & $\begin{array}{c}10 \mathrm{mM} \\
\mathrm{NaCl}\end{array}$ & $\begin{array}{l}2 \mathrm{M} \\
\mathrm{NaCl}\end{array}$ & $\begin{array}{l}\mathrm{A}=1 \\
\mathrm{~B}=1\end{array}$ & $\begin{array}{l}=1.96 \mathrm{LMH} / \mathrm{bar} \\
=10.66 \times 10^{-8} \mathrm{~m} / \mathrm{s} \\
\mathrm{S}=.42 \mathrm{~mm} \\
\mathrm{R}=92.4\end{array}$ & [143] \\
\hline & $\begin{array}{l}\text { PSF } \\
\text { PA } \\
\text { GO }\end{array}$ & Flat sheet & 19.77 & 0.16 & 40.5 & 0.16 & DI water & $\begin{array}{r}0.5 \mathrm{M} \\
\mathrm{NaCl}\end{array}$ & $\begin{array}{r}\mathrm{A}=1 \\
\mathrm{~B}=\end{array}$ & $\begin{array}{c}=1.76 \mathrm{LMH} / \mathrm{bar} \\
\mathrm{B}=0.19 \mathrm{LMH} \\
\mathrm{S}=191 \mu \mathrm{m} \\
\mathrm{R}=98.71\end{array}$ & {$[154]$} \\
\hline & $\begin{array}{l}\text { PSF } \\
\text { PA } \\
\text { silica }\end{array}$ & Flat sheet & 31 & 0.24 & 60.5 & 0.26 & DI water & $\begin{array}{l}1 \mathrm{M} \\
\mathrm{NaCl}\end{array}$ & $\begin{array}{r}\mathrm{A}=1 \\
\mathrm{~B}=\end{array}$ & $\begin{array}{l}=1.64 \mathrm{LMH} / \mathrm{bar} \\
\mathrm{B}=0.29 \mathrm{LMH} \\
\mathrm{S}=169 \mu \mathrm{m}\end{array}$ & {$[155]$} \\
\hline & $\begin{array}{c}\text { polyetherimide } \\
\text { (PEI) } \\
\text { PA } \\
\text { carbon nanotubes } \\
\text { (CNTs) }\end{array}$ & Flat sheet & 32.8 & 0.12 & 61.3 & 0.07 & DI water & $\begin{array}{l}1 \mathrm{M} \\
\mathrm{NaCl}\end{array}$ & $\begin{array}{r}\mathrm{A}= \\
\mathrm{B}\end{array}$ & $\begin{array}{l}=2.6 \mathrm{LMH} / \mathrm{bar} \\
\mathrm{B}=0.7 \mathrm{LMH} \\
\mathrm{S}=210 \mu \mathrm{m}\end{array}$ & {$[156]$} \\
\hline $\begin{array}{l}\text { PSF } \\
\text { PA } \\
\text { HNTs }\end{array}$ & Flat sheet & 27.71 & 0.52 & 43.25 & 0.63 & $\begin{array}{c}10 \mathrm{mM} \\
\mathrm{NaCl}\end{array}$ & $\begin{array}{l}2 \mathrm{M} \\
\mathrm{NaCl}\end{array}$ & $350 \mathrm{ml} / \mathrm{min}$ & $\begin{array}{c}\mathrm{A}=2 \mathrm{LMH} / \mathrm{bar} \\
\mathrm{B}=9.43 \times 10^{-8} \mathrm{~m} / \mathrm{s} \\
\mathrm{S}=0.37 \mathrm{~mm}\end{array}$ & [157] & \\
\hline $\begin{array}{r}\text { PSF } \\
\text { PA } \\
\text { Layered doub } \\
\text { hydroxide (LI }\end{array}$ & Flat sheet & 18.1 & 0.44 & 34.6 & 0.36 & DI water & $\begin{array}{c}1 \mathrm{M} \\
\mathrm{NaCl}\end{array}$ & $190 \mathrm{ml} / \mathrm{min}$ & $\mathrm{S}=57.4 \mu \mathrm{m}$ & {$[158]$} & \\
\hline
\end{tabular}

2 
2 Table 5 (continued)

\begin{tabular}{|c|c|c|c|c|c|c|c|c|c|c|}
\hline \multirow{2}{*}{$\begin{array}{c}\begin{array}{c}\text { Support } \\
\text { layer }\end{array} \\
\text { Active layer } \\
\end{array}$} & \multirow[b]{3}{*}{ structure } & \multicolumn{4}{|c|}{ performance } & \multicolumn{3}{|c|}{ Experimental condition } & \multirow[b]{3}{*}{$\begin{array}{c}\text { Intrinsic } \\
\text { properties }\end{array}$} & \multirow[b]{3}{*}{ ref } \\
\hline & & \multicolumn{2}{|c|}{ AL-FS(FO) } & \multicolumn{2}{|c|}{ AL-DS(PRO) } & \multirow[b]{2}{*}{$\begin{array}{c}\text { Feed } \\
\text { solution }\end{array}$} & \multirow[b]{2}{*}{$\begin{array}{r}\text { Draw } \\
\text { solution }\end{array}$} & \multirow[b]{2}{*}{$\begin{array}{c}\text { cross-flow } \\
\text { velocity }\end{array}$} & & \\
\hline Nano material & & $\begin{array}{c}\mathrm{J}_{\mathrm{v}} \\
(\mathrm{LMH})\end{array}$ & $\begin{array}{l}J_{\mathrm{S}} / \mathrm{J}_{\mathrm{v}} \\
(\mathrm{g} / \mathrm{L})\end{array}$ & $\begin{array}{c}\mathrm{J}_{\mathrm{v}} \\
(\mathrm{LMH})\end{array}$ & $\begin{array}{l}\mathrm{J}_{\mathrm{s}} / \mathrm{J}_{\mathrm{v}} \\
(\mathrm{g} / \mathrm{L})\end{array}$ & & & & & \\
\hline $\begin{array}{c}\text { PSF } \\
\text { PA } \\
\text { LDH/GO }\end{array}$ & Flat sheet & 13.4 & 0.46 & 23.6 & 0.29 & DI water & $\begin{array}{c}1 \mathrm{M} \\
\mathrm{NaCl}\end{array}$ & $190 \mathrm{ml} / \mathrm{min}$ & $\begin{array}{c}\mathrm{A}=0.53 \mathrm{LMH} / \mathrm{bar} \\
\mathrm{B}=0.15 \mathrm{LMH} \\
\mathrm{S}=138 \mu \mathrm{m}\end{array}$ & {$[159]$} \\
\hline $\begin{array}{c}\text { PES } \\
\mathrm{PA} \\
\mathrm{ZnO}\end{array}$ & Flat sheet & 31 & 0.41 & 43 & 0.42 & DI water & $\begin{array}{c}1 \mathrm{M} \\
\mathrm{NaCl}\end{array}$ & $8.3 \mathrm{~cm} / \mathrm{s}$ & $\begin{array}{c}\mathrm{A}=3.12 \mathrm{LMH} / \mathrm{bar} \\
\mathrm{B}=3.75 \mathrm{LMH} \\
\mathrm{S}=300 \mu \mathrm{m} \\
\mathrm{R}=77.94\end{array}$ & {$[146]$} \\
\hline $\begin{array}{c}\text { PES } \\
\text { PA } \\
\text { ZSCSNPs }\end{array}$ & Flat sheet & 33.5 & 0.36 & 50.1 & 0.36 & DI water & $\begin{array}{c}1 \mathrm{M} \\
\mathrm{NaCl}\end{array}$ & $8.3 \mathrm{~cm} / \mathrm{s}$ & $\begin{array}{c}\mathrm{A}=3.47 \mathrm{LMH} / \mathrm{bar} \\
\mathrm{B}=4.01 \mathrm{LMH} \\
\mathrm{S}=297 \mu \mathrm{m} \\
\mathrm{R}=78.60\end{array}$ & {$[146]$} \\
\hline $\begin{array}{c}\mathrm{PEI} \\
\mathrm{PA} \\
\mathrm{SiO}_{2}\end{array}$ & Flat sheet & 42 & 0.12 & 72 & 0.1 & DI water & $\begin{array}{l}1 \mathrm{M} \\
\mathrm{NaCl}\end{array}$ & $9 \mathrm{~cm} / \mathrm{s}$ & $\begin{array}{c}\mathrm{A}=2.99 \mathrm{LMH} / \mathrm{bar} \\
\mathrm{B}=0.41 \mathrm{LMH} \\
\mathrm{S}=174 \mu \mathrm{m} \\
\mathrm{R}=74.2\end{array}$ & {$[147]$} \\
\hline $\begin{array}{l}\text { PSF } \\
\text { PA } \\
\text { GO }\end{array}$ & $\begin{array}{c}\text { Dual- } \\
\text { layered } \\
\text { Flat sheet }\end{array}$ & 33.8 & 0.19 & 61.5 & 0.18 & DI water & $\begin{array}{c}1 \mathrm{M} \\
\mathrm{NaCl}\end{array}$ & $16.7 \mathrm{~cm} / \mathrm{s}$ & $\begin{array}{c}\mathrm{A}=1.46 \mathrm{LMH} / \mathrm{bar} \\
\mathrm{B}=0.25 \mathrm{LMH} \\
\mathrm{S}=130 \mu \mathrm{m}\end{array}$ & {$[160]$} \\
\hline $\begin{array}{c}\text { PSF } \\
\text { PA } \\
\text { Imogolite } \\
\text { nanotubes (INTs) }\end{array}$ & Flat sheet & 7.17 & 1.47 & 9.5 & 2.6 & DI water & $\begin{array}{l}1 \mathrm{M} \\
\mathrm{NaCl}\end{array}$ & $\begin{array}{c}333.3 \\
\mathrm{ml} / \mathrm{min}\end{array}$ & $\begin{array}{c}\mathrm{A}=3.03 \mathrm{LMH} / \mathrm{bar} \\
\mathrm{B}=2.92 \mathrm{LMH} \\
\mathrm{S}=2.09 \mathrm{~mm} \\
\mathrm{R}=83\end{array}$ & {$[161]$} \\
\hline $\begin{array}{c}\text { PSF } \\
\text { PA } \\
\text { MOF (UiO-66) }\end{array}$ & Flat sheet & 24.5 & 0.18 & 39.4 & & DI water & $\begin{array}{l}1 \mathrm{M} \\
\mathrm{NaCl}\end{array}$ & $1.1 \mathrm{~cm} / \mathrm{s}$ & $\begin{array}{c}\mathrm{A}=3.31 \mathrm{LMH} / \mathrm{bar} \\
\mathrm{B}=0.53 \mathrm{LMH} \\
\mathrm{S}=351 \mu \mathrm{m} \\
\mathrm{R}=92.6\end{array}$ & {$[162]$} \\
\hline
\end{tabular}


1 Table 6: A summary of the studies on TFC-N with modified active layer

\begin{tabular}{|c|c|c|c|c|c|c|c|c|c|c|}
\hline \multirow{2}{*}{$\begin{array}{c}\begin{array}{c}\text { Support } \\
\text { layer }\end{array} \\
\text { Active layer }\end{array}$} & \multirow[b]{3}{*}{ structure } & \multicolumn{4}{|c|}{ performance } & \multicolumn{3}{|c|}{ Experimental condition } & \multirow[b]{3}{*}{$\begin{array}{l}\text { Intrinsic } \\
\text { properties }\end{array}$} & \multirow[b]{3}{*}{ ref } \\
\hline & & \multicolumn{2}{|c|}{ AL-FS(FO) } & \multicolumn{2}{|c|}{ AL-DS(PRO) } & \multirow[b]{2}{*}{$\begin{array}{c}\text { Feed } \\
\text { solution }\end{array}$} & \multirow[b]{2}{*}{$\begin{array}{r}\text { Draw } \\
\text { solution }\end{array}$} & \multirow[b]{2}{*}{$\begin{array}{l}\text { cross-flow } \\
\text { velocity }\end{array}$} & & \\
\hline Nano material & & $\begin{array}{c}\mathrm{J}_{\mathrm{v}} \\
(\mathrm{LMH})\end{array}$ & $\begin{array}{l}\mathrm{J}_{\mathrm{s}} / \mathrm{J}_{\mathrm{v}} \\
(\mathrm{g} / \mathrm{L})\end{array}$ & $\begin{array}{c}\mathrm{J}_{\mathrm{v}} \\
(\mathrm{LMH})\end{array}$ & $\begin{array}{l}\mathrm{J}_{\mathrm{s}} / \mathrm{J}_{\mathrm{v}} \\
(\mathrm{g} / \mathrm{L})\end{array}$ & & & & & \\
\hline PSF & & & & & & & & & $\mathrm{A}=7.15 \times 10^{-12} \mathrm{~m} / \mathrm{s} \mathrm{pa}$ & \\
\hline $\begin{array}{c}\text { PA } \\
\text { Zeolite }\end{array}$ & Flat sheet & 17.4 & 0.45 & 38.2 & 0.37 & DI water & $1 \mathrm{M} \mathrm{NaCl}$ & $500 \mathrm{~mL} / \mathrm{min}$ & $\begin{array}{c}\mathrm{B}=43.7 \times 10^{-8} \mathrm{~m} / \mathrm{s} \\
\mathrm{R}=77.6\end{array}$ & [148] \\
\hline $\begin{array}{c}\mathrm{PSF} \\
\mathrm{PA} \\
\mathrm{SiO} 2\end{array}$ & Flat sheet & 15 & 0.1 & 25 & 0.14 & $\begin{array}{c}10 \mathrm{mM} \\
\mathrm{NaCl}\end{array}$ & $2 \mathrm{M} \mathrm{NaCl}$ & $800 \mathrm{~mL} / \mathrm{min}$ & $\begin{array}{c}\mathrm{A}=9.52 \times 10^{-12} \mathrm{~m} / \mathrm{s} \mathrm{pa} \\
\mathrm{B}=28.4 \times 10^{-8} \mathrm{~m} / \mathrm{s} \\
\mathrm{S}=0.368 \mathrm{~mm} \\
\mathrm{R}=89\end{array}$ & [138] \\
\hline $\begin{array}{c}\mathrm{PSF} \\
\mathrm{PA} \\
\mathrm{NH}_{2}-\mathrm{TNTs} \\
\end{array}$ & Flat sheet & 17.82 & 0.12 & 32 & 0.14 & $\begin{array}{c}10 \mathrm{mM} \\
\mathrm{NaCl}\end{array}$ & $1 \mathrm{M} \mathrm{NaCl}$ & $32.72 \mathrm{~cm} / \mathrm{s}$ & $\begin{array}{c}\mathrm{A}=2.39 \mathrm{LMH} / \mathrm{bar} \\
\mathrm{B}=10.30 \times 10^{-8} \mathrm{~m} / \mathrm{s} \\
\mathrm{R}=94.1\end{array}$ & [144] \\
\hline $\begin{array}{l}\text { PAN } \\
\text { PA } \\
\text { GO }\end{array}$ & Flat sheet & 31.7 & 0.21 & 47 & 0.24 & DI water & $2 \mathrm{M} \mathrm{NaCl}$ & $300 \mathrm{ml} / \mathrm{min}$ & $\begin{array}{c}\mathrm{A}=2.04 \mathrm{LMH} / \mathrm{bar} \\
\mathrm{B}=0.83 \mathrm{LMH} \\
\mathrm{S}=0.085 \mathrm{~mm} \\
\mathrm{R}=86.43\end{array}$ & [163] \\
\hline $\begin{array}{c}\mathrm{PSF} \\
\mathrm{PA} \\
\mathrm{TiO}_{2} \\
\end{array}$ & Flat sheet & 26 & 0.19 & 34.4 & 0.18 & $\begin{array}{c}10 \mathrm{mM} \\
\mathrm{NaCl}\end{array}$ & $\begin{array}{r}0.5 \mathrm{M} \\
\mathrm{NaCl}\end{array}$ & $300 \mathrm{~mL} / \mathrm{min}$ & & [164] \\
\hline $\begin{array}{c}\text { PSF } \\
\text { PA } \\
\text { MOF ( UiO-66) }\end{array}$ & Flat sheet & 27 & 0.23 & 51.3 & 0.24 & DI water & $2 \mathrm{M} \mathrm{NaCl}$ & $1.1 \mathrm{~cm} / \mathrm{s}$ & $\begin{array}{c}\mathrm{A}=3.3 \mathrm{LMH} / \mathrm{bar} \\
\mathrm{B}=0.3 \mathrm{LMH} \\
\mathrm{S}=1637 \mu \mathrm{m} \\
\mathrm{R}=95.3\end{array}$ & [165] \\
\hline $\begin{array}{c}\text { PES } \\
\text { PA } \\
\text { MOF ( UiO-66) }\end{array}$ & Flat sheet & 47 & 0.14 & & & DI water & $2 \mathrm{M} \mathrm{NaCl}$ & $21 \mathrm{~cm} / \mathrm{s}$ & $\begin{array}{c}\mathrm{A}=4.8 \mathrm{LMH} / \mathrm{bar} \\
\mathrm{B}=0.6 \mathrm{LMH}\end{array}$ & [150] \\
\hline $\begin{array}{c}\text { PSF } \\
\text { PA } \\
\text { GO }\end{array}$ & Flat sheet & 14.5 & 0.17 & 34.7 & 0.13 & DI water & $1 \mathrm{M} \mathrm{NaCl}$ & $25000 \mathrm{ml} / \mathrm{min}$ & $\begin{array}{c}\mathrm{A}=6.52 \times 10^{-12} \mathrm{~m} / \mathrm{s} \mathrm{pa} \\
\mathrm{B}=18.7 \times 10^{-8} \mathrm{~m} / \mathrm{s} \\
\mathrm{R}=88\end{array}$ & [166] \\
\hline
\end{tabular}


Table 6(continued)

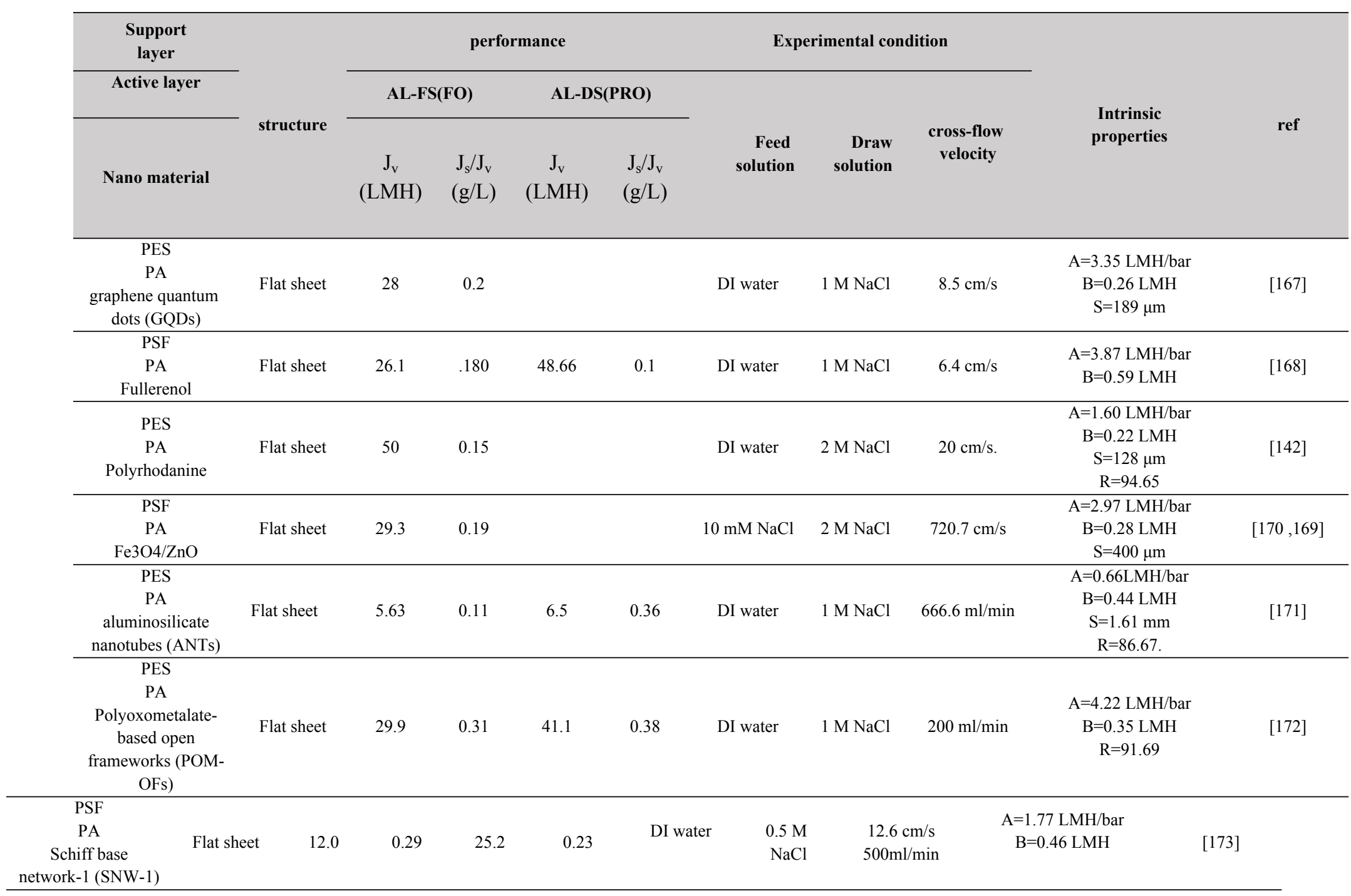


3 Table 6(continued)

\begin{tabular}{|c|c|c|c|c|c|c|c|c|c|c|}
\hline Support & \multirow[b]{3}{*}{ structure } & \multicolumn{4}{|c|}{ performance } & \multicolumn{3}{|c|}{ Experimental condition } & \multirow[b]{3}{*}{$\begin{array}{c}\text { Intrinsic } \\
\text { properties }\end{array}$} & \multirow[b]{3}{*}{ ref } \\
\hline Active layer & & \multicolumn{2}{|c|}{ AL-FS(FO) } & \multicolumn{2}{|c|}{ AL-DS(PRO) } & \multirow[b]{2}{*}{$\begin{array}{l}\text { Feed } \\
\text { solution }\end{array}$} & \multirow[b]{2}{*}{$\begin{array}{r}\text { Draw } \\
\text { solution }\end{array}$} & \multirow[b]{2}{*}{$\begin{array}{c}\text { cross-flow } \\
\text { velocity }\end{array}$} & & \\
\hline Nano material & & $\begin{array}{r}\mathrm{J}_{\mathrm{v}} \\
(\mathrm{LMH})\end{array}$ & $\begin{array}{r}\mathrm{J}_{\mathrm{S}} / \mathrm{J}_{\mathrm{V}} \\
(\mathrm{g} / \mathrm{L})\end{array}$ & $\begin{array}{r}\mathrm{J}_{\mathrm{v}} \\
(\mathrm{LMH})\end{array}$ & $\begin{array}{l}\mathrm{J}_{\mathrm{s}} / \mathrm{J}_{\mathrm{v}} \\
(\mathrm{g} / \mathrm{L})\end{array}$ & & & & & \\
\hline $\begin{array}{c}\text { PES } \\
\text { PA } \\
\text { MOF } \\
\text { (copper 1,4- } \\
\text { benzenedicarboxylate } \\
\text { nanosheets, CuBDC- } \\
\text { NS) }\end{array}$ & Flat sheet & 27.4 & 0.11 & 49.3 & 0.11 & DI water & $\begin{array}{l}1 \mathrm{M} \\
\mathrm{NaCl}\end{array}$ & $15 \mathrm{~cm} / \mathrm{s}$ & $\begin{array}{c}\mathrm{A}=3.13 \mathrm{LMH} / \mathrm{bar} \\
\mathrm{B}=0.317 \mathrm{LMH} \\
\mathrm{S}=366\end{array}$ & {$[174]$} \\
\hline $\begin{array}{c}\text { PES } \\
\text { PA } \\
\text { polyoxometalate } \\
\text { based open } \\
\text { frameworks (POM- } \\
\text { OFs) } \\
\end{array}$ & Flat sheet & 26.7 & 0.32 & 46.4 & 0.32 & DI water & $\begin{array}{l}1 \mathrm{M} \\
\mathrm{NaCl}\end{array}$ & $200 \mathrm{ml} / \mathrm{min}$ & & {$[175]$} \\
\hline $\begin{array}{c}\text { PES } \\
\text { PA } \\
\text { mesoporous silica } \\
\text { modified with amine } \\
\text { (SBA-15-NH2) }\end{array}$ & Flat sheet & 55.3 & 0.3 & 80.4 & 0.28 & DI water & $\begin{array}{l}1 \mathrm{M} \\
\mathrm{NaCl}\end{array}$ & $200 \mathrm{ml} / \mathrm{min}$ & & {$[176]$} \\
\hline $\begin{array}{c}\text { PES } \\
\text { PA } \\
\text { GO-oxidized carbon } \\
\text { nanotubes (OCNTs) } \\
\end{array}$ & Flat sheet & 84.6 & 0.04 & 114 & 0.04 & DI water & $\begin{array}{l}1 \mathrm{M} \\
\mathrm{NaCl}\end{array}$ & $\begin{array}{l}1500 \\
\mathrm{ml} / \mathrm{min}\end{array}$ & & {$[177]$} \\
\hline $\begin{array}{c}\text { PES } \\
\text { PA } \\
\text { GQDs@UiO-66- } \\
\text { NH2 }\end{array}$ & Flat sheet & 59.3 & 0.32 & 85.3 & 0.42 & DI water & $\begin{array}{l}1 \mathrm{M} \\
\mathrm{NaCl}\end{array}$ & $200 \mathrm{ml} / \mathrm{min}$ & $\begin{array}{c}\mathrm{A}=4.88 \mathrm{LMH} / \mathrm{bar} \\
\mathrm{B}=1.356 \mathrm{LMH} \\
\mathrm{S}=366\end{array}$ & {$[178]$} \\
\hline
\end{tabular}


2 Table 7: A summary of the studies on TFC-N with modified both substrate and active layer

\begin{tabular}{|c|c|c|c|c|c|c|c|c|c|c|}
\hline \multirow{2}{*}{$\begin{array}{c}\begin{array}{c}\text { Support } \\
\text { layer }\end{array} \\
\text { Active layer }\end{array}$} & \multirow[b]{3}{*}{ structure } & \multicolumn{4}{|c|}{ performance } & \multicolumn{3}{|c|}{ Experimental condition } & \multirow[b]{3}{*}{$\begin{array}{c}\text { Intrinsic } \\
\text { properties }\end{array}$} & \multirow[b]{3}{*}{ ref } \\
\hline & & \multicolumn{2}{|c|}{ AL-FS(FO) } & \multicolumn{2}{|c|}{ AL-DS(PRO) } & \multirow[b]{2}{*}{$\begin{array}{c}\text { Feed } \\
\text { solution }\end{array}$} & \multirow[b]{2}{*}{$\begin{array}{c}\text { Draw } \\
\text { solution }\end{array}$} & \multirow[b]{2}{*}{$\begin{array}{c}\text { cross-flow } \\
\text { velocity }\end{array}$} & & \\
\hline Nano material & & $\begin{array}{c}\mathrm{J}_{\mathrm{v}} \\
(\mathrm{LMH})\end{array}$ & $\begin{array}{c}\mathrm{J}_{\mathrm{s}} / \mathrm{J}_{\mathrm{v}} \\
(\mathrm{g} / \mathrm{L})\end{array}$ & $\begin{array}{c}\mathrm{J}_{\mathrm{v}} \\
(\mathrm{LMH})\end{array}$ & $\begin{array}{l}\mathrm{J}_{\mathrm{s}} / \mathrm{J}_{\mathrm{V}} \\
(\mathrm{g} / \mathrm{L})\end{array}$ & & & & & \\
\hline PSF & & & & & & & & & $\mathrm{A}=8.43 \mathrm{LMH} / \mathrm{bar}$ & \\
\hline $\begin{array}{c}\text { PA } \\
\text { aluminum oxide }\left(\mathrm{Al}_{2} \mathrm{O}_{3}\right)\end{array}$ & Flat sheet & 27.6 & 0.25 & 51.5 & 0.05 & DI water & $1 \mathrm{M} \mathrm{NaCl}$ & $18.5 \mathrm{~cm} / \mathrm{s}$ & $\begin{array}{c}\mathrm{B}=1.66 \mathrm{LMH} \\
\mathrm{S}=1028 \mu \mathrm{m}\end{array}$ & [179] \\
\hline $\begin{array}{c}\text { PSF } \\
\text { PA } \\
\text { HNTs } \\
\text { g-C3N4 }\end{array}$ & Flat sheet & 18.88 & 0.14 & & & DI water & $2 \mathrm{M} \mathrm{NaCl}$ & $21.4 \mathrm{~cm} / \mathrm{s}$ & $\begin{array}{c}\mathrm{A}=2.171 \mathrm{LMH} / \mathrm{bar} \\
\mathrm{B}=10.5 \times 10^{-8} \mathrm{~m} / \mathrm{s} \\
\mathrm{S}=0.37 \mathrm{~mm} \\
\mathrm{R}=93\end{array}$ & [149] \\
\hline $\begin{array}{c}\text { CA } \\
\text { PA } \\
\text { Hapf }\end{array}$ & Flat sheet & & & 22.6 & 0.6 & DI water & $1 \mathrm{M} \mathrm{NaCl}$ & & $\begin{array}{c}\mathrm{A}=1.41 \mathrm{LMH} / \mathrm{bar} \\
\mathrm{B}=1.62 \mathrm{LMH} \\
\mathrm{S}=0.865 \mathrm{~mm} \\
\mathrm{R}=92.23\end{array}$ & [152] \\
\hline
\end{tabular}




\subsection{Performance of TFC-D membranes}

One of the most important developments in the production of FO membranes is producing doubleskinned membranes to solve the problems caused by fouling and concentration polarization without changing the AL-DS orientation (because in this direction a higher flux can be achieved) [180]. Both laboratory studies and modeling have proven the effectiveness of TFC-D's [181-184]. In FO operation, one of the skin layers is in contact with the draw solution, acting as the semipermeable membrane, while the other layer is in contact with the feed solution, preventing the foulants from entering the porous substrate. [181]. Wang et al. [182] were the first to develop a double-skinned membrane from CTA for FO. Fig. 23 shows the schematic image of a doubleskinned FO membrane. $A_{\text {draw }}$ is the water permeability of the active layer of the draw solution side (draw active layer), $B_{\text {draw }}$ is the salt permeability of the active layer of the draw side, $A_{\text {feed }}$ is feed layer water permeability, $B_{\text {feed }}$ is salt permeability of feed layer, $\pi_{s / d}$ and $C_{s / d}$ are the osmotic pressure and solute concentration at the interface between draw active layer and the substrate, respectively, $\pi_{s / f}$ and $C_{s / f}$ are the osmotic pressure and the solute concentration at the interface between the substrate and the feed active layer, respectively, and finally $P_{\text {support }}$ is the hydraulic pressure in the substrate layer [181].

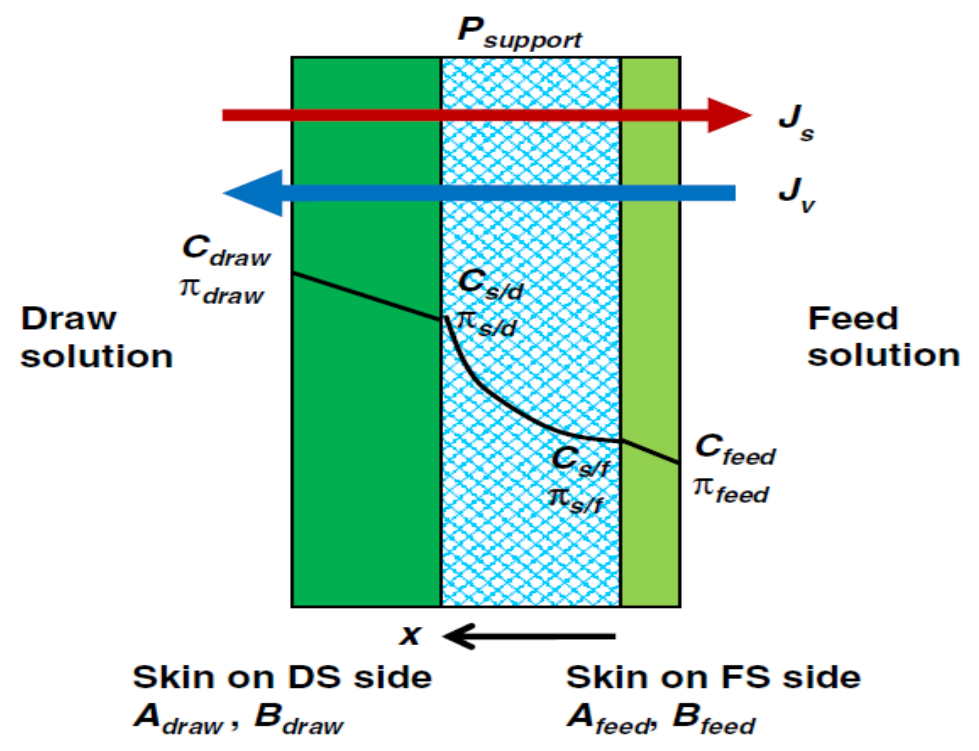

Fig 23: schematic of Double-skinned membrane [181]

\subsubsection{Inner active layer RO polyamide and outer skin layer NF polyethyleneimine}

Fang et al. [180] developed a new TFC membrane in the form of a TFC-D. An active layer similar to the typical active layer used in RO and another active layer similar to a NF active layers were formed onto the two sides of poly (amide-imide) (PAI) hollow fiber substrate. The inner active layer (RO) was made from polyamide by interfacial polymerization, and the outer respectively. Additionally, two PAI hollow fiber substrates, PAI\#1 and PAI\#2 were spun by 
changing the bore fluid flow rate and air gap using constant spinning dope composition, as shown in Table 8. Using these substrates, three TFC-D hollow fibers were fabricated, i.e. $\mathrm{PAI} \# 1-\mathrm{NF} / \mathrm{RO}, \mathrm{PAI} \# 1-\mathrm{RO} / \mathrm{NF}$ and PAI\#2-RO/NF. The membrane codes indicate the substrate used and the sequence of skin layer formation, e.g. NF/RO means the outer active layer (NF) was formed first, followed by the inner layer (RO) formation.

Table 8: Spinning conditions and parameters [180]

\begin{tabular}{lcc}
\hline \multicolumn{1}{c}{ Parameters } & PAI\#1 & PAI\#2 \\
\hline Dope composition (PAI/LiCl/NMP) (wt.\%) & $14 / 4 / 82$ & $14 / 4 / 82$ \\
\hline Dope flow rate $(\mathrm{g}$ min-1) & 6.0 & 6.0 \\
\hline Bore fluid (NMP/H2O) $(\mathrm{vol} . \%)$ & $25 / 75$ & $25 / 75$ \\
\hline Bore fluid flow rate $(\mathrm{mL}$ min-1) & 7.0 & 6.0 \\
\hline Air gap $(\mathrm{cm})$ & 5.0 & 2.0 \\
\hline Take up speed & Free fall & Free fall \\
\hline External coagulant & Tap water & Tap water \\
\hline Spinning temperature $\left({ }^{\circ} \mathrm{C}\right)$ & 23 & 23 \\
\hline Spinneret diameter $(\mathrm{mm})$ & 1.5 & 1.5 \\
\hline ID of bore fluid needle $(\mathrm{mm})$ & 0.7 & 0.7 \\
\hline
\end{tabular}

The morphology of the substrates of the membranes was studied by SEM. Fig. 24 shows SEM 11
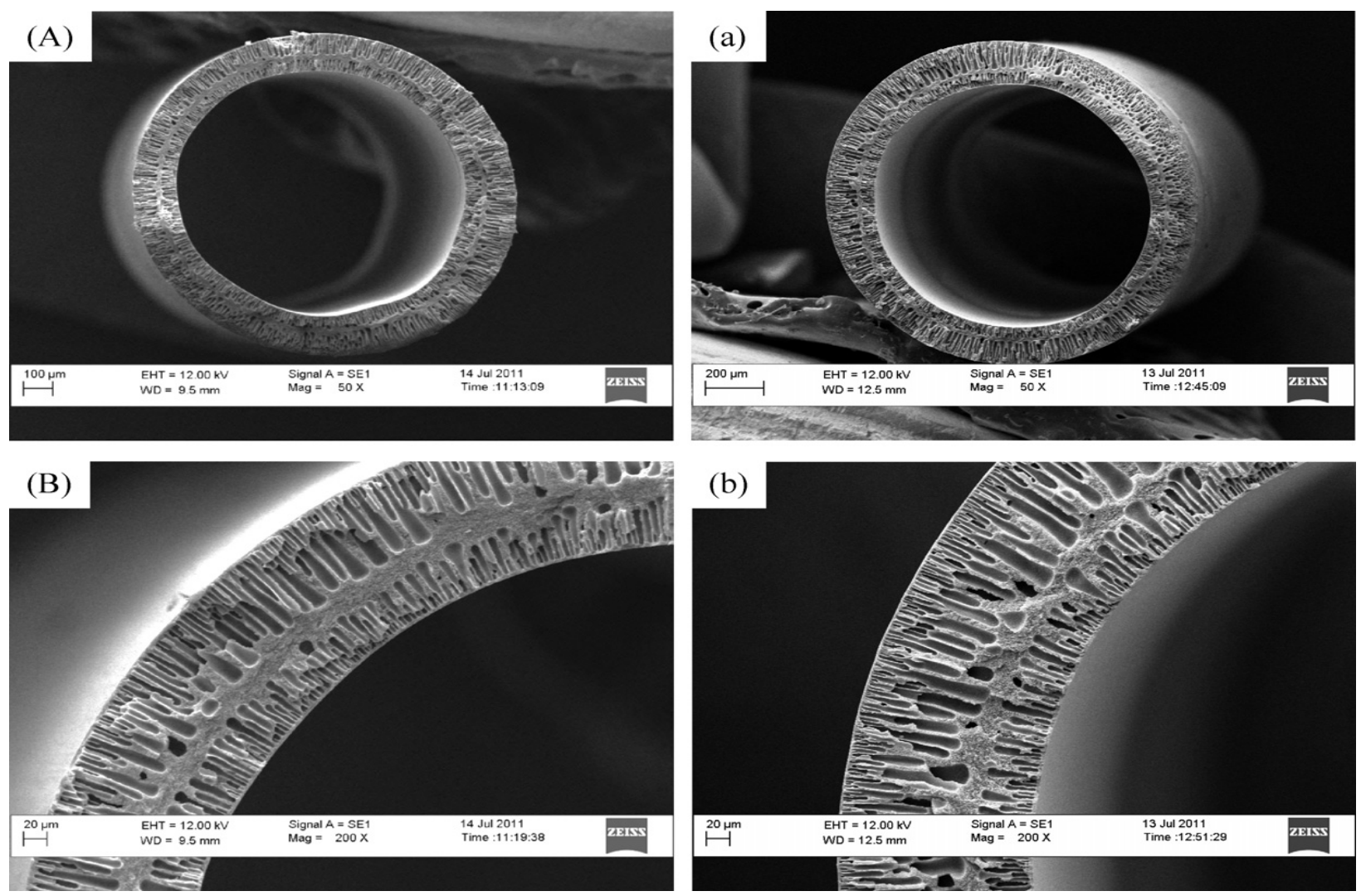

Fig 24: Cross-section morphologies of PAI hollow fiber substrates: (A) PAI\#1 at 50×; (B) PAI\#1 enlarged at 200×;

(a) PAI\#2 at 50×; (b) PAI\#2 enlarged at 200× [180] 
1 Fig. 24 illustrates that the cross-section of the substrates is divided into three regions, including a 2 narrow area of the sponge-like structure at the middle which was sandwiched between two wider 3 finger-like sections. The middle layer of the substrate PAI\#2 is thinner than that of PAI\#1 substrate 4 due to its shorter air gap. The shorter air gap, caused the spun solution to come into contact with 5 the external coagulant (water) sooner [180]. Indeed, before entering the spun fiber into the external 6 coagulation bath, the presence of water vapor in the ambient air induced a slight phase inversion 7 at the outer surface. This led to increase the viscosity at the outer surface, hence, a much thicker 8 skin was obtained [185]. Because the bore fluid non-solvent was weaker than the external 9 coagulant fluid, the finger-like structure developed from the outer surface was deeper than that of 10 the inner surface one. Hollow fibers with the larger fraction of finger-like layer is more desirable 11 due to the higher porosity hence higher membrane productivity.

12 The performance of the three TFC-D hollow fiber membranes was studied. They used $\mathrm{NaCl}$ draw 13 solution of 0.5 to 2 molar concentrations and deionized water as the feed in both AL-DS and AL14 FS orientation, as the results are shown in Fig. 25. From the figure, PAI\#2-RO/NF exhibits the highest water flux in both orientations likely due to the higher porosity of PAI\#2 substrate. 16 Comparing AL-DS and AL-FS orientation, AL-DS demonstrated higher water flux, as expected. $17 \mathrm{PAI}+2-\mathrm{RO} / \mathrm{NF}$ in AL-DS orientation also showed the lowest solute flux/water flux $\left(J_{s} / J_{v}\right)$ ratio. 18 Finally, by comparing the performance of the fabricated membranes in this work with those of other studies, Fang et al. concluded that membranes made with two active layers have better performance than the membranes with only one active layer when the feed solution containing 21 bivalent cations causes membrane fouling. In addition, it was concluded that the TFC-D hollow fiber membrane demonstrated better performance than the TFC-D flat sheet membranes. It is 23 presumably due to higher intrinsic properties of hollow fiber membrane in comparison to flat sheet 24 membrane as a substrate [180]. 
(a) Water flux for AL-DS orientation

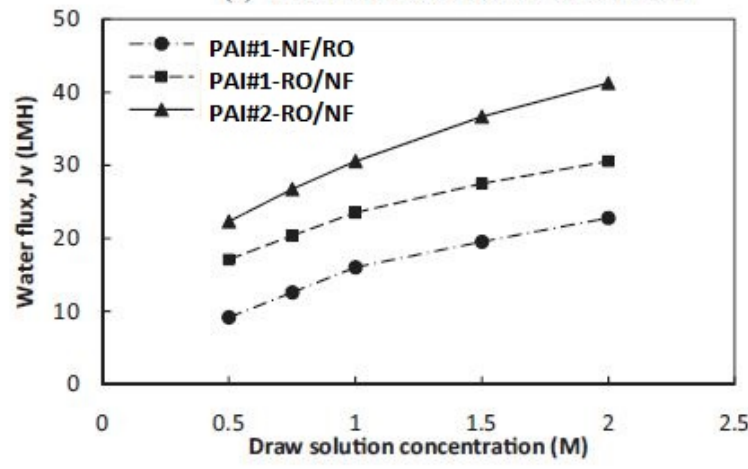

(b) Water flux for AL-FS orientation

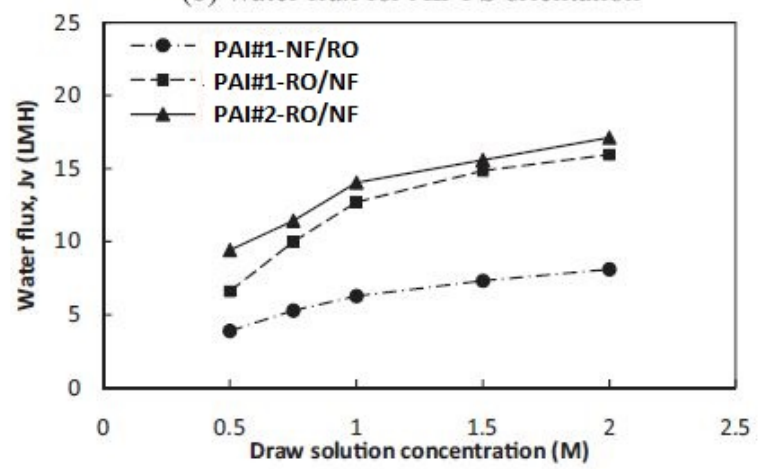

(c) $\mathrm{J}_{\mathrm{s}} / \mathrm{J}_{\mathrm{v}}$ for AL-DS orientation

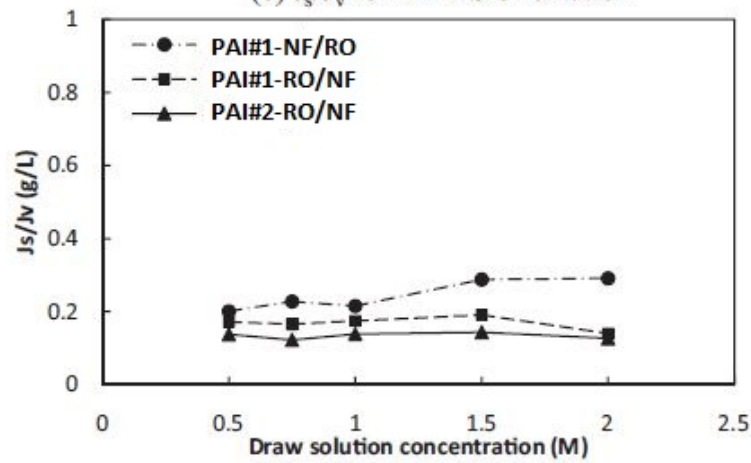

(d) $J_{s} / J_{v}$ for AL-FS orientation

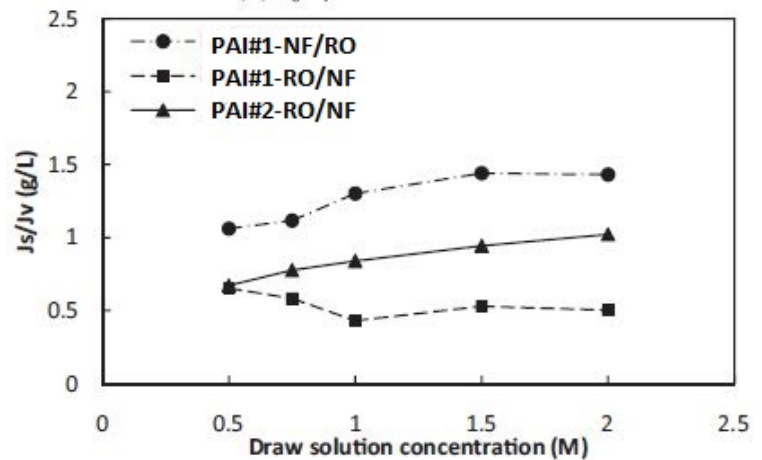

Fig 25: FO performance of TFC-D hollow fiber membranes: Draw solution: 0.5-2.0 M NaCl; feed: DI water. (a) Water flux for AL-DS (PRO) orientation, (b) water flux for AL-FS(FO) orientation, (c) Js/Jv for AL-DS orientation and (d) Js/Jv for AL-FS orientation [180]

Fig. 25 also depicts the effect of the sequence of the inner and outer layer formation on FO performance, i.e. the water flux of PA\#1-NF/RO is lower than PA\#1-RO/NF. When NF membrane is first formed on the outer layer with chemical modification by PEI, a large number of free amine groups from PEI was attached onto the cross-linked outer skin. During the formation of the second skin (RO) on the inner surface by interfacial polymerization of TMC and MPD, most likely the excess of TMC monomers permeated through the substrate and contacted with the free amines of PEI left at the outer layer, resulting in denser outer layer. On the other hand, when interfacial polymerization was occurred first, the excess TMC monomers were quickly altered to carboxylic acid and their reactivity was reduced before subsequent PEI skin layer was fabricated. Consequently, the fabrication of the two dense skin layers has less interference with each other [180]. It is also possible that no monomers remain unreacted after the interfacial polymerization.

\subsubsection{Both skin layers from polyamide}

Han et al. [184] developed a hollow fiber membrane with two polyamide active layers formed on the two sides of PES substrate to control the internal fouling and concentration polarization. The active layer on the inner surface acted as the selective layer, while the outer active layer just prevented foulants from entering into the substrate. The average pore size of the second active 
layer is $0.7 \mathrm{~nm}$ with narrow pore size distribution, which is sufficient to prevent the foulants from entering the substrate pore. Two feeds, wastewater and deionized water, were used together with $1 \mathrm{M} \mathrm{NaCl}$ as draw solution. When the membrane with one selective layer is applied in AL-DS (PRO) orientation, the substrate is in contact with the feed and thus foulants can easily enter the porous substrate (see Fig.26 (a)), resulting in severe internal concentration polarization and fouling, which reduces the flux. As a result, removing the foulants from the membrane becomes more challenging. The TFC-D could prevent the foulant intrusion into the substrate, as shown in 8 Fig. 26 (b). Although the external concentration polarization (ECP) is intensified in this case because of the second active layer, the negative effect of ECP is less severe than ICP in FO. Moreover, the foulants deposited on top of the polyamide layer could be easily washed away [184].

(a)

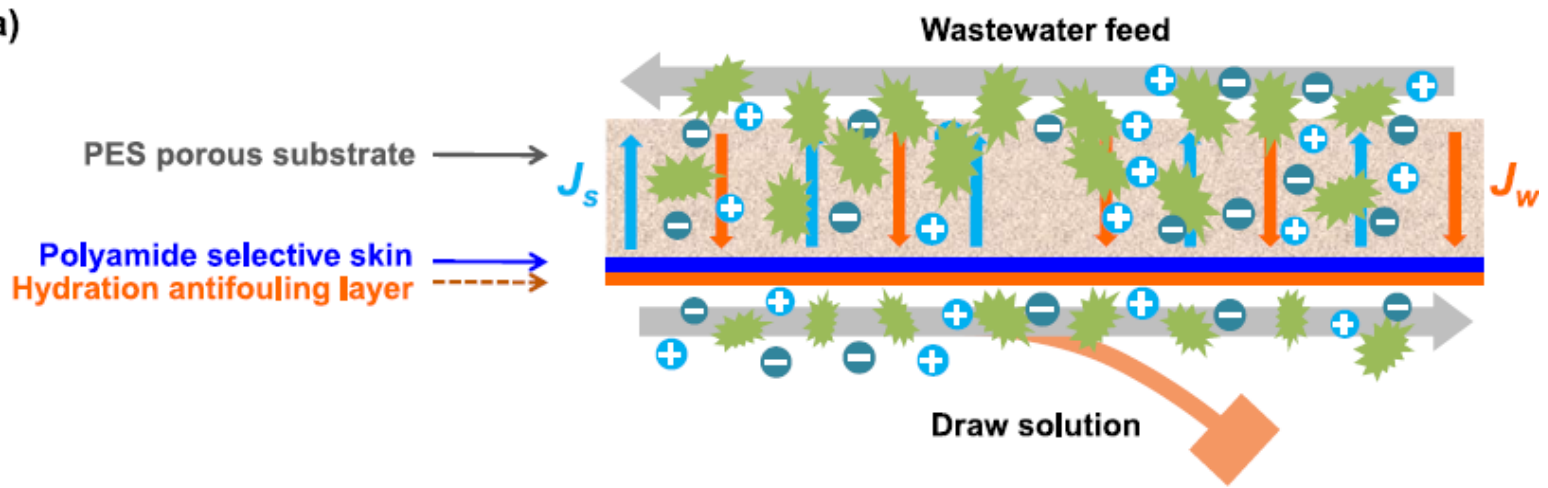

(b)

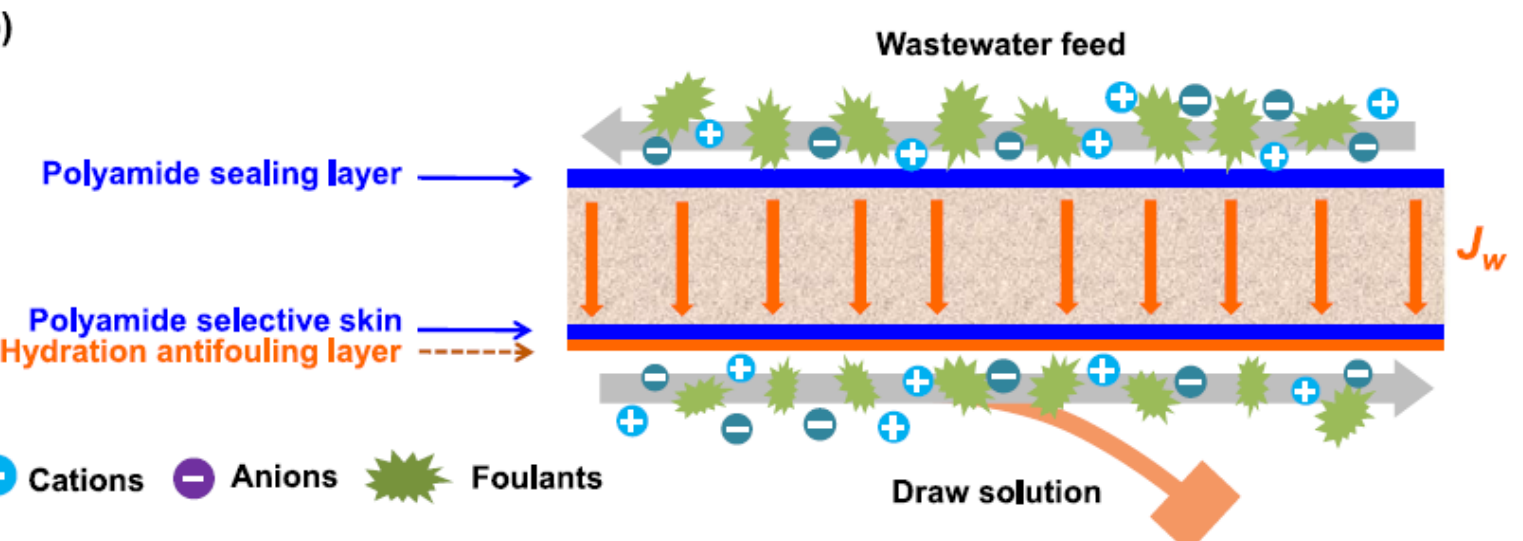

Fig 26: Schematic of fouling phenomena: (a) the conventional TFC membrane and (b) the newly developed TFC-D membrane in FO under the PRO mode [184].

Table 9 shows the review of literature on the development of TFC-D membranes.

\subsection{Issues and implications of TFC-D membranes}

Although, TFC-D membranes show excellent properties especially regarding reverse salt flux and fouling propensity, these membranes show low water flux compared with the normal TFC membranes i.e. with single skin. Methods such as IP, layer-by-layer polyelectrolyte deposition and PDA deposition are applied to produce double-skinned FO membranes. For achieving high water 
1 flux of TFC-D membranes, it is suggested to use an RO-like active layer on one side and an NF

2 active layer on the other side. Fabricating two RO-like active layer on the two side can drastically

3 decrease the water flux. It is also recommended to apply nanomaterial in one or both of the active 4 layers to compensate the decline in water flux. 
2 Table 9: Summary of Double-skinned TFC FO membranes a

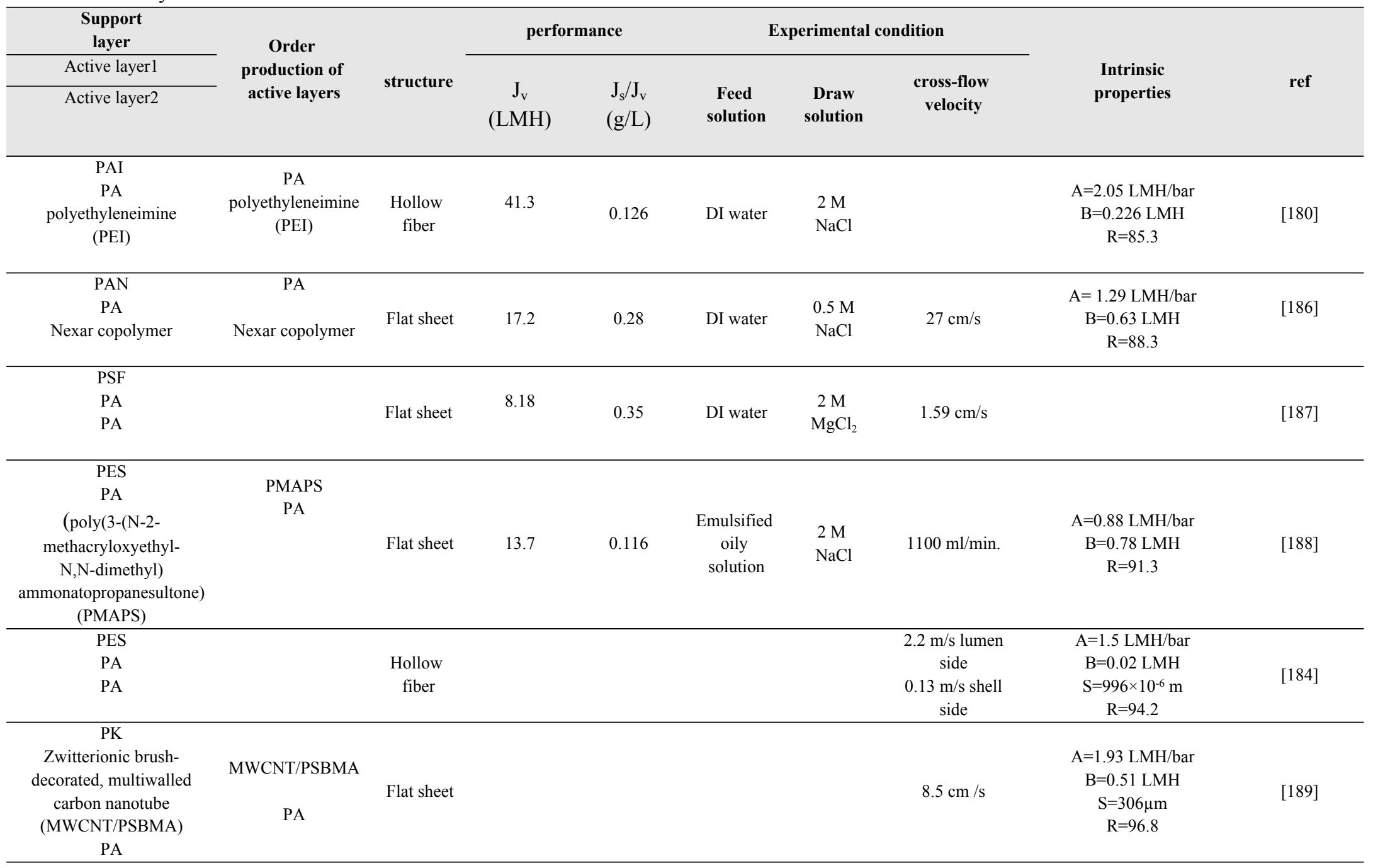

a: data is reported in AL-DS orientation 


\section{Commercial FO membranes}

Despite many membranes that have been designed by various scientific groups specifically for FO process, it seems FO technology still needs membranes of higher performance. Fortunately, however, some of the membranes have already been commercialized. The commercial membranes are mostly in flat sheet form [37]. Table 10 summarizes the current commercial FO membranes. Although, some papers [190] have mentioned several other companies as FO membrane supplier, we could not successfully find reliable information from their websites.

8

Table 10: Status of the current commercial FO membranes

\begin{tabular}{lcccc}
\hline Supplier/Manufacturer & Status & $\begin{array}{c}\text { System } \\
\text { supply }\end{array}$ & $\begin{array}{c}\text { Membrane and } \\
\text { Configuration }\end{array}$ & Primary application \\
\hline HTI & Commercial & Yes & SWo (CA, TFC) & Various \\
\hline Aquaporin A/S & Commercial & No & SWo, HF (Aquaporin) & $\begin{array}{c}\text { FO, osmotic } \\
\text { concentration }\end{array}$ \\
\hline Modern Water & Commercial & Yes & SWo & Seawater FO \\
\hline Oasys Water & Commercial & Yes & SWo (TFC) & Brine concentration, ZLD \\
\hline Porifera & Commercial & Yes & SWo (TFC) & Various \\
\hline Trevi Systems & Commercial & Yes & SWo & Seawater FO \\
\hline Green Centre Canada & Development & No & SWo & Seawater FO \\
\hline Idaho National Lab & Development & No & NA & Reverse osmosis \\
\hline Fluid Technology solutions & Commercial & NA & SWo (CTA) & Wastewater treatment \\
\hline IDE Technologies & Precommercial & Yes & SWo & Press.-retarded osmosis \\
\hline $\begin{array}{l}\text { NA: not available; SWo :spiral wound, HF: hollow fiber, ZLD: Zero liquid discharge } \\
\text { a Demonstration-scale FO membrane treatment system available (yes/no) }\end{array}$ & & & & \\
\hline
\end{tabular}

In contrast to the membranes listed in Table 1 HTI Co. commercialized the FO membranes for the first time. Unfortunately, they no longer supply their products [46]. However, because of their historic importance, the details of their membranes are given below.

\subsection{HTI membrane}

Ren et al. [191] characterized and tested the performance of a TFC membrane developed by HTI Co. The FESEM images of the active layer and the substrate surface at different magnifications are shown in Fig. 27. The active layer has a ridge and valley morphology which is a typical feature of polyamide active layers formed by interfacial polymerization. The active layer seems uniform and defect-free. The membrane substrate is porous with pore sizes from 100 to $600 \mathrm{~nm}$. Moreover, they soaked the TFC membrane in 50\% wt. isopropyl alcohol (IPA) solution at room temperature for 5 minutes. Then, isopropyl alcohol was completely washed out with deionized water in which the membrane was stored at a temperature of $5{ }^{\circ} \mathrm{C}$. The performance of this soaked membrane was compared with that of the non-soaked TFC membrane. The water and salt permeability increased in by soaking. When the membrane was soaked in IPA, no chemical reaction occurred between polyamide and IPA, but the molecules of low molecular weight were removed from the active 
layer, thereby forming a more open-structured polyamide active layer. Also, in the presence of 2 IPA, the swelling of polyamide chains has increased due to the hydrogen bonding between IPA 3 and polyamide and the affinity of IPA to the nonpolar part of polyamide. Therefore, the interaction 4 between the polymer chains was weakened in the active layer and the chains became more 5 flexible, which in turn increased the pore size. The presence of larger pores and open structure 6 increase simultaneously the permeability of water and salt.
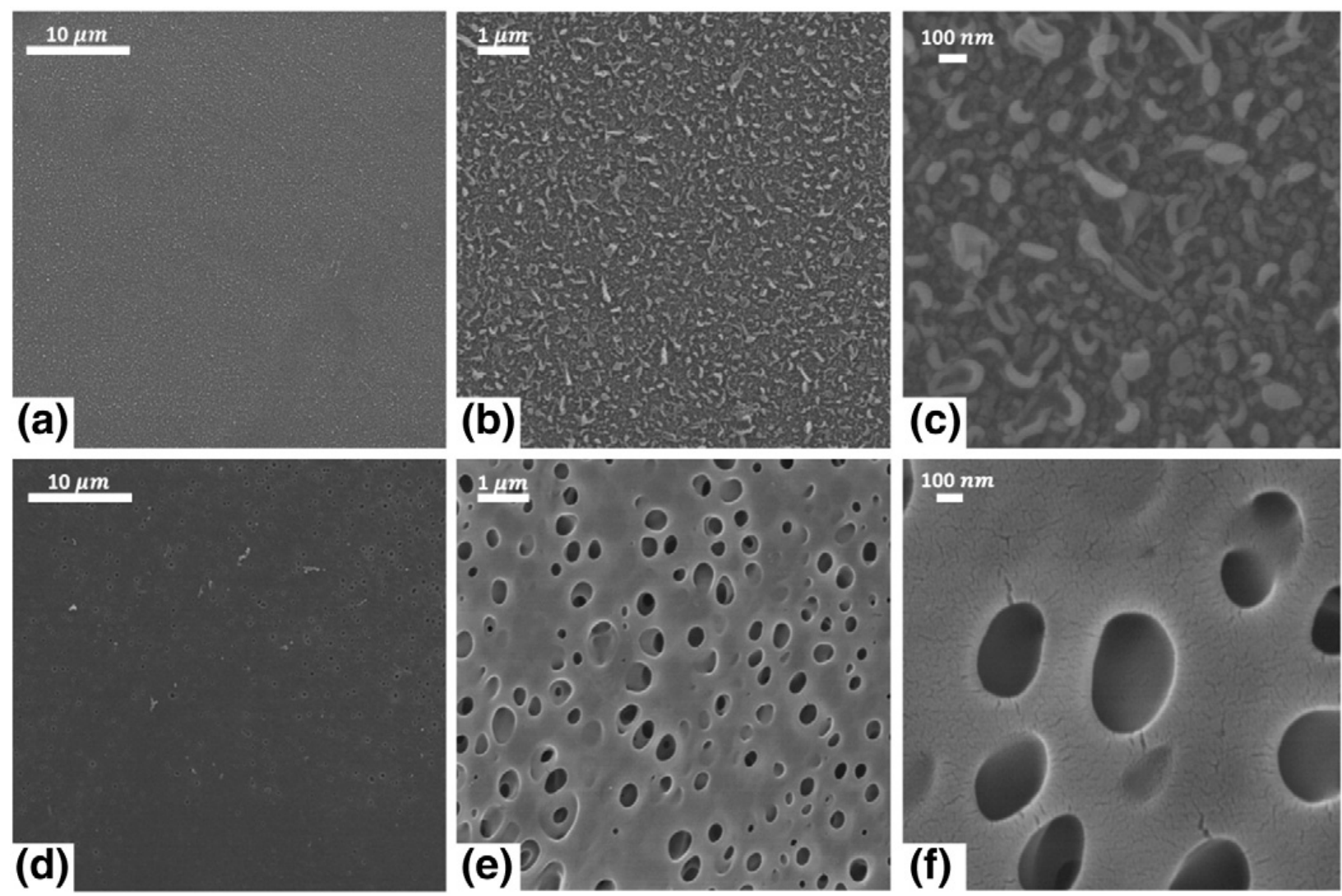

Fig 27: Top surface SEM images ( $a, b$ and $c$ ) and bottom surface FESEM images (d, e and $f$ ) of TFC membrane developed by HTI Co. at magnifications of (a and d) 2000×, (b and e) 10,000×, and (c and f) 50,000× [191]

\subsection{Aquaporin A/S membrane}

Ren el al. [192] reported their research on the performance of the hollow fiber FO membrane supplied by Aquaporin A/S. The small-scale modules were applied for testing the membrane under various testing conditions. They incorporated aquaporin into the selective layer of the hollow fibers at the lumen surface. These membranes presented excellent FO performance. Fig. 28 illustrates the cross-sectional morphology of the membranes at different magnifications. The entire structure of the hollow fiber membranes was sponge-like structure. Sponge-like structure would be useful for long term operation because of its higher mechanical stability. 

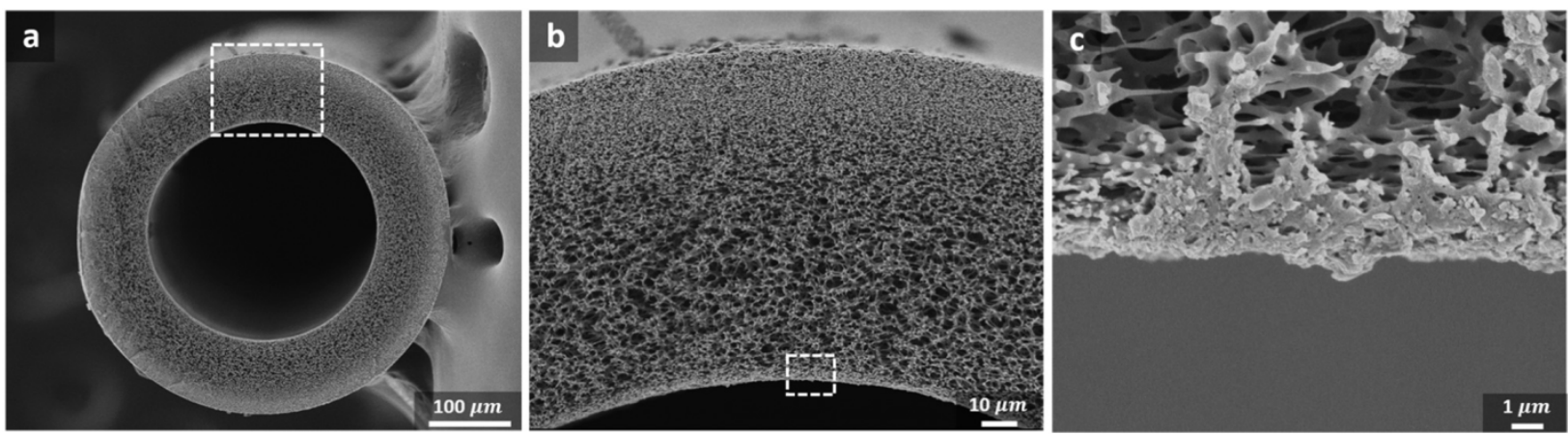

Fig. 28. Cross-section FESEM images of a hollow fiber membrane at (a) $170 \times$; (b) $800 \times$; (c) $5000 \times$.

Table 11 shows the results on the water flux and the specific reverse salt flux of the current commercial FO membranes.

Table 11: Summary of the studies on the current commercial FO membranes

\begin{tabular}{|c|c|c|c|c|c|c|c|c|}
\hline \multirow{3}{*}{$\begin{array}{l}\text { Membrane } \\
\text { supplier }\end{array}$} & \multirow[b]{3}{*}{ structure } & \multicolumn{4}{|c|}{ performance } & \multicolumn{2}{|c|}{ Experimental condition } & \\
\hline & & \multicolumn{2}{|c|}{ AL-FS(FO) } & \multicolumn{2}{|c|}{ AL-DS(PRO) } & \multirow[b]{2}{*}{$\begin{array}{c}\text { Feed } \\
\text { solution }\end{array}$} & \multirow[b]{2}{*}{$\begin{array}{r}\text { Draw } \\
\text { solution }\end{array}$} & \\
\hline & & $\begin{array}{c}\mathrm{J}_{\mathrm{v}} \\
(\mathrm{LMH})\end{array}$ & $\begin{array}{l}\mathrm{J}_{\mathrm{s}} / \mathrm{J}_{\mathrm{v}} \\
(\mathrm{g} / \mathrm{L})\end{array}$ & $\begin{array}{c}\mathrm{J}_{\mathrm{v}} \\
(\mathrm{LMH})\end{array}$ & $\begin{array}{c}\mathrm{J}_{\mathrm{S}} / \mathrm{J}_{\mathrm{V}} \\
(\mathrm{g} / \mathrm{L})\end{array}$ & & & \\
\hline HTI & Flat sheet & 21.10 & 0.13 & 31.61 & 0.45 & DI water & $1 \mathrm{M} \mathrm{NaCl}$ & [191] \\
\hline $\begin{array}{c}\text { Prewetted } \\
\text { HTI }\end{array}$ & Flat sheet & 23.03 & 0.27 & 46.69 & 0.52 & DI water & $1 \mathrm{M} \mathrm{NaCl}$ & [191] \\
\hline Toyobo & Hollow fiber & 8 & 0.07 & 15 & N/A & DI water & $1 \mathrm{M} \mathrm{NaCl}$ & [193] \\
\hline $\begin{array}{c}\text { Aquaporin } \\
\mathrm{A} / \mathrm{S}\end{array}$ & Hollow fiber & 13.2 & 0.14 & 21 & .18 & DI water & $1 \mathrm{M} \mathrm{NaCl}$ & {$[192]$} \\
\hline
\end{tabular}

\section{Proposing roadmap, concluding remarks and future directions}

In this paper, recent developments in TFC FO membranes, as an emerging process considering its challenges and capabilities were reviewed. A number of studies were considered on the development and design of the membranes and the challenges during its development stages were discussed. TFC FO membranes should have a robust but thin substrate and a well-organized active layer with a high-water permeability and selectivity which can help to make FO as a practical competitive process in desalination and water treatment applications.

Based upon Fig. 1, it is obvious that up to this point three major methods have been developed by researchers to fabricate TFC FO membranes. In order to have a correct understanding of the superiority of each of the membrane groups, Fig. 29 as a general performance curve and a road map for TFC-FO membranes was generated. The data for TFC-A membranes, TFC-N membranes and TFC-D membranes were taken from Tables 1 and 3, 6 and 9, respectively. Fig. 29a depicts FO reverse salt flux $/ \Delta \pi\left(J_{S} / \Delta \pi\right)$ versus FO water flux $/ \Delta \pi\left(J_{v} / \Delta \pi\right)$ almost for all the FO membranes reviewed in this work. Clearly, several important facts are shown by the figure. First, based on the available data, there is a general trend between $J_{S} / \Delta \pi$ and $J_{v} / \Delta \pi$, i.e. reverse salt flux is directly related to water flux. Disregarding the membrane preparation methods, this is true for the all three 
groups of membranes collected in this study. Second, the increasing trend is accompanied by a flat

2 slope, which is corresponding to a certain amount of the $J_{v} / \Delta \pi$ for each group. The trends of 3 substrate modified TFC-N's and TFC-A's are very similar to each other. Third, the addition of 4 nanoparticles to the active layer structure as active layer modified TFC-N's shows two different 5 features corresponding to the $J_{v} / \Delta \pi$ values before and after $\sim 0.4 \mathrm{LMH} / \mathrm{bar}$. At low $J_{v} / \Delta \pi$ the overall 6 results have been enhanced in terms of controlling the reverse salt flux compared to normal TFC 7 membranes, however, at higher $J_{v} / \Delta \pi$ the trend is reverse and the reverse salt flux increased. The 8 first part is most likely because of low nanoparticle loadings, appearing appropriate interaction 9 between the PA and the nanoparticle, whilst the second part is most probably due to the high 10 nanoparticle loadings that resulted in particle agglomeration and pinhole formation in the active 11 layer. Therefore, clearly further carefully designed studies are needed to stablish the nanoparticle 12 application in TFC-N membranes to shift the above inflection point toward higher $J_{v} / \Delta \pi$ values. 13 Interestingly, from Fig. 29, as an important progress in the trend of synthesis of FO membranes, 14 TFC-D membranes meaningfully possess the potential of controlling the reverse solute diffusion, 15 which this in turn is associated with a significant decrease in ICP. Fig. 29b illustrates the 16 meaningful gap between TFC-D membranes and TFC-A and TFC-N membranes in terms of $J_{S} / J_{v}$ 17 versus $J_{v} / \Delta \pi$. Fortunately, the trend is quite descending, whereas for the rests is ascending. 18 Therefore, this conclusion can be drawn that working on active layer modified TFC-Ns and 19 double-skinned membranes TFC-D, and investigating the correlation between the nanoparticles 20 and the membrane performance will be an important focus area in order to commercialize FO 21 membranes.

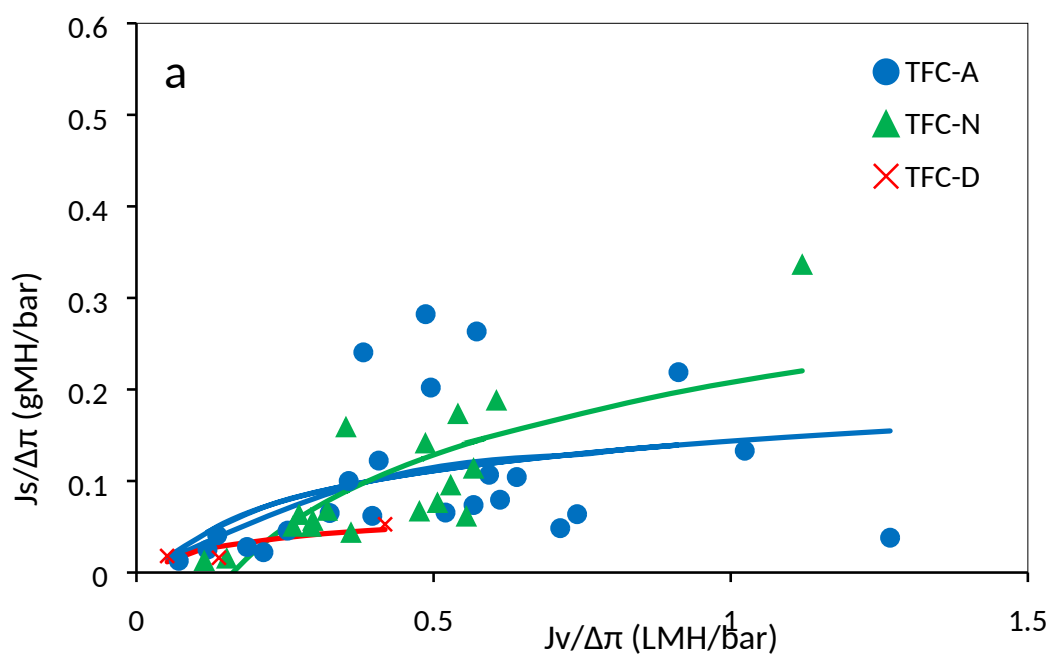




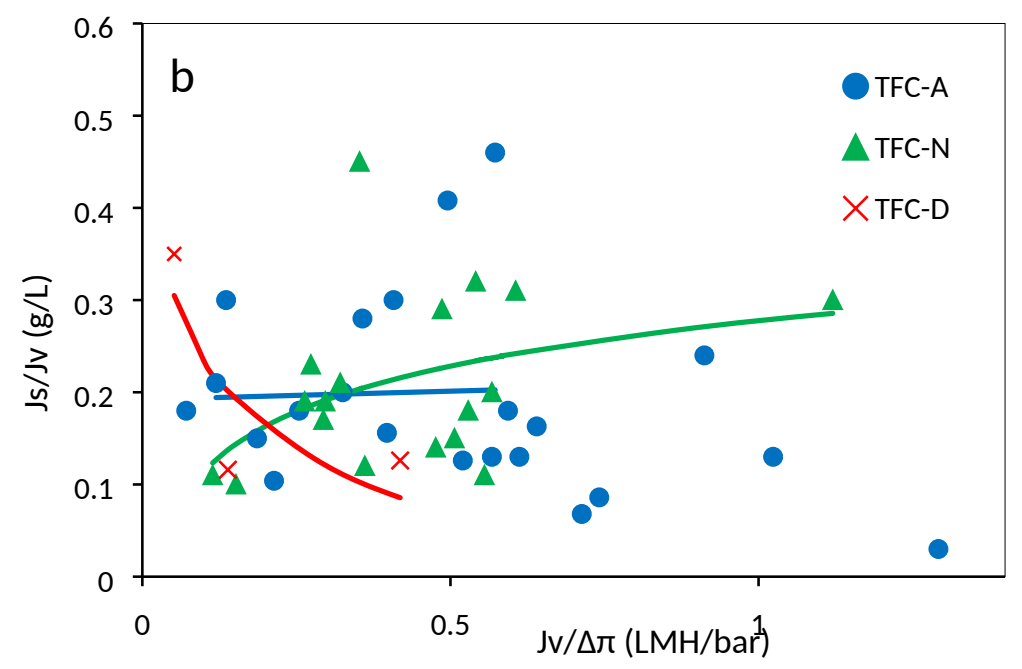

Fig. 29: Reverse salt flux versus water flux for the FO membranes reviewed in this work (TFC-A and TFC-N: ALFS mode, TFC-D: AL-DS mode) (data points are taken from Tables 1, 3,6 and 9)

According to this review, it can be concluded that the most studies on the production of TFC membranes have been conducted with deionized water as feed and a low concentration of draw solutions. For a better understanding of the membrane performance, the lab scale test conditions such as the composition of feed and draw solutions should be preferentially close to that of actual conditions. For achieving better understanding of the performance of the FO membranes Hao et al. [114] recommended that, instead of permeability-selectivity, permeability-selectivityantifouling trade-off must be considered.

In the majority of the studies, the commercial TFC FO membranes are of the flat sheet form. Considering the pronounced superiority of the hollow fiber membranes over the flat sheet membranes, it is advisable to perform more laboratory research and modeling on the application of hollow fiber membranes in FO. Also, more studies should be performed on the less considered conditions of FO process i.e. temperature of the feed and different draw solutions. The lack of study on TFC-N's is quite clear. However, the present fabricated TFC-N's exhibit high-water flux, they suffer from the shortcoming of high reverse solute diffusion. Hence, more research is necessary for controlling this undesired aftermath before commercialization of TFC-N membranes. Based on the present review, TFC-D's have a brilliant future. Therefore, more studies should be conducted on them because of their prominent potential for controlling ICP and membrane fouling. Moreover, further studies are required to reveal the role of the nanoparticles incorporating in both skins simultaneously on the performance of the membrane. Generally, selection of suitable nanomaterials is a key-parameter in TFC-N membrane formation. This selection depends on the composition of feed and draw solution. Existence of nanoscale fillers can enhance the free volume by disrupting the polymer main chains causing to higher water diffusivity enhancement [194]. From the open literature four main category of nano-particles can be observed which are suggested to be studied, namely: i) Microstructural design such as: MOF, GO, zeolitic imidazolate framework (ZIF), CNTs , single wall carbon nanotubes (SWCNTs), etc.), ii) Co- 
synthesis of advanced fillers such as: ZIF-8@GO, iii) Novel organometallic nanostructures such as: e.g. coordinated ligands, porous coordination polymers (PCPs), metal organic polyhedras (MOPs), ion-loaded macromolecules microporous, organic/inorganic hybrids, etc. and iv) Porous organic frameworks (POFs) for instance covalent organic frameworks (COFs), covalent triazine based frameworks (CTFs), porous aromatic frameworks (PAFs) and conjugated microporous polymers (CMPs) [194].

\section{Acknowledgements}

This research was funded by the Iran National Science Foundation (INSF) for the project entitled, "Development and fabrication of thin film composite and nanocomposite hollow fiber membrane for forward osmosis process" (Grant number: 98011635). The authors also gratefully acknowledge Sustainable Membrane Technology Research Group (SMTRG), Faculty of Petroleum, Gas and Petrochemical Engineering (FPGPE), Persian Gulf University (PGU), Iran for their financial support. In addition, the authors would like to thank Advanced Membrane Technology Research Centre (AMTEC), Universiti Teknologi Malayia (UTM) for their spiritual support.

\section{References}

[1] N. Akther, A. Sodiq, A. Giwa, S. Daer, H. Arafat, S. Hasan, Recent advancements in forward osmosis desalination: a review, Chemical Engineering Journal, 281 (2015) 502-522.

[2] K. Lutchmiah, A. Verliefde, K. Roest, L.C. Rietveld, E.R. Cornelissen, Forward osmosis for application in wastewater treatment: a review, Water research, 58 (2014) 179-197.

[3] D.L. Shaffer, N.Y. Yip, J. Gilron, M. Elimelech, Seawater desalination for agriculture by integrated forward and reverse osmosis: Improved product water quality for potentially less energy, Journal of membrane science, 415 (2012) 1-8.

[4] Z.-Y. Li, V. Yangali-Quintanilla, R. Valladares-Linares, Q. Li, T. Zhan, G. Amy, Flux patterns and membrane fouling propensity during desalination of seawater by forward osmosis, Water research, 46 (2012) 195-204.

[5] S. Zou, M. Qin, Z. He, Tackle reverse solute flux in forward osmosis towards sustainable water recovery: reduction and perspectives, Water research, 149 (2019) 362-374.

[6] L. Chekli, S. Phuntsho, H.K. Shon, S. Vigneswaran, J. Kandasamy, A. Chanan, A review of draw solutes in forward osmosis process and their use in modern applications, Desalination and Water Treatment, 43 (2012) 167-184.

[7] F. Lotfi, B. Samali, D. Hagare, Cleaning efficiency of the fouled forward osmosis membranes under different experimental conditions, Journal of Environmental Chemical Engineering, 6 (2018) 4555-4563.

[8] R. Alnaizy, A. Aidan, M. Qasim, Copper sulfate as draw solute in forward osmosis desalination, Journal of Environmental Chemical Engineering, 1 (2013) 424-430.

[9] J. Mulder, Basic principles of membrane technology, Springer Science \& Business Media, 2012.

[10] A. Sotto, A. Rashed, R.-X. Zhang, A. Martínez, L. Braken, P. Luis, B. Van der Bruggen, Improved membrane structures for seawater desalination by studying the influence of sublayers, Desalination, 287 (2012) 317-325. 
[11] F.M. Munshi, J. Church, R. McLean, N. Maier, A.A. Sadmani, S.J. Duranceau, W.H. Lee, Dewatering algae using an aquaporin-based polyethersulfone forward osmosis membrane, Separation and Purification Technology, 204 (2018) 154-161.

[12] J. Kessler, C. Moody, Drinking water from sea water by forward osmosis, Desalination, 18 (1976) 297306.

[13] W.L. Ang, A.W. Mohammad, D. Johnson, N. Hilal, Forward osmosis research trends in desalination and wastewater treatment: A review of research trends over the past decade, Journal of Water Process Engineering, 31 (2019) 100886.

[14] J. Beh, B. Ooi, J. Lim, E. Ng, H. Mustapa, Development of high water permeability and chemically stable thin film nanocomposite (TFN) forward osmosis (FO) membrane with poly (sodium 4styrenesulfonate)(PSS)-coated zeolitic imidazolate framework-8 (ZIF-8) for produced water treatment, Journal of Water Process Engineering, 33 (2020) 101031.

[15] D. Emadzadeh, T. Matsuura, M. Ghanbari, A.F. Ismail, Hybrid forward osmosis/ultrafiltration membrane bag for water purification, Desalination, 468 (2019) 114071.

[16] Y. Huang, P. Cay-Durgun, T. Lai, P. Yu, M.L. Lind, Phenol removal from water by polyamide and $\mathrm{AgCl}$ mineralized thin-film composite forward osmosis membranes, Industrial \& Engineering Chemistry Research, 57 (2018) 7021-7029.

[17] J.E. Kim, S. Phuntsho, S.M. Ali, J.Y. Choi, H.K. Shon, Forward osmosis membrane modular configurations for osmotic dilution of seawater by forward osmosis and reverse osmosis hybrid system, Water research, 128 (2018) 183-192.

[18] C.F. Wan, Y. Cui, W.X. Gai, Z.L. Cheng, T.-S. Chung, Nanostructured Membranes for Enhanced Forward Osmosis and Pressure-Retarded Osmosis, in: Sustainable Nanoscale Engineering, Elsevier, 2020, pp. 373394.

[19] L. Deng, Q. Wang, X. An, Z. Li, Y. Hu, Towards enhanced antifouling and flux performances of thin-film composite forward osmosis membrane via constructing a sandwich-like carbon nanotubes-coated support, Desalination, 479 (2020) 114311.

[20] H. Guo, Z. Yao, J. Wang, Z. Yang, X. Ma, C.Y. Tang, Polydopamine coating on a thin film composite forward osmosis membrane for enhanced mass transport and antifouling performance, Journal of Membrane Science, 551 (2018) 234-242.

[21] W. Suwaileh, D. Johnson, S. Khodabakhshi, N. Hilal, Superior cross-linking assisted layer by layer modification of forward osmosis membranes for brackish water desalination, Desalination, 463 (2019) 112.

[22] D.J. Johnson, W.A. Suwaileh, A.W. Mohammed, N. Hilal, Osmotic's potential: An overview of draw solutes for forward osmosis, Desalination, 434 (2018) 100-120.

[23] S.M. Ali, J.E. Kim, S. Phuntsho, A. Jang, J.Y. Choi, H.K. Shon, Forward osmosis system analysis for optimum design and operating conditions, Water research, 145 (2018) 429-441.

[24] G. Gwak, B. Jung, S. Han, S. Hong, Evaluation of poly (aspartic acid sodium salt) as a draw solute for forward osmosis, Water research, 80 (2015) 294-305.

[25] M. Sakai, M. Seshimo, M. Matsukata, Hydrophilic ZSM-5 membrane for forward osmosis operation, Journal of Water Process Engineering, 32 (2019) 100864.

[26] T.-S. Chung, X. Li, R.C. Ong, Q. Ge, H. Wang, G. Han, Emerging forward osmosis (FO) technologies and challenges ahead for clean water and clean energy applications, Current Opinion in Chemical Engineering, 1 (2012) 246-257.

[27] M. Giagnorio, F. Ricceri, A. Tiraferri, Desalination of brackish groundwater and reuse of wastewater by forward osmosis coupled with nanofiltration for draw solution recovery, Water research, 153 (2019) 134-143. 
[28] G. Han, C.-Z. Liang, T.-S. Chung, M. Weber, C. Staudt, C. Maletzko, Combination of forward osmosis (FO) process with coagulation/flocculation (CF) for potential treatment of textile wastewater, Water research, 91 (2016) 361-370.

[29] W. Suwaileh, D. Johnson, D. Jones, N. Hilal, An integrated fertilizer driven forward osmosisrenewables powered membrane distillation system for brackish water desalination: A combined experimental and theoretical approach, Desalination, 471 (2019) 114126.

[30] W. Suwaileh, D. Johnson, S. Khodabakhshi, N. Hilal, Cross-linked layer by layer forward osmosis membrane for brackish water desalination, (2019).

[31] W. Suwaileh, D. Johnson, N. Hilal, Brackish water desalination for agriculture: Assessing the performance of inorganic fertilizer draw solutions, Desalination, 456 (2019) 53-63.

[32] M. Qasim, N.A. Darwish, S. Sarp, N. Hilal, Water desalination by forward (direct) osmosis phenomenon: A comprehensive review, Desalination, 374 (2015) 47-69.

[33] H.-g. Choi, A.A. Shah, S.-E. Nam, Y.-I. Park, H. Park, Thin-film composite membranes comprising ultrathin hydrophilic polydopamine interlayer with graphene oxide for forward osmosis, Desalination, 449 (2019) 41-49.

[34] R.V. Linares, Z. Li, S. Sarp, S.S. Bucs, G. Amy, J.S. Vrouwenvelder, Forward osmosis niches in seawater desalination and wastewater reuse, Water research, 66 (2014) 122-139.

[35] L. Zohrabian, N.P. Hankins, R.W. Field, Hybrid forward osmosis-membrane distillation system: Demonstration of technical feasibility, Journal of Water Process Engineering, 33 (2020) 101042.

[36] V. Nayak, M. Jyothi, R.G. Balakrishna, M. Padaki, V.Y. Zadorozhnyy, S. Kaloshkin, 4-aminophenyl sulfone (APS) as novel monomer in fabricating paper based TFC composite for forward osmosis: Selective layer optimization, Journal of Environmental Chemical Engineering, (2020) 103664.

[37] G. Blandin, A.R. Verliefde, J. Comas, I. Rodriguez-Roda, P. Le-Clech, Efficiently combining water reuse and desalination through forward osmosis-reverse osmosis (FO-RO) hybrids: a critical review, Membranes, 6 (2016) 37.

[38] X.-p. Wang, J. Hou, F.-s. Chen, X.-m. Meng, In-situ growth of metal-organic framework film on a polydopamine-modified flexible substrate for antibacterial and forward osmosis membranes, Separation and Purification Technology, 236 (2020) 116239.

[39] D. Li, Y. Yan, H. Wang, Recent advances in polymer and polymer composite membranes for reverse and forward osmosis processes, Progress in Polymer Science, 61 (2016) 104-155.

[40] N.B. Darwish, A. Alkhudhiri, H. AlRomaih, A. Alalawi, M.C. Leaper, N. Hilal, Effect of lithium chloride additive on forward osmosis membranes performance, Journal of Water Process Engineering, 33 (2020) 101049.

[41] J. Herron, Asymmetric forward osmosis membranes, in, Google Patents, 2008.

[42] W. Suwaileh, N. Pathak, H. Shon, N. Hilal, Forward osmosis membranes and processes: A comprehensive review of research trends and future outlook, Desalination, 485 (2020) 114455.

[43] S. Zhao, L. Zou, C.Y. Tang, D. Mulcahy, Recent developments in forward osmosis: opportunities and challenges, Journal of membrane science, 396 (2012) 1-21.

[44] A. Ismail, M. Padaki, N. Hilal, T. Matsuura, W. Lau, Thin film composite membrane-Recent development and future potential, Desalination, 356 (2015) 140-148.

[45] W. Ding, J. Cai, Z. Yu, Q. Wang, Z. Xu, Z. Wang, C. Gao, Fabrication of an aquaporin-based forward osmosis membrane through covalent bonding of a lipid bilayer to a microporous support, Journal of Materials Chemistry A, 3 (2015) 20118-20126.

[46] I. Ndiaye, S. Vaudreuil, T. Bounahmidi, Forward Osmosis Process: State-Of-The-Art of Membranes, Separation \& Purification Reviews, (2019) 1-21.

[47] Q. Liu, G.-R. Xu, Graphene oxide (GO) as functional material in tailoring polyamide thin film composite (PA-TFC) reverse osmosis (RO) membranes, Desalination, 394 (2016) 162-175. 
[48] T.Y. Cath, A.E. Childress, M. Elimelech, Forward osmosis: principles, applications, and recent developments, Journal of membrane science, 281 (2006) 70-87.

[49] M.R. Dalwani, Thin film composite nanofiltration membranes for extreme conditions, University of Twente, 2011.

[50] D.L. Shaffer, J.R. Werber, H. Jaramillo, S. Lin, M. Elimelech, Forward osmosis: where are we now?, Desalination, 356 (2015) 271-284.

[51] G.S. Ibrahim, A.M. Isloor, E. Yuliwati, A Review: Desalination by Forward Osmosis, in: Current Trends and Future Developments on (Bio-) Membranes, Elsevier, 2019, pp. 199-214.

[52] M. Li, V. Karanikola, X. Zhang, L. Wang, M. Elimelech, A self-standing, support-free membrane for forward osmosis with no internal concentration polarization, Environmental Science \& Technology Letters, 5 (2018) 266-271.

[53] J. Zhou, H.-L. He, F. Sun, Y. Su, H.-Y. Yu, J.-S. Gu, Structural parameters reduction in polyamide forward osmosis membranes via click modification of the polysulfone support, Colloids and Surfaces A: Physicochemical and Engineering Aspects, (2019) 124082.

[54] M. Darwish, H. Abdulrahim, A. Hassan, A. Mabrouk, A. Sharif, The forward osmosis and desalination, Desalination and Water Treatment, 57 (2016) 4269-4295.

[55] J.Y. Law, A.W. Mohammad, Employing forward osmosis technology through hybrid system configurations for the production of potable/pure water: a review, Jurnal Teknologi, 79 (2017).

[56] L. Chekli, S. Phuntsho, J.E. Kim, J. Kim, J.Y. Choi, J.-S. Choi, S. Kim, J.H. Kim, S. Hong, J. Sohn, A comprehensive review of hybrid forward osmosis systems: Performance, applications and future prospects, Journal of Membrane Science, 497 (2016) 430-449.

[57] W.L. Ang, A.W. Mohammad, D. Johnson, N. Hilal, Unlocking the application potential of forward osmosis through integrated/hybrid process, Science of The Total Environment, (2019) 136047.

[58] Y. Cai, A critical review on draw solutes development for forward osmosis, Desalination, 391 (2016) 16-29.

[59] H. Luo, Q. Wang, T.C. Zhang, T. Tao, A. Zhou, L. Chen, X. Bie, A review on the recovery methods of draw solutes in forward osmosis, Journal of Water Process Engineering, 4 (2014) 212-223.

[60] L. Li, X.-p. Liu, H.-q. Li, A review of forward osmosis membrane fouling: types, research methods and future prospects, Environmental Technology Reviews, 6 (2017) 26-46.

[61] Q. She, R. Wang, A.G. Fane, C.Y. Tang, Membrane fouling in osmotically driven membrane processes: A review, Journal of Membrane Science, 499 (2016) 201-233.

[62] Y. Chun, D. Mulcahy, L. Zou, I.S. Kim, A short review of membrane fouling in forward osmosis processes, Membranes, 7 (2017) 30.

[63] C.S. Ong, P. Goh, W. Lau, N. Misdan, A.F. Ismail, Nanomaterials for biofouling and scaling mitigation of thin film composite membrane: A review, Desalination, 393 (2016) 2-15.

[64] W. Xu, Q. Ge, Synthetic polymer materials for forward osmosis (FO) membranes and FO applications: a review, Reviews in Chemical Engineering, 35 (2019) 191-209.

[65] W. Xu, Q. Chen, Q. Ge, Recent advances in forward osmosis (FO) membrane: Chemical modifications on membranes for FO processes, Desalination, 419 (2017) 101-116.

[66] Y.-N. Wang, K. Goh, X. Li, L. Setiawan, R. Wang, Membranes and processes for forward osmosis-based desalination: Recent advances and future prospects, Desalination, 434 (2018) 81-99.

[67] Q. Chen, W. Xu, Q. Ge, Progress in Forward Osmosis Membrane Separation Process, General Chemistry, 3 (2017).

[68] W.A. Suwaileh, D.J. Johnson, S. Sarp, N. Hilal, Advances in forward osmosis membranes: altering the sub-layer structure via recent fabrication and chemical modification approaches, Desalination, 436 (2018) 176-201. 
[69] M. Amini, M. Jahanshahi, A. Rahimpour, Synthesis of novel thin film nanocomposite (TFN) forward osmosis membranes using functionalized multi-walled carbon nanotubes, Journal of membrane science, 435 (2013) 233-241.

[70] L.A. Hoover, J.D. Schiffman, M. Elimelech, Nanofibers in thin-film composite membrane support layers: Enabling expanded application of forward and pressure retarded osmosis, Desalination, 308 (2013) 73-81.

[71] N.-N. Bui, M.L. Lind, E.M. Hoek, J.R. McCutcheon, Electrospun nanofiber supported thin film composite membranes for engineered osmosis, Journal of membrane science, 385 (2011) 10-19.

[72] F.E. Ahmed, B.S. Lalia, R. Hashaikeh, A review on electrospinning for membrane fabrication: challenges and applications, Desalination, 356 (2015) 15-30.

[73] Y. Zhang, T. Mu, M. Huang, G. Chen, T. Cai, H. Chen, L. Meng, X. Luo, Nanofiber composite forward osmosis (NCFO) membranes for enhanced antibiotics rejection: Fabrication, performance, mechanism, and simulation, Journal of Membrane Science, 595 (2020) 117425.

[74] W. Lau, A. Ismail, N. Misdan, M. Kassim, A recent progress in thin film composite membrane: a review, Desalination, 287 (2012) 190-199.

[75] J.R. McCutcheon, R.L. McGinnis, M. Elimelech, Desalination by ammonia-carbon dioxide forward osmosis: influence of draw and feed solution concentrations on process performance, Journal of membrane science, 278 (2006) 114-123.

[76] Z. Yang, H. Guo, C.Y. Tang, The upper bound of thin-film composite (TFC) polyamide membranes for desalination, Journal of Membrane Science, (2019) 117297.

[77] A. Soroush, J. Barzin, M. Barikani, M. Fathizadeh, Interfacially polymerized polyamide thin film composite membranes: Preparation, characterization and performance evaluation, Desalination, 287 (2012) 310-316.

[78] A. Tiraferri, N.Y. Yip, W.A. Phillip, J.D. Schiffman, M. Elimelech, Relating performance of thin-film composite forward osmosis membranes to support layer formation and structure, Journal of Membrane Science, 367 (2011) 340-352.

[79] G. Arthanareeswaran, V.M. Starov, Effect of solvents on performance of polyethersulfone ultrafiltration membranes: Investigation of metal ion separations, Desalination, 267 (2011) 57-63.

[80] S. Madaeni, A. Taheri, Preparation of PES ultrafiltration membrane for treatment of emulsified oily wastewater: Effect of solvent and non-solvent on morphology and performance, Journal of Polymer Engineering, 29 (2009) 183-198.

[81] N. Widjojo, T.-S. Chung, M. Weber, C. Maletzko, V. Warzelhan, The role of sulphonated polymer and macrovoid-free structure in the support layer for thin-film composite (TFC) forward osmosis (FO) membranes, Journal of membrane science, 383 (2011) 214-223.

[82] J. Jegal, S.G. Min, K.H. Lee, Factors affecting the interfacial polymerization of polyamide active layers for the formation of polyamide composite membranes, Journal of applied polymer science, 86 (2002) 2781-2787.

[83] C. Klaysom, S. Hermans, A. Gahlaut, S. Van Craenenbroeck, I.F. Vankelecom, Polyamide/Polyacrylonitrile (PA/PAN) thin film composite osmosis membranes: Film optimization, characterization and performance evaluation, Journal of membrane science, 445 (2013) 25-33.

[84] J. Wei, X. Liu, C. Qiu, R. Wang, C.Y. Tang, Influence of monomer concentrations on the performance of polyamide-based thin film composite forward osmosis membranes, Journal of membrane science, 381 (2011) 110-117.

44 [85] G. Han, S. Zhang, X. Li, N. Widjojo, T.-S. Chung, Thin film composite forward osmosis membranes 45 based on polydopamine modified polysulfone substrates with enhancements in both water flux and salt 46 rejection, Chemical Engineering Science, 80 (2012) 219-231. 
[86] W. Xie, G.M. Geise, B.D. Freeman, H.-S. Lee, G. Byun, J.E. McGrath, Polyamide interfacial composite membranes prepared from $\mathrm{m}$-phenylene diamine, trimesoyl chloride and a new disulfonated diamine, Journal of membrane science, 403 (2012) 152-161.

[87] G.-Y. Chai, W.B. Krantz, Formation and characterization of polyamide membranes via interfacial polymerization, Journal of Membrane Science, 93 (1994) 175-192.

[88] B. Khorshidi, T. Thundat, B. Fleck, M. Sadrzadeh, Thin film composite polyamide membranes: parametric study on the influence of synthesis conditions, RSC Advances, 5 (2015) 54985-54997.

[89] L. Huang, J.R. McCutcheon, Impact of support layer pore size on performance of thin film composite membranes for forward osmosis, Journal of Membrane Science, 483 (2015) 25-33.

[90] L. Huang, N.-N. Bui, M.T. Meyering, T.J. Hamlin, J.R. McCutcheon, Novel hydrophilic nylon 6, 6 microfiltration membrane supported thin film composite membranes for engineered osmosis, Journal of membrane science, 437 (2013) 141-149.

[91] P.S. Singh, S. Joshi, J. Trivedi, C. Devmurari, A.P. Rao, P. Ghosh, Probing the structural variations of thin film composite RO membranes obtained by coating polyamide over polysulfone membranes of different pore dimensions, Journal of membrane science, 278 (2006) 19-25.

[92] S.J. Kwon, S.-H. Park, M.G. Shin, M.S. Park, K. Park, S. Hong, H. Park, Y.-I. Park, J.-H. Lee, Fabrication of high performance and durable forward osmosis membranes using mussel-inspired polydopaminemodified polyethylene supports, Journal of Membrane Science, 584 (2019) 89-99.

[93] J. Wei, C. Qiu, C.Y. Tang, R. Wang, A.G. Fane, Synthesis and characterization of flat-sheet thin film composite forward osmosis membranes, Journal of Membrane Science, 372 (2011) 292-302.

[94] M. Tian, C. Qiu, Y. Liao, S. Chou, R. Wang, Preparation of polyamide thin film composite forward osmosis membranes using electrospun polyvinylidene fluoride (PVDF) nanofibers as substrates, Separation and Purification Technology, 118 (2013) 727-736.

[95] Z. Zhou, J.Y. Lee, T.-S. Chung, Thin film composite forward-osmosis membranes with enhanced internal osmotic pressure for internal concentration polarization reduction, Chemical Engineering Journal, 249 (2014) 236-245.

[96] Y. Sun, L. Xue, Y. Zhang, X. Zhao, Y. Huang, X. Du, High flux polyamide thin film composite forward osmosis membranes prepared from porous substrates made of polysulfone and polyethersulfone blends, Desalination, 336 (2014) 72-79.

[97] P. Hajighahremanzadeh, M. Abbaszadeh, S.A. Mousavi, M. Soltanieh, H. Bakhshi, Polyamide/polyacrylonitrile thin film composites as forward osmosis membranes, Journal of Applied Polymer Science, 133 (2016).

[98] L. Huang, J.T. Arena, J.R. McCutcheon, Surface modified PVDF nanofiber supported thin film composite membranes for forward osmosis, Journal of Membrane Science, 499 (2016) 352-360.

[99] Y. Zhao, X. Wang, Y. Ren, D. Pei, Mesh-embedded polysulfone (PSU)/sulfonated polysulfone (sPSU) supported thin film composite membranes for forward osmosis, (2017).

[100] S. Sahebi, S. Phuntsho, Y.C. Woo, M.J. Park, L.D. Tijing, S. Hong, H.K. Shon, Effect of sulphonated polyethersulfone substrate for thin film composite forward osmosis membrane, Desalination, 389 (2016) 129-136.

[101] M.J. Park, R.R. Gonzales, A. Abdel-Wahab, S. Phuntsho, H.K. Shon, Hydrophilic polyvinyl alcohol coating on hydrophobic electrospun nanofiber membrane for high performance thin film composite forward osmosis membrane, Desalination, 426 (2018) 50-59.

[102] S. Shokrollahzadeh, S. Tajik, Fabrication of thin film composite forward osmosis membrane using electrospun polysulfone/polyacrylonitrile blend nanofibers as porous substrate, Desalination, 425 (2018) 68-76.

[103] Q.-Y. Wu, X.-Y. Xing, Y. Yu, L. Gu, Z.-K. Xu, Novel thin film composite membranes supported by cellulose triacetate porous substrates for high-performance forward osmosis, Polymer, 153 (2018) 150160. 
[104] T. Ni, Q. Ge, Highly hydrophilic thin-film composition forward osmosis (FO) membranes functionalized with aniline sulfonate/bisulfonate for desalination, Journal of Membrane Science, 564 (2018) 732-741.

[105] M. Shibuya, M.J. Park, S. Lim, S. Phuntsho, H. Matsuyama, H.K. Shon, Novel CA/PVDF nanofiber supports strategically designed via coaxial electrospinning for high performance thin-film composite forward osmosis membranes for desalination, Desalination, 445 (2018) 63-74.

[106] K. Zheng, S. Zhou, X. Zhou, High-performance thin-film composite forward osmosis membrane fabricated on low-cost PVB/PVC substrate, New Journal of Chemistry, 42 (2018) 13382-13392.

[107] Y.-H. Chiao, A. Sengupta, S.-T. Chen, W.-S. Hung, J.-Y. Lai, L. Upadhyaya, X. Qian, S.R. Wickramasinghe, Novel thin-film composite forward osmosis membrane using polyethylenimine and its impact on membrane performance, Separation Science and Technology, (2019) 1-11.

[108] H.-E. Kwon, S.J. Kwon, S.-J. Park, M.G. Shin, S.-H. Park, M.S. Park, H. Park, J.-H. Lee, High performance polyacrylonitrile-supported forward osmosis membranes prepared via aromatic solvent-based interfacial polymerization, Separation and Purification Technology, 212 (2019) 449-457.

[109] M. Zheng, X. Zhao, S. Xu, D. Lu, Ultrathin Support-Free Membrane with High Water Flux for Forward Osmosis Desalination, Water, Air, \& Soil Pollution, 230 (2019) 138.

[110] K. Zheng, S. Zhou, Fabrication of a novel cyanoethyl cellulose substrate for thin-film composite forward osmosis membrane, Blue Green Systems, 1 (2019) 18-32.

[111] R. Thür, M. Corvilain, C. Klaysom, Y. Hartanto, I.F. Vankelecom, Tuning the selectivity of thin film composite forward osmosis membranes: effect of co-solvent and different interfacial polymerization synthesis routes, Separation and Purification Technology, (2019).

[112] M. Rastgar, A. Shakeri, H. Salehi, Study of polyamide thin film characteristics impact on permeability/selectivity performance and fouling behavior of forward osmosis membrane, Environmental Science and Pollution Research, 26 (2019) 1181-1191.

[113] W. Lee, P. Goh, W. Lau, C. Ong, A. Ismail, Antifouling zwitterion embedded forward osmosis thin film composite membrane for highly concentrated oily wastewater treatment, Separation and Purification Technology, 214 (2019) 40-50.

[114] X. Hao, S. Gao, J. Tian, Y. Sun, F. Cui, C.Y. Tang, Calcium-carboxyl intrabridging during interfacial polymerization: a novel strategy to improve antifouling performance of thin film composite membranes, Environmental science \& technology, 53 (2019) 4371-4379.

[115] J. Ma, T. Xiao, N. Long, X. Yang, The Role of Polyvinyl Butyral Additive in Forming Desirable Pore Structure for Thin Film Composite Forward Osmosis Membrane, Separation and Purification Technology, (2020) 116798.

[116] L. Shen, W.-s. Hung, J. Zuo, L. Tian, M. Yi, C. Ding, Y. Wang, Effect of ultrasonication parameters on forward osmosis performance of thin film composite polyamide membranes prepared with ultrasoundassisted interfacial polymerization, Journal of Membrane Science, (2020) 117834.

[117] C.F. Wan, T. Yang, G.G. Lipscomb, D.J. Stookey, T.-S. Chung, Design and fabrication of hollow fiber membrane modules, Journal of Membrane Science, 538 (2017) 96-107.

[118] S.-P. Sun, T.-S. Chung, Outer-selective pressure-retarded osmosis hollow fiber membranes from vacuum-assisted interfacial polymerization for osmotic power generation, Environmental science \& technology, 47 (2013) 13167-13174.

[119] R. Wang, L. Shi, C.Y. Tang, S. Chou, C. Qiu, A.G. Fane, Characterization of novel forward osmosis hollow fiber membranes, Journal of membrane science, 355 (2010) 158-167.

[120] P. Zhong, X. Fu, T.-S. Chung, M. Weber, C. Maletzko, Development of thin-film composite forward osmosis hollow fiber membranes using direct sulfonated polyphenylenesulfone (sPPSU) as membrane substrates, Environmental science \& technology, 47 (2013) 7430-7436.

[121] L. Luo, P. Wang, S. Zhang, G. Han, T.-S. Chung, Novel thin-film composite tri-bore hollow fiber membrane fabrication for forward osmosis, Journal of Membrane Science, 461 (2014) 28-38. 
[122] N. Peng, N. Widjojo, P. Sukitpaneenit, M.M. Teoh, G.G. Lipscomb, T.-S. Chung, J.-Y. Lai, Evolution of polymeric hollow fibers as sustainable technologies: Past, present, and future, Progress in Polymer Science, 37 (2012) 1401-1424.

[123] L. Shi, S. Chou, R. Wang, W. Fang, C. Tang, A. Fane, Effect of substrate structure on the performance of thin-film composite forward osmosis hollow fiber membranes, Journal of membrane science, 382 (2011) 116-123.

[124] P. Sukitpaneenit, T.-S. Chung, High performance thin-film composite forward osmosis hollow fiber membranes with macrovoid-free and highly porous structure for sustainable water production, Environmental science \& technology, 46 (2012) 7358-7365.

[125] S. Chou, L. Shi, R. Wang, C.Y. Tang, C. Qiu, A.G. Fane, Characteristics and potential applications of a novel forward osmosis hollow fiber membrane, Desalination, 261 (2010) 365-372.

[126] S. Lim, V.H. Tran, N. Akther, S. Phuntsho, H.K. Shon, Defect-free outer-selective hollow fiber thinfilm composite membranes for forward osmosis applications, Journal of Membrane Science, 586 (2019) 281-291.

[127] R.W. Baker, Membrane technology and applications. 2004, England: John Wiley and Sons, (2007). [128] C.Y. Tang, Q. She, W.C. Lay, R. Wang, A.G. Fane, Coupled effects of internal concentration polarization and fouling on flux behavior of forward osmosis membranes during humic acid filtration, Journal of membrane science, 354 (2010) 123-133.

[129] D.F. Sanders, Z.P. Smith, R. Guo, L.M. Robeson, J.E. McGrath, D.R. Paul, B.D. Freeman, Energyefficient polymeric gas separation membranes for a sustainable future: A review, Polymer, 54 (2013) 47294761.

[130] P. Wang, L. Luo, T.-S. Chung, Tri-bore ultra-filtration hollow fiber membranes with a novel triangleshape outer geometry, Journal of Membrane Science, 452 (2014) 212-218.

[131] J. Ren, J.R. McCutcheon, Polyacrylonitrile supported thin film composite hollow fiber membranes for forward osmosis, Desalination, 372 (2015) 67-74.

[132] M. Shibuya, M. Yasukawa, S. Mishima, Y. Tanaka, T. Takahashi, H. Matsuyama, A thin-film composite-hollow fiber forward osmosis membrane with a polyketone hollow fiber membrane as a support, Desalination, 402 (2017) 33-41.

[133] M. Yasukawa, S. Mishima, M. Shibuya, D. Saeki, T. Takahashi, T. Miyoshi, H. Matsuyama, Preparation of a forward osmosis membrane using a highly porous polyketone microfiltration membrane as a novel support, Journal of Membrane Science, 487 (2015) 51-59.

[134] Q. Jia, H. Han, L. Wang, B. Liu, H. Yang, J. Shen, Effects of CTAC micelles on the molecular structures and separation performance of thin-film composite (TFC) membranes in forward osmosis processes, Desalination, 340 (2014) 30-41.

[135] W. Lee, P.K. Kang, A.S. Kim, S. Lee, Impact of surface porosity on water flux and structural parameter in forward osmosis, Desalination, 439 (2018) 46-57.

[136] C.H. Tan, H.Y. Ng, Modified models to predict flux behavior in forward osmosis in consideration of external and internal concentration polarizations, Journal of Membrane science, 324 (2008) 209-219.

[137] C. Suh, S. Lee, Modeling reverse draw solute flux in forward osmosis with external concentration polarization in both sides of the draw and feed solution, Journal of membrane science, 427 (2013) 365374.

[138] N. Niksefat, M. Jahanshahi, A. Rahimpour, The effect of SiO 2 nanoparticles on morphology and performance of thin film composite membranes for forward osmosis application, Desalination, 343 (2014) 140-146.

[139] A.F. Faria, C. Liu, M. Xie, F. Perreault, L.D. Nghiem, J. Ma, M. Elimelech, Thin-film composite forward osmosis membranes functionalized with graphene oxide-silver nanocomposites for biofouling control, Journal of Membrane Science, 525 (2017) 146-156. 
[140] D. Emadzadeh, W. Lau, T. Matsuura, N. Hilal, A. Ismail, The potential of thin film nanocomposite membrane in reducing organic fouling in forward osmosis process, Desalination, 348 (2014) 82-88.

[141] A. Zirehpour, A. Rahimpour, A. Arabi Shamsabadi, M. Sharifian Gh, M. Soroush, Mitigation of thinfilm composite membrane biofouling via immobilizing nano-sized biocidal reservoirs in the membrane active layer, Environmental science \& technology, 51 (2017) 5511-5522.

[142] A. Rahimpour, S.F. Seyedpour, S. Aghapour Aktij, M. Dadashi Firouzjaei, A. Zirehpour, A. Arabi Shamsabadi, S. Khoshhal Salestan, M. Jabbari, M. Soroush, Simultaneous improvement of antimicrobial, antifouling, and transport properties of forward osmosis membranes with immobilized highly-compatible polyrhodanine nanoparticles, Environmental science \& technology, 52 (2018) 5246-5258.

[143] D. Emadzadeh, W.J. Lau, T. Matsuura, M. Rahbari-Sisakht, A. Ismail, A novel thin film composite forward osmosis membrane prepared from PSf-TiO 2 nanocomposite substrate for water desalination, Chemical Engineering Journal, 237 (2014) 70-80.

[144] D. Emadzadeh, W. Lau, M. Rahbari-Sisakht, H. Ilbeygi, D. Rana, T. Matsuura, A. Ismail, Synthesis, modification and optimization of titanate nanotubes-polyamide thin film nanocomposite (TFN) membrane for forward osmosis (FO) application, Chemical Engineering Journal, 281 (2015) 243-251.

[145] W. Lau, S. Gray, T. Matsuura, D. Emadzadeh, J.P. Chen, A. Ismail, A review on polyamide thin film nanocomposite (TFN) membranes: history, applications, challenges and approaches, water research, 80 (2015) 306-324.

[146] M. Rastgar, A. Shakeri, A. Bozorg, H. Salehi, V. Saadattalab, Impact of nanoparticles surface characteristics on pore structure and performance of forward osmosis membranes, Desalination, (2017). [147] M. Tian, Y.-N. Wang, R. Wang, A.G. Fane, Synthesis and characterization of thin film nanocomposite forward osmosis membranes supported by silica nanoparticle incorporated nanofibrous substrate, Desalination, 401 (2017) 142-150.

[148] N. Ma, J. Wei, R. Liao, C.Y. Tang, Zeolite-polyamide thin film nanocomposite membranes: towards enhanced performance for forward osmosis, Journal of membrane science, 405 (2012) 149-157.

[149] M. Rezaei-DashtArzhandi, M. Sarrafzadeh, P. Goh, W. Lau, A. Ismail, M. Mohamed, Development of novel thin film nanocomposite forward osmosis membranes containing halloysite/graphitic carbon nitride nanoparticles towards enhanced desalination performance, Desalination, 447 (2018) 18-28.

[150] A. Zirehpour, A. Rahimpour, M. Ulbricht, Nano-sized metal organic framework to improve the structural properties and desalination performance of thin film composite forward osmosis membrane, Journal of Membrane Science, 531 (2017) 59-67.

[151] A. Shakeri, H. Salehi, F. Ghorbani, M. Amini, H. Naslhajian, Polyoxometalate based thin film nanocomposite forward osmosis membrane: Superhydrophilic, anti-fouling, and high water permeable, Journal of colloid and interface science, 536 (2019) 328-338.

[152] A.L. Ohland, V.M.M. Salim, C.P. Borges, Nanocomposite membranes for osmotic processes: Incorporation of functionalized hydroxyapatite in porous substrate and in selective layer, Desalination, 463 (2019) 23-31.

[153] N. Ma, J. Wei, S. Qi, Y. Zhao, Y. Gao, C.Y. Tang, Nanocomposite substrates for controlling internal concentration polarization in forward osmosis membranes, Journal of membrane science, 441 (2013) 5462.

[154] M.J. Park, S. Phuntsho, T. He, G.M. Nisola, L.D. Tijing, X.-M. Li, G. Chen, W.-J. Chung, H.K. Shon, Graphene oxide incorporated polysulfone substrate for the fabrication of flat-sheet thin-film composite forward osmosis membranes, Journal of Membrane Science, 493 (2015) 496-507.

[155] X. Liu, H.Y. Ng, Fabrication of layered silica-polysulfone mixed matrix substrate membrane for enhancing performance of thin-film composite forward osmosis membrane, Journal of Membrane Science, 481 (2015) 148-163. 
[156] M. Tian, Y.-N. Wang, R. Wang, Synthesis and characterization of novel high-performance thin film nanocomposite (TFN) FO membranes with nanofibrous substrate reinforced by functionalized carbon nanotubes, Desalination, 370 (2015) 79-86.

[157] M. Ghanbari, D. Emadzadeh, W. Lau, H. Riazi, D. Almasi, A. Ismail, Minimizing structural parameter of thin film composite forward osmosis membranes using polysulfone/halloysite nanotubes as membrane substrates, Desalination, 377 (2016) 152-162.

[158] P. Lu, S. Liang, L. Qiu, Y. Gao, Q. Wang, Thin film nanocomposite forward osmosis membranes based on layered double hydroxide nanoparticles blended substrates, Journal of Membrane Science, 504 (2016) 196-205.

[159] P. Lu, S. Liang, T. Zhou, X. Mei, Y. Zhang, C. Zhang, A. Umar, Q. Wang, Layered double hydroxide/graphene oxide hybrid incorporated polysulfone substrate for thin-film nanocomposite forward osmosis membranes, RSC Advances, 6 (2016) 56599-56609.

[160] S. Lim, M.J. Park, S. Phuntsho, L.D. Tijing, G.M. Nisola, W.-G. Shim, W.-J. Chung, H.K. Shon, Duallayered nanocomposite substrate membrane based on polysulfone/graphene oxide for mitigating internal concentration polarization in forward osmosis, Polymer, 110 (2017) 36-48.

[161] Y.-H. Pan, Q.-Y. Zhao, L. Gu, Q.-Y. Wu, Thin film nanocomposite membranes based on imologite nanotubes blended substrates for forward osmosis desalination, Desalination, 421 (2017) 160-168.

[162] D. Ma, G. Han, S.B. Peh, S.B. Chen, Water-stable metal-organic framework UiO-66 for performance enhancement of forward osmosis membranes, Industrial \& Engineering Chemistry Research, 56 (2017) 12773-12782.

[163] L. Shen, S. Xiong, Y. Wang, Graphene oxide incorporated thin-film composite membranes for forward osmosis applications, Chemical Engineering Science, 143 (2016) 194-205.

[164] D. Emadzadeh, M. Ghanbari, W. Lau, M. Rahbari-Sisakht, T. Matsuura, A. Ismail, B. Kruczek, Solvothermal synthesis of nanoporous TiO2: the impact on thin-film composite membranes for engineered osmosis application, Nanotechnology, 27 (2016) 345702.

[165] D. Ma, S.B. Peh, G. Han, S.B. Chen, Thin-film nanocomposite (TFN) membranes incorporated with super-hydrophilic metal-organic framework (MOF) UiO-66: toward enhancement of water flux and salt rejection, ACS applied materials \& interfaces, 9 (2017) 7523-7534.

[166] S. Shokrgozar Eslah, S. Shokrollahzadeh, O. Moini Jazani, A. Samimi, Forward osmosis water desalination: Fabrication of graphene oxide-polyamide/polysulfone thin-film nanocomposite membrane with high water flux and low reverse salt diffusion, Separation Science and Technology, 53 (2018) 573583.

[167] S.F. Seyedpour, A. Rahimpour, A.A. Shamsabadi, M. Soroush, Improved performance and antifouling properties of thin-film composite polyamide membranes modified with nano-sized bactericidal graphene quantum dots for forward osmosis, Chemical Engineering Research and Design, 139 (2018) 321-334.

[168] M.G.N. Perera, Y.R. Galagedara, Y. Ren, M. Jayaweera, Y. Zhao, R. Weerasooriya, Fabrication of fullerenol-incorporated thin-film nanocomposite forward osmosis membranes for improved desalination performances, Journal of Polymer Research, 25 (2018) 199.

[169] R.R. Darabi, M. Jahanshahi, M. Peyravi, A support assisted by photocatalytic Fe304/ZnO nanocomposite for thin-film forward osmosis membrane, Chemical Engineering Research and Design, 133 (2018) 11-25.

[170] R.R. Darabi, M. Peyravi, M. Jahanshahi, Modified forward osmosis membranes by two aminofunctionalized ZnO nanoparticles: A comparative study, Chemical Engineering Research and Design, 145 (2019) 85-98.

[171] S.-J. Shi, Y.-H. Pan, S.-F. Wang, Z.-W. Dai, L. Gu, Q.-Y. Wu, Aluminosilicate Nanotubes Embedded Polyamide Thin Film Nanocomposite Forward Osmosis Membranes with Simultaneous Enhancement of Water Permeability and Selectivity, Polymers, 11 (2019) 879. 
[172] A. Shakeri, H. Mighani, N. Salari, H. Salehi, Surface modification of forward osmosis membrane using polyoxometalate based open frameworks for hydrophilicity and water flux improvement, Journal of Water Process Engineering, 29 (2019) 100762.

[173] N. Akther, S. Lim, V.H. Tran, S. Phuntsho, Y. Yang, T.-H. Bae, N. Ghaffour, H.K. Shon, The effect of Schiff base network on the separation performance of thin film nanocomposite forward osmosis membranes, Separation and Purification Technology, 217 (2019) 284-293.

[174] R. Dai, X. Zhang, M. Liu, Z. Wu, Z. Wang, Porous metal organic framework CuBDC nanosheet incorporated thin-film nanocomposite membrane for high-performance forward osmosis, Journal of membrane science, 573 (2019) 46-54.

[175] A. Shakeri, H. Salehi, S.R. Razavi, S.M.M. Babaheydari, Blue lemon@ quaternary graphene oxide open frameworks: As a novel nanostructure for performance enhancement of thin film nanocomposite forward osmosis membrane, Chemical Engineering Research and Design, 148 (2019) 451-459.

[176] A. Shakeri, R. Razavi, H. Salehi, M. Fallahi, T. Eghbalazar, Thin film nanocomposite forward osmosis membrane embedded with amine-functionalized ordered mesoporous silica, Applied Surface Science, 481 (2019) 811-818.

[177] M. Shan, H. Kang, Z. Xu, N. Li, M. Jing, Y. Hu, K. Teng, X. Qian, J. Shi, L. Liu, Decreased cross-linking in interfacial polymerization and heteromorphic support between nanoparticles: Towards high-water and low-solute flux of hybrid forward osmosis membrane, Journal of colloid and interface science, 548 (2019) 170-183.

[178] M. Bagherzadeh, A. Bayrami, M. Amini, Enhancing forward osmosis (FO) performance of polyethersulfone/polyamide (PES/PA) thin-film composite membrane via the incorporation of GQDs@ UiO-66-NH2 particles, Journal of Water Process Engineering, 33 (2020) 101107.

[179] W. Ding, Y. Li, M. Bao, J. Zhang, C. Zhang, J. Lu, Highly permeable and stable forward osmosis (FO) membrane based on the incorporation of $\mathrm{Al} 2 \mathrm{O} 3$ nanoparticles into both substrate and polyamide active layer, RSC Advances, 7 (2017) 40311-40320.

[180] W. Fang, R. Wang, S. Chou, L. Setiawan, A.G. Fane, Composite forward osmosis hollow fiber membranes: Integration of RO-and NF-like selective layers to enhance membrane properties of antiscaling and anti-internal concentration polarization, Journal of membrane science, 394 (2012) 140-150. [181] C.Y. Tang, Q. She, W.C. Lay, R. Wang, R. Field, A.G. Fane, Modeling double-skinned FO membranes, Desalination, 283 (2011) 178-186.

[182] K.Y. Wang, R.C. Ong, T.-S. Chung, Double-skinned forward osmosis membranes for reducing internal concentration polarization within the porous sublayer, Industrial \& Engineering Chemistry Research, 49 (2010) 4824-4831.

[183] S. Zhang, K.Y. Wang, T.-S. Chung, H. Chen, Y. Jean, G. Amy, Well-constructed cellulose acetate membranes for forward osmosis: minimized internal concentration polarization with an ultra-thin selective layer, Journal of Membrane Science, 360 (2010) 522-535.

[184] G. Han, Z.L. Cheng, T.-S. Chung, Thin-film composite (TFC) hollow fiber membrane with doublepolyamide active layers for internal concentration polarization and fouling mitigation in osmotic processes, Journal of Membrane Science, 523 (2017) 497-504.

[185] L. Li, M. Chen, Y. Dong, X. Dong, S. Cerneaux, S. Hampshire, J. Cao, L. Zhu, Z. Zhu, J. Liu, A low-cost alumina-mullite composite hollow fiber ceramic membrane fabricated via phase-inversion and sintering method, Journal of the European Ceramic Society, 36 (2016) 2057-2066.

[186] P.H. Duong, T.-S. Chung, S. Wei, L. Irish, Highly permeable double-skinned forward osmosis membranes for anti-fouling in the emulsified oil-water separation process, Environmental science \& technology, 48 (2014) 4537-4545.

[187] X. Song, L. Wang, C.Y. Tang, Z. Wang, C. Gao, Fabrication of carbon nanotubes incorporated doubleskinned thin film nanocomposite membranes for enhanced separation performance and antifouling capability in forward osmosis process, Desalination, 369 (2015) 1-9. 
[188] C.S. Ong, B. Al-anzi, W.J. Lau, P.S. Goh, G.S. Lai, A.F. Ismail, Y.S. Ong, Anti-Fouling Double-Skinned Forward Osmosis Membrane with Zwitterionic Brush for Oily Wastewater Treatment, Scientific reports, 7 (2017) 6904.

[189] X. Zhang, M. Xie, Z. Yang, H.-C. Wu, C. Fang, L. Bai, L.-F. Fang, T. Yoshioka, H. Matsuyama, Antifouling Double-Skinned Forward Osmosis Membranes by Constructing Zwitterionic Brush-Decorated MWCNT Ultrathin Films, ACS applied materials \& interfaces, (2019).

[190] A.M. Awad, R. Jalab, J. Minier-Matar, S. Adham, M.S. Nasser, S. Judd, The status of forward osmosis technology implementation, Desalination, 461 (2019) 10-21.

[191] J. Ren, J.R. McCutcheon, A new commercial thin film composite membrane for forward osmosis, Desalination, 343 (2014) 187-193.

[192] J. Ren, J.R. McCutcheon, A new commercial biomimetic hollow fiber membrane for forward osmosis, Desalination, 442 (2018) 44-50.

[193] M. Shibuya, M. Yasukawa, T. Takahashi, T. Miyoshi, M. Higa, H. Matsuyama, Effect of operating conditions on osmotic-driven membrane performances of cellulose triacetate forward osmosis hollow fiber membrane, Desalination, 362 (2015) 34-42.

[194] A. Ebadi Amooghin, S. Mashhadikhan, H. Sanaeepur, A. Moghadassi, T. Matsuura, S. Ramakrishna, Substantial breakthroughs on function-led design of advanced materials used in mixed matrix membranes 18 (MMMs): A new horizon for efficient CO2 separation, PROGRESS IN MATERIALS SCIENCE, 102 (2019) 22219295.

20

21

\section{ROMAN LETTERS}

$\begin{array}{ll}A & \text { water permeability coefficient }(\mathrm{LMH} / \mathrm{bar}, \mathrm{m} / \mathrm{s} \mathrm{pa}) \\ B & \text { solute permeability coefficient }(\mathrm{LMH}, \mathrm{m} / \mathrm{s}) \\ C & \text { Solute concentration }(\mathrm{mol} / \mathrm{L}) \\ J_{S} & \text { Reverse salt flux }(\mathrm{gMH}) \\ J_{S} / J_{v} & \text { specific reverse salt flux }(\mathrm{g} / \mathrm{L}) \\ J_{v} & \text { water flux }(\mathrm{LMH}) \\ l & \text { Thickness of the substrate layer }(\mu \mathrm{m}) \\ n & \text { The van't Hoff coefficient } \\ P & \text { Hydraulic pressure } \\ R & \text { Rejection rate or Universal gas constant } \\ S & \text { Structural parameter }(\mathrm{m}, \mathrm{mm}, \mu \mathrm{m})\end{array}$

22

\section{GREEK LETTERS}

$\varepsilon \quad$ Porosity of the substrate layer

$\pi \quad$ Osmotic pressure (bar)

$\tau \quad$ Tortuosity 


\section{SUBSCRIPTS}

$\begin{array}{ll}\text { draw } & \text { Draw active layer } \\ \text { feed } & \text { Feed active layer } \\ s / f & \text { the interface between the substrate and the feed active layer } \\ \text { support } & \text { substrate layer }\end{array}$

ABBREVIATIONS

AAPTS

AL-DS

AL-FS

ANTs

CA

CAB

CEC

CFIC

CMPs

CNTs

COFs

CTA

CTAC

CTFs

DI water

DPE

DSA-2Na

ECP

FO

g-C3N4

gMH

$\mathrm{gMH} / \mathrm{bar}$

$\mathrm{GO}$

GQDs

Hapf

$\mathrm{HF}$

HNTs

HTI

ICIC

ICP

INTs

IP

IPA
N-(2-Aminoethyl)-3-aminopropyltrimethoxysilane

Active layer facing the draw solution mode; also called as PRO mode Active layer facing the feed solution mode; also called as FO mode aluminosilicate nanotubes

cellulose acetate

Cellulose acetate butyrate

Cyanoethyl Cellulose

5-chloroformyloxy-isophthaloylchloride

Conjugated microporous polymers

carbon nanotubes

Covalent organic frameworks

Cellulose triacetate

Cetyltrimethylammonium chloride

Covalent triazine based frameworks

Deionized water

Polydopamine-modified polyethylene

2,5-disulfoaniline disodium salt

External concentration polarization

Forward osmosis

Graphite-like carbon nitrite

Grams per square meter per hour

Grams per square meter per hour per bar

Graphene oxide

graphene quantum dots

hydroxyapatite particles

Hollow fiber

Halloysite nanotubes HNTs

Hydration Technologies Inc

5-isocyanato-isophthaloyl chloride

Internal concentration polarization

Imogolite nanotubes

Interfacial polymerization

Isopropyl alcohol 


\begin{tabular}{|c|c|}
\hline IPC & Isophthaloyl chloride \\
\hline LDH & Layered double hydroxide \\
\hline $\mathrm{LiCl}$ & Lithium chloride \\
\hline LMH & Liters per square meter per hour \\
\hline $\mathrm{LMH} / \mathrm{bar}$ & Liters per square meter per hour per bar \\
\hline MOF & Metal-organic framework \\
\hline MOPs & Metal organic polyhedras \\
\hline MPD & M-Phenylenediamine \\
\hline MWCNT/PSBMA & Zwitterionic brush-decorated, multiwalled carbon nanotube \\
\hline $\mathrm{NF}$ & Nanofiltration \\
\hline $\mathrm{NH}_{2}$ - TNTs & Amino-functionalized titanate nanotubes \\
\hline NMP & N-Methyl-2-pyrrolidone \\
\hline OCNTs & Oxidized carbon nanotubes \\
\hline PA & Polyamide \\
\hline PAFs & Porous aromatic frameworks \\
\hline PAI & Poly (amide-imide) \\
\hline PAN & Polyacrylonitrile \\
\hline PBI & Polybenzimidazole \\
\hline PCPs & Porous coordination polymers \\
\hline PDA & Polydopamine \\
\hline PE & Polyethylene \\
\hline$P E G$ & Polyethylene glycol \\
\hline PEI & Polyetherimide or Polyethyleneimine \\
\hline PES & Polyethersulfone \\
\hline PIP & Piperazine \\
\hline POF & Porous organic frameworks \\
\hline POM-OFs & Polyoxometalate-based open frameworks \\
\hline PPD & P-phenylene diamine \\
\hline PSF & Polysulfone \\
\hline PVA & Polyvinyl alcohol \\
\hline PVB & Poly (vinyl butyral) \\
\hline PVC & Poly (vinyl chloride) \\
\hline PVDF & Polyvinylidene fluoride \\
\hline RO & Reverse osmosis \\
\hline SBA-15-NH2 & mesoporous silica modified with amine \\
\hline SNW-1 & Schiff base network-1 \\
\hline sPEEK & Sulfonated polyetheretherketone \\
\hline SPPO & Sulfonated poly (phenylene oxide) \\
\hline sPPSU & Sulfonated poly phenylene sulfone \\
\hline sPSF & Sulfonated polysulfone \\
\hline
\end{tabular}


SWCNTs

SWo

TEOA

TFC

TFC-A

TFC-D

TFC-N

TMC

TNTs

UiO-66

ZIF

ZLD

ZNPs

ZSCSNPs
Single wall carbon nanotubes

Spiral wound

Triethanolamine

Thin film composite

TFC with polyamide active layer

Double-skinned TFC

Thin film nanocomposites

Trimesoyl Chloride

Titanate nanotubes

zirconium (IV)-carboxylate metal-organic framework

Zeolitic imidazolate framework

Zero liquid discharge

Zinc oxide nanoparticles

$\mathrm{ZnO}-\mathrm{SiO} 2$ core-shell nanoparticles 


\section{Declaration of interests}

૫ The authors declare that they have no known competing financial interests or personal relationships that could have appeared to influence the work reported in this paper.

$\square$ The authors declare the following financial interests/personal relationships which may be considered as potential competing interests:

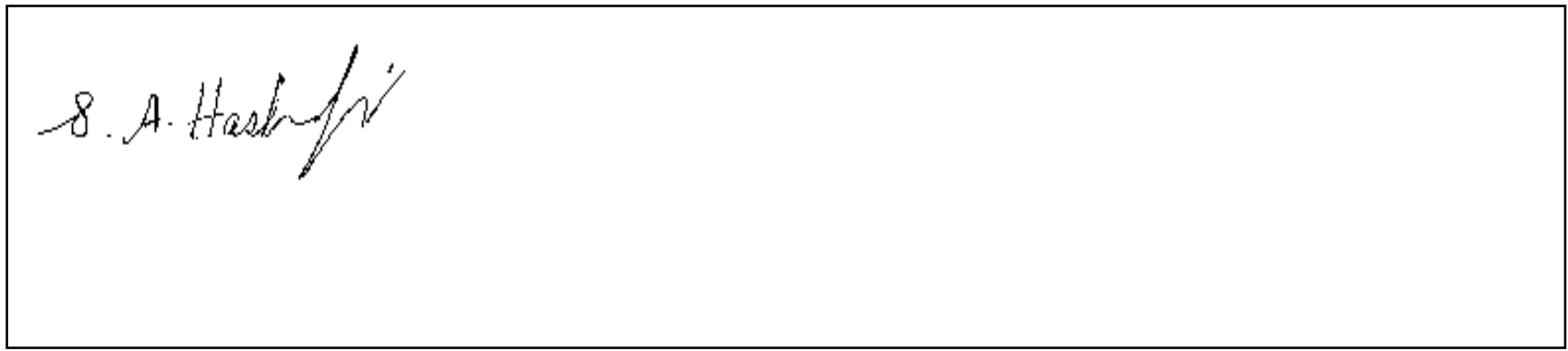

